FinAL SUMmARY REPORT

FOR

\title{
THE MISSISSIPPI UNIVERSITY RESEARCH CONSORTIUM FOR THE UTILIZATION OF BIOMASS: PRODUCTION OF ALTERNATIVE FUELS FROM WASTE BIOMASS INITIATIVE
}

Submitted to

EPSCoR Office US DOE Division of Basic Science

Participating Universities

Mississippi State University

Jackson State University

University of Mississippi

University of Southern Mississippi

March 2009 


\section{EXECUTIVE SUMMARY}

The Mississippi University Research Consortium for the Utilization of Biomass

The Mississippi Consortium for the Utilization of Biomass was formed via funding from the US Department of Energy's EPSCoR Program, which is administered by the Office of Basic Science. Funding was approved in July of 1999 and received by participating Mississippi institutions by 2000. The project was funded via two 3-year phases of operation (the second phase was awarded based on the high merits observed from the first 3-year phase), with funding ending in 2007. The mission of the Consortium was to promote the utilization of biomass, both cultured and waste derived, for the production of commodity and specialty chemicals. These scientific efforts, although generally basic in nature, are key to the development of future industries within the Southeastern United States. In this proposal, the majority of the efforts performed under the DOE EPSCOR funding were focused primarily toward the production of ethanol from lignocellulosic feedstocks and biogas from waste products. However, some of the individual projects within this program investigated the production of other products from biomass feeds (i.e. acetic acid and biogas) along with materials to facilitate the more efficient production of chemicals from biomass.

Mississippi is a leading state in terms of raw biomass production. Its top industries are timber, poultry production, and row crop agriculture. However, for all of its vast amounts of biomass produced on an annual basis, only a small percentage of the biomass is actually industrially produced into products, with the bulk of the biomass being wasted. This situation is actually quite representative of many Southeastern US states. The research and development efforts performed attempted to further develop promising chemical production techniques that use Mississippi biomass feedstocks. The three processes that were the primary areas of interest for ethanol production were syngas fermentation, acid hydrolysis followed by hydrolyzate fermentation, and enzymatic conversion. All three of these processes are of particular interest to states in the Southeastern US since the agricultural products produced in this region are highly variable in terms of actual crop, production quantity, and the ability of land areas to support a particular type of crop. This greatly differs from the Midwestern US where most of this region's agricultural land supports one to two primary crops, such as corn 
and soybean. Therefore, developing processes which are relatively flexible in terms of biomass feedstock is key to the southeastern region of the US if this area is going to be a "player" in the developing biomass to chemicals arena. With regard to the fermentation of syngas, research was directed toward developing improved biocatalysts through organism discovery and optimization, improving ethanol/acetic acid separations, evaluating potential bacterial contaminants, and assessing the use of innovative fermentors that are better suited for supporting syngas fermentation. Acid hydrolysis research was directed toward improved conversion yields and rates, acid recovery using membranes, optimization of fermenting organisms, and hydrolyzate characterization with changing feedstocks. Additionally, a series of development efforts addressed novel separation techniques for the separation of key chemicals from fermentation activities.

Biogas related research focused on key factors hindering the widespread use of digester technologies in non-traditional industries. The digestion of acetic acids and other fermentation wastewaters was studied and methods used to optimize the process were undertaken. Additionally, novel laboratory methods were designed along with improved methods of digester operation. A search for better performing digester consortia was initiated coupled with improved methods to initiate their activity within digester environments.

The third activity of the consortium generally studied the production of "other" chemicals from waste biomass materials found in Mississippi. The two primary examples of this activity are production of chemical feedstocks from lignin and the production of high valued lipids from wastewater treatment sludges. Lignin conversion research, done in collaboration with DOE's National Renewable Energy Laboratory (NREL), studied ligninases derived from bacteria found within the gut of wood degrading insects, such as termites and the Betsey beetle. This research attempted to use these enzymes to reduce lignin down to aromatic chemicals capable of chemical conversation for production of value-added chemicals. The biodiesel efforts attempted to development economically viable methods for the separation of lipids from wastewater bacteria (which make up the bulk of sewage sludge) which were then converted to biodisel. 
The development of increased capability to obtain competitive research funding, sustain economic development within Mississippi for attracting alternative energy companies, the partnering with national leaders in the biofuels arena, and the technology transfer of produced new knowledge was also a key focus of the Consortium. Over 30 peer-reviewed papers have been published or will soon be published as a result of the Consortium's activities. Well over 150 technical presentations have been made to date by Consortium members as a means of rapid technology transfer. Collaboration with over 20 entities from national laboratories, other institutions, and non-profits developed over the six-year funding period. Student involvement included more than 100 students (graduate and undergraduate) with over $30 \%$ being from under-represented groups within the science and technology fields (females and minorities). Finally, a ratio of return from the original DOE EPSCoR funds to external "new" funds of over 4:1 was achieved, indicating a high level of success for positioning Mississippi universities to assist with the development of biofuels and renewable chemicals on a national platform. Numerous companies and government entities provided technical assistance with optimizing existing production facilities and/or the design of new processes within Mississippi.

In summary, the EPSCoR funded project literally has transformed the State of Mississippi from essentially a non-player in the biofuels and renewable chemicals arena into a leader within the US; particularly in the processing aspect of this industry. From the perspective of the State of Mississippi, it is strongly believed that the EPSCoR funding was a key initiating point which resulted in a dramatic improvement in the ability of Mississippi universities to compete both at the national level and internationally as observed by the recent initiation of international collaborations. Finally, even though the funding via EPSCoR has ended, a high level of collaboration and interaction remains among the Consortium members. The EPSCoR project results have greatly enhanced the capabilities of the State Mississippi in targeted areas. 


\section{PREFACE}

This report summarizes the activities of the EPSCoR project funded by the US Department of Energy (DOE) awarded to a four university group in the State of Mississippi. These universities united under a research consortium entitled "The Mississippi University Research Consortium for the Utilization of Biomass" to formulate the project team. The member universities were Mississippi State University (the lead entity), Jackson State University (a historically black institution), the University of Mississippi, and the University of Southern Mississippi. Over 15 faculty served as coprincipal investigators on this project, with Dr. Mark E. Zappi, P.E. serving as the director and principal investigator from its inception to 2005 (in 2005, Dr. Zappi left Mississippi State University to assume the deanship at the University of Louisiana's College of Engineering). In Fall 2005, Dr. Todd French of Mississippi State University took over the lead of the project while working in concert with Dr. Zappi who remained as Director as a means of smoothly completing the grant.

The authors of this report were the involved faculty from all four member universities. Drs. Zappi and French served as chief editors to organize the many volumes of reported data and conclusions provided to them by the team. It is important to recognize the lead faculty CO-PIs from each member institution who were directly involved over the full course of this effort. They are, from Mississippi State University: Drs. Mark Zappi, Todd French, Lewis Brown, Clifford George, Rafael Henandez, and Marvin Salin; from Jackson State University: Drs. Huey-Min Hwang, Ken Lee, Yi Zhang, and Maria Begonia; from the University of Mississippi: Drs. Clint Williford and Al Mikell; from the University of Southern Mississippi: Drs. Robert Moore and Roger Hester. Additionally, the project team would like to recognize the efforts of the many graduate students whose project work resulted in much of the research effort detailed in this report. Their contribution to this report is gratefully acknowledged.

The primary DOE project manager for this project was Dr. Matesh Varma, who retired from DOE in 2005. The project team would like to extend their deepest appreciate to Dr. Varma for all of his guidance and support over the course of the project. 
In total, $\$ 3.6$ million was provided to the Consortium by the DOE EPSCoR Office. The member universities matched this funding to yield a total project worth of $\$ 7.2$ million over a seven year period of operations. The project team extends their sincerest appreciation to both the US DOE and the administrative entities from each university for the support.

This report is drafted to provide a summary of the many initiatives - both research and program development - that the Consortium undertook over the course of the funding period. It is divided into three sections:

SECTION I - INTRODUCTION - provides the framework of the project development strategy used to design the project along with details on how the State of Mississippi was positioned in terms of supporting bio-based energy development prior to the initiation of the project.

SECTION II - CAPABILITY DEVELOPMENT - summarizes gains in the capability of both the project team and the State of Mississippi to be key players within the bio-based energy arena.

SECTION III - SUMMARY OF RESEARCH ACTIVITIES - provides a summary of each individual project performed under this EPSCoR funded effort. More detail on various research initiatives along with rationale for selecting these topics are presented in the body of this report. 


\section{TABLE OF CONTENTS}

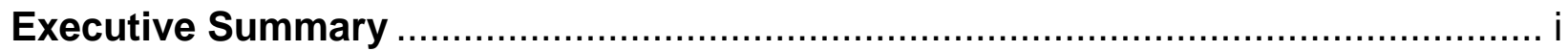

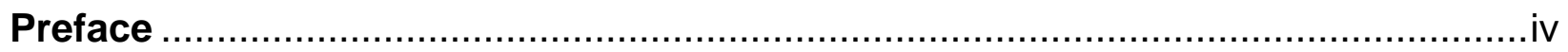

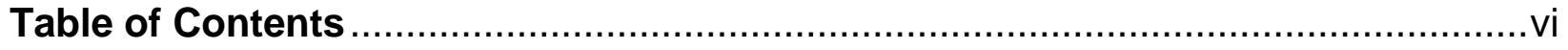

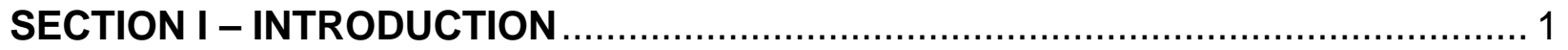

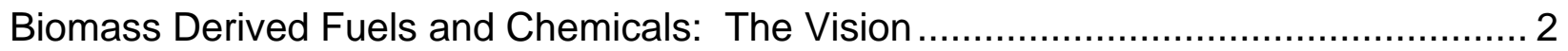

Potential for Mississippi to Become a Leader in

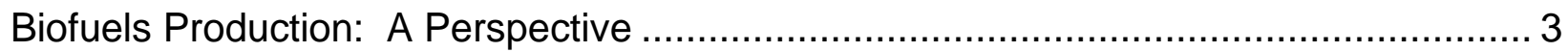

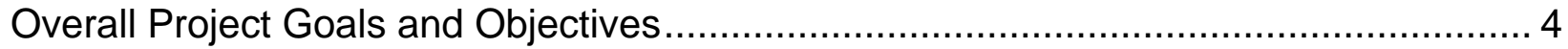

Overview of Research and Development Efforts:

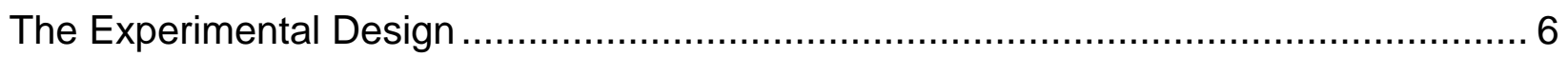

Key Research Topics Evaluated During the Project....................................... 6

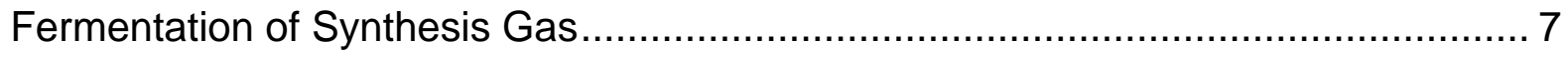

Ethanol Production Via the Fermentation of Acid Hydrolyzate........................... 8

Biogas Production from Wastewaters ................................................... 10

Development of Novel Polymers for use in

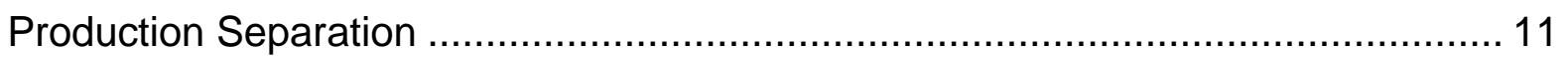

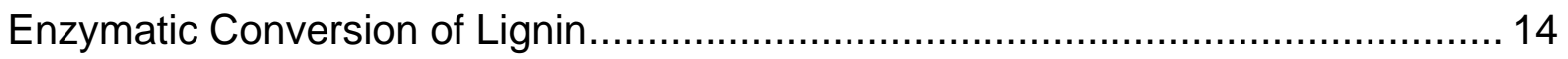

Production of Biodiesel from a Novel Feedstock ...................................... 16

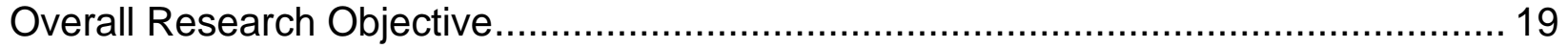

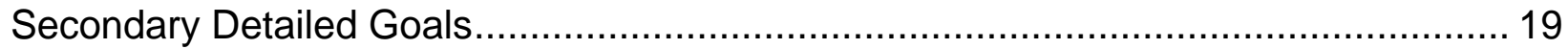

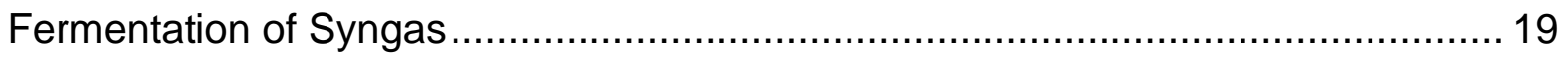

Acid Hydrolysis of Biomass to Produce Ethanol ...................................... 20

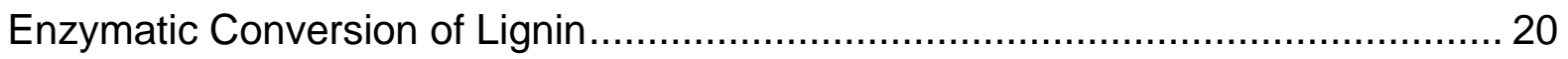

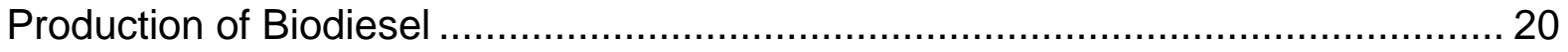

SECTION II - CAPABILITY DEVELOPMENT WITHIN THE STATE OF

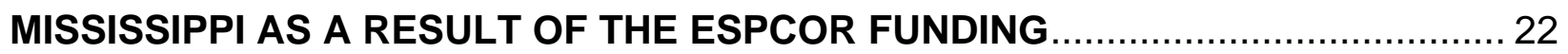

Development of Mississippi as a Leader in the Bio-Based

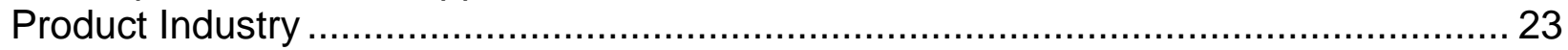

Research Competitiveness and Capability Development................................... 23

Peer-Reviewed Papers Published Via Work Derived from the Program .................... 24 


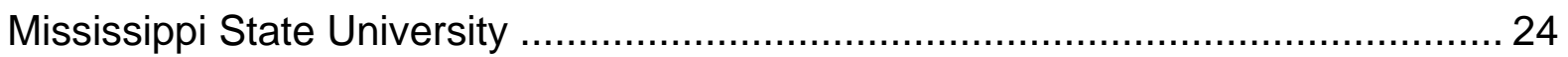

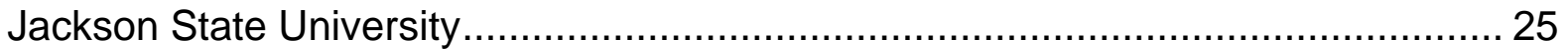

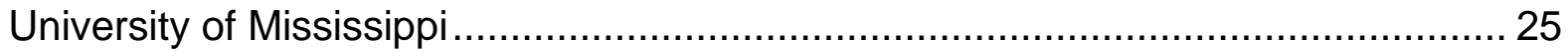

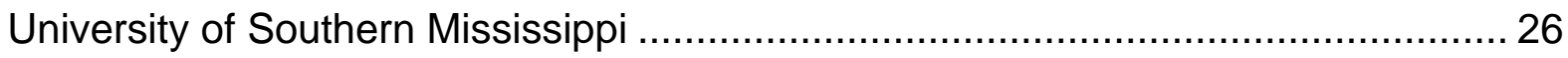

Projects Obtained as a Result of Capability Enhancement ........................................ 26

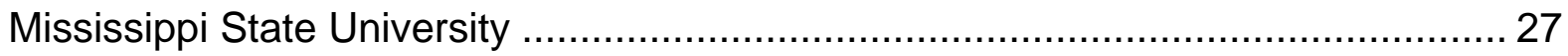

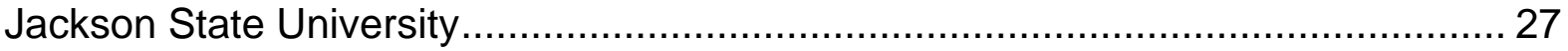

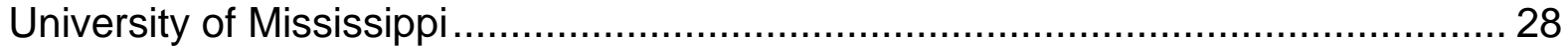

Other Research Competiveness and Capability Improvements ................................ 28

Faculty Active in Bio-Based Product Development

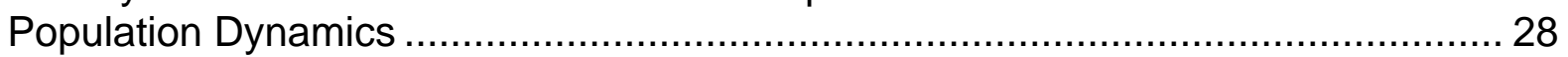

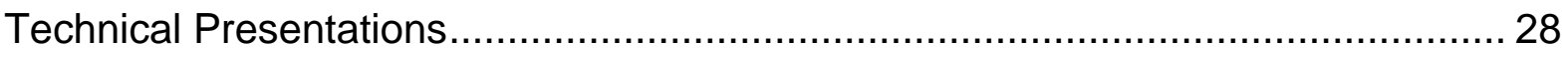

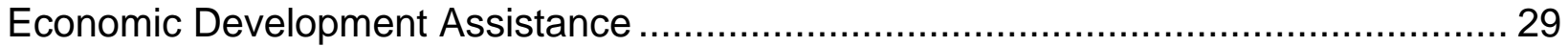

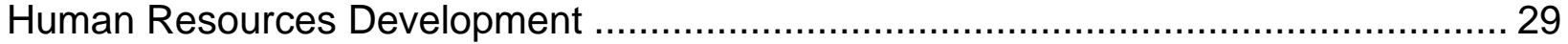

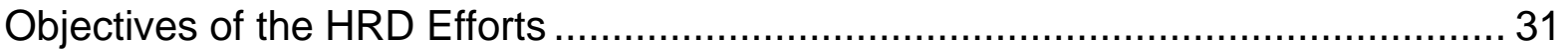

Overview of the Consortium HRD Activities ....................................................... 32

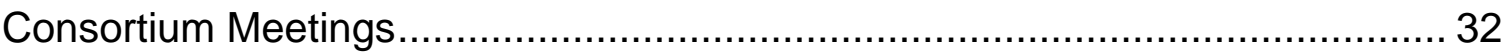

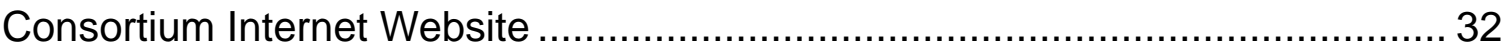

DOE and Other Federal Facility Visits ..................................................... 33

Consortium Researcher/Student/Equipment Exchange ................................. 33

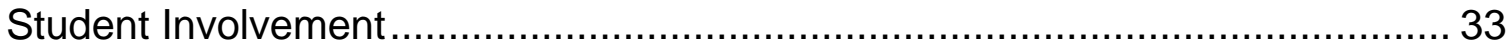

Development of Relationships with Key Entities ................................................. 37

SECTION III - SUMMARY OF RESEARCH ACTIVITIES …................................ 38

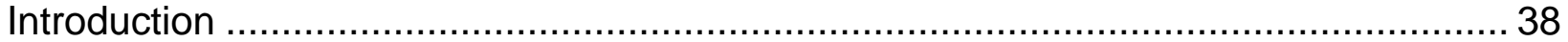

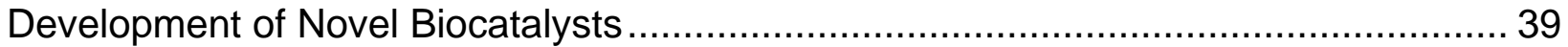

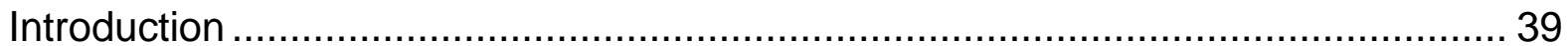

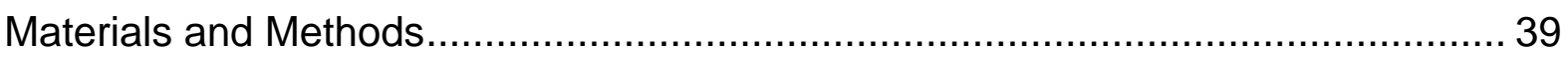

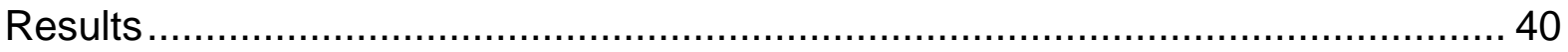

Work with ATCC Cultures..................................................................... 40

Isolation of Mesophilic Carbon Monoxide-Utilizing Cultures .............................. 40

Evaluation of Immobilized Cell Technology .................................................. 45

Cultures that Produce Ethanol from Carbon Monoxide at $90^{\circ} \mathrm{C}$....................... 45 


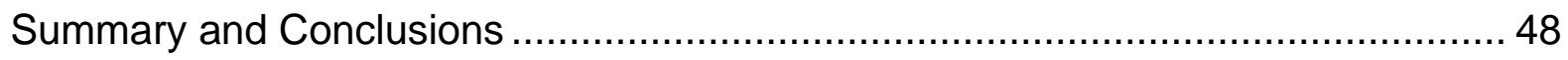

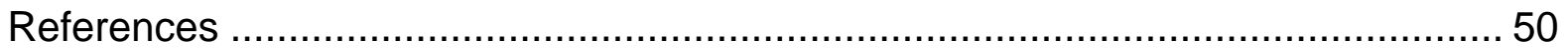

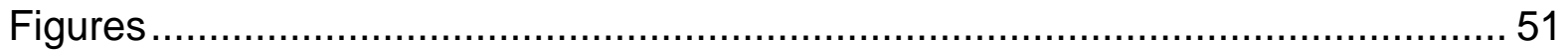

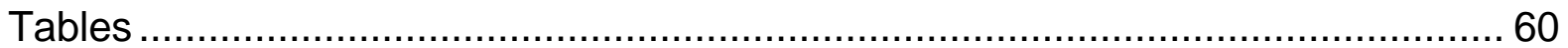

Development and Comparisons of Efficient Gas-Cultivation Systems

For Anaerobic Carbon Monoxide Utilizing Organisms .............................................6 66

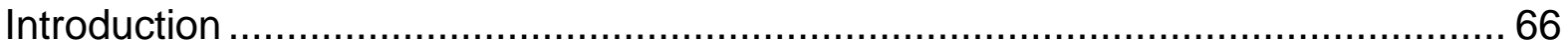

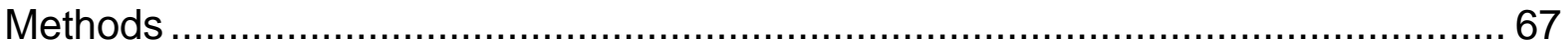

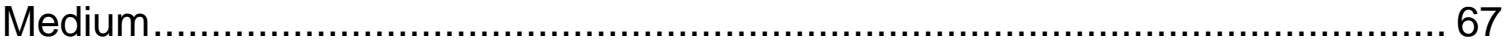

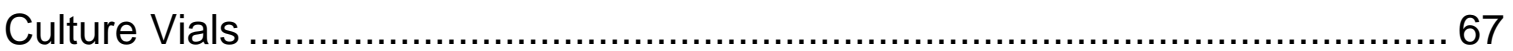

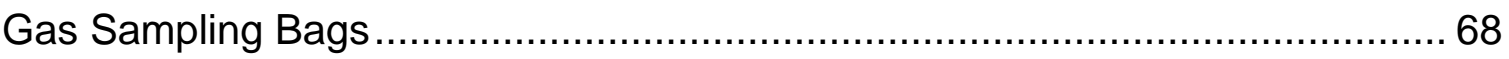

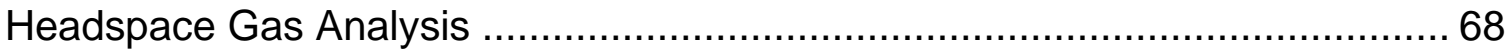

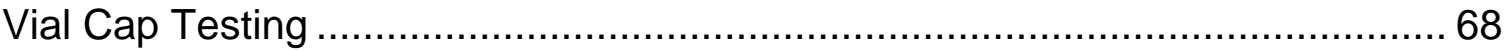

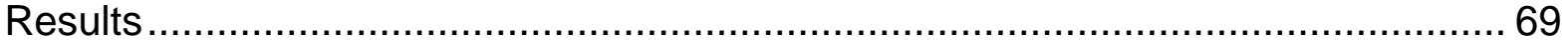

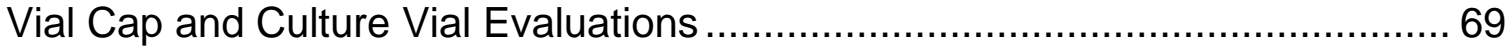

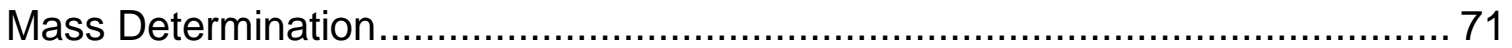

Cultivation of CO Utilizing Microorganism ................................................ 72

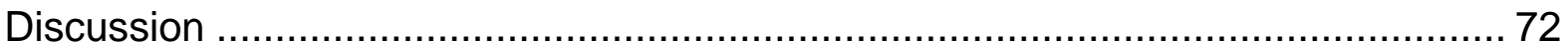

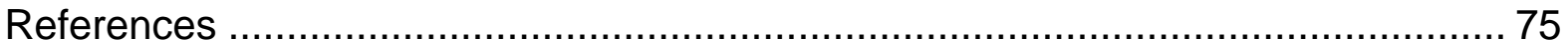

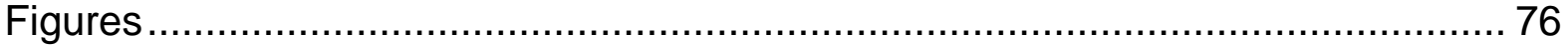

Evaluation of the Impact of Nutrient Solution Composition and Nutrient Solution Dosing on the Methanogenic Conversion of a

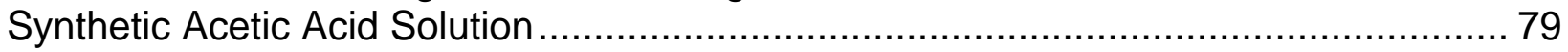

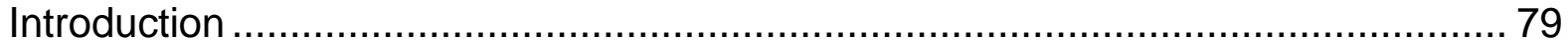

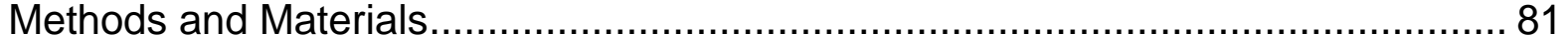

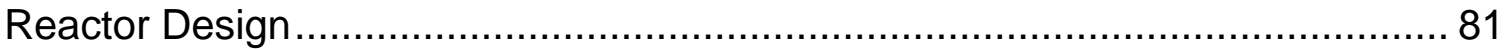

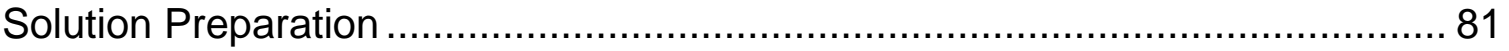

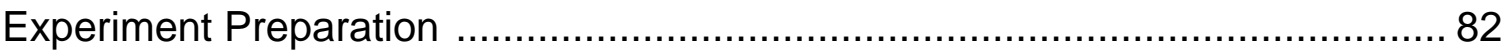

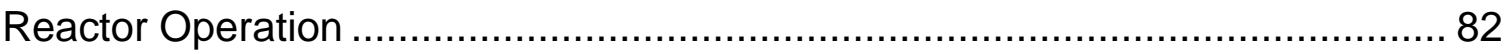

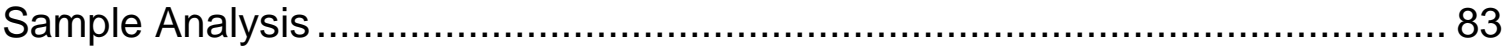

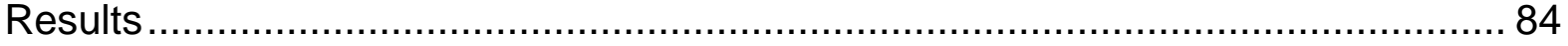

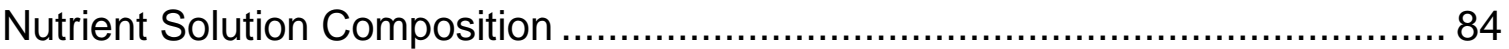

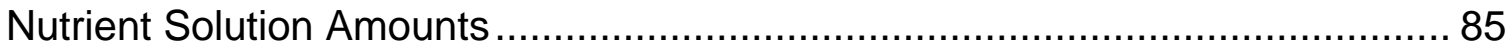




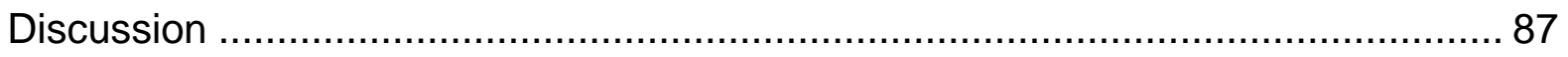

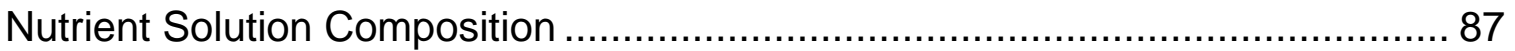

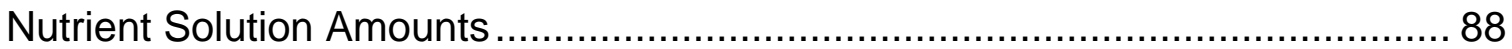

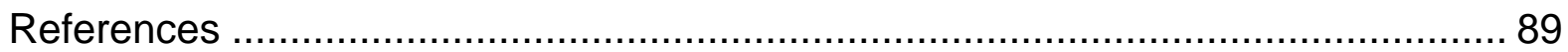

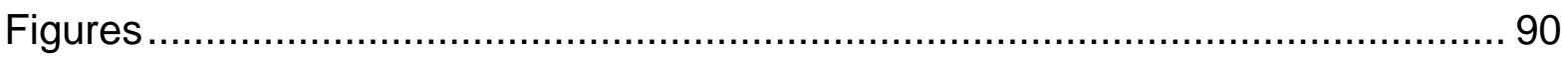

Evaluation of the Use of Methanogenesis to Covert Waste

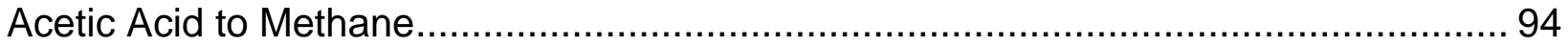

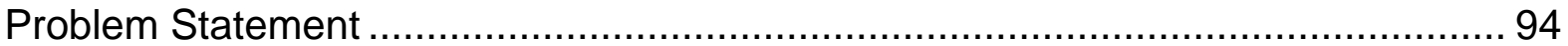

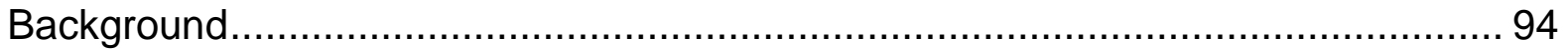

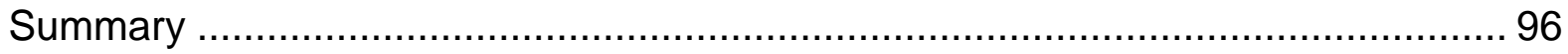

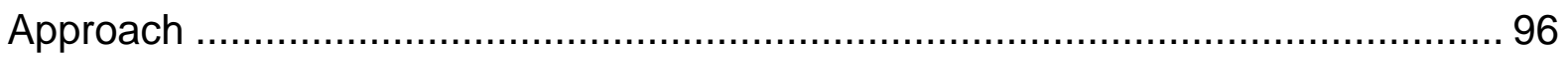

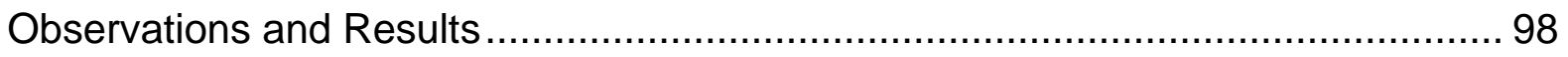

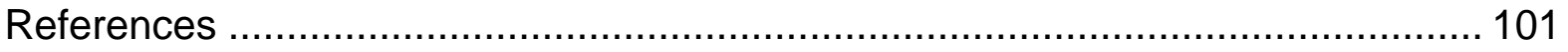

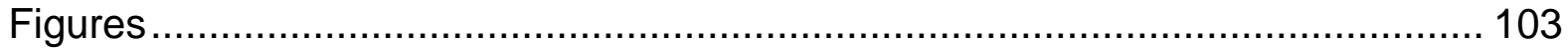

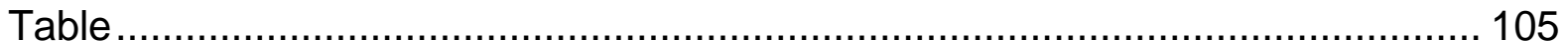

Methanogenesis at Low pH in Laboratory Scale Batch Fermentors.......................... 106

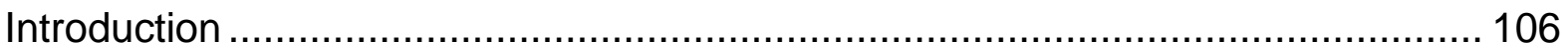

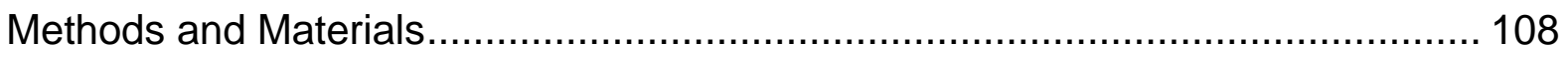

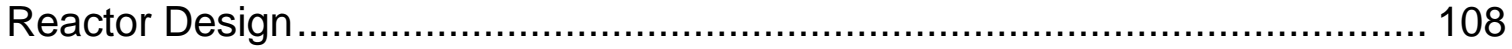

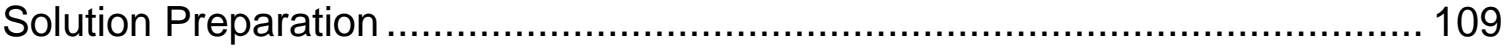

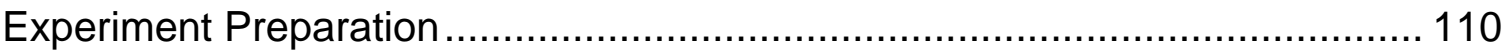

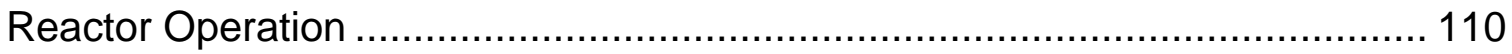

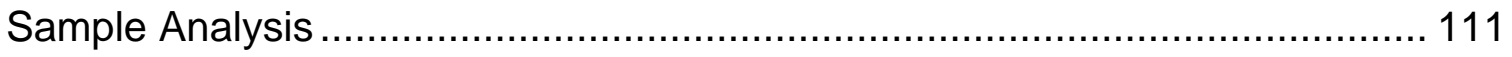

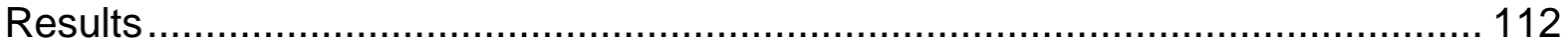

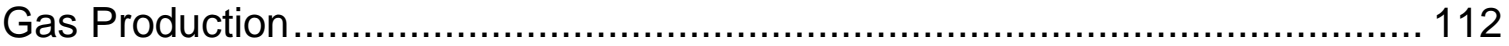

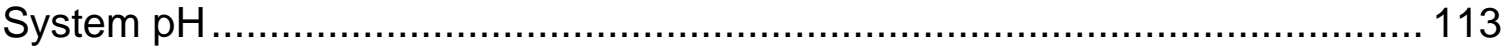

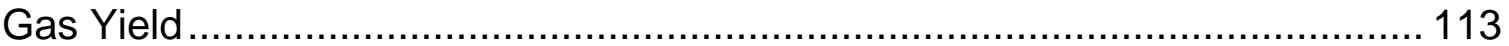

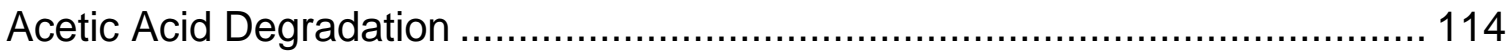

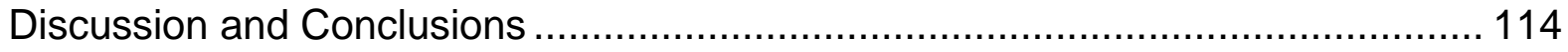

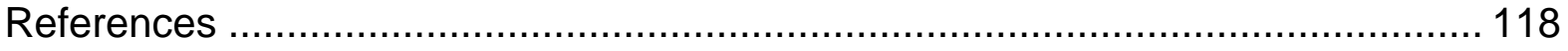

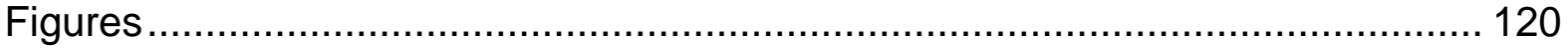

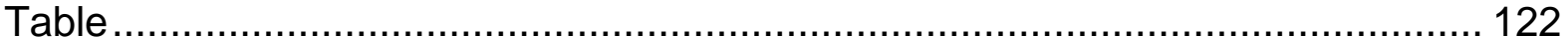


Effects of Selected by Products of an Acid Hydrolyzate on Cell Growth and Ethanol Fermentation by Saccharomyces Cerevisiae .............................................. 123

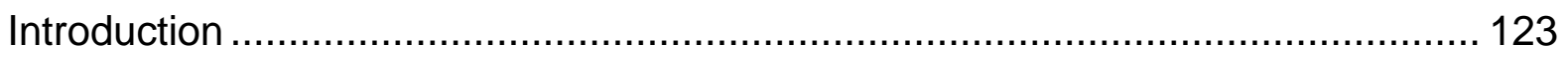

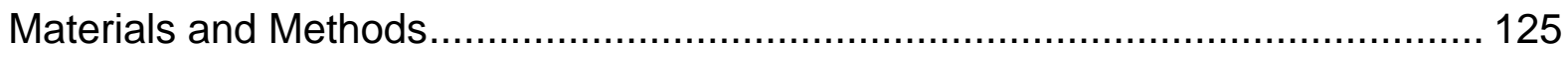

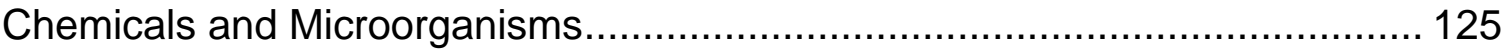

Effects of the By-products on Growth and Ethanol Production ....................... 125

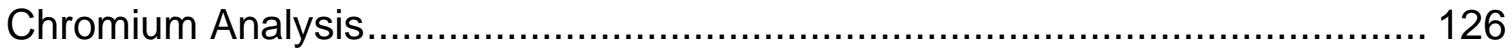

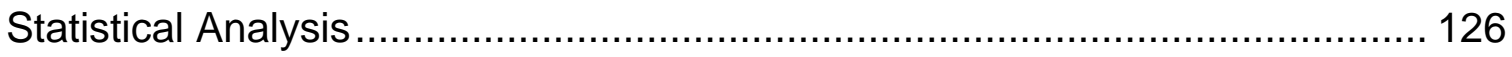

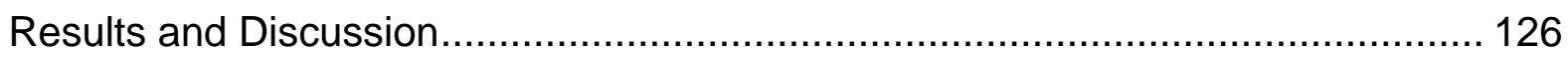

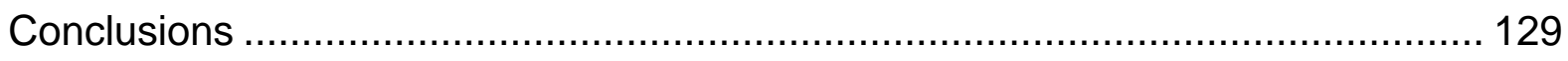

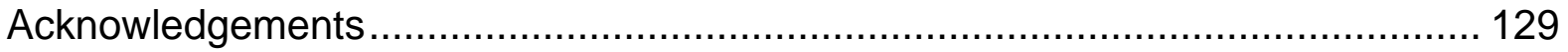

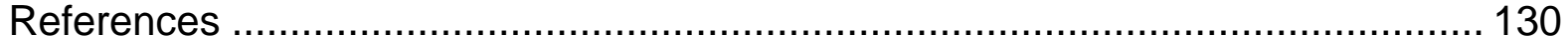

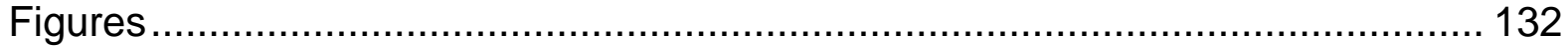

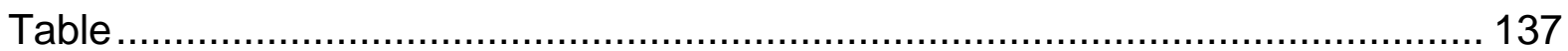

Enhanced Ethanol Production from the Fermentation of an

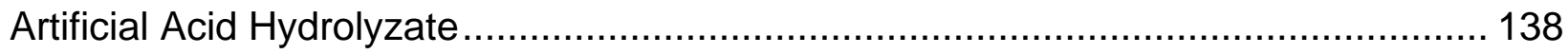

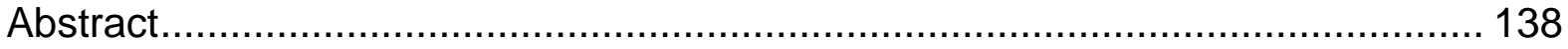

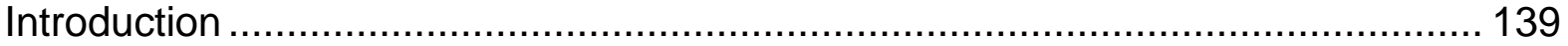

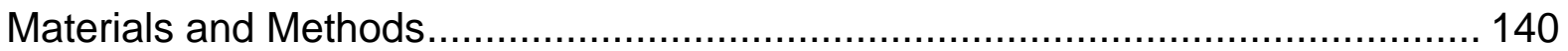

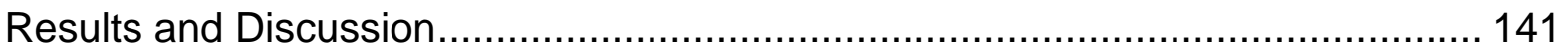

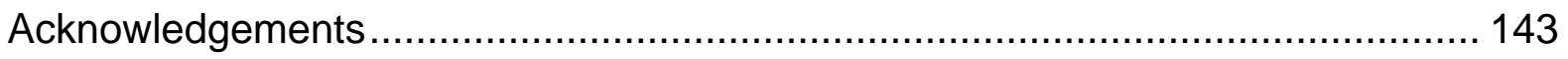

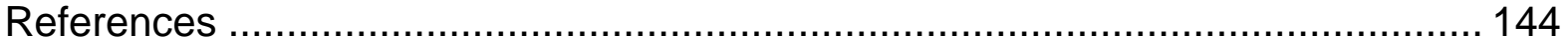

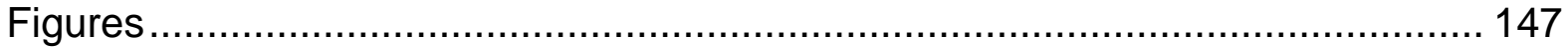

Enhancing Ethanol Fermentability of an Artificial Acid Hydrolyzate with Treatment of Anion Exchange Resins........................................................ 149

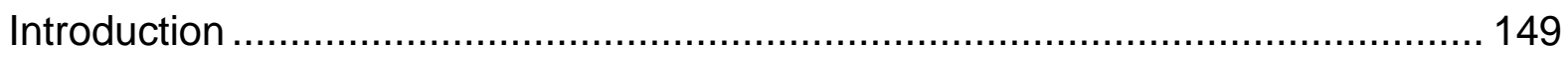

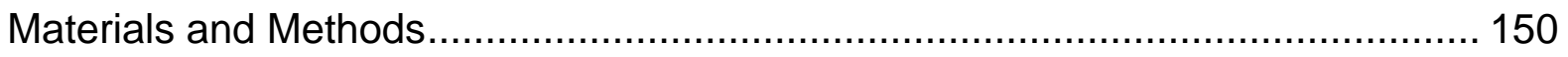

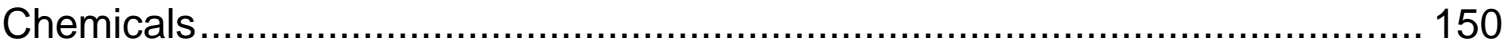

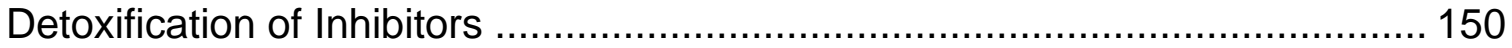

Effectiveness of Resins in Detoxification of the Inhibitor Cocktail................... 151

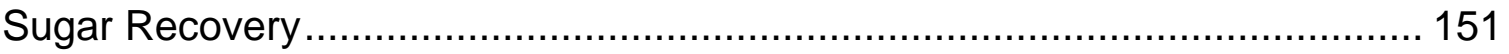




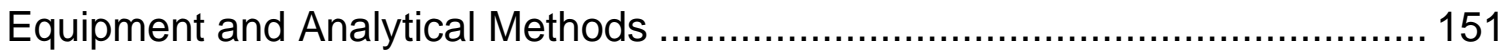

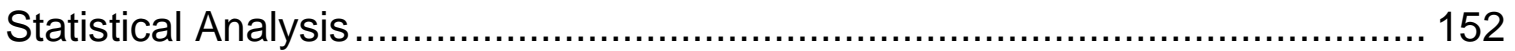

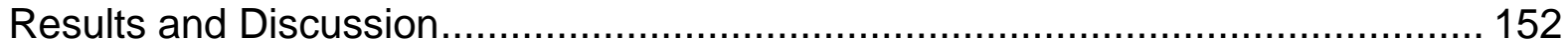

Detoxification of Inhibitors by Ion Exchange Resins ................................... 152

Effectiveness of Resins in Detoxification of the Inhibitor Cocktail................... 153

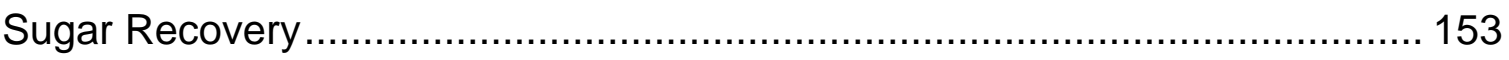

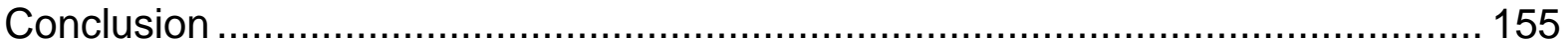

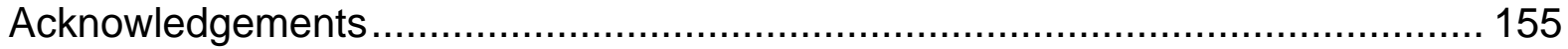

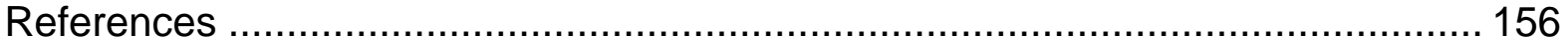

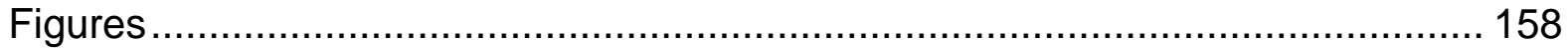

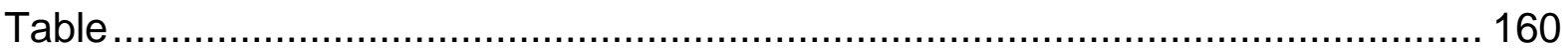

Membrane Separations Processes in the Conversion of Biomass to Fuels ................ 161

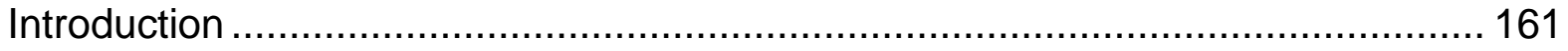

Membrane Processes for Acid-Sugar Separation of a Hydrolyzate Feed .............. 161

Membrane Processes for Ethanol Isolation and Dehydration ............................. 162

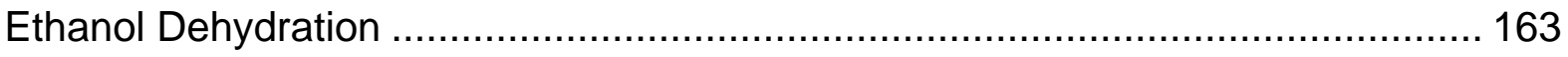

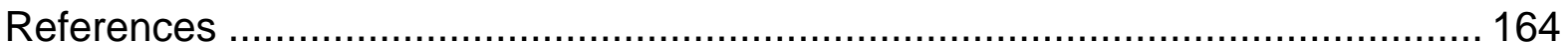

Development of Sulfonated Ethylene-Vinyl Alcohol Copolymers for

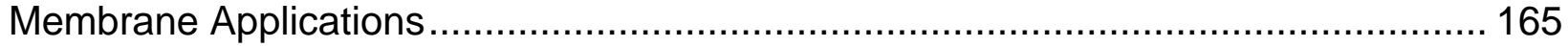

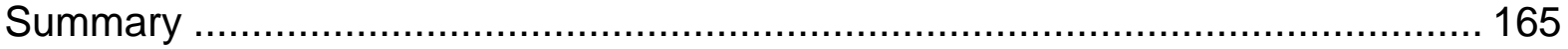

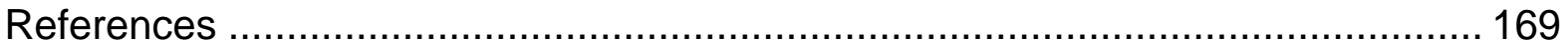

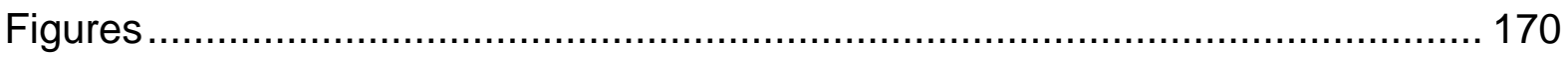

Acid-Sugar Separation Using Novel Membrane Systems ..................................... 175

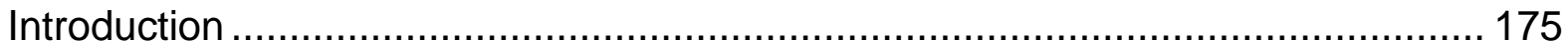

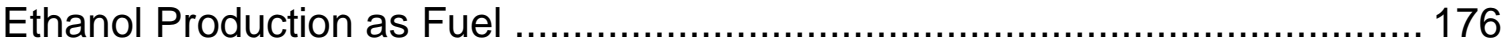

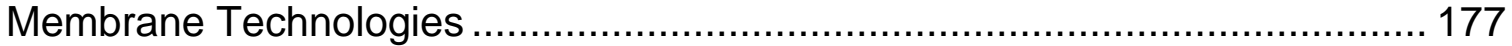

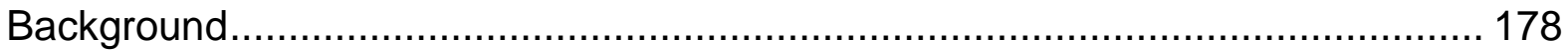

Ionomers as Membrane Materials ................................................................ 178

Preparation of Ion Exchange Membranes ……......................................... 180

Principles of Electrodialysis .................................................................... 183

Principles of Ion Exchange Membranes in Electrodialysis............................... 184

Ion Transport through Ion Exchange Membranes ....................................... 185

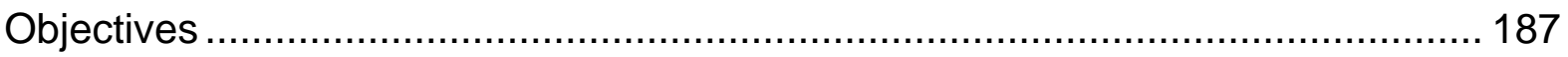




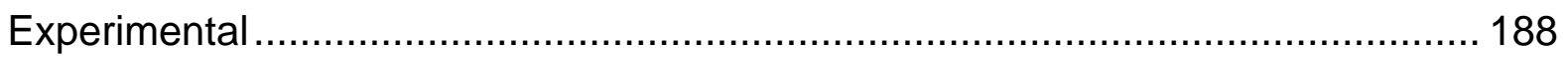

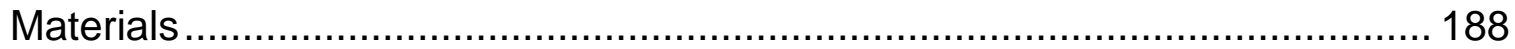

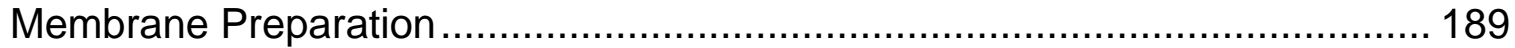

Synthesis of sulfonated ethylene vinyl alcohol ionomer ........................... 189

Crosslinking reaction of sulfonated ethylene vinyl alcohol ionomer............. 189

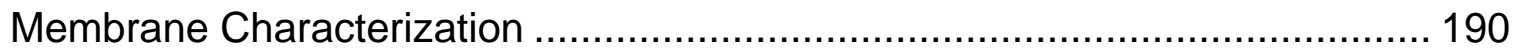

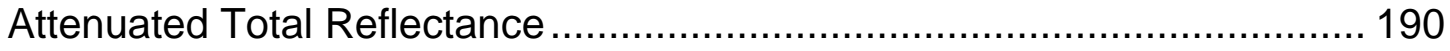

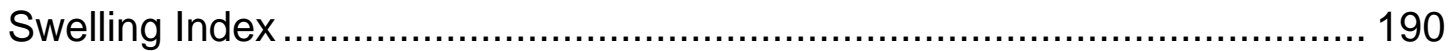

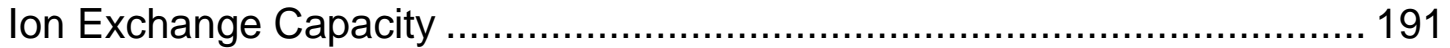

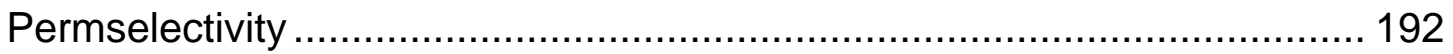

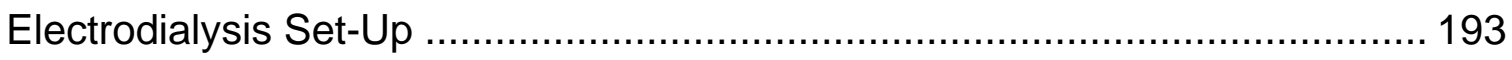

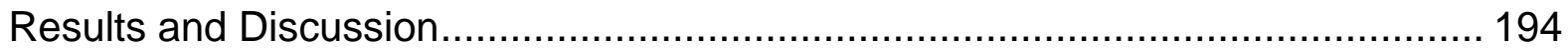

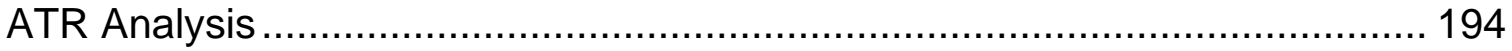

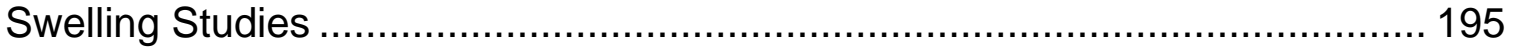

Ion Exchange Capacity and Degree of Sulfonation ..................................... 196

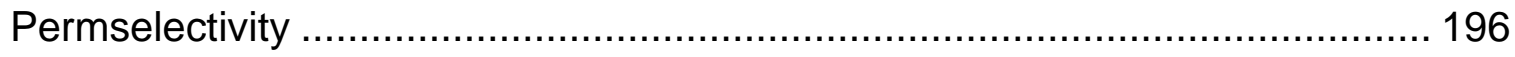

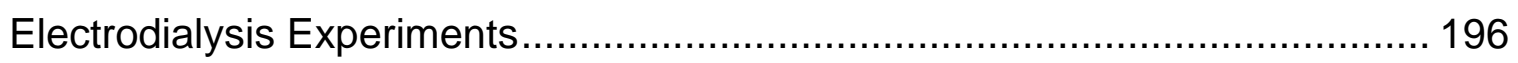

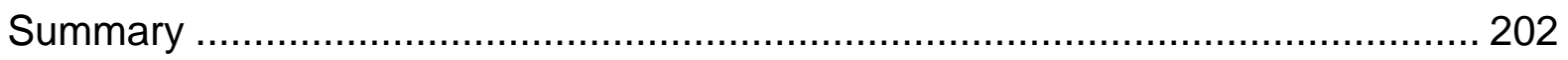

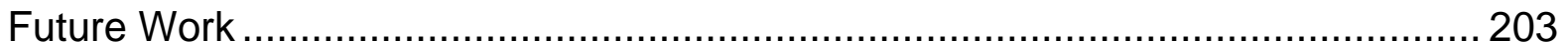

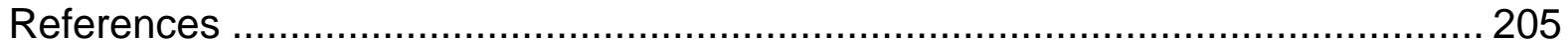

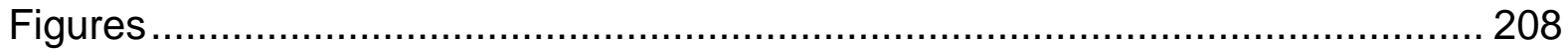

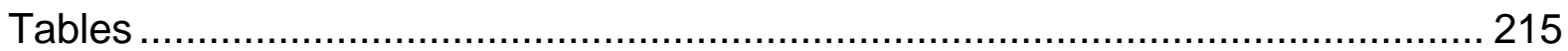

Development of a Concentrated Acid Hydrolysis Process to Continuously Convert Sawdust to Sugars that can be Fermented to Ethanol ............................................. 226

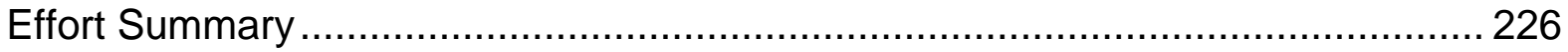

Cellulose Based Adsorbent Materials for the Dehydration of Ethanol Using

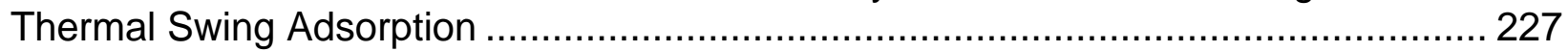

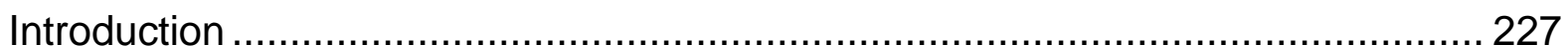

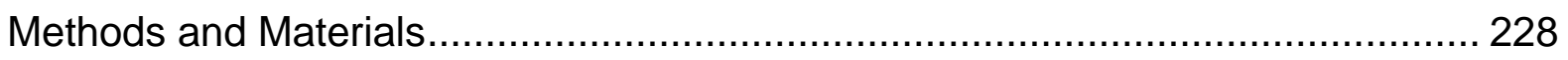

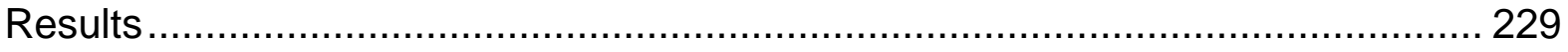

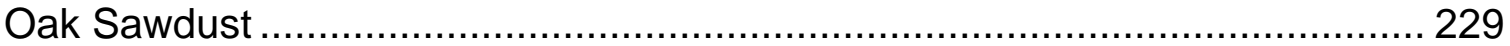

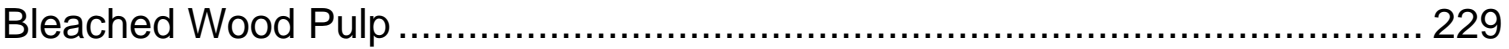

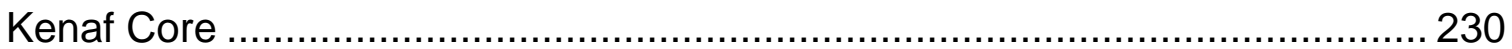




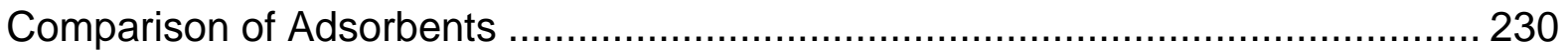

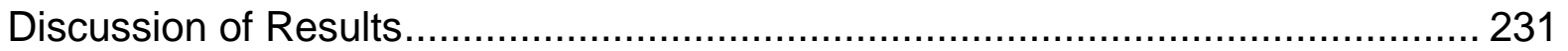

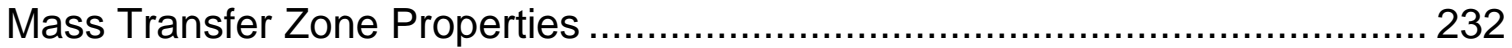

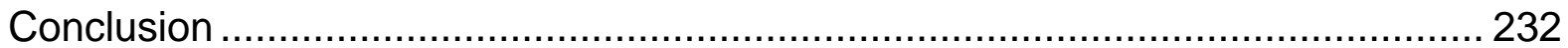

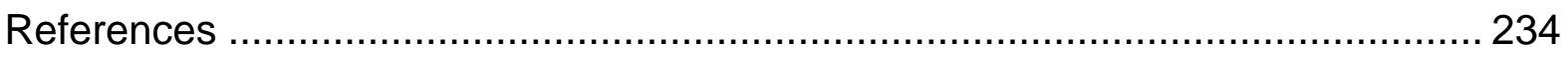

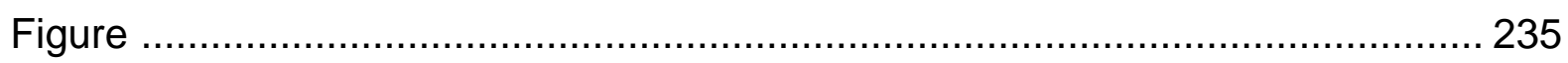

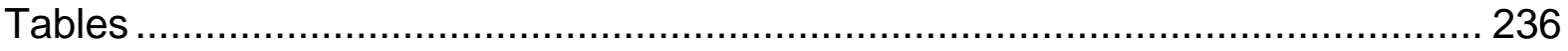

Development of a Perstraction Membrane Separation Method ................................ 238

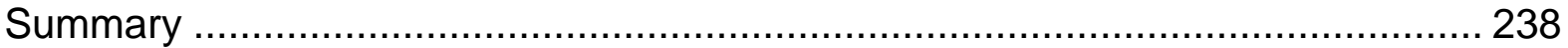

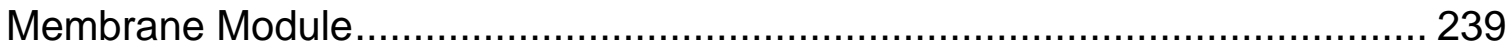

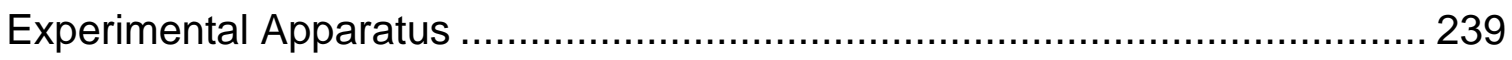

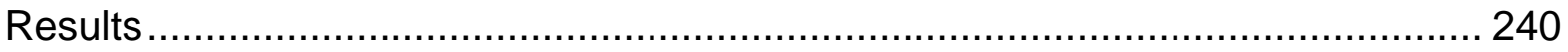

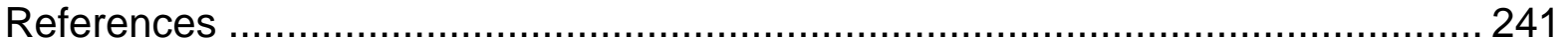

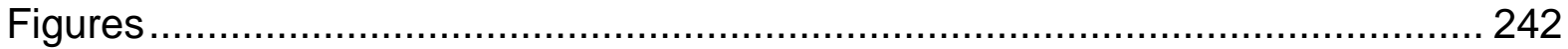

Production of Biodiesel from Wastewater Treatment Sludges …............................ 246

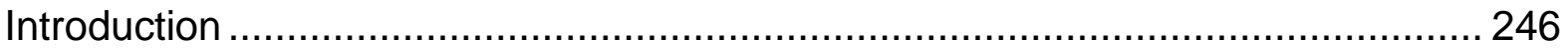

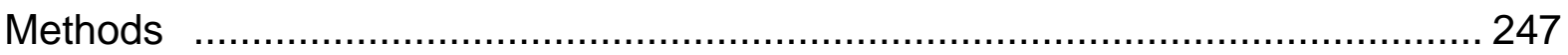

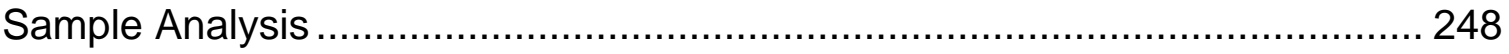

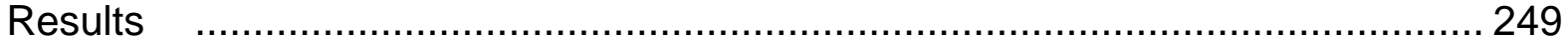

Efficacy of Different Solvents to Extract Oil from Sewage Sludge ................... 249

Effects of Sonication on Lipid Extraction ................................................ 250

Comparison of Solvents on the Types of Lipids Extracted ............................ 250

Comparison of Solvents on the Types of Fatty Acids Extracted ..................... 250

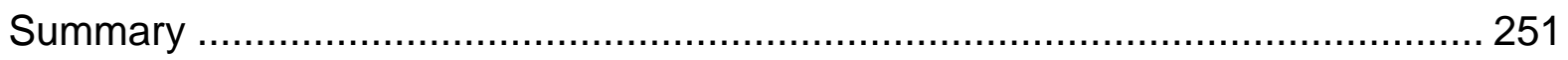

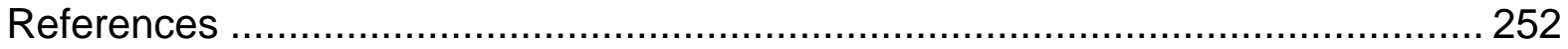

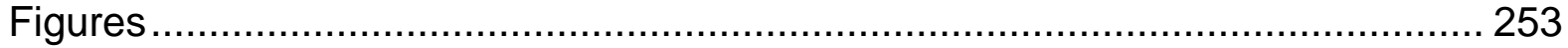

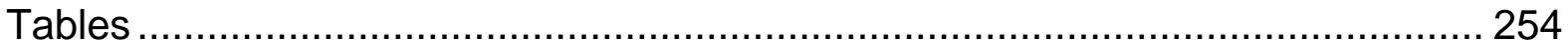

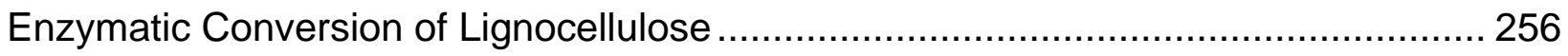

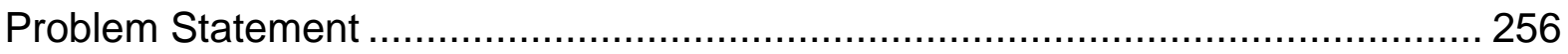

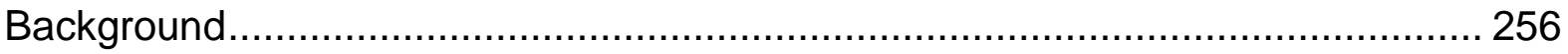

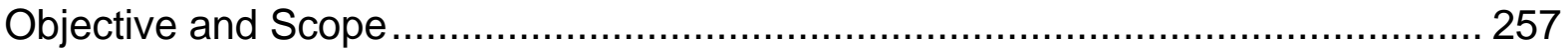

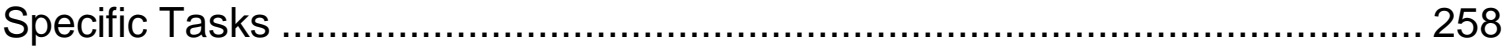

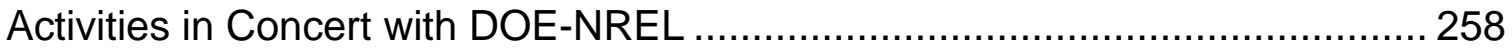




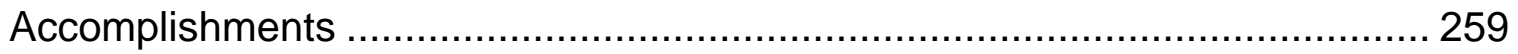

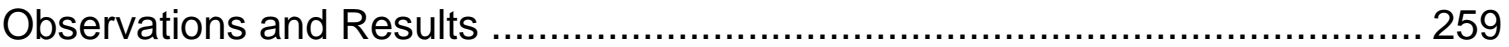

Analytical Characterization of Lignin Depolymerization by Fungal and

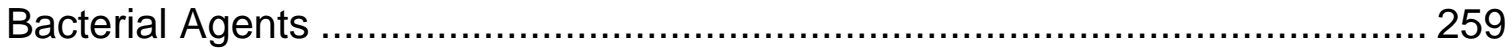

Molecular Analysis of Betsy Beetle Gut Flora ............................................ 261

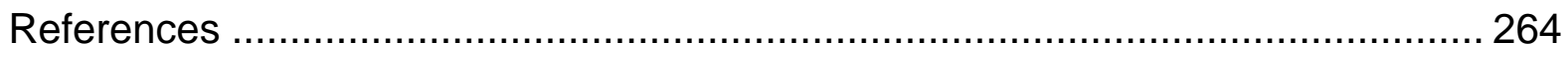

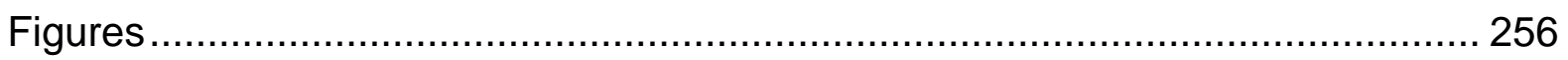




\section{SECTION I - INTRODUCTION}

Experts predict that mankind is quickly approaching an age where the production yield and overall inventory of fossil fuels, particularly petroleum and natural gas, have reached a point where they are no longer a viable energetic resource for societal needs. Hence, society must strive to develop an "energetic portfolio" of numerous contributing alternative energetic sources to compensate for eventual dwindling reserves of cheap petroleum. The three primary drivers for the need to find viable alternative energetic sources are home-front economics, national strategic independence, and simple resource availability. Clearly, the time is at hand when development of promising alternative energy sources must be accelerated and the most promising options fully commercialized.

The earth contains a wide variety of carbon reservoirs that can be harnessed to meet the societal power requirements in the form of gaseous, liquid, and solid fuels with liquid fuels being of most importance. The modern world has come to rely almost exclusively on fossil-based reserves, a non-renewable resource, for production of liquid fuels. However, the cost and politics of being totally dependent on these reserves is getting progressively more expensive from both a strategic and sociological standpoint. A renewable source of fuels is required for meeting the future energy needs of the United States and the world.

Unfortunately, politics and policy tend to be crisis management oriented resulting in a lag in the development of alternative fuels. The first real boost to the accelerated development of alternative bio-based fuels was President Clinton's Executive Order (released in August 12, 1999) that directed the Secretaries of Agriculture, Energy, and Treasury and the USEPA Administrator to initiate new research and stimulate industrial efforts on the development of alternative fuels. Since then, numerous federal and state initiatives have stimulated an unprecedented "boom" in both activity and funding for developing alternative energy sources. Of particular interest appear to be solar and biomass-based energy sources. Back in 1999, it was apparent that universities wanting to contribute to the developing national effort oriented toward producing liquid fuels from biomass, other than corn, needed to build capability in the form of both infrastructure and personnel. Hence, the project team proposed and received funding from the US 
Department of Energy (DOE)'s EPSCoR Program to form the "Mississippi Consortium for the Utilization of Biomass". This report summarized the activities and results generated over the funding cycle for the project which ran from Year 2000 through Year 2007.

\section{Biomass Derived Fuels and Chemical: The Vision}

The industrial revolution of the 1800's and 1900's was based on steel, fossil fuels, bricks, and mortar; materials which are now staples in America's industrial toolbox. However, most experts agree that the next industrial revolution, which is just beginning, will be based on biology-based materials or biomass. This revolution will usher in a totally new paradigm in terms of how society views both farm products and our organically rich wastes. It is envisioned that agriculture will diversify from producing only foodstocks to producing both crops for food and crops for chemical production. Chemical companies are investing tremendous monetary and intellectual capital in biotechnology that focuses on harnessing the vast chemical potential of plants. The result will surely be new relationships blossoming between the agricultural and chemicals production industries. Hence, the members of the Consortium teamed to form an expert base within Mississippi to assist the US in meeting its goal of energy independence using bio-based, renewable fuels while at the same time positioning these universities to be a collective resource for university-based $R \& D$ in the field.

Bioprocessing, the branch of biotechnology that uses biomass as industrial feedstocks, has evolved into one of the predominant positive new aspects of the alternative energy industry and arguably the overall chemical production industry. Whether it be ethanol, biodiesel, biogas, or hydrogen, biomass will very likely serve as the source of these valuable fuels.

The growth of the new field of bioprocessing, particularly with regard to the emergence of biofuels, mirrors the growth of the much more mature petroleum industry. In the mid-1800's, when petroleum was first discovered, the value of this strange black substance was not completely envisioned. By the turn of the century, a few chemical products were being produced from refineries. During the 1950's, refineries were producing twenty or so chemicals - primarily fuels. Today, the modern refinery produces well over thirty products with specialty chemicals bringing a large percentage 
of profits. In fact, literally tens of thousands of products used by society today come from petroleum. It took over 100 years to mature petroleum to the highly prized industrial feedstock it is today. It is interesting to see how petroleum slowly replaced coal as the energy source of the time. With regard to biomass, developmentally we are currently at the point in time that petroleum was at during the early 1900's. The term "biorefinery" has emerged more as a vision but some facilities are beginning to truly represent a facility best characterized by this term. Today's biorefinery typically produces fewer than five chemicals - mostly bulk chemicals and few specialty chemicals. It is believed that tomorrow's biorefinery will produce a significantly increasing number of products ranging from bulk fuels to valuable specialty chemicals to pharmaceuticals. Efforts such as the R\&D activities performed by the Consortium will play a long-term part of the rapidly developing area of Biorefineries. Without the benefit of the EPCoR program funding, it is doubtful that the team members would have evolved to the level of expertise and resulting activity that they enjoy today.

\section{Potential for Mississippi to Become a Leader in Biofuels Production: A Perspective}

Mississippi is a leader in agricultural and forestry activity (gauged in terms of production volume, percent land utilization, and per capita involvement). Many of these activities produce vast quantities of biomass that are simply managed as a waste disposal problem and not viewed as an energy resource. The wood products industry in Mississippi is the largest manufacturing sector in the state, employing over 65,000 people, with a raw materials stock estimated by Mississippi State University in 1999 to be over 3.1 billion dollars. One in every four jobs in Mississippi is forest product related. These activities include both raw lumber and wood product manufacturing (Mississippi is the second largest furniture producing state). Over 4 million tons of wood product waste annually are not utilized for any beneficial product development and thus require costly disposal. Mississippi is also a one of the nation's top producers for poultry; as such, it produces a tremendous amount of biomass-based litter. Statistics indicate that much of Mississippi potentially valuable biomass resources are being landfilled or opendumped into uncontrolled waste pits and open areas. In essence, Mississippi is wasting valuable fuel feedstocks by paying to dispose of them or contaminating the environment 
with these high biochemical oxygen demand (BOD) laden wastes. This predicament is not unique to Mississippi or the United States. The wasting of biomass is a world-wide energy loss and environmental disposal problem.

For all of the agricultural activity in Mississippi, there are still numerous acres of agricultural land in Mississippi not being used for crop raising because they are of minimal agricultural quality. Recent unpublished work by the Mississippi Agricultural and Forestry Experiment Station (MAFES) indicates that these lands are well suited for supporting the growth of high density alternative crops such as switch grass and other grass varieties. Their results indicate that Mississippi could be a leader in biomass growth for ethanol production due to its climate and transportation system. This is good news for entities within Mississippi interested in producing ethanol since corn is not the most optimal crop for Mississippi's climate.

It is envisioned that centralized ethanol production facilities can be located throughout biomass production areas in the Southeastern US which will convert the disposal problem detailed above into a utilizable and profitable fuel source. A developing fuel production industry based on cultured biomass feedstocks, such as grasses, using the proposed technologies will also offer new uses for the medium to low quality agricultural acreage which cannot be used very successfully for the cultivation of challenging to grow crops, such as corn. Since many of these areas are among the poorest regions of the United States, a much needed economic shot-in-the-arm would be delivered to these economically impoverished areas with the realization of this vision. One final note is that a significant portion of the industrial base present in Mississippi and Louisiana is heavily oriented toward petroleum refining, allowing for easy implementation of ethanol-gasoline blending as policies and incentives change.

\section{Overall Project Goals and Objectives}

The production of biofuels has matured from a niche market into a true industry within the US. The biofuels industry has emerged from one producing only a few million gallons per year to an estimated yield in 2007 in excess of 5 billion gallons. This growth cannot be sustained unless the chief production feedstocks are shifted from food-stocks grown on high quality farmland to chemical crops grown on marginal land. To date, biofuels-oriented R\&D has been almost exclusively oriented toward crops from the Mid- 
western US. For the US to develop a highly productive portfolio of biofuels production to truly displace petroleum-based fuels significant R\&D efforts must be directed toward the other regions of the US which are different from an agricultural perspective, yet as capable, or in the case of the Deep South even more capable, of producing more tonnage per year of biomass than the Midwest. The Present and both major political parties continually point to biofuels and related technologies as one of the key industrial developmental challenges facing the US. The industry has evolved from one where selling the product was the primary challenge to one where finding technically and economically viable feedstocks is a key concern obstructing the real potential of these technologies to solve ever-growing global energy concerns.

The over-arching goal of this project was to develop a nationally competitive, multi-university research entity within Mississippi capable of positioning the state as a leader in the biofuels and biochemicals arena. Of particular interest was the development of a technological foundation would position Mississippi as a leader within the Southeastern US in biofuels production. Hence, a multi-faceted approach was carefully designed to meet this overall goal. The following secondary goals to support this vision were developed:

a) Explore chemical processing techniques that well-matched existing Mississippi biomass feedstocks for production of ethanol from lignocellulosic sources.

b) Develop processing techniques used in producing biomass-derived fuels that are optimized to improve both technical and economic performances.

c) Assess process residual and associated manufacturing techniques that may be used to produce secondary value-added products as a means of increasing profitability

d) Utilize the above goals to coalesce a team of accomplished researchers from the four Research Universities within Mississippi who have considerable accomplishments as small independent groups into a united research team capable of competing for large federal grants, foster industrial collaborations to help site the blossoming number of bioproduction facilities going up in the US within Mississippi, and to potentially spin-off new start-up companies for commercialization of university-generated IP.

e) The formation of a "pipeline" of technologists for supporting the biofuels and bioproducts industry within the state and the US through the graduation of scientists and engineers skilled in the area of bioprocessing. 
f) The infusion of significant industrial interaction with the university research team to increase the potential for producing technologies that are easily transitioned into commercial realities and to provide a developmental foundation to these companies are they attempt to further mature the bioprocessing industry; particularly, with regard to issues related to the Southeastern US.

\section{Overview of Research and Development Efforts: The Experimental Design}

This section summarizes the R\&D activities undertaken by the project team over the project life. Note that these topics were selected by the Consortium members based on many factors, including potential to enhance economic development within Mississippi, R\&D areas that the Consortium members felt would continue to be of national interest (hence, have long term funding potential), institutional resources and capabilities already in-place, input from program reviewers provided over the contract life by the US DOE, and the potential to expand faculty participation within each university. With that said, due to the efforts initiated as part of the DOE EPSCoR support, numerous other R\&D projects were initiated using the Consortium's support as the foundation for these new projects - many of which were funded via other funding mechanisms (federal, state, institutional, or private).

\section{Key Research Topics Evaluated During the Project}

The following sections highlight the various process development efforts undertaken by the project team as a means of converting the candidate processes from conceptual designs with dubious economic potential to economically viable processes which can be utilized within rural areas that are currently producing high volumes of waste and/or cultured biomass. These rural areas in Mississippi can be transformed from areas undergoing public debate over waste disposal issues to economic development zones producing biofuels and bioproducts as industrial products. The following topical areas of research were the focal point of the Consortium's activity:

- Fermentation of Syngas into Ethanol

- Production of Ethanol via Acid Hydrolysis

- Production of Novel Polymers for Potential Use in Bioproduct

- PurificationProduction of Biogas from Wastewaters

- Production of Chemical Building Block Chemicals from Lignin

- Production of Biodiesel from Waste Solids 
A summary description of each research focal point researched by the Consortium is briefly discussed below:

\section{Fermentation of Synthesis Gas}

DOE funded significant pioneering research during the late-1900's which focused on the utilization of an autotrophic fermentation process for the conversion of waste gases from refinery operations into usable products, such as ethanol and acetic acid/acetate. The sources of these gases included the following refining unit operations: cat crackers, cokers, and catalyst regeneration units. Additionally, the fermentation of synthesis gas has been evaluated. Synthesis gas (or syngas) is a common gas product produced from reformed natural gas (done catalytically) or from coal gasification. Syngas has been used to produce secondary fuel products and primary alcohols. Most of the primary alcohol work has been focused on the production of methanol using heterogeneous catalysts including ruthenium, moly-sulfide, and nickel based units. The composition of these gases is generally hydrogen $(\sim 50 \%)$, carbon monoxide ( $25 \%)$, and carbon dioxide ( $25 \%)$.

The microorganisms that were isolated from the past DOE ethanol from syngas efforts were found within the waste pits of poultry raising operations by researchers from the University of Arkansas working under a DOE grant. It was reported that further culturing and acclimation resulted in the development of advanced strains that were derived from the wild strain. The mechanism used by all of these organisms for the production of ethanol is:

$$
\begin{aligned}
& 6 \mathrm{CO}+3 \mathrm{H}_{2} \mathrm{O} \quad \mathrm{B}>\mathrm{CH}_{3} \mathrm{CH}_{2} \mathrm{OH}+4 \mathrm{CO}_{2} \\
& 6 \mathrm{H}_{2}+2 \mathrm{CO}_{2} \quad \mathrm{~B}>\mathrm{CH}_{3} \mathrm{CH}_{2} \mathrm{OH}+3 \mathrm{H}_{2} \mathrm{O}
\end{aligned}
$$

The above reaction scheme is reported to yield approximately $25 \mathrm{~g} / \mathrm{l}$ of ethanol within the aqueous effluent exiting the fermenter which can be recovered as anhydrous ethanol using distillation and adsorption. It has also been reported that the carbon monoxide reaction is much more productive in terms of ethanol production. This mechanism is also more stable from a cell growth standpoint which allows for increased biocatalyst production, hence the higher yields of ethanol.

Studies indicate that under fermentation conditions favoring ethanol production, acetic acid is also produced. Approximately $5 \mathrm{~g} / \mathrm{l}$ of acetic acid is formed following the 
mechanism detailed below where approximately $25 \mathrm{~g} / \mathrm{l}$ of ethanol is being produced within the same fermenter (5:1 product yield):

$$
\begin{aligned}
& 4 \mathrm{CO}+2 \mathrm{H}_{2} \mathrm{O} \quad \mathrm{B}>\mathrm{CH}_{3} \mathrm{COOH}+2 \mathrm{CO}_{2} \\
& 2 \mathrm{H}_{2}+4 \mathrm{CO}_{2} \quad \mathrm{~B}>\mathrm{CH}_{3} \mathrm{COOH}+2 \mathrm{H}_{2} \mathrm{O}
\end{aligned}
$$

Currently, the acetic acid formed within the fermentation step ends up as the major component of the aqueous stream produced from the ethanol separation step (as a distillation bottoms). This stream is often considered a wastewater to be treated within a treatment plant prior to discharge. Alternative uses of the acetate within the fermentation process, such as recirculation, was evaluated by others with little potential noted because of alcohol production inhibition within the fermenter. It is interesting to point out that slight modification of the fermentation process does result in a much higher yield of acetic acid with little ethanol being formed (this mechanism is likely to be of interest to federal agencies such as USDA rather than to DOE).

Hence, from this scientific knowledge base, the efforts within this effort were almost exclusively oriented toward novel fermenting organism discovery and determination of novel reactor conditions and design to support the performing organisms.

\section{Ethanol Production Via the Fermentation of Acid Hydrolyzate}

Acid hydrolysis of waste biomass containing appreciable percentages of cellulose and hemi-cellulose has been under development for several years. Conversion that essentially can be described as two step process is well known: conversion of cellulosic materials to sugars followed by fermentation of the sugars to ethanol. As is the case with the fermentation of syngas, acid hydrolysis is much more forgiving in terms of biomass feed composition than cellulase-based techniques because of the aggressiveness of the acid toward hydrolysis as opposed to problems with enzyme specificity. Problems do persist with costly high acid loss and heterogeneous introduction of the acid into the physical matrix of the waste biomass.

From the early years, the Tennessee Valley Authority (TVA) biomass development program renewed research previously carried out at the USDA Peoria Laboratory on converting agricultural waste into useful products by using an acid 
hydrolysis extraction/conversion process. TVA, with assistance from Mississippi State University, built a 4-ton-per-day concentrated acid hydrolysis experimental facility at Muscle Shoals, Alabama in 1985. In this concentrated acid process, cellulose and hemi-cellulose from hardwoods were reduced by sulfuric acid to xylose and glucose sugars. Unused acid was removed from the sugars by lime precipitation. The sugars were then fermented to ethanol. Process concepts were demonstrated and high hemicellulose and cellulose conversion efficiencies were achieved. However, process economics were poor as a result of two major problems: (1) the acid was continuously consumed, elevating operating costs, and (2) equipment costs were too high for a favorable investment return. Between 1993 and 1998, The University of Southern Mississippi and TVA researchers continued to improve the concentrated acid process. A twin screw extruder reactor was utilized for low temperature continuous impregnation of hardwood sawdust with sulfuric acid. The shearing action of the extruder's corotating screws increased acid exposure to the biomass structure by continually removing acid treated biomass surface, thereby exposing new surfaces to the acid. A tube reactor was used to convert the hydrolyzate stream from the twin screw extruder into glucose and xylose sugar. Continuous ion exclusion techniques were perfected so that sulfuric acid could be separated from the sugars formed in the tube reactor. The acid stream from the separation step was concentrated and thereafter reused. The sugar stream from the separation step is then fermented into ethanol. Ethanol can be produced by microbial fermentation of the sugars derived from acid hydrolysis of lignocelluloses. However, a key factor in the conversion of sugars to ethanol is finding suitable microorganisms. There are several desirable characteristics for selecting the microorganisms to achieve this mission. The microorganisms should produce high ethanol yields, have high ethanol tolerance levels, have resistance to acidity and toxicity of hydrolyzates, have no or low oxygen requirement, and a broad substrate utilization capability.

Based on this state of the science, the Consortium members decided to direct research toward both the reactor and the study of the performing microorganisms within the hydrolyate fermenters. The reactor work involved modeling governing reactions and using this new level of knowledge to improve hydrolysis effectiveness within improved 
reactor designs/operations. Numerous microorganisms were evaluated and the relative impact of hydrolysate conditions studied to evaluate the sensitivity of the organisms toward the impact of hydrolysate constituents on key biochemical reactions.

\section{Biogas Production from Wastewaters}

Another intriguing research issue that addresses a very practical problem facing potential commercial development of syngas fermentation is the management of the acetic acid formed as a secondary fermentation by-product (see above discussions). It is believed that treating an acetic acid laden wastewater (estimated to be approximately $5,000 \mathrm{mg} / \mathrm{l}$ ) containing this high BOD is an expensive option and waste of a valuable carbon source which may be used for secondary production of a potentially beneficial product. Various extraction/recovery techniques are believed to be technically and, possibly, economically feasible for recovering the acetic acid as a secondary product. Examples of potential processes to be evaluated include advanced distillation, liquidliquid extraction, membranes, or a combination of these.

A management option for the acetic acid in the waste stream from ethanol separation researched by Consortium members was the anaerobic digestion of the process wastewater to produce methane (via decarboxylation) which can be used as an energy source for firing the distillation secondary burner or used as a fuel within the syngas facility. Past research had indicated the various digester systems were capable of producing methane from "elevated" levels of acetic acid (albeit, much lower than the stream expected from syngas fermentation). These results were generally obtained from traditional systems with little attention paid to the overall process rigor of the system.

Germany has implemented a massive biogas production effort (greater than 400 plants) from agricultural manure treating facilities which is reportedly successful in terms of methane recovery and utilization (Business newsletter 1998). Anheuser-Bush is currently feeding brewery wastewaters into an anaerobic digestor that is producing methane from an estimated 90,000 pounds of BOD yielding a reported fuel cost savings of $\$ 485,000$.

Aerobic biotreatment is the most popular waste treatment technique for industrial wastewaters. However, unpublished studies at MSU indicate a high BOD mass 
conversion rate from sodium acetate which represents a relatively high organic loading that will make aerobic biotreatment too costly for the management of the wastewaters produced by the current designs for ethanol-from-syngas facilities. Therefore, it likely some form of anaerobic treatment must be implemented; however, from an economic standpoint, this approach is best when coupled with methane recovery. Therefore, a high potential does exist for taking the process acetate waste stream and converting it into a source of additional energy in the form of biogas. Utilizing this acetic acid will greatly reduce the organic loading of down-stream wastewater treatment processes. At worst, if sufficient methane cannot be produced, this effort will provide an evaluation of the potential for using an anaerobic bioreactor system for treatment of the acetate laden stream, which is considered an economically more attractive waste treatment option than aerobic biotreatment for this type of wastewater.

The primary research attention of the Consortium projects in this area was directed at stressing traditional sources of active digestion organisms in terms of high levels of acetic acid feeds, reactor operational upsets, and methods to initiate or reestablish stable operations using chemical amendments. Additionally, a series of novel laboratory screening methods were developed by MSU and Ole Miss to facilitate rapid screening of anaerobic digester operations.

\section{Development of Novel Polymers for Use in Production Separation}

The benefits of acid recovery and reuse in the conversion process are both economic and environmental. The present technique for acid/sugar separation uses a continuous ion exclusion chromatography system which provides effective recovery of the acid and sugars. However, the ion exclusion separation process, while effective, requires complex equipment and control systems to achieve high efficiency. A review of available literature indicated the overall feasibility of using a less expensive membrane based separation system. Thus, it was viewed that research was needed to investigate the membrane micro-architecture required to separate hydrolyzate into its components, acid and sugar.

The chromatography system used successfully in past research to separate hydrolyzate components made use of sulfonated polystyrene materials. The membranes proposed to be developed in this study for extracting sugars from the acid 
hydrolyzate will be fabricated from the same type polymeric materials. However, because it was not possible to predict the necessary degree of sulfonation for successful sugar separation, a range of degrees of sulfonation in the membrane was explored. This involved using two membrane formation approaches: post-polymerization sulfonation and co-polymerization. Post-polymerization techniques could be applied to solvated polymers or pre-fabricated porous membranes. This allows the study of the differences in membrane formation as a function of ionic group content and the effect of the sulfonation reaction on the membrane. Secondly, it is possible to create copolymers from styrene and styrene sulfonate. These copolymers allow a much larger variation in sulfonate group content in the polymer than is obtainable via post-polymerization reactions. The use of a more conventional polysulfone matrix as an alternative membrane material will be evaluated. These materials may be sulfonated using procedures similar to that used in the preparation of sulfonated polystyrene. Manufacturing processes can be customized to produce asymmetric membranes with a "swiss cheese" or fingered morphology. Asymmetric fingered membranes can be visualized by imagining a porous material with conical pores. The excluded material is larger than the narrow inlet channel opening and is therefore prevented from entering the membrane. However, once the permeate passes through the narrow inlet opening, flow is unrestricted by the ever widening channel passage. This feature of an asymmetric membrane eliminates the problem of blocked pores inherent to symmetric membranes. Due to the number of experimental variations, experimental and predictive models already developed by USM were employed to narrow the range of possible membrane structures, including porosity of the membrane, degree of sulfonation, and pre- versus post-polymerization sulfonation. The use of statistical experimental design techniques allowed a large number of membranes to be evaluated and direct the research to a micro-architecture that gives optimum acid separation.

At the initiation of this project, the most important polymeric membranes for separation processes were those made by a phase inversion technique. The phase inversion technique is based upon the addition of a non-solvent to a thin layer of solvated polymer and can result in the formation of microporous membranes with variable morphologies. The mechanism of formation is a complicated combination of 
mass transport and thermodynamic phase separation phenomena. The non-solvent induced phase inversion (NIPI) can be accomplished in a variety of ways that are detailed within research databases.

Another process deemed of interest for membrane fabrication was thermally induced phase separation (TIPS). In this process, both metastable and unstable phase regions are first identified. Of the three possible morphologies that can be developed, those derived from the unstable regions are of the most interest. Phase separation through an unstable region can result in membranes that have both uniform interconnected pores and good mechanical strength.

Phase change separation processes were also evaluated because of the importance of developing an energy efficient acid concentration process for minimizing operational cost. Sulfuric acid concentration by multi-effect evaporation were investigated. Multi-effect evaporation is a process whereby the dilute acid solution from acid recovery can be concentrated in several stages or effects with the vapor generated in each effect being used as a heat source in each succeeding effect. This process allows heat efficiencies of five or six times greater than single stage evaporation. This method of concentration by multi-effect evaporation is used extensively in the desalinization of sea water and in paper mill black liquor recovery processes. However, because of corrosion difficulties, its application to sulfuric acid concentration presented a unique set of challenges.

Adequate removal of acid from the sugar feed stream to fermentation is critical to the survival of the fermenting organisms. However as acid levels are reduced in the sugar stream output, all separation processes become less economical. Thus, the development of acid tolerant sugar fermenting organisms would reduce the demands on any acid/sugar separation process and provide increased robustness to any variability occurring in the acid-sugar separation system.

Much of the developmental work initiated by the Consortium members (primarily, USM) is detailed above. In essence, the key goal was to reduce the cost of ethanol production via improved separation; however, a side benefit proposed was the expansion of the state of the art within the field of membrane separations. 


\section{Enzymatic Conversion of Lignin}

Extensive research has focused on fungal degradation of wood components via enyzmes, such as cellulases and ligninases. Much of the work by done others thus far has focused on feedstock pretreatment, reduction of enzyme costs, and the environmental uses of ligninases for treatment of complex organic substrates. Currently, DOE has an extensive research program underway to field a commercially viable cellulase-based process. The most often cited overall concept is the conversion of hemi-cellulose into cellulose and/or sugars via a dilute acid hydrolysis. Cellulose fractions are converted by cellulases into sugar. The converted sugars are then fed into a fairly traditional fermentation step for conversion of sugar into ethanol. Currently, the lignin is not utilized for production of value-added chemicals. However, it still represents a significant portion of the total mass of the feedstock carbon. Therefore, using lignin as a convertible feedstock would further improve the economic outlook of both acid hydrolysis and enzyme conversion.

During degradation of wood, fungi utilize enzymes to attack both lignin and cellulose. Of the two enzymes, lignin has been the most under-studied from a potential chemical production standpoint. Cellulases are already used in industry; however, the cost of the enzyme hinders its use for ethanol production. Discussions with scientists from NREL have indicated numerous potential functions that lignin may serve for producing chemicals of commercial value. NREL, along with the University of Minnesota, is currently supporting research on lignin depolymerization by a strain of the fungi, $T$. cingulata. Results have indicated depolymerization. However, further work is underway to confirm this and to generate enough of the enzymatic protein for genetic analysis. Sophisticated separations technologies involving size exclusion chromatography and light scattering photometry are being used to assay products.

Fungi require high oxygen concentrations and use ligninases (peroxidases) that generate high valence state manganese ions as the nonspecific agent for disrupting the lignin polymer. Little work has been done on other than fungal sources for production of key fungal-based enzymes such as ligninase from gut ecology. A need has been identified for research to investigate alternate agents (e.g. bacterial) capable of bond 
scission, with particular focus needing to be placed on determining the relationship of lignin degradation and redox conditions for these agents.

Where lignocellulosic materials are utilized in nature for carbon and energy, more than one type of microbe are engaged in their degradation. A group of microbes, often of very different physiological and phylogenetic types is referred to as a consortium. The rate or the fate of the degradation without the activity of the consortium would be limited or absent. Many insects have specialized in the degradation of lignocellulosic materials. Most of this research has focused on the wood-eating termites. Various beetles, cockroaches and the larvae of many other insects have also specialized in utilizing these materials as food. Within the past few years, it was learned that a substantial part of the termite gut is aerobic or microaerophilic. This explains the possibility of the contribution of oxygenases in the degradation of the lignin matrix. A portion of the gut is anaerobic as well - so the gut could be considered a gradient oxidation-reduction potential environment. We furthermore understand that the gut is dominated by bacteria and protozoa (often with bacterial symbionts). The presence of fungi is greatly reduced. Several organisms have been isolated that have the potential to catabolize key functional groups in the dismutation of the lignin superstructure, although no one bacterium has been shown to degrade lignin. A sharing of catabolic potential is much more energetically beneficial and contributes to process stability. It is probable that aspects of both the aerobic and the anaerobic carbon cycle are critical to termite survival.

Lignin is a complex biopolymer. Lignin conversion involves complex reactions of bond scission and functional group alteration. There is a research need to assess and quantify what conversion means; to determine what conversion products are; and to better understand different functions being carried out by enzymes-both specific and general (non-selective bond scission). There is a need to characterize lignin conversion $B$ in terms of quantifying lignin depolymerization, disengagement of lignin from cellulose, and ultimate conversion of cellulose to ethanol. Regarding engineering issues, earlier research supports the wet storage and shipment of agricultural biomass. In seeking organisms and exploring conditions, one should keep this storage scenario in mind. It offers advantages for enzymatic conversion over long periods (months) of time. 
It also presents challenges for use of organisms requiring highly aerobic conditions $B$, an issue our planned research addresses.

The efforts in this area were done in direct collaboration with NREL. Much of the work was directed toward functioning bacteria discovery and the evaluation of methods to utilize the observed novel degradation capacities to potentially produce chemicals of industrial interest.

\section{Production of Biodiesel From a Novel Feedstock}

Biodiesel, which is produced from plant and animal-based feedstocks, represents an alternative to petroleum-based diesel fuel. It is essentially the bio-based analog for petroleum diesel that ethanol represents for petroleum gasoline. Chemically speaking, biodiesel is a mixture of mono-alkyl ester of fatty acids obtained from extracted plant oils and/or collected animal fats. Both plant oils and animal fats are commonly referred to as "lipid" feedstocks. Lipids are actually a class of chemicals found in plants and animals. This class of chemicals is relatively diverse in terms of chemical composition; however, the chemicals within this classification are categorized within this single common grouping due to their solubility within various organic solvents, such as benzene, ether, chloroform, and carbon tetrachloride (often known as "fat solvents"). Lipids are not very soluble in water. They are the primary energy storage system for animals, while plants utilize carbohydrates (starches) as their primary energy storage system. Chemicals classified as lipids include fats and oils, waxes, phosphoglycerides, sphinolipids, glycolipids, steroids, terpenes, prostaglandins, and fat-soluble vitamins. Among these chemicals, fats and oils are by far the most abundant lipids. Both are considered triglycerides because they are esters made up of three fatty acids joined to a glycerol molecule (which is a trioxy alcohol).

Most oils typically contain on the order of 14 fatty acids. If a quality feedstock is used, the bulk of these acids are bound as part of the triglycerides. Most, if not all, feedstocks contain free fatty acids, with the better feedstocks containing very few. The implications of containing high levels of free fatty acids are discussed in more detail later in this document; but, suffice it to state that their presence forms soap during production which poses processing challenges and is of little real value to most parties within the biodiesel industry (however, soap may be further processed into other 
chemicals that may be of value). As mentioned earlier, triglycerides can contain different types of fatty acids (thereby, they are mixed triglycerides). Fats tend to proportionally contain more saturated fatty acids and plants generally have more unsaturated fatty acids. If all three of the fatty acids are the same, then the triglyceride is known as a "simple" triglyceride. The plant oils commonly used in biodiesel production contain mostly oleic and linoleic acids. Most fats used or proposed for use in biodiesel production contain fairly equal portions of palmitic, stearic, and oleic acids as the dominant fatty acid composition.

Numerous processing techniques, both batch and continuous operations using either base or acid catalyzed reactions, have been tested and proven technically viable. Other techniques are being researched, including lipase induced reactions which were under development by the USDA in 2000. However, the base-catalyzed, methyltransesterification of soybean oil has been the predominant production technique used within the United States. During this process, the soy oil (previously extracted prior to production) is reacted with a primary alcohol (methanol $\left[\mathrm{CH}_{3} \mathrm{OH}\right]$ ) and a base (sodium hydroxide, aka. caustic $[\mathrm{NaOH}]$ ) to form the fatty acid mono alkyl ester (in this production case, a fatty acid methyl ester which is often referred to as FAME). This production reaction is summarized by the following reaction scheme:

Caustic

$$
\text { Triglyceride + Methanol } \rightarrow \text { Methyl Ester + Glycerol }
$$

This reaction is classed as the transesterification reaction. This reaction is similar to saponification, which is used to produce soap from fatty acids. On a weight balance basis for the transesterification reaction depicted above, for every 100 pounds of oil/fat and 10 pounds of methanol added, approximately 100 pounds of FAME and 10 pounds of glycerol are produced. This reaction reduces the molecular weight of the triglycerides by $60 \%$ when the methyl ester is formed. Additionally, viscosity is reduced eightfold and volatility increases.

The type of fatty acids and their respective concentrations found in triglycerides depends upon the feedstock source. Soybean oil is the main feedstock for the biodiesel produced in the United States, but in other parts of the world, different feedstocks are 
used. In South America, coconut oil is used to produce biodiesel, but in Europe, rapeseed oil is the primary feedstock. Soybean, coconut, and rapeseed oil are derived from vegetables, so climate heavily influences where these vegetables can be grown. Another feedstock is beef tallow, which is composed of the fat from cattle. The type and concentration of fatty acids in a feedstock are directly related to several key properties in the resulting biodiesel. The chemistry of the fatty acids are critical as to the quality of the final product - biodiesel. Therefore, the fatty acid composition of the feedstock plays a vital role in determining the feedstock's suitability for biodiesel production. Within the United States, soy oil is by far the most common feedstock which is not surprising when one considers that soy oil represents approximately $75 \%$ of the total poundage of plant oils produced in the United States on an annual basis. In descending order, the top agricultural plant oils produced on an annual basis within the United States are soy (18.3 billion Ibs.), corn (2.4 billion Ibs.), cotton seed (1.01 billion Ibs.), sunflower (1.0 billion Ibs.), and peanuts ( 0.22 billion lbs.). Commonly accepted biodiesel feedstocks include the oils from soy, canola, corn, rapeseed, and palm. New plant oils that are under consideration include mustard seed, peanut, sunflower, algae, and cotton seed.

In 2003 lowa State University estimated that if all oils and fat produced on an annual basis within the United States were used to produce biodiesel, this would represent only $15 \%$ of the total amount of petroleum diesel used on an annual basis within the United States. This is why the issue of finding new alternative feedstocks, such as fats, is essential to proving the ability of biodiesel to meet the energy needs of the United States. Hence, the project team decided to pursue the potential of developing novel lipid feedstocks for providing badly needed feedstock resources to the rapidly developing US biodiesel industry. The primary research direction focused on the utilization of lipids extracted from wastewater plant sludges. This potential feedstock is being produced at amazing annual tonnages, poses a disposal problem to many municipalities, is located near high diesel demand regions (cities), and is essentially free; hence, the research initiated was viewed as having a high potential to dramatically impact the future of bio-based diesels. 


\section{Overall Research Objective}

The overall objective of this effort was to perform research on the development of key process innovations on bio-based production techniques (and potentially other ligninase-based products) that match Mississippi's biomass resources while developing the existing and potential research capabilities of our cluster into a nationally recognized technical resource on the secondary production of fuels and other products from biomass. The effort was approached through a concurrent developmental initiative focusing on syngas fermentation, fermentation of acid hydrolyzates, enzymatic conversion of lignin, and production of biodiesel from novel feedstocks. This effort was to provide the technical foundation for enhancing the technical viability of chemical production from biomass via the performance of basic science, to develop the research capability within the university infrastructure in Mississippi, and to provide a bridge to existing industrial activities undertaken by some Consortium members for producing ethanol and other chemicals of industrial interest.

It is noteworthy to mention that the developmental effort undertaken by Consortium members fit directly into the State of Mississippi's current Science and Technology Plan which highlights biotechnology development as one of the four primary development goals for the state. Hence. these goals not only fit well with a rapidly developing research area which would better position Consortium members to compete nationally for funding, but it was also deemed of great importance to economic development thrusts underway within the State of Mississippi.

\section{Secondary Detailed Goals}

Several secondary developmental goals were developed by the project team working in concert with advisory board members - these goals are listed below by key topical area.

Fermentation of Syngas - The secondary goals for the fermentation of syngas research thrust were to:

1. Initiate a search for more efficient organisms (microorganisms).

2. Assess potential bacterial contaminants that may be established within a syngasfed fermenter. 
3. Optimize mass separation techniques for both the ethanol and the low levels of acetic acid produced as a by-product.

4. Evaluate the use of solid-substrate fermenters instead of the suspended growth units which have been used or proposed exclusively in previous work.

5. Evaluate methanogenic biotreatment of the distillation bottoms containing the acetic acid, potentially leading to the production of methane as a secondary energy source.

Acid Hydrolysis of Biomass to Produce Ethanol - Secondary objectives of the acid hydrolysis research thrust included:

1. Enhance conversion efficiency using improved acid impregnation, depolymerization, and extraction through development of innovative processing equipment designs and determination of the resulting production kinetics.

2. Evaluate how these process enhancements impact the formation of potential inhibitory by-products within the hydrolyzates that may adversely impact downstream fermentation.

3. Improve acid recovery using innovative recovery systems through the tailoring of new ionomeric membranes with controllable morphology-transport properties.

4. Develop a robust fermentation operation that is fully compatible to the extract solutions.

5. Performance of basic research to determine key kinetic and mechanistic information not generated by past work, which tended to focus more on broader design and economic information gathering.

Enzymatic Conversion of Lignin - Secondary objectives of this research thrust included:

1. Investigate lesser explored sources of lignin depolymerase activity, particularly those found in gut ecology.

2. Develop functional consortia of organisms for efficient depolymerization and disengagement of lignin from cellulose; determine the relation of redox conditions to the extent and nature of organism activity and generation of enzymes.

3. Develop functional assays for lignin degradation; use multiple levels of assays to screen candidate organisms.

4. Initiate process development using the best candidate agents and conditions, conduct bench-scale (5-gal bucket) pilot tests; apply molecular weight change models to characterize conversion of lignin, and determine rates.

Production of Biodiesel - Secondary objectives of this research thrust included:

1. Initiate process development via an evaluation of overall process feasibility.

2. Evaluation the performance of traditional lipid extraction for capturing lipids from biosolids. 
3. Determine if the lipids captured could be converted to a fatty methyl ester via traditional biodiesel processing.

4. Evaluate the potential costing savings using this novel process.

5. Develop relationships with key entities in the wastewater industry to ensure that a thorough understanding of the industry is established and used to guide research direction.

6. Vette the proposed process to biodiesel and fuels production industry experts to assess overall viability within an industrial setting. 


\section{SECTION II - CAPABILITY DEVELOPMENT WITHIN THE STATE OF MISSISSIPPI AS A RESULT OF THE ESPCoR FUNDING}

The funding support for this project came from the US Department of Energy's (DOE) Experimental Program to Stimulate Competitive Research (EPSCoR) program. The DOE EPSCoR program administered via the DOE's Office for Basic Science is very similar in scope and intent to most federal EPSCoR programs. The over-arching goal of EPSCoR programs, including the DOE program, is to increase the competitiveness of research units within states which historically have not obtained expected portions of federal developmental funding. Mississippi was identified as one of these states early in the development of the ESPCoR program within participating federal agencies.

The DOE program, in particular, emphasizes capability development from mainly a technical perspective. In short, it is an investment in science and research capability. This approach was an excellent fit for a group of university researchers from Mississippi working in concert with both state and federal policy makers in terms of positioning the State of Mississippi as a leader in the area of biofuels and other bioproducts development and the resulting commercialization. To a state like Mississippi with limited resources and little history as a "player" in this area, this type of effort was "the perfect fit."

This section presents a brief summary overview of the benefits gained by Mississippi from the funding by DOE EPSCoR for the program entitled "The Mississippi University Consortium for the Utilization of Biomass." It will present the position of Mississippi prior to the funding of this effort, then detail resulting gained benefits. This approach will enable the reader to better appreciate the value that the project provided, not only to the participating universities but to the entire state, and to appreciate as well the economic development gain experienced by Mississippi via the establishment of world class biofuels expertise within the state universities. 


\section{Development of Mississippi as a Leader in the Bio-Based Product Industry}

Historically, Mississippi has been a very agriculture oriented state in terms of all facets of societal observation. The comparatively small industrial base existing within the state is almost exclusively located in the coastal area. At the same time, some of the most economically depressed areas of the United States are located within the State of Mississippi; particularly within the Delta Region, where tremendous capability exists for crop growth along with current massive volumes of waste biomass. For any industrial base, including biofuels related technologies, to be developed within Mississippi it is essential that both a capable technology brain-trust and the research capability for supporting innovation and cutting-edge product development/marketing be established.

The project team used a three-pronged approach toward increasing both the research and industrial competitiveness of the State of Mississippi in the area of producing fuels and other chemicals/products from renewable bio-based feedstocks. The three areas used within this approach were Research Capability Development, Industrial Outreach, and Human Resource Development.

The following three chapters within are dedicated to summarizing the improved capabilities for each of the developmental areas mentioned above. These data are presented not only to highlight the great benefits that the EPSCOR funding provided the four participating universities in terms of increasing research capability and competitiveness; but also to exemplify how the State of Mississippi, in significant part due to this DOE EPSCoR project, was able to immerge as a developing leader within the United States as a state where both biomass to chemicals development and commercialization capability exists.

\section{Research Competitiveness and Capability Development}

Research Competitiveness and Capability are difficult parameters to accurately quantify because they encompass facilities, research inventiveness, grantsmanship, institutional support, quality of faculty, and a solid student pool. Hence, as the partner institutions of this program were designing the outline of this final report, it was decided that productivity in terms of peer-reviewed publications and new project funding obtained would serve as the indirect evidence of the increase in research 
competitiveness and capability because these products are the results of achieving the targeted improvement. The research summary section of this final report presents a summary of the many projects undertaken via the funding of this EPSCoR project.

\section{Peer-Reviewed Papers Published Via Work Derived from the Program}

The publication of peer-reviewed papers derived from work funded by the program was viewed by the project team as a key indicator of project success when viewed from a Human Research Development perspective. The project team used this criterion because through peer-reviewed publishing the faculty and students involved in the project became known experts in the biofuels arena. This recognition of expertise is a factor critical to success in securing competitive funding, involvement with policy making initiatives, and the interaction of peers from other institutions with program development.

The listing below represents selected papers published by the project team over the period of DOE funding for this project (plus one additional year):

\section{Mississippi State University}

1. Extraction of rice bran oil using supercritical carbon dioxide and propane. Sparks, D.; Hernandez, R.; Zappi, M.; Blackwell, D; and Fleming, T. Journal of the American Oil Chemist Society 2006. V83, pp. 885-891.

2. Development of a heterogeneous catalytic cracking reactor utilizing online mass spectrometry analysis. Benson, T.J.; Holmes, W.E.; White, M.G.; French, W.T.; Alley, E.G., and Hernandez, R. Journal of Chromatography A (0021-9673) 2007 Nov 23. Vol. 1172, Iss.2; p.204-8.

3. Reactions of fatty acids in superacid media: Identification of equilibrium products. Hernandez, Rafael; French, Todd; Alley, Earl; Holmes, William, and Benson, Tracy. Journal of Molecular Catalysis A: Chemistry (1381-1169) 9/3/2007. Vol. 274, Iss.1/2; p.173-178.

4. Solubility of azelaic acid in supercritical carbon doxide. Sparks, Darrell L.; Hernandez, Rafael; Estevez, L. Antonio; Meyer, Nicholas, and French, Todd. Journal of Chemical \& Engineering Data (0021-9568) 7/12/2007. Vol.52, Iss.4; p.1246-12496.

5. Feasibility of methanogenic digestion applied to a low $\mathrm{pH}$ acetic acid solution. Taconi, Katherine A.; Zappi, Mark E.; French, W. Todd, and Brown, Lewis R. Bioresource Technology (0960-8524) 5/15/2007. Vol.98, Iss.8; p.1579-1585.

6. Extraction of lipids from municipal wastewater plant microorganisms for production of biodiesel. Dufreche, S.; Hernandez, R.; French, T.; Sparks, D.; Zappi, M., and Alley, E.; J. Amer Oil Chem Soc. 2007, 84, 181-187. 
7. Development and comparisons of efficient gas-cultivation systems for anaerobic carbon monoxide utilizing microorganisms. French, W. Todd; Ford, Jack; Hernandez, Rafael; Easterling, Emily; Zappi, Mark; Morrison, Christine, and Brown, Lewis R. Bioresource Technology. 2008. 99, 638-643.

8. Determination of methanol in biodiesel by headspace solid phase microextraction. Paraschivescu, Maria C.; Alley, Earl G.; French, William T.; Hernandez, Rafael, and Armbrust, Kevin. Bioresource Technology. 2008. 99, 5901-5905.

9. Methanogenesis under acidic $\mathrm{pH}$ conditions in a semi-continuous reactor system. Taconi, Katherine A.; Zappi, Mark E.; French, W. Todd, and Brown, Lewis R. Bioresource Technology. 2008. 99, 8075-8081.

10. The effect of glycerol as a secondary substrate on the growth and fatty acid composition of Rhodotorula glutinis. Easterling, E. R.; French, W. T.; Hernandez, R., and Licha, M. Bioresource Technology. 2009. 100, 356-361.

11. Biodiesel production by in situ transesterification of municipal primary and secondary sludges. Mondala, A.; Liang, K.; Toghiani, H.; Hernandez, R., and French, T. Bioresource Technology. In Press.

\section{Jackson State University}

1. Enhancing ethanol fermentability of an artificial acid hydrolyzate with treatment of anion exchange resins. Zhang, Yi; Gao, Jun; Ntoni, Jennifer; Begonia, Maria F.T.; Lee, Ken S. and Hwang, Huey-Min. Canadian Journal of Microbiology. 2007.

2. Effects of selected by-products of an acid hydrolyzate on cell growth and ethanol fermentation by saccharomyces cerevisiae. Gao, Jun; Zhang, Yi; Ntoni, Jennifer; Begonia, Maria F.T.; Lee, Ken S.; Hicks, Lenore; Hwang, Wayne W., and Hwang, Huey-Min. Journal of Mississippi Academy of Sciences. 2006. 51(4): 220-230.

3. Effect of an acid hydrolyzate of southern pine softwood on the growth and fermentation ability of yeast saccharomyces cerevisiae. Zhang, Yi; Hwang, HueyMin; Begonia, Maria F.T.; Lee, Ken S., and Zeng, Kui. Journal of Mississippi Academy of Sciences. 2005. 50 (\#2): 138-143.

\section{University of Mississippi}

1. Determination of lignin by HPSEC using multi angle laser light scattering. Gidh, Aarti; Decker, Stephen; Vinzant, Todd; Himmel, Michael, and Williford, Clint. Journal of Chromatography A, 1114(1): 102-10. 2006.

2. Fungal-induced redistribution of kraft lignin molecular weight by multi angle laser light scattering. Gidh, Aarti; Decker, Stephen; Vinzant, Todd; Himmel, Michael, and Williford, Clint. Chemical Engineering Communication. 2006. Volume 193, Number 12, pp. 1546-1561(16).

3. Characterization of lignin using multi angle laser light scattering and atomic force microscopy. Aarti Gidh, Aarti; Decker, Stephen; See, Chun; Himmel, Michael, and Williford, Clint. Analytica Chimica Acta., (2006). 555 (2), p. 250. 
4. Cultures from wood-eating insect guts and waste-waters for lignin depolymerization screening. Gidh, Aarti; Talreja, Dinesh; Vinzant, Todd; Williford, Clint and Al Mikell. Applied Biochemistry and Biotechnology. 2006. Volume 131, Issue 1-3, pps. 829843.

\section{University of Southern Mississippi}

1. Mechanical and transport property modifications of perfluorosulfonate ionomer membranes prepared with mixed organic and inorganic counterions," Phillips, A.K., and Moore, R.B. J. Polym. Sci., Part B: Polym. Phys. 2006, 44(16), 2267. Special Issue on Polymers and Fuel Cells.

2. SAXS analysis of the thermal relaxation of anisotropic morphologies in oriented Nafion ${ }^{\circledR}$ membranes. Page, K.A.; Landis, F.A.; Phillips, A.K., and Moore, R.B. Macromolecules 2006, 39, 3939.

3. Ionic actuators based on novel sulfonated ethylene vinyl alcohol copolymer membranes. Phillips, A.K., and Moore, R.B. Polymer 2005, 46, 7788.

4. Influence of ionic aggregation on the surface energies of crystallites in poly (butylenes terephthalate) ionomers. Page, K.A.; Schilling, G.D., and Moore, R.B. Polymer 2004, 45(25), 8425.

5. State of understanding of nafion. Mauritz, K.A., and Moore, R.B. Chemical Reviews 2004, 104(10), 4535.

6. Photoinitiated grafting of maleic anhydride onto polypropylene. Pan, B.; Viswanathan, K.; Hoyle, C.E., and Moore, R.B. J. Polym. Sci., Part A: Polym. Chem. 2004, 42(8), 1953.

7. Alteration of membrane properties of perfluorosulfonate ionomers using solution and melt processing procedures. Phillips, A.; Bae, W.S.; Urban, M.W., and Moore, R.B. Polym. Prepr. (Am. Chem. Soc., Div. Polym. Chem.), 2005, 46(2), 578.

8. Sulfonated ethylene vinyl alcohol copolymers as electroactive active materials. Phillips, A.; Wolbert, M., and Moore, R.B. Proceeding of MACRO 2004, IUPAC, 2004.

9. Phase behavior in solution-processed perfluorosulfonate ionomers. Phillips, A.K., and Moore, R.B. Polym. Prepr. (Am. Chem. Soc., Div. Polym. Chem.), 2003, 44(1), 1142.

10. The effect of ion-incorporation on segmental orientation of polymer chains and guest/host interactions in oriented polymer/dye systems. Pan, B., and Moore, R.B. Polym. Prepr. (Am. Chem. Soc., Div. Polym. Chem.), 2002, 43(1), 358.

\section{Projects Obtained as a Result of Capability Enhancement}

EPSCoR's overarching goal is to increase the competitiveness of participating universities in obtaining research funding. A listing of projects approved at participating universities since DOE EPSCoR funding was received is presented below as evidence indicating the high level of success that the project achieved toward meeting this goal. It is interesting to note that before the funding of this project, Mississippi had enjoyed 
little to no funding in the biofuels area for a period of well over 20 years prior to the receipt of the DOE funds. Since then, the return on investment for DOE via R\&D funding brought into Mississippi was (at a minimum) a 4 to 1 return! This is a key area of pride for the project team. The following funded projects have been awarded since DOE EPSCoR funding was received:

Mississippi State University

1. Compatibility of Ethanol on Metallic Materials; $\$ 100,000$ over two years; funded by the FAA.

2. Microbially Enhanced Petroleum Recovery; $\$ 300,000$ over three years; funded by the DOE.

3. Development of Syngas Fermentation; $\$ 200,000$ over one year; funded by the DOE.

4. Commercialization of Syngas to Ethanol via Fermentation; $\$ 2,000,000$ over three years; funded by Mississippi Ethanol LLC.

5. Conversion of Poultry Litter to Biogas; $\$ 30,000$ over one year; funded by the Mississippi Technology Alliance

6. Development of a Bio-Based Commercial Adsorbent; $\$ 150,000$ over two years; funded by the USEPA

7. Development of Lipid Extraction from Rice Bran; $\$ 150,000$ over two years; funded by AgraPure LLC.

8. Drafting of the Mississippi Biodiesel Engineering Assessment Manual; $\$ 30,000$ for one year; funded by the Mississippi Technology Alliance.

9. Evaluation of Digestion Methods for Production of Biogas from Manure-Based Feeds; $\$ 35,000$ for two years; funded by Mississippi Technology Alliance.

10. Establishment of a Regional CHP Center; $\$ 2,500,000$ over five years; funded by the US DOE.

11. Development of Syngas to Fuels; $\$ 3,000,000$ over five years; funded by the USDA.

12. Biodiesel Development; $\$ 2,000,000$ over three years; funded by the DOE.

13. Biodiesel from Sewage Sludge; $\$ 200,000$ over three years; funded by the USEPA.

14. Biofuels Development; $\$ 1,000,000$ over two years; funded by the DOE.

15. Sustainable Energy Research Center, $\$ 5,000,000$ over three years; funded by DOE.

\section{Jackson State University}

1. Structural Basis for the Chloroperoxidase-Catalyzed Enantioselective Transformations, Dr. Xiaotang Wang, NSF/Career (2003), funded, total support requested: $\$ 649,370 / 5$ years. 
2. Depolymerization of Lignocellulose by Fungal Cells and Immobilized Enzymes, DOE-MRT/SBIR subcontract project from University of Mississippi. 8/1/06-7/31/07, $\$ 225,000$ for 1 year.

\section{University of Southern Mississippi}

1. Studies of PEMFC Membrane Durability. E. I. DuPont de Nemours \& Co., 10/01/03 to $09 / 30 / 06, \$ 250,000$.

2. Morphological Factors Affecting the Transport Properties and PEMFC Performance in Nafion ${ }^{\circledR}$-Based Membrane Electrode Assemblies. Nissan Motor Co., Japan, 4/1/06 to $6 / 30 / 07, \$ 107,732$.

3. NSF MRSEC-Center for Response Driven Polymeric Films (Moore Component: Ionomeric Polymer-Metal Composites for Artificial Muscles and Sensors). NSF, MRSEC, $10 / 1 / 02$ to $9 / 30 / 08, \$ 37,000 / y r$.

4. Improved Membrane Materials for PEM Fuel Cell Applications. DOE, 6/1/06 to 11/31/07, \$618,750.

5. Manipulation of Aggregate Organization in Ionic Polymers to Elucidate and Enhance Morphology-Actuation Relationships in Nanostructured Electroactive Polymer Systems. NSF (ANN-NER), 8/1/07 to 7/31/08, \$115,000.

\section{University of Mississippi}

1. Mississippi Biomass Utilization: University-Based Research and Development Project, funded by U.S.DOE through The Mississippi Technology Alliance-Strategic Biomass Initiative. A project under The Mississippi Research Consortium by the four comprehensive universities of The State of Mississippi aiding commercialization of renewable, biomass-based fuels and chemicals. Amount: $\$ 900,000$. Period of performance: August 2006-July 2007.

\section{Other Research Competitiveness and Capability Improvements}

Faculty Active in Bio-Based Product Development Population Dynamics - Based on a survey of the four participating universities it is estimated that on average the number of faculty involved in biomass to chemicals related research appears to have increased by two due to the stimulation of interest in this area generated by the project. In fact, most of the participating universities initiated cluster hiring of faculty having expertise in renewable energy due in a large part to the research interest initiated by this project.

Technical Presentations - The number technical presentations made at regional, national, and international conferences and symposia are not listed within this chapter. However, a conservative estimate of the total number of presentations made which presented all or part of work performed under funding from this project is in excess of 
100 presentations made over the six years of DOE funding. It is noteworthy to mention that since the funding has ended, presentations in this topical area have increased in terms of numbers per research team indicating continued activity within the renewable energy area (which is the key goal of EPSCoR).

\section{Economic Development/Industrial Assistance}

The partners associated with the Consortium have been very active with the positioning of Mississippi as a leader within the alternative energy arena since the funding of the EPSCoR project. Efforts supporting this goal include (1) direct technical assistance to industries to improve the performance of their designs and/or existing facilities, (2) collaboration with industries and other private entities to secure both state and federal funding, (3) evaluation of potential process operations via laboratory assessments, assisting with the recruitment of industries into the state, and (5) presentation of results at industry planning meetings. A conservative estimate is that over 50 companies and 10 governmental agencies have interacted with Consortium partners to stimulate economic development within the State of Mississippi.

\section{Human Resources Development}

The burden of developing high technology-based industries in states without an existing technologically trained labor base falls almost exclusively on the shoulders of the state's university system. However, universities cannot train future technologists without students wanting to enter these fields. Therefore, Human Resource Development (HRD) is the key to success. The HRD program within the Mississippi University Research Consortium for Utilization of Biomass features student involvement as its cornerstone. Over 50 students have participated within this program over the past 2.5 years. Of these, approximately $50 \%$ are classified as being from under-represented minority groups. Additionally, over 30 presentations were given by students at various meetings. Several awards were won by our students participating in student paper/poster contests. Consortium students and faculty members made numerous presentations at local community colleges and various universities to student and faculty groups. Presentations were also given by visiting DOE officials and TAC members. Clearly, although not directly funded under this program, a significant effort 
has been made over the past few years to expand the scientific and engineering student base within Mississippi.

The team was advised upon notification of project funding by DOE in 1999 that human resource development (HRD) activities were not to be directly funded under this program. Rather, the team was asked to integrate HRD as an underlying activity within the research program. As a result of this request, an aggressive Human Resources Development (HRD) Plan was developed to ensure that the proposed project had farreaching impact on the development of a biofuels and other bio-based products educational base within the State of Mississippi. The approach of integrating human resource development and research used over the course of the program has proved to be successful based on student interest and subsequent involvement in the research program.

As stated previously, Mississippi has historically been a very agriculturally oriented state. A comparatively small industrial base exists within the state, which is concentrated almost exclusively in the coastal area. The State of Mississippi contains some of the most economically depressed areas of the United States. Recent US Census Bureau estimates place over $20 \%$ of Mississippians below the poverty level. Essentially, this means that many of the students within the State of Mississippi have very limited direct exposure to the observable benefits that technology fields offer due to the limited industrial presence within their communities. Of particular concern are the small percentage of minorities and females (herein referred to as "under-represented" groups) entering technology majors at Mississippi universities. For any industrial base, including fuels related technologies, to be developed within a state a capable technology brain-trust must be built and a research capability must be in-place to support innovation and cutting-edge product development/marketing. The production and development of alternative fuels, which is the future of all developed societies, requires a steady flow of properly trained technologists and the development of economically competitive fuel production techniques. Therefore, Human Resource Development is the key to success.

Of particular interest to the State of Mississippi and its fuels-technology future is the fact that since Mississippi is a state whose economy is based primarily on 
agriculture and forest products industries, a high volume of biomass is currently being wasted which could be utilized for the production of alternative fuels. Mississippi is in a very unique position to become a leader in primary alcohol production from biomass and the home to a wealth of supporting technicians. For Mississippi to achieve its leadership potential, not only must cost-effective technology that can produce alternative fuels be made available, but a sufficient base of technologists must be established to meet the projected demands of this industrial growth. Clearly, increasing the number of students entering and graduating in technology-based fields will accelerate the development of a viable alternative fuels program and, at the same time, increase the economic competitiveness of the State of Mississippi. Finally, the opportunities afforded minority students finishing in a technology-based field offer a basis for the development of a system of role models for under-represented groups.

Overall, the HRD philosophy of the Consortium for increasing the flow of qualified students through the technology education pipeline was manifested through a two pronged approach. First, an aggressive recruitment effort focusing on university students at both the undergraduate and graduate levels was implemented. Interaction using both indirect (websites and teacher packets) and direct (student employment opportunities) venues was used to reach a wide audience while directly targeting the most promising students that have a high potential for completing the challenging curriculums of technology fields.

The second facet of the HRD philosophy is retention of promising students within the technology fields, once they have entered those programs. Programs such as research internships and close interactions with their peers within their own institution and the other member universities are critical to retaining these students. Although, the proposed HRD will not exclusively focus on under-represented minorities, they will be a particular target because of the population dynamics of these groups in Mississippi and the need for a higher participation more reflective of the state's demographics.

\section{Objectives of the HRD Efforts}

The following objectives have been identified to facilitate increasing the flow of qualified technologists through the education pipeline: 
1. Set the hiring of under-represented groups for undergraduate and graduate student positions as a top priority.

2. Maximize the use of funding toward student hiring.

3. Expose college students to energy related issues via presentations at numerous universities and community colleges.

4. Focus on student presentations at Consortium meetings and technical society meetings to develop their presentation skills.

Overview of Consortium HRD Activities

This subsection will present a brief description of major HRD activities undertaken by the Consortium.

\section{- Consortium Meetings}

As part of the Program Management Plan, the Consortium members met three times a year at times corresponding to the end of each academic semester. This effort fostered increased collaboration and camaraderie among the project team members. The primary purpose of these meetings was technology exchange between the research team members; however, the meeting also provided an excellent opportunity for HRD activities. At least two technical presentations were made to technology-based classes at the host institution during these meetings. Bringing the Technical Advisory Committee (TAC) members and DOE Program Managers to each Consortium institution also enhanced exposure of the Consortium members and their students to key personnel within the field. Additionally, DOE personnel and TAC members were solicited to make presentations to the faculty and graduate students at the host institution during the TAC reviews.

\section{- Consortium Internet Website}

A website for the Consortium was operated by MSU which featured the Consortium structure, key contacts, its technical mission, staff capability, current project summaries, and an overall description of the DOE EPSCoR program. The site also linked other websites of interest (i.e. DOE sites, including the DOE-ESPCoR site. Many comments and inquiries were received over the seven year period of site operation. 


\section{- DOE and Other Federal Facility Visits}

A series of visits to pertinent federal laboratories, including DOE and USDA laboratories and offices, were initiated at a targeted rate of at least one 2 day visit per member institution per year. Members were encouraged to include their students on these visits. These visits provided at minimal an opportunity to brief the laboratory staff on current Consortium activities (particularly those of the visiting scientist), while allowing the visitor to learn more about the activities of the host laboratory. Longer visits were possible using a variety of summer programs offered by many of the federal laboratories (ORNL offers a one-time funding source for OREU universities to visit their laboratory).

\section{- Consortium Researcher/Student/Equipment Exchange}

All Consortium members agreed to open their facilities for visits from researchers and/or students from the other Consortium members for on-site technology exchange. This interaction served to enhance the total research capability of the Consortium and foster a strong teaming spirit, while at the same time stimulating further student interest in the fuels related area. JSU agreed to work with MSU on teaching developing phospholipid fatty acid GC-based techniques to interested MSU scientists. Conversely, MSU worked with JSU to design fermentation experiments and provide techniques for ethanol analysis. Additionally, USM agreed to loan JSU a large laboratory fermenter for performance of some of their fermentation experiments. Clearly, these interactions were very positive and strengthened the long-term stability of the Consortium, while allowing for increased interaction between researchers and students.

\section{- Student Involvement}

Graduate Students - All of the projects proposed in the R\&D Effort of this proposal involved the provision of graduate student opportunities at each member institution. Particular emphasis was placed on the selection of students from under-represented groups. This opportunity, albeit the most time and funds intensive outreach activity, offered the highest potential for producing fuel technology oriented professionals. Over 20 graduate students were supported by this effort. A unique education opportunity was developed to increase the quality of the technologists produced 
from this activity. All of the students working on each technology thrust area of the $R \& D$ cluster were required to interact with colleagues from the other universities contributing to the thrust area. On several occasions, the students prepared joint presentations. This activity stimulated the building of teaming skills and increased the interest level of each student toward the overall subject area. These students have been and will continue to be active in making presentations to student groups at various educational levels (i.e. community college, high school, and elementary school). As one means for evaluating the long-term success of this program, all students which graduate from this program will be tracked over the course of their careers to assess the long-term results of this effort.

Undergraduate Students - The hiring of undergraduate students was found to be an excellent recruitment tool for filling future graduate student positions. Each university had a suite of undergraduate student positions available over the course of each three year performance period. The top seniors and/or juniors from each of the member institutions were heavily recruited. Success for this effort was gauged based on the number of students entering fuel-related jobs or the number pursuing advanced degrees. Thus far, numerous students from this program have indeed elected to go on to pursue higher degrees within the energy related area. This is indeed indicative of a very successful HRD effort and a source of pride for the Consortium members.

Students Involved in the Program - The following list presents students who have been a part of Consortium research activities. As can be seen from this extensive list, a significant number of students have been reached via inclusion within the R\&D program. Over 60 students have been a part of this project; of these, 28 are from under-represented minority groups. Also, several of the students who have been involved in this project elected to proceed to the next educational level and remain active with the project (i.e. MS graduates and remained active on the project to receive a $\mathrm{PhD}$ ). Others elected to go on to the next education level, but at nonmember institutions. This is another indication as to the very positive benefits this project has provided Mississippi in terms of expanding its science and technology 
base. It is interesting to note that almost $60 \%$ of the students electing to go on to a higher educational level are from under-represented minority groups.

University

MSU
Personnel

Mr. Allen Tieling Xie

Ms. Katherine Taconi

Mr. Rafael Hernandez

Ms. Holly Martin

Mr. Stephen Dufreche

Mr. Darryl Sparks

Mr. Tracy Benson

Ms. Veronica Locket

Mr. Ben Hartenbower

Mr. Douglas Tolar

Mr. David Neaves

Mr. Jack Ford

Ms. Christine Morrison

Ms. Maxine Jones

Ms. Alissa Willis

Mr. Britton Eyles

Ms. Beth Hester

Ms. Katrina Parker

Mr. Michael Begonia

Mr. John Sorrell

Ms. Shamika Brooks

Mr. Jason Black

Ms. Neena Joshi

Ms. Jessica Webb

JSU
Ms. Jennifer Ntoni

Ms. Ana L. Balarezo

Ms. Natasha Palmer

Ms. Valarie O'Bannon

Ms. Afrachanna Butler

Ms. Juanquina Thomas

Ms. KaShiris Walton

Ms. Rose Mugogo

Ms. Tyronza Lee

Ms. Baoying Zheng

Mr. Nagi Reddy

Ms. Lenore S. Hicks

Mr. Oscar Hundley

Ms Valarie O'Bannon

Mr. Vijay Ezkiel

Mr. Vidya Janga

Ms. Talia Sanders
Department/Position

Chemical Engineering/PhD Student Chemical Engineering/PhD Student Chemical Engineering/PhD Student Chemical Engineering/PhD Student Chemical Engineering/PhD Student Chemical Engineering/PhD Student Chemical Engineering/MS/PhD Student Chemical Engineering/MS Student Chemical Engineering/MS Student Chemical Engineering/MS Student Chemical Engineering/MS Student Chemical Engineering/MS Student Chemical Engineering/MS Student Chemical Engineering/UG Student Chemical Engineering/UG Student Chemical Engineering/UG Student Chemical Engineering/UG Student Chemical Engineering/UG Student Biological Sciences/MS Student Biological Sciences/MS Student Biological Sciences/UG Student Biochemistry/UG Student

Biochemistry/UG Student Biochemistry/US Student

Environmental Sciences/PhD Student Biological Sciences/MS Student Biological Sciences/MS Student Environmental Sciences/MS Student Biological Sciences/MS Student Biology/MS Student Biology/MS Student Biology/MS Student Biology/MS Student Environmental Science/MS Student Environmental Science/MS Student Chemistry/MS Student

Biology/MS Student Environmental Science/MS Student Environmental Science/MS Student Computer Science/MS Student Environmental Science/MS Student 
$\underline{\text { University }}$

JSU (continued)

UM

USM

\section{Personnel}

Ms. Tracy Ross

Ms. Erica Dillon

Ms. Veleka N. Jones

Ms. Courtney Young

Ms. Katrina Dunn

Ms. Centeria Cornellius

Ms. Sharon Hurley

Ms. Delphine Woody

Mr. Jeffrey Thorn

Ms. Ashley White

Ms. Teresa Demeritte

Mr. Antonio Woods

Ms. Shantelle I. Hughes

Mr. Lennis Jones

Ms. Esmeralda Fuentes

Mr. Omar Nava

Mr. Dinesh Talreja

Ms. Aarti Gidh

Mr. Sulabh Koolwal

Mr. Marty Lanier

Mr. Naresh Budhavaram

Mr. Avery Mixon

Ms. Jessica Doss

Mr. Eric Taylor

Mr. Alan Phillips

Ms. Angels Domenech

Mr. Bo Pan

Mr. Lane Windham

Mr. Wing Lian

Ms. Anika Geoghagan

Mr. John Rushing

Mr. Thomas Vincent

Mr. Chris Lewis

Mr. Micheal Wolbert

Ms. Lea Locke

Mr. Sean Bellman

Ms. Moonhee Lee

Mr. Graham Behringer

Mr. Jeremy Miller

Ms. Erica Colby

Mr. Mark Vincint

Ms. Bonnie Cook

\section{Department/Position}

Biology/MS Student

Visiting Med Student

Biological Sciences/UG Student

Biological Sciences/UG Student

Biological Sciences/UG Student

Chemistry/UG Student

Chemistry/UG Student

Chemistry/UG Student

Chemistry/UG Student

Chemistry/UG Student

Chemistry/UG Student

Chemistry/UG Student

Chemistry/UG Student

Visiting High School Student

Visiting High School Student

Visiting High School Student

Biology/PhD Student

Chemical Engineering/MS/PhD Student

Chemical Engineering/MS Student

Chemical Engineering/MS Student

Chemical Engineering/MS Student

Chemical Engineering/UG Student

Chemical Engineering/UG Student

Polymer Sciences/PhD Student

Polymer Sciences/PhD Student

Polymer Sciences/PhD Student

Polymer Sciences/PhD Student

Polymer Sciences/MS Student

Polymer Sciences/UG Student

Polymer Sciences/UG Student

Polymer Sciences/UG Student

Polymer Sciences/UG Student

Polymer Sciences/UG Student

Polymer Sciences/UG Student

Polymer Sciences/UG Student

Polymer Sciences/UG Student

Polymer Sciences/UG Student

Polymer Sciences/UG Student

Polymer Sciences/UG Student

Polymer Sciences/UG Student

Polymer Sciences/UG Student

Polymer Sciences/UG Student 


\section{Development of Relationships with Key Entities}

To facilitate the development of relationships with key entities within the biomass to energy field and to ensure that the technical projects undertaken by the Consortium members, a Technical Advisory Committee (TAC) was formed. These members interacted via a wide variety of methods over the seven year period of funding. Their involvement was deemed highly-valuable by the project members. The key membership of the TAC including a summary of their primary interactions is detailed below:

\section{Entity}

DoD USAE Waterways Experiment Station

Stennis Space Center

DOE National Renewable Energy Laboratory (NREL)

DOE Oak Ridge National

Laboratory (ORNL)

DOE Argonne National Laboratory (ANL)

Tennessee Valley Authority (TVA)

Mississippi Ethanol (ME)

Mississippi Development Authority

Headquarters, DOE

Mississippi Alternative Energy Enterprises $\underline{\text { Role }}$

Provided Training Opportunities to Consortium Members and Membership on TAC

Provided Training Opportunities to Consortium Members

Provided Training Opportunities to Consortium Members and Membership on TAC

Provided Training Opportunities to Consortium Members and Membership on TAC

Provided Training Opportunities to Consortium Members and Membership on TAC

Provided Training Opportunities to Consortium Members and Membership on TAC

Membership on TAC

Membership on TAC

Membership on TAC

Membership on TAC 


\section{SECTION III - PROJECT SUMMARIES \\ INTRODUCTION}

This section presents a series of summary papers drafted to highlight several projects undertaken by the project team over the course of the project. Each paper is presented by topical area of research along with the lead research entity of the Consortium that performed the experimental testing. Note that these papers are selected as feature efforts; however several other research initiatives were performed. The project team selected these papers as examples to highlight the diversity of both topics and levels of development that the project undertook. 


\section{DEVELOPMENT OF NOVEL BIOCATALYSTS \\ Performer: Mississippi State University \\ INTRODUCTION}

The overall objective of this research effort was to determine if carbon monoxide could be economically converted to ethanol microbiologically. While it is a wellestablished fact that microorganisms can convert carbon monoxide to ethanol, the question arises as to whether this can be achieved economically. Calculations, based on literature reports, leave serious doubt as to the economic viability of the process. Some of the factors effecting the economics of the process include (1) the rate of ethanol production by the microorganism, (2) the cost of media employed, (3) types and amounts of waste products from the microorganism that require treatment before discharge, (4) factors affecting the recovery of alcohol, and (5) characteristics of the microorganism employed, particularly its genetic stability and survivability in the presence of oxygen in air.

This report will summarize the results achieved in attempting to meet the above objectives. Specifically, these efforts involved working with two cultures purchased from the American Type Culture Collection (ATCC), isolation of cultures producing ethanol from carbon monoxide, determining the effect of alternation in the composition of the medium, gas mixture, and growth conditions on the production of ethanol, testing the feasibility of utilizing immobilized enzyme technology to produce ethanol from syngas, and studies on microbial growth and ethanol production from syngas at $90^{\circ} \mathrm{C}$ (hyperthermophiles).

\section{MATERIALS AND METHODS}

Most of the work was conducted in $60 \mathrm{ml}$ glass vessels fitted with mininert valves as shown in Figure 1 or $70 \mathrm{ml}$ Wheaton serum vials closed with butyl rubber stoppers and sealed with aluminum caps (Figure 2).

The medium employed for the ATCC cultures is given in Table 1 and the medium employed for all cultures was ATCC Growth Medium 1754 and is given in Table 2. 
Pure carbon monoxide was obtained from NexAir and the syngas was obtained from AirGas and consisted of 15\% carbon dioxide, 15\% methane, $30 \%$ carbon monoxide, and $40 \%$ hydrogen.

Gas analyses were performed on a Fisher Gas Partitioner Model 1200 (dual column, dual detector chromatograph). Column 1 was a $20 \mathrm{ft} \times 1 / 8$ in aluminum column

packed with $37.5 \%$ DC-200/500 on 80/100 mesh chromosorb P-AW and column 2 was a $6 \mathrm{ft} \times 3 / 16$ in aluminum column packed with 60/80 mesh molecular sieve, 13X. The column temperature was $70^{\circ} \mathrm{C}$ and the injector temperature was $65^{\circ} \mathrm{C}$. Helium gas was employed as the carrier gas at a flow rate of $35 \mathrm{ml}$ per min.

Ethanol analyses were performed using an Alcohol Analyzer AM1 (Analox Instruments, Lunenburg, MA).

\section{RESULTS}

\section{Work with ATCC Cultures}

Neither C. acetobutylicum (ATCC No. 824) nor C. ljungdhalii (ATCC No. 55383) would produce ethanol using Modified ATTC Growth medium (hereinafter referred to as MGM) even in the presence of carbon monoxide. Apparently, the organisms preferentially used a carbon source other than carbon monoxide. Resting cell suspensions of both organisms did produce ethanol from carbon monoxide in Ethanol Production Medium (herein referred to as EPM). The C. acetobutylicum culture was found to be contaminated with a gram-positive coccus that produced more ethanol than either of the purchased cultures.

\section{Isolation of Mesophilic Carbon Monoxide-Utilizing Cultures}

Isolation of CO-utilizing cultures was carried out using sewage sludge from a digester and sludge from a hog waste lagoon. A total of 21 media was employed for the isolation of cultures.

The Basal Mineral Solution (BMS) consisted of $\left(\mathrm{NH}_{4}\right)_{2} \mathrm{SO}_{4}, 50 \mathrm{mg} ; \mathrm{NH}_{4} \mathrm{Cl}, 50 \mathrm{mg}$; $\mathrm{KH}_{2} \mathrm{PO}_{4}, 68 \mathrm{mg}$ in $100 \mathrm{ml}$ distilled water. Various supplements were added including a vitamin solution, a trace element solution, brilliant green, oxgall, and azide. Rila sea salts were employed as a replacement for the trace element solution in some media since previous work had shown that seawater from the Pacific Ocean could replace the 
trace element solution and thus cut medium costs. Brilliant green and oxgall inhibit gram-positive microorganisms and sodium azide inhibits gram-negative microorganisms. Marine Broth was substituted for the production medium in some cases. The composition of the 21 media is given in Table 3 . All cultures were incubated under an atmosphere containing carbon monoxide at $30^{\circ} \mathrm{C}$ under either shake or static conditions.

Unexpectedly, most of the enrichments from both inocula produced ethanol. In order to gain some insight into the rate of ethanol production by the cultures, it was decided to calculate the rate over the entire 72-hour period of incubation. It is realized that the rate over this period of time would be far less than the maximum rate calculated during the logarithmic phase of growth. Even so, the rate of ethanol production over the 72-hour period was significant, even when compared to the fastest rate reported in the literature $(0.225 \mathrm{~g}$ ethanol $/ \mathrm{g}$ cells $/ \mathrm{hr})$ that was calculated for cells in the logarithmic phase of growth. As shown in Figure 3, enrichments from the hog waste lagoon sludge incubated under shake conditions produced as much as five times more ethanol than cultures incubated under static conditions. Transfers of the cultures were made and it was observed that in some of the cultures the rate of ethanol production was somewhat greater after $24 \mathrm{hrs}$ than it was after $48 \mathrm{hrs}$, suggesting that either some of the ethanol was being consumed by the ethanol producer itself or by contaminating organisms in the enrichment. Representative data are shown in Figure 4. When transferred into new media, ethanol production increased, but after a second transfer the rate of ethanol production in all cultures was less. Representative data are shown in Figure 5. Once again these data suggest that either the ethanol-producing cultures were using the alcohol they had produced or contaminating cultures that consumed ethanol were increasing in number.

As stated previously, oxgall and, to some extent, brilliant green either prevented or reduced the amount of ethanol produced from the carbon monoxide. These findings suggest that the microorganisms responsible for the conversion of carbon monoxide to ethanol are gram positive. Furthermore, as reported in the literature, some grampositive, spore-forming anaerobes convert carbon monoxide to ethanol. To determine if it is gram-positive, spore-forming anaerobes in the sludge from hog waste which are 
responsible for the production of ethanol from carbon monoxide, the following experiment was conducted. One set of vials, containing $10 \mathrm{ml}$ of medium was inoculated with one $\mathrm{ml}$ of sludge from the hog waste lagoon while another set of vials was inoculated with one $\mathrm{ml}$ of sludge after exposing it to $80^{\circ} \mathrm{C}$ for $10 \mathrm{~min}$ to destroy vegetative cells. Each set of vials contained the different media listed in Table IV except media 18-21 that were prepared using Marine Broth instead of Basal Mineral Solution. One set of the vials was incubated under static conditions while the other set was incubated under shake conditions. All vials had an atmosphere containing carbon monoxide. Incubation was carried out at $30^{\circ} \mathrm{C}$.

After 12 days of incubation, the following results were obtained. None of the spore systems containing oxgall or brilliant green used carbon monoxide or produced ethanol. Furthermore, none of the unheated cultures containing oxgall used carbon monoxide or produced ethanol while the unheated cultures containing brilliant green did use carbon monoxide and produced ethanol. Apparently, the oxgall at the concentration employed in the media was a more effective inhibitor of gram-positive microorganisms than was the brilliant green at the concentration employed. Of course, in the unheated cultures there should have been much larger numbers of gram-positive microorganisms since they contained both vegetative cells and spores.

The fact that sodium azide did not inhibit carbon monoxide utilization, ethanol production, or carbon dioxide production, further confirms the fact that the ethanolproducing microorganisms are gram positive (see Figures 6-8).

Efforts were made to obtain pure culture isolates from the more promising enrichment cultures. Solidified MGM, contained in plastic Petri plates were streaked and incubated anaerobically at $30^{\circ} \mathrm{C}$. After 5-7 days of incubation, small, well-isolated colonies were visible (see Figure 9). Some of these colonies were picked into culture vessels containing the MGM and were incubated anaerobically under shake conditions at $30^{\circ} \mathrm{C}$. Representative ethanol production from carbon monoxide by the isolates is given in Table 5. A photomicrograph of the cultures is shown in Figure 10.

In terms of ethanol production, the culture produced $15.4 \mathrm{mg} / \mathrm{DL}$ in $4 \mathrm{hrs}$ at $30^{\circ} \mathrm{C}$ using cells collected by centrifugation and resuspended in production medium with syngas. This was the only culture tested for alcohol production because of the 
problems associated with maintaining viable cultures. The test was merely to verify that the culture would produce ethanol from syngas as well as carbon monoxide alone. No attempt was made to optimize the alcohol production by this culture at the time. The most active isolate was selected to determine if growth and/or ethanol production could be enhanced by modifying the composition of the medium or changing the conditions of incubation. As a result of these studies it was found that:

1. the addition of boron, cobalt, iron, molybdenum, or nickel did not enhance growth or ethanol production,

2. neither fructose, glucose, glycerin, nor peptone enhanced growth or ethanol production,

3. alteration of the concentration of nutrients in the growth medium (concentrations of $1 / 16,1 / 8,1 / 4,1 / 2$, and 1 ) did not result in any change in growth and/or ethanol production. [This suggests that lesser amounts of nutrients can be employed which could reduce costs],

4. growth and/or ethanol production in filter-sterilized spent medium was unchanged,

5. growth and/or ethanol production was best in a medium of $\mathrm{pH} 7.0$ rather than $4.0,5.0,6.0$, or 8.0 ,

6. the isolate would not grow in the medium alone or with $2 \%, 4 \%$, or $6 \%$ ethanol added,

7. growth and/or ethanol production was better at $30^{\circ} \mathrm{C}$ than it was at $25^{\circ} \mathrm{C}$, $37^{\circ} \mathrm{C}$, or $55^{\circ} \mathrm{C}$,

8. growth and/or ethanol production was better at lower concentrations of carbon monoxide in the atmosphere when tested at $8 \%, 25 \%, 33 \%, 42 \%$,or $100 \%$ carbon monoxide,

9. neither magnetism nor light had any impact on growth and/or ethanol production,

10. a ratio of $20 \mathrm{Na}: 1 \mathrm{~K}$ in the medium yielded better growth than did $\mathrm{Na}: \mathrm{K}$ ratio of $100 \mathrm{Na}, 100 \mathrm{~K}$, or $20 \mathrm{~K}: 1 \mathrm{Na}$,

11. the addition of cysteine, cystine, glutathione or thioglycolate did not enhance growth while oxyrase and pyrite helped but alabandite was the best as shown in Figure 11. [This suggests that the oxidation-reduction potential of the medium was a significant factor in the growth of the isolate. Unfortunately, our supplier of alabandite ceased selling alabandite, and a new supplier has not been found. Studies using a gradient plate methodology and $\mathrm{Na}_{2} \mathrm{~S}$ as the reductant seemed to suggest that a heavy concentration of $\mathrm{Na}_{2} \mathrm{~S}$ was inhibitory to growth.],

12. The preference of the culture to grow on something rather than suspended in a medium is clearly shown in Figure 12. 
In addition to sewage sludge and sludge from a hog waste lagoon, enrichments made from an anaerobic treatment lagoon, animal manure, swamp sediment, carcass processing wastewater, and coal samples demonstrated the production of ethanol from carbon monoxide.

In considering the problem of increasing cell yield, it is known that most microorganisms prefer to grow on a solid medium rather than in a liquid medium. Furthermore, a much greater density of cells can be obtained by growth on solid media. Additionally, harvesting cells from a solid medium eliminates the need to concentrate cells by centrifugation or filtration, an important consideration from an economic standpoint. There are, however, several drawbacks to growing cells on solid media. For example, solid culturing techniques are difficult or impossible to scale-up and the cells may not be physiologically homogenous. Using a biphasic culturing technique these drawbacks can be obviated. Simply stated, the technique uses a thin layer of a liquid overlying a solidified nutrient medium. In fact, in some cases, water can be the overlying liquid provided that all of the necessary nutrients are present in the solid medium. Therefore, the first approach to increasing cell yield was to explore the possibility of using this biphasic growth technique. For this work a Rocking Platform (Model 200, from VWR) was purchased and placed in a Coy Glove Bag (Coy Laboratory Product, Inc.). Approximately $0.5 \mathrm{ml}$ of an enrichment culture growing in MGM was spread evenly onto the surface of solidified MGM contained in sterile 15x100 $\mathrm{mm}$ plastic Petri plates. Five $\mathrm{ml}$ of the liquid MGM was added to each plate; the plates were placed on the Rocking Platform machine and incubated. Heavy growth was observed on numerous plates and, when the cells were suspended in the EPM with a carbon monoxide atmosphere, ethanol was produced. Follow-up experiments yielded heavy growth but it appeared that contaminants had taken over since the cell suspensions no longer produced ethanol from the carbon monoxide. This technology holds considerable promise in the current project since large numbers of cells are required for both a suspended type of fermentation process and an immobilized cell type of process. It is interesting to note that cell concentrations as high as $10^{11}$ have been obtained using this technique which is 100 to 1000 times that normally produced 
in liquid cultures. If successful, this technology could drastically reduce the cost of the ethanol-producing process.

The second approach to increasing cell yield involves the use of alabandite $\left(\mathrm{MnS}_{2}\right)$ and pyrite $\left(\mathrm{FeS}_{2}\right)$ in liquid cultures. It was speculated that either and/or both of these materials could help in the reduction of carbon monoxide to ethanol and also serve as a site for cell attachment and growth. Therefore, small chips of either alabandite or pyrite were added to some of the enrichment cultures in MGM from which pure cultures were being sought. Incubation was at $30^{\circ} \mathrm{C}$ in closed vials under static conditions in an atmosphere containing carbon monoxide. Several of the cultures showed heavy growth on the alabandite chips as illustrated in Figure 11. Pyrite was colonized also but to a lesser extent that the alabandite.

\section{Evaluation of Immobilized Cell Technology}

Rather than employ a conventional suspended culture type fermentation, an immobilized cell type of scenario might be advantageous because it can (1) lower the cost of the suspending medium, (2) offer the potential of doubling the reaction rate by increasing the temperature $10^{\circ} \mathrm{C}$ (cell growth does not take place in this system), (3) lower the problems associated with contaminating organisms, (4) reduce the kinds and amounts of waste products requiring treatment, and (5) facilitate recovery of cells for rejuvenation or replacement.

Tests revealed that cells from enrichment cultures could be immobilized using sodium alginate $(3 \% \mathrm{w} / \mathrm{v})$ and would produce ethanol from carbon monoxide (see Figure 13). Alginic acid and carrageenan (Type I and Type II) were tried as immobilizing agents but were inferior to sodium alginate. Thus, immobilized cell technology appears to be a viable alternative to the conventional suspended cell mode of fermentation.

\section{Cultures That Produce Ethanol from Carbon Monoxide at $90^{\circ} \mathrm{C}$}

The possibility of utilizing a hyperthermophile to convert carbon monoxide in syngas to ethanol offers many attractive characteristics in making the conversion economically practicable.

Hyperthermophiles or extreme thermophiles are Archaea that have optimum growth temperatures above $80^{\circ} \mathrm{C}$. If hyperthermophiles that produce ethanol from 
carbon monixide could be found, they would offer a number of advantages in the production of ethanol from syngas. In the first place, these organisms should exhibit a faster rate of ethanol production. Furthermore, they would reduce the cost of cooling the gas (containing the carbon monoxide) going to the fermentor and would reduce the cost of recovering ethanol from the fermentor fluid if distillation is used as the recovery method since the boiling point of ethanol is $78.5^{\circ} \mathrm{C}$. Perhaps the most important characteristic of operating at a hyperthermophilic temperature is a major reduction in the possibility of contamination of the fermentation system since very few microorganisms can survive these temperatures, let alone grow.

The opportunity arose to try to determine if hyperthermophiles that can convert carbon monoxide to ethanol exist when cuttings from an oil well drilled to a depth of $11,000 \mathrm{ft}$ (where the temperature is $90^{\circ} \mathrm{C}$ ) became available. Ten mls of production water and cuttings (inoculum) were added to $140 \mathrm{ml}$ of mineral salts medium containing no carbon source and nitrate as a nitrogen source. Several $500 \mathrm{ml}$ boiling flasks with thermowells were heated on Variac-controlled heating mantles (Figure 14). A thermometer was inserted into the thermowell and the temperature monitored. Two of the enrichments were maintained at $90^{\circ} \mathrm{C}$ and one at $80^{\circ} \mathrm{C}$. Initially, pure carbon monoxide was added to the $350 \mathrm{ml}$ headspace above the enrichment. After several months of incubation, the headspace was replaced with syngas. Gram stains revealed the presence of cells in the $90^{\circ} \mathrm{C}$ enrichment culture (see Figure 15). Since the method of incubation described above was cumbersome and left a lot to be desired, it was decided to employ an oven as the incubator. Unfortunately, there is fluctuation in temperature in the oven causing the cultures to remain in the lag phase of growth rather than proceed to grow in the logarithmic phase of growth. This problem was solved by placing a quart fruit jar in a one-gallon tin can filled with sand and placing the culture vials in the jar or by burying the vials in a pan of sand as shown in Figure 16. Good growth of a carbon monoxide-utilizing enrichment culture at $90^{\circ} \mathrm{C}$ through seven subcultures has been achieved. Microscopic examination of the culture revealed only one morphological type of cell, but it is not certain that it is a pure culture on that basis alone. 
A series of enrichments was prepared using the cuttings from the oil well in $70 \mathrm{ml}$ glass vials fitted with butyl rubber stoppers and secured with aluminum caps as described previously. As shown in Figure 17, sixteen of the 21 enrichment cultures were able to produce ethanol from carbon monoxide. In fact, when the rate of ethanol production for these cultures was calculated over a period of $72 \mathrm{hrs}$, rather than during logarithmic growth, the rate was three times faster than the fastest rate reported in the literature.

Since conventional microbiological methodology (picking isolated colonies from the surface of agar plates) cannot be employed at $90^{\circ} \mathrm{C}$, other methods have to be employed, namely, dilution to extinction or using a solidifying agent capable of remaining solid at $90^{\circ} \mathrm{C}$.

Repeated attempts to isolate a pure culture using the dilution to extinction method were unsuccessful. Apparently, the slow growth of the ethanol-producing cultures allowed the faster-growing contaminants to outnumber the ethanol producers before carbon monoxide utilization was evident and dilutions made.

In previous studies on autotrophs, silica gel plates prepared with Ludox LS were sometimes employed in place of agar since organic matter inhibited some of the autotrophs. Ludox LS, a colloidal silica (Dupont), was employed to prepare silica gel plates according to the method described by Temple (1949) but cracked quickly due to the high temperature of incubation.

Another material employed to prepare a solid medium for use at high temperatures is Phytagel ${ }^{\mathrm{TM}}$ (Sigma). As with Ludox LS, the concentration of ions is critical. If the ionic strength is too high, cracks form in the medium, but if the ionic strength is too low the medium is too soft for streaking. Obviously, the plates must be kept in a closed system to prevent rapid water loss. A composition of nutrients was found that allowed the Phytage $\mathrm{T}^{\mathrm{TM}}$ to remain solid for nearly a month at $85^{\circ} \mathrm{C}$, but no growth occurred when streaked with enrichment cultures. This suggests that the nutrient composition was not suitable for colony formation. Even if the solid medium is nutritionally the same as the liquid medium, it has been shown that some microorganisms will not grow on a solid medium with the same nutrient content as the liquid medium (Brown, Strawinski, and McCleskey, 1964). 


\section{SUMMARY AND CONCLUSIONS}

The overall objective of this research effort was to determine if ethanol could be economically produced from carbon monoxide. While it was not the intent of this project to perform an economic evaluation, the project was intended to develop facts to help estimate the economics of the process. Evidence was obtained that suggested that there are microorganisms in the environment capable of producing ethanol at a rate greater than the best rate reported in the literature. Other studies clearly demonstrated that the cost of media could be reduced appreciably. However, a major problem was encountered in trying to maintain purity of the cultures. Also, various manipulations of the medium did not result in any major improvement in yield of ethanol for any of the cultures growing at ambient temperature.

Therefore, employing conventional technology as described in the literature for producing ethanol from carbon monoxide does not appear to be economically practicable. If, on the other hand, a different process, namely, immobilized cell technology, coupled with a more effective cell production process were employed, a more attractive economic outcome might be obtained. Toward this goal it was found that carbon monoxide-grown cells could be immobilized using $3 \%(\mathrm{w} / \mathrm{v})$ sodium alginate and the cells did produce ethanol. Obviously, harvesting cells from liquid media is a costly process and other means of growing cells could reduce the cost of the process. It was found that cell yield could be increased 100 to 1000 times using biphasic growth, thus making immobilized cell technology more attractive.

Another possibility for making the production of ethanol from carbon monoxide an economic possibility was the finding that a hyperthermophile was able to produce ethanol from carbon monoxide. Microorganisms capable of producing ethanol from carbon monoxide at $90^{\circ} \mathrm{C}$ were obtained from cuttings from an oil-drilling operation. These drill cuttings were obtained from a well being drilled at $11,000 \mathrm{ft}$ where the temperature was $90^{\circ} \mathrm{C}$. Some of the cultures from the cuttings produced ethanol more than three times faster than the fastest rate of ethanol production reported in the literature. Furthermore, the cost of reducing the temperature of syngas to the operational temperature for growing the ethanol-producing cultures to $90^{\circ} \mathrm{C}$, rather than approximately $30^{\circ} \mathrm{C}$, would be a cost savings. Also, if distillation were the method used 
to recover ethanol from the medium, additional savings would be realized since ethanol has a boiling point of $78.5^{\circ} \mathrm{C}$ and no additional heat would be needed for the ethanol recovery. An important consideration is the reduction in the possibility of contamination since very few microorganisms are able to survive at $90^{\circ} \mathrm{C}$, let alone grow.

On the basis of this research project it is concluded that there is a possibility of producing ethanol from the carbon monoxide in syngas using a hyperthermophilic, ethanol-producing microorganism rather than a mesophilic microbe. Further, using a biphasic method of growing cells coupled with immobilized cell technology would also help make the process more attractive economically. 


\section{REFERENCES}

1. Brown, L.R., R.J.Strawinski, and C.S. McCleskey. 1964. The isolation and characterization of Methanomonas methanooxidans. Can. J. Mic. 10:791.

2. Temple, K.L. 1949. A new method for the preparation of silica gel plates. J. Bacteriol., 57, 383. 


\section{FIGURES}

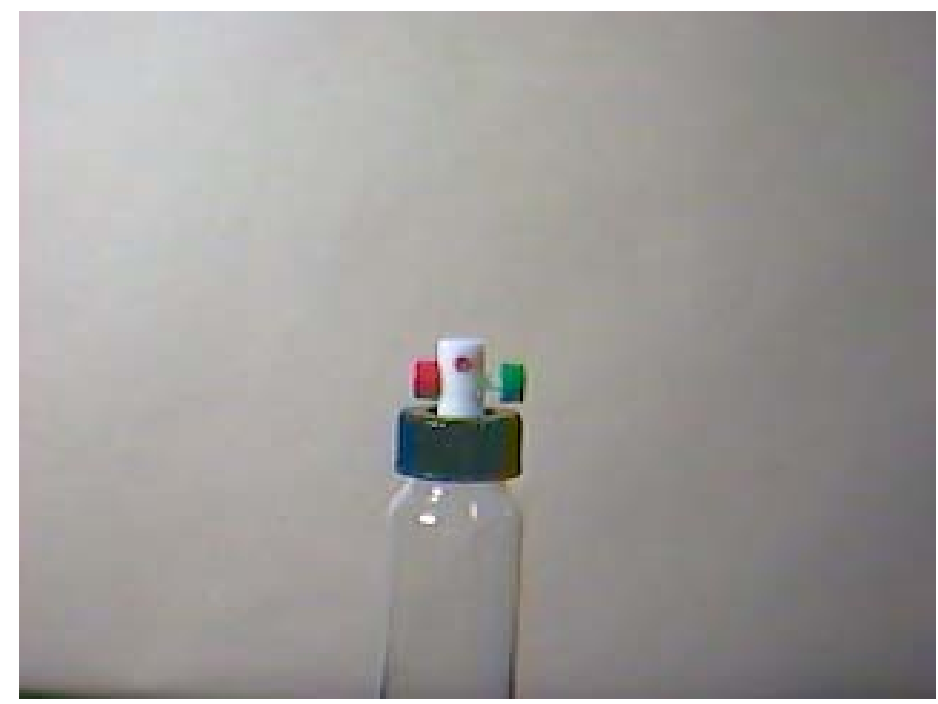

Figure 1. Sixty $\mathrm{ml}$ glass vial with miniert valve.

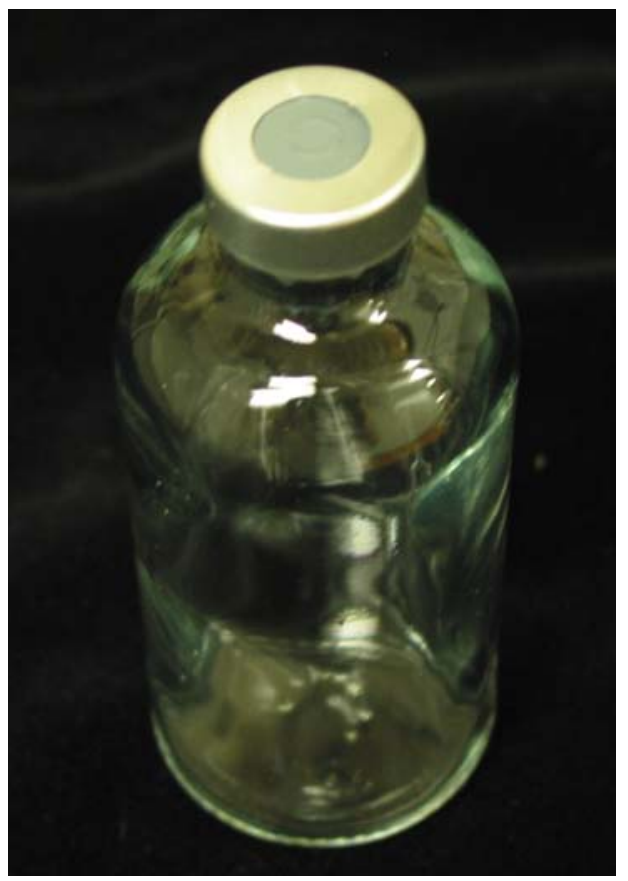

Figure 2. Seventy $\mathrm{ml}$ Wheaton vial with grey butyl stopper secured by an aluminum cap. 


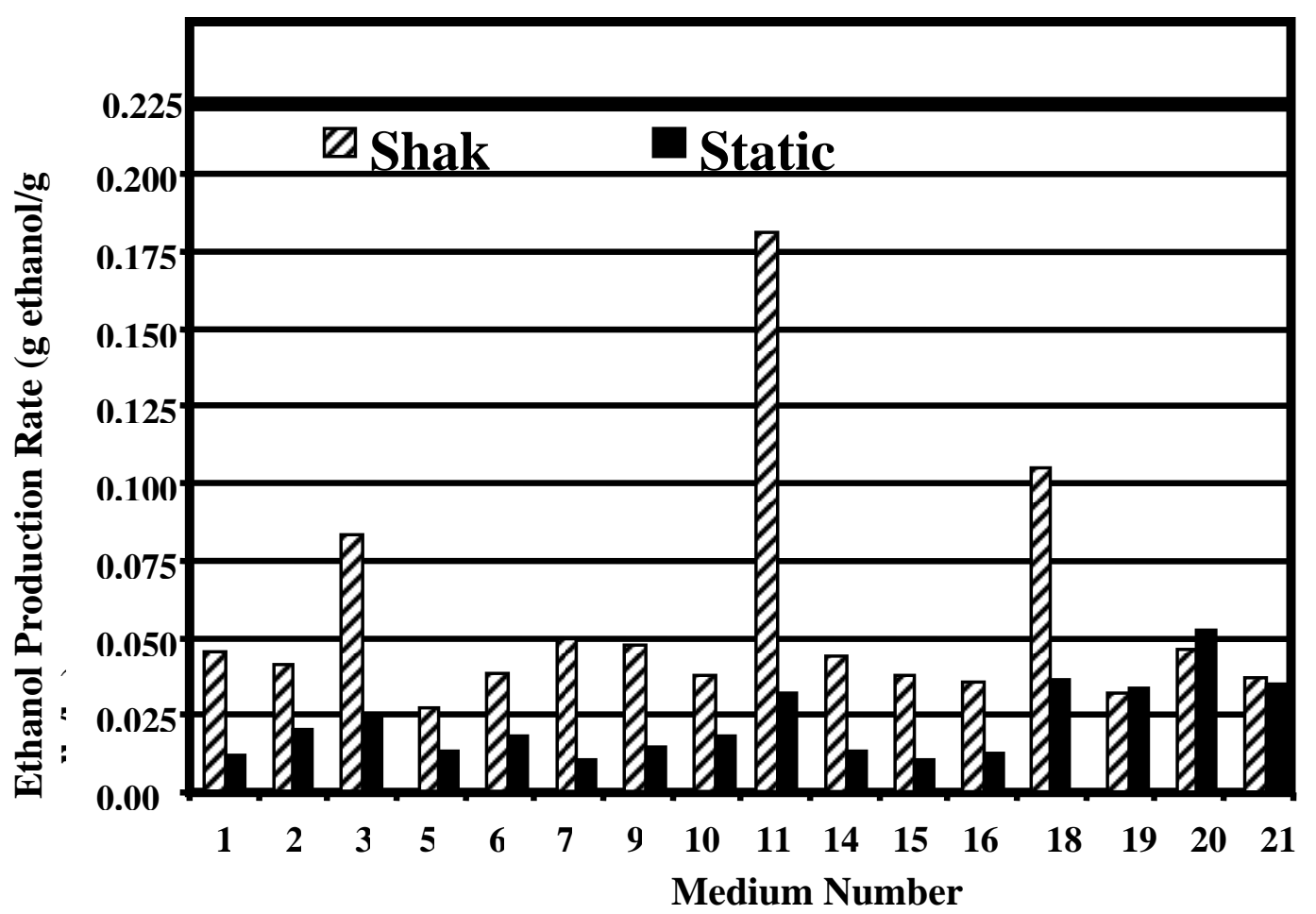

Figure 3. Rate of ethanol production for hog waste lagoon sludge after $\mathbf{7 2}$ hours of incubation.

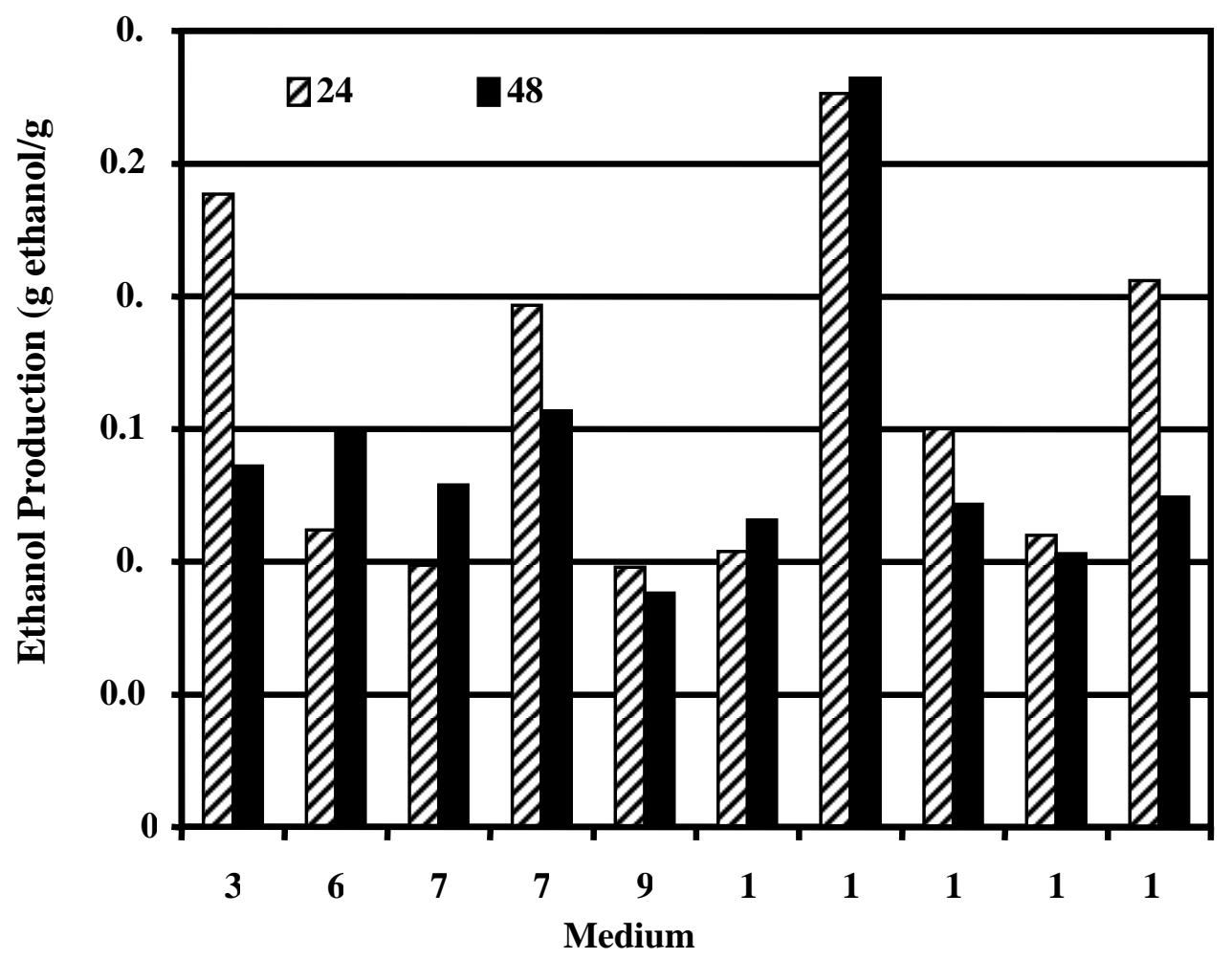

Figure 4. Ethanol production by enrichment cultures after 24 and 48 hours of incubation. 


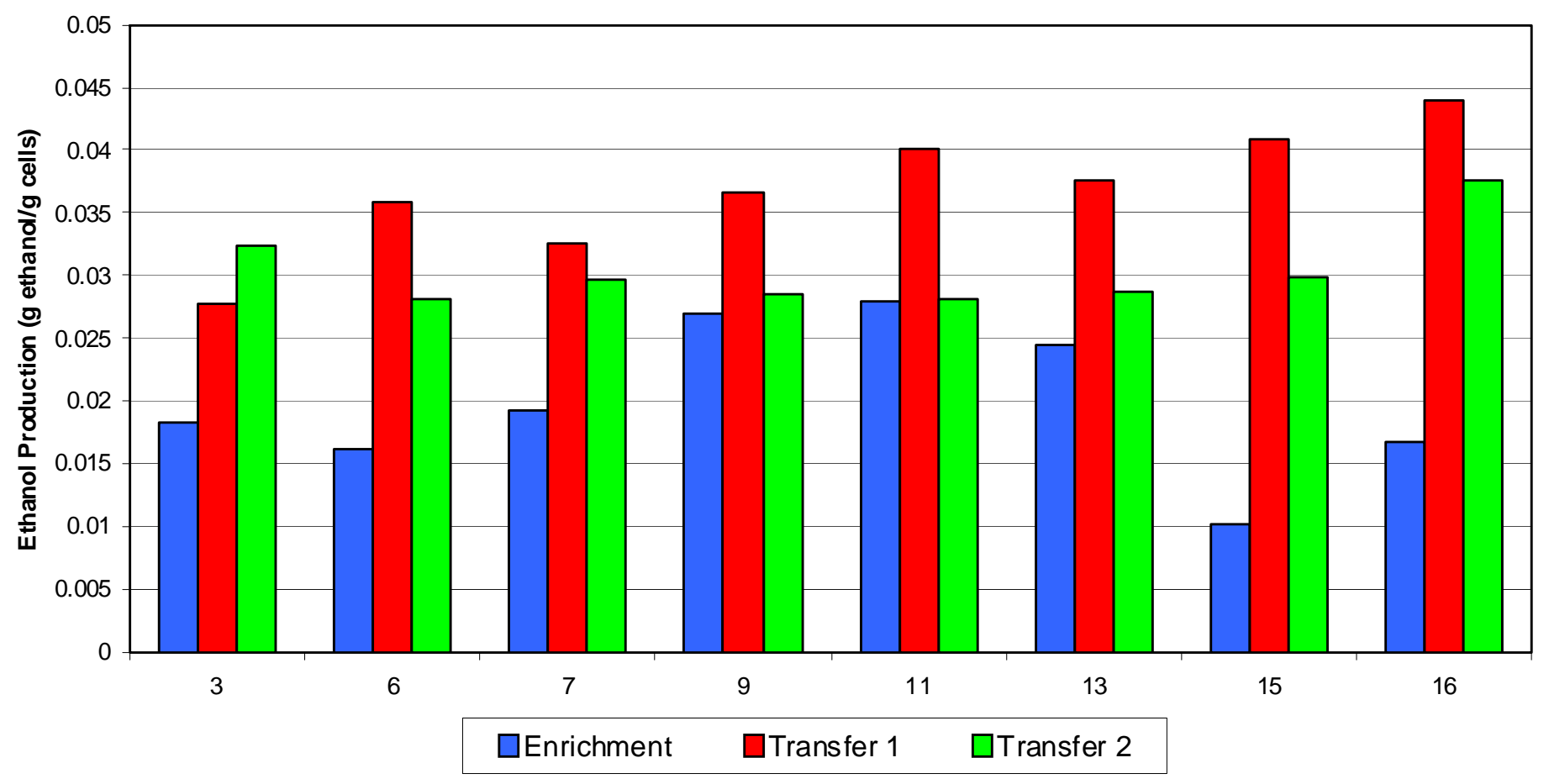

Figure 5. Ethanol production by enrichment cultures and transfers therefrom.

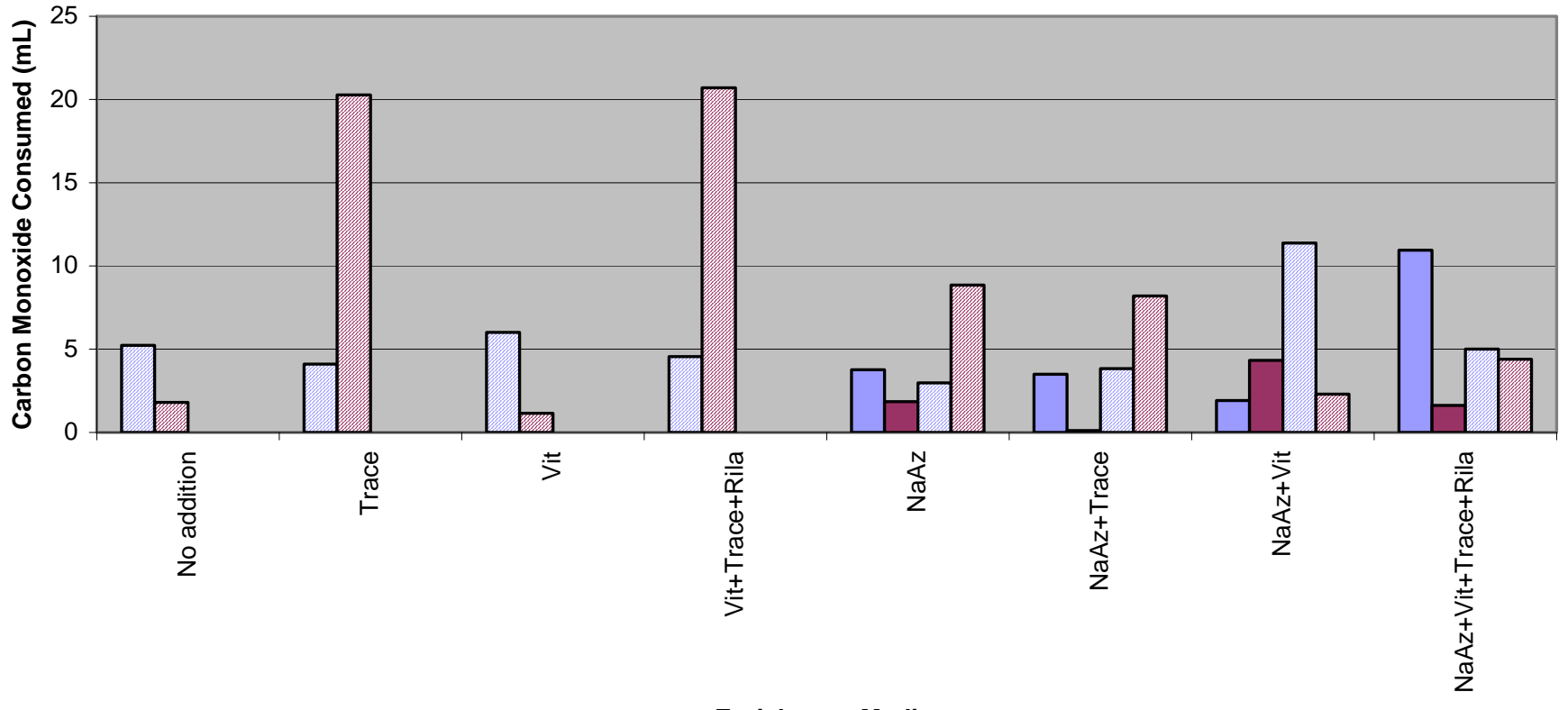

Enrichment Medium

$\square$ Static $\square$ Shake $\square$ Static (spores) $\square$ Shake (spores)

Figure 6. Carbon monoxide utilization by the culture in $10 \mathrm{ml}$ ethanol medium in an atmosphere containing carbon monoxide. 


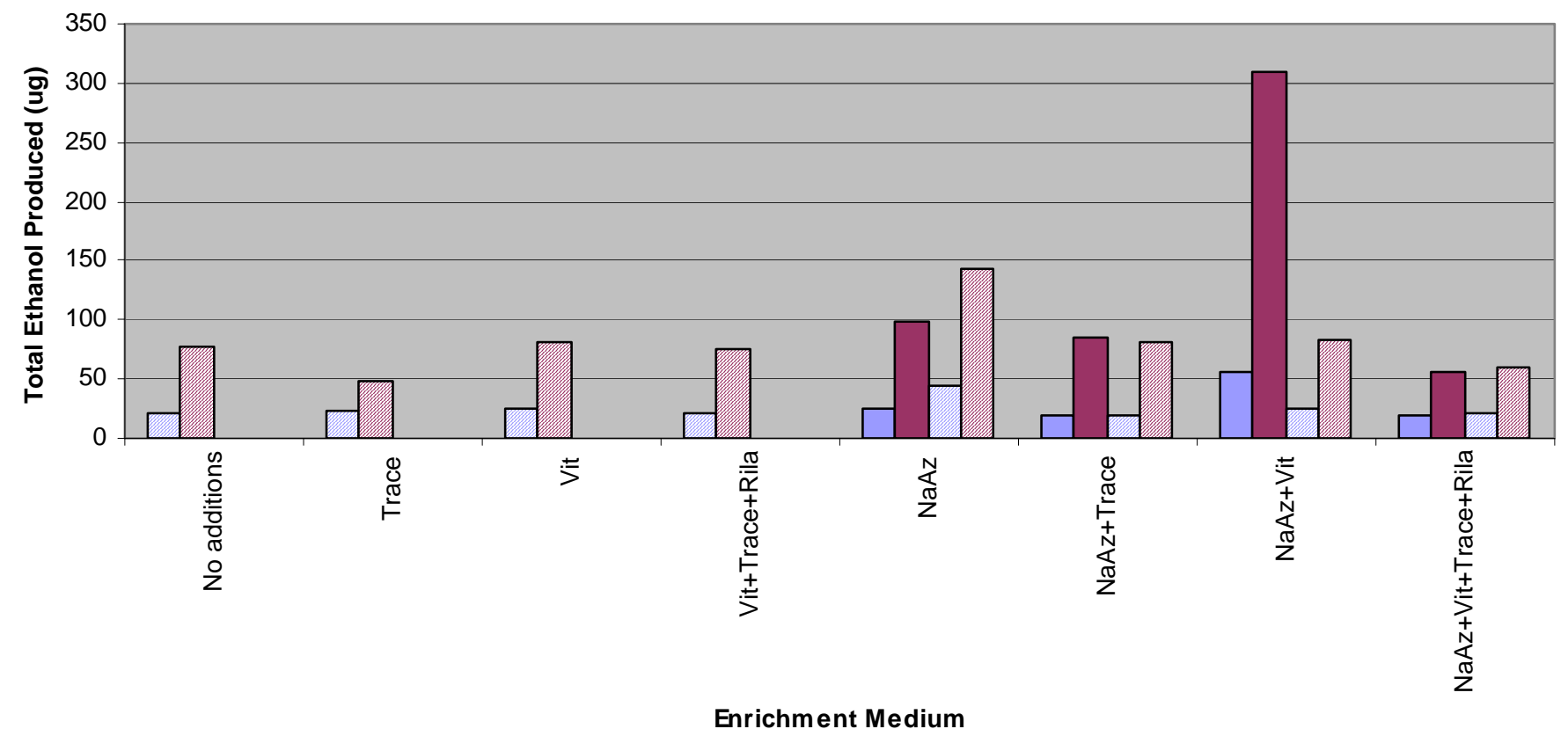

$\square$ static $\square$ shake $\square$ static (spores) $\square$ shake (spores)

Figure 7. Ethanol production by culture in $10 \mathrm{ml}$ ethanol medium in an atmosphere containing carbon monoxide.

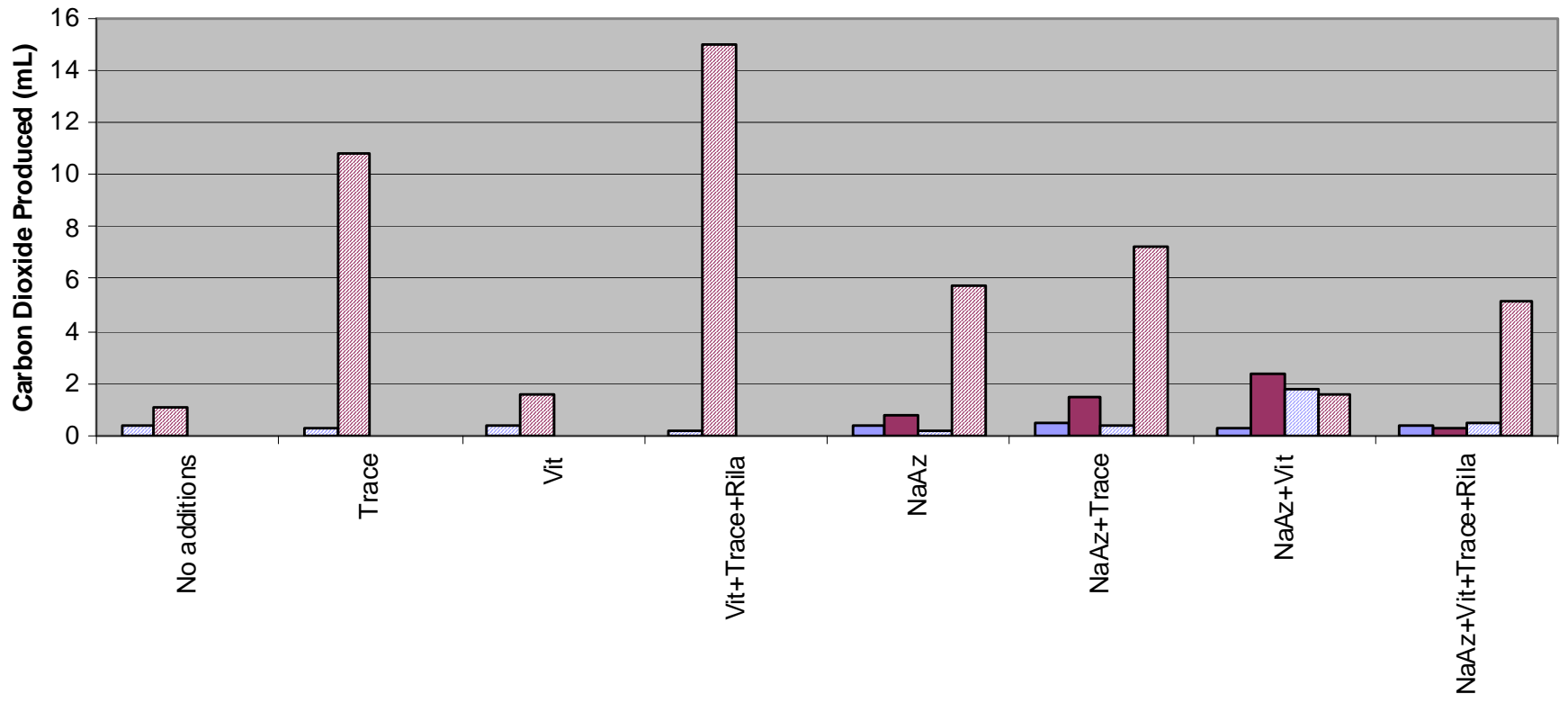

Enrichment Medium

$\square$ Static $\square$ Shake $\square$ Static (spores) $\square$ Shake (spores)

Figure 8. Carbon dioxide production by culture in $10 \mathrm{ml}$ ethanol medium in an atmosphere containing carbon monoxide. 


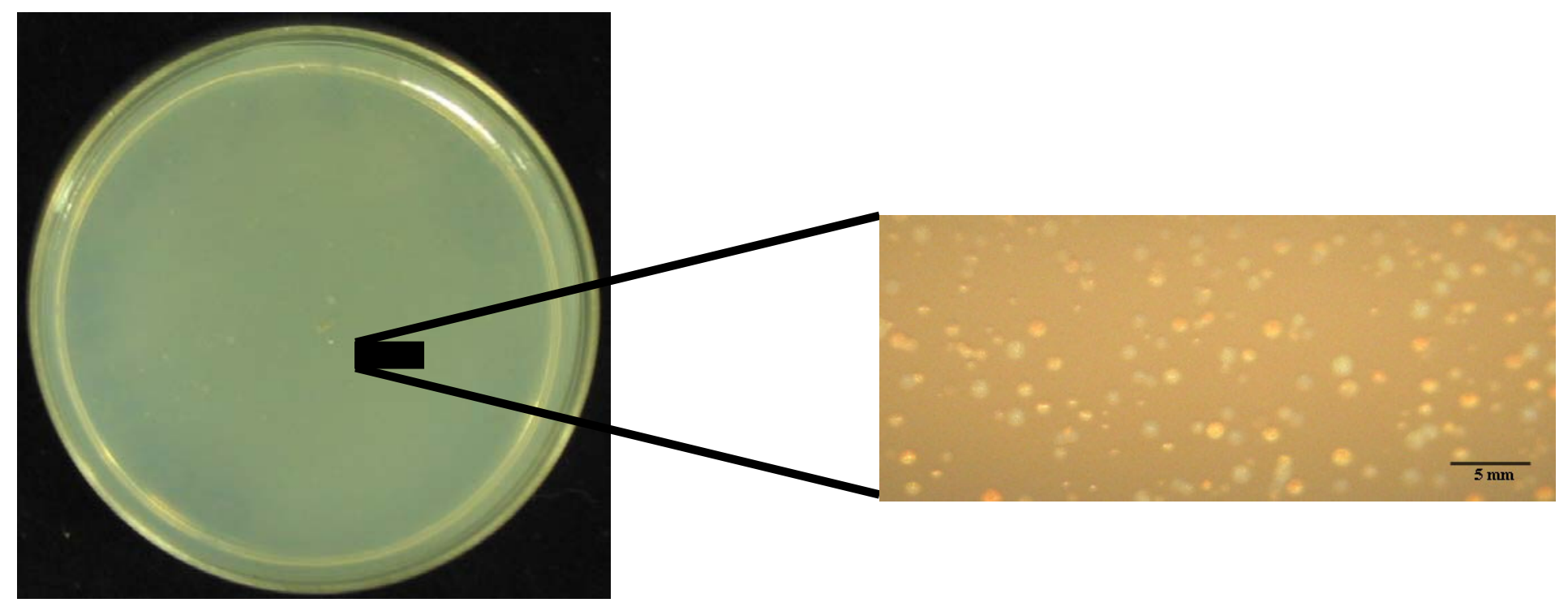

Figure 9. Colonies on MGM agar from enrichment cultures producing ethanol from Co.

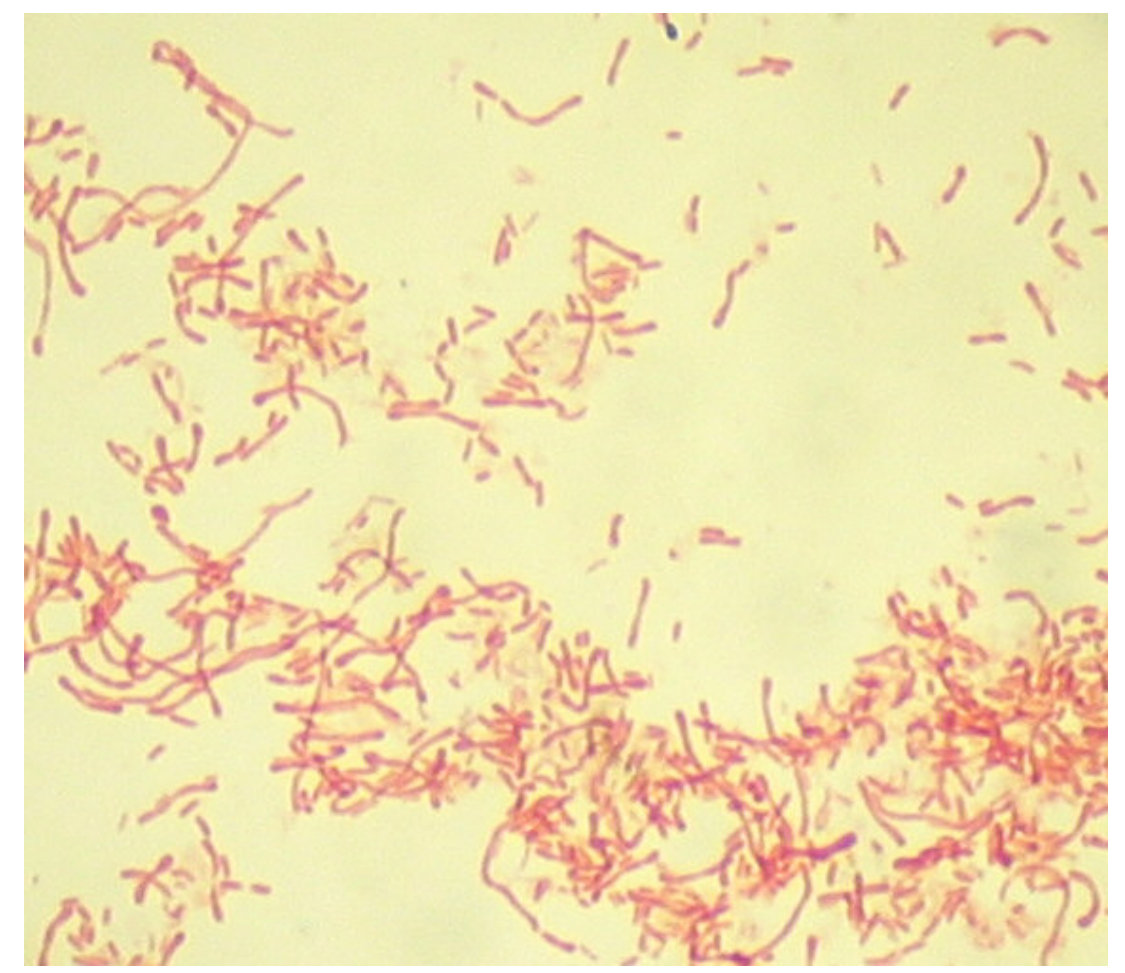

Figure 10. Culture A grown in syngas at $30^{\circ} \mathrm{C}$. 

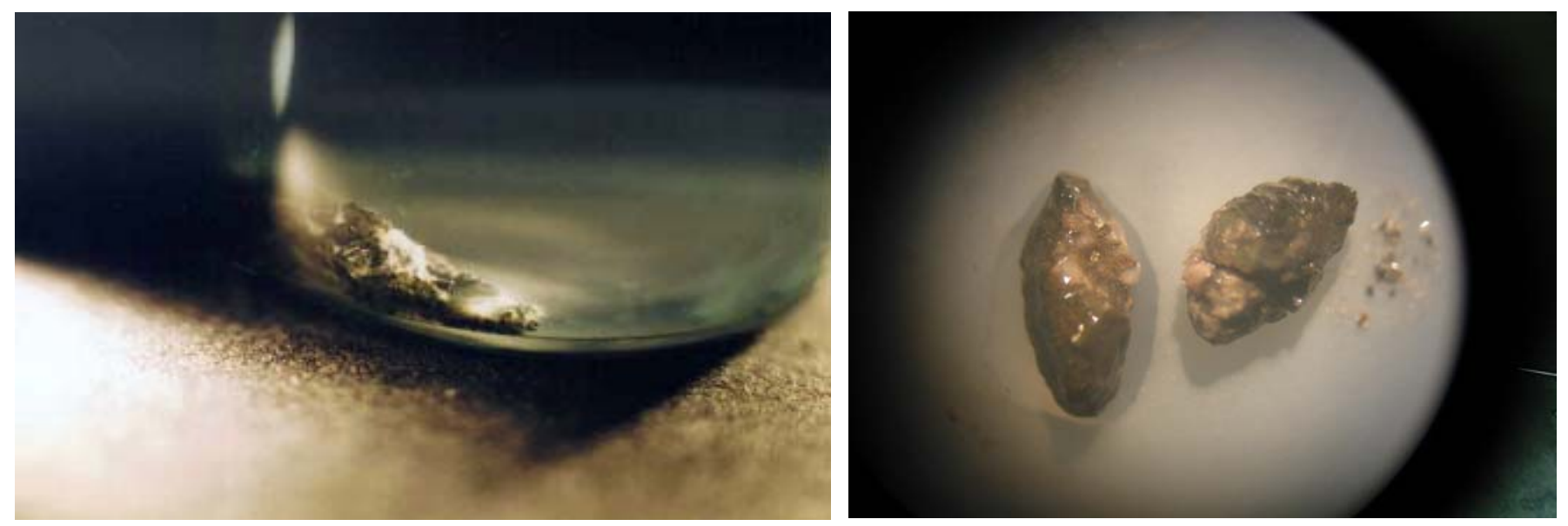

Figure 11. Growth on alabandite during incubation on syngas at $30^{\circ} \mathrm{C}$.

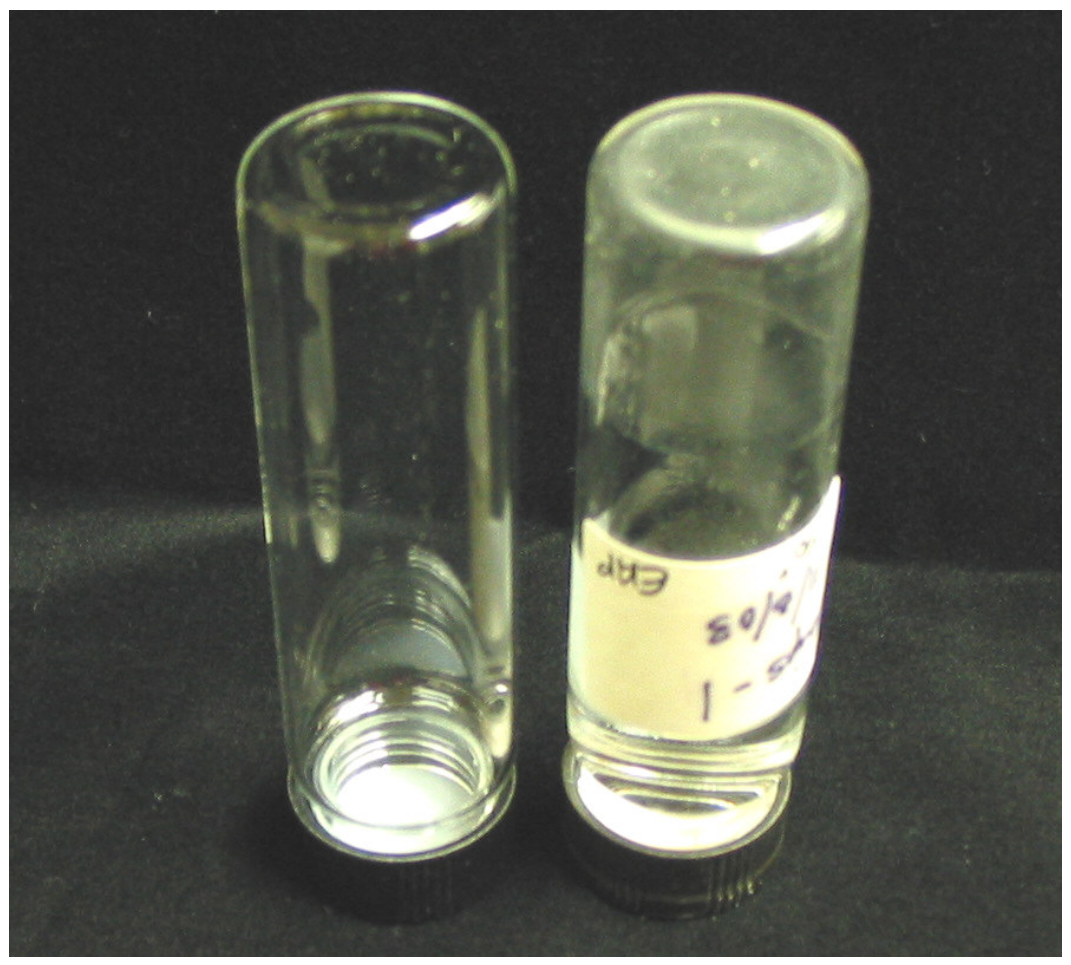

Figure 12. Growth attached to the glass test tube during incubation on syngas at $30^{\circ} \mathrm{C}$. 

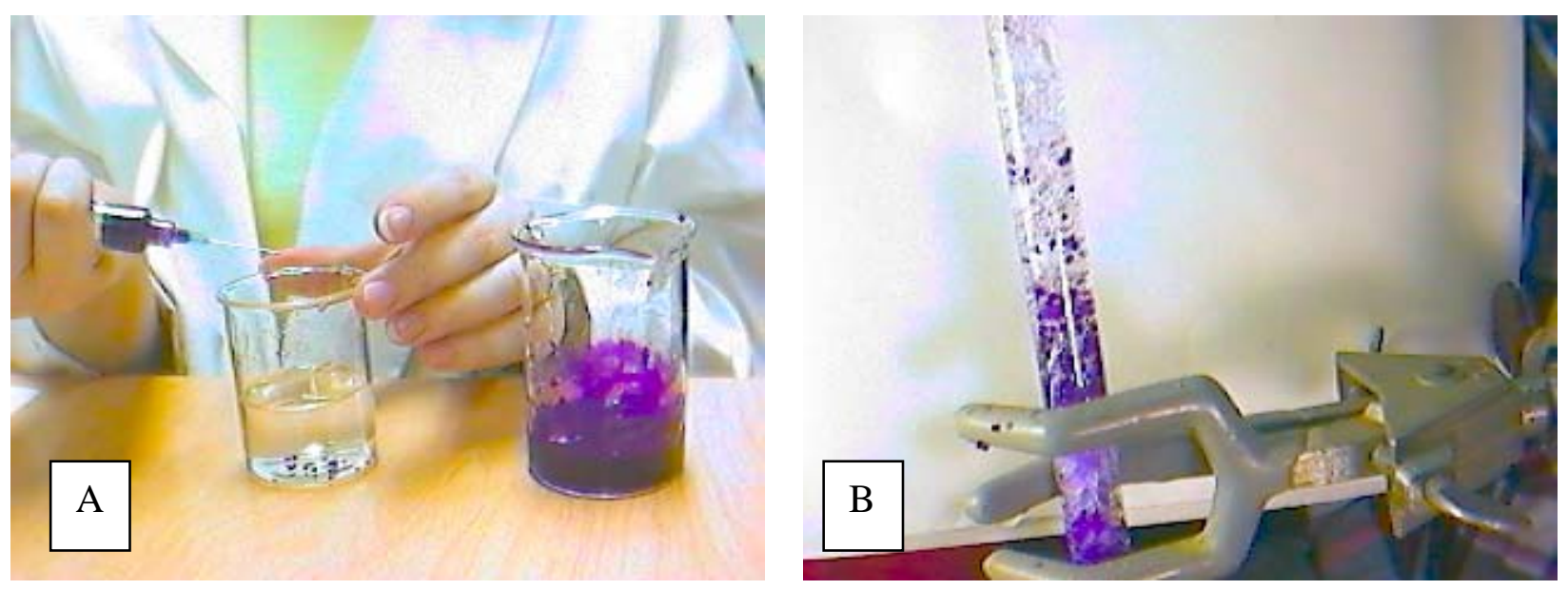

Figure 13. Cells producing ethanol from carbon monoxide entrapped in sodium alginate.

(A) Cells suspended in sodium alginate being dropped from a syringe into $0.1 \mathrm{M}$ $\mathrm{CaCl} 2$ solution to form small beads.

(B) Beads of sodium alginate containing ethanol producing cells in a glass tube with carbon monoxide being bubbled in from the bottom.

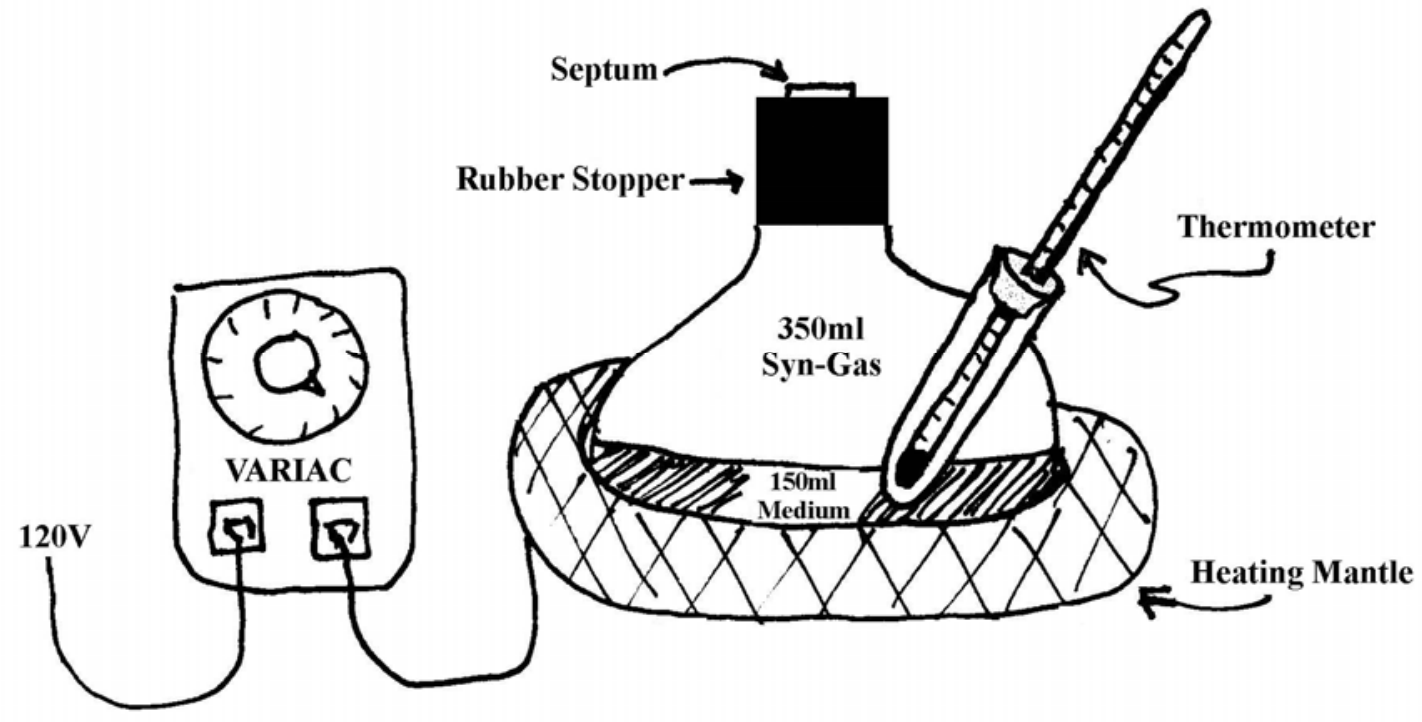

Figure 14. Enrichment culture setup. 


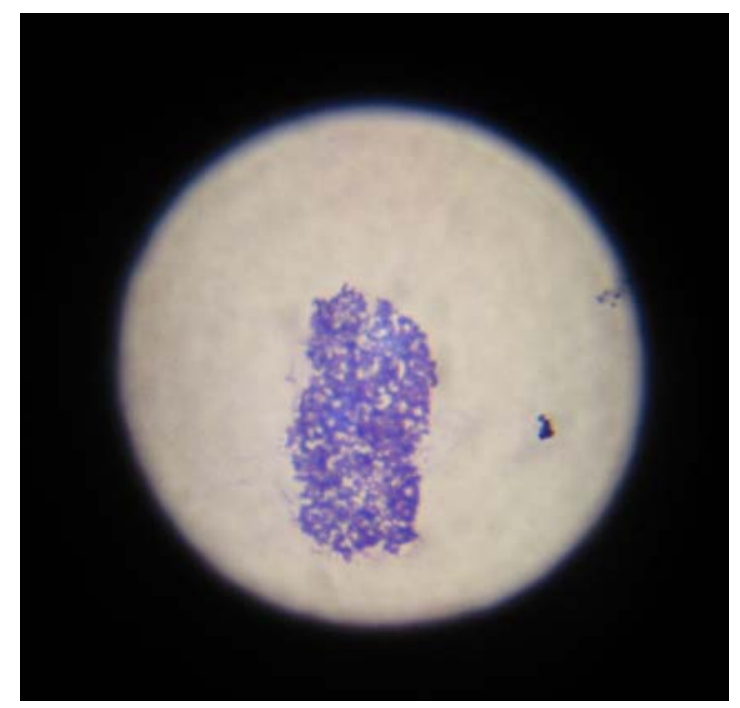

Figure 15. Photomicrograph of a $90^{\circ} \mathrm{C}$ culture taken from the sides of the culture vessel.
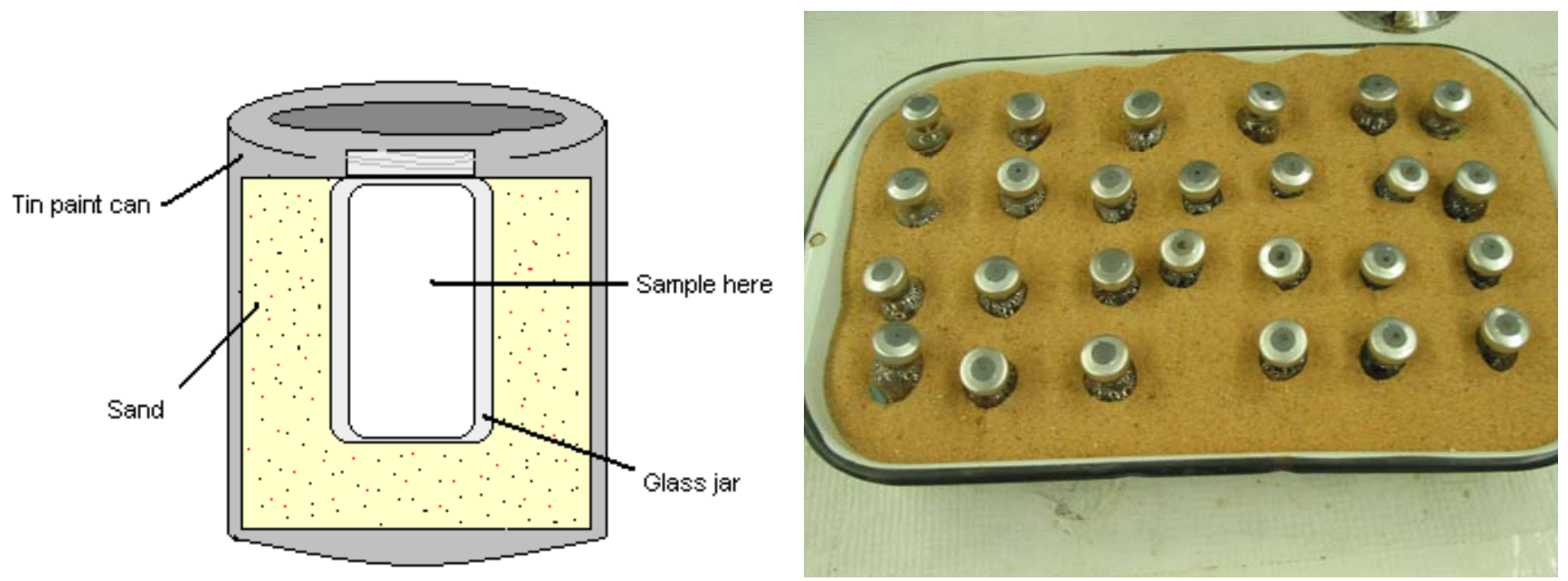

Figure 16. "Sand Cans" and "Sand Pans" help to maintain even temperature of cultures in oven. 


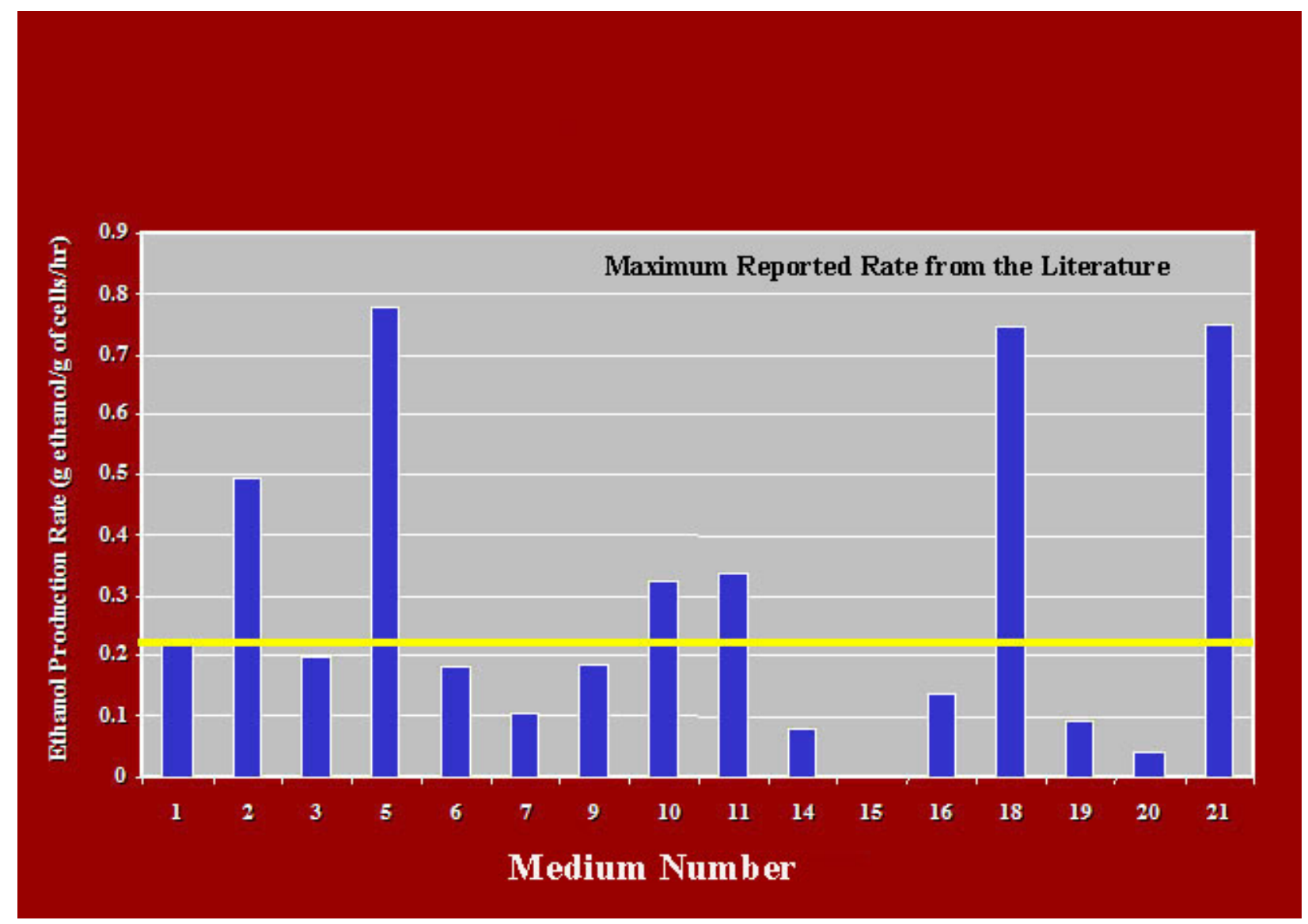

Figure 17. Rates of ethanol production by enrichment cultures from samples of drill cuttings from an oil well incubated at $90^{\circ} \mathrm{C}$. Rates calculated from a 72-hour period. 


\section{TABLES}

Table 1. Composition (per liter) of Modified ATCC Growth Medium 1754 (MGM)

\section{Basal Medium}

$\mathrm{NH}_{4} \mathrm{Cl}$

$1.0 \mathrm{~g}$

$\mathrm{KCl}$

$0.1 \mathrm{~g}$

$\mathrm{MgSO}_{4} \cdot 7 \mathrm{H}_{2} \mathrm{O}$

$0.2 \mathrm{~g}$

$\mathrm{NaCl}$

$0.8 \mathrm{~g}$

$\mathrm{KH}_{2} \mathrm{PO}_{4}$

$0.1 \mathrm{~g}$

$\mathrm{CaCl}_{2} \cdot 2 \mathrm{H}_{2} \mathrm{O}$

$20.0 \mathrm{mg}$

$\mathrm{NaHCO}_{3}$

Distilled water

$2.0 \mathrm{~g}$

Sterilized by autoclaving at $121^{\circ} \mathrm{C}$ for $15 \mathrm{~min}$.

$950.0 \mathrm{ml}$

\section{Trace Element Solution}

\begin{tabular}{|c|c|}
\hline Nitrilotriacetic acid & $2.0 \mathrm{~g}$ \\
\hline $\mathrm{MnSO}_{4} \cdot \mathrm{H}_{2} \mathrm{O}$ & $1.0 \mathrm{~g}$ \\
\hline $\mathrm{Fe}\left(\mathrm{SO}_{4}\right)\left(\mathrm{NH}_{4}\right)_{2} \cdot 6 \mathrm{H}_{2} \mathrm{O}$ & $0.8 \mathrm{~g}$ \\
\hline $\mathrm{CoCl}_{2} \cdot 6 \mathrm{H}_{2} \mathrm{O}$ & $0.2 \mathrm{~g}$ \\
\hline $\mathrm{ZnSO}_{4} \cdot 7 \mathrm{H}_{2} \mathrm{O}$ & $0.2 \mathrm{~g}$ \\
\hline $\mathrm{CuCl}_{2} \cdot 2 \mathrm{H}_{2} \mathrm{O}$ & $20.0 \mathrm{mg}$ \\
\hline $\mathrm{NiCl}_{2} \cdot 6 \mathrm{H}_{2} \mathrm{O}$ & $20.0 \mathrm{mg}$ \\
\hline $\mathrm{Na}_{2} \mathrm{MoO}_{4} \cdot 2 \mathrm{H}_{2} \mathrm{O}$ & $20.0 \mathrm{mg}$ \\
\hline $\mathrm{Na}_{2} \mathrm{SeO}_{4}$ & $20.0 \mathrm{mg}$ \\
\hline $\mathrm{Na}_{2} \mathrm{WO}_{4}$ & $20.0 \mathrm{mg}$ \\
\hline
\end{tabular}

Sterilized by filtration through a $0.45 \mu$ Millipore filter.

Wolfe's Vitamin Solution

Biotin

$2.0 \mathrm{mg}$

Folic Acid

$2.0 \mathrm{mg}$

Pyridoxal $\mathrm{HCl}$

$10.0 \mathrm{mg}$

Lipoic A (Thioctica)

$5.0 \mathrm{mg}$

Riboflavin

$5.0 \mathrm{mg}$

Thiamine $\mathrm{HCl}$

$5.0 \mathrm{mg}$

Ca-D-Pantothenate

$5.0 \mathrm{mg}$

Cyanocobalamin

$0.1 \mathrm{mg}$

P-Aminobenzoic Acid

$5.0 \mathrm{mg}$

Nicotinic Acid

$5.0 \mathrm{mg}$

Sterilized by filtration through a $0.45 \mu$ Millipore filter. 
Table 1. Composition (per liter) of Modified ATCC Growth Medium 1754 (MGM) - continued.

\section{Fructose Solution}

Fructose

$50.0 \mathrm{~g}$

Distilled water

$200.0 \mathrm{ml}$

Sterilized by filtration through a $0.45 \mu$ Millipore filter.

\section{Reducing Agent Solution}

L-Cysteine $\cdot \mathrm{HCl} \cdot \mathrm{H}_{2} \mathrm{O}$

$4.0 \mathrm{~g}$

Distilled water

$100.0 \mathrm{ml}$

Sterilized by filtration through a $0.45 \mu$ Millipore filter.

\section{Complete Medium}

Basal Medium

$940.0 \mathrm{ml}$

Trace Element Solution

$20.0 \mathrm{ml}$

Wolf's Vitamin Solution

$10.0 \mathrm{ml}$

Fructose Solution

$20.0 \mathrm{ml}$

Reducing Agent Solution

$10.0 \mathrm{ml}$

$\mathrm{pH}$ adjusted to 5.9

Note: All solutions prepared in an anaerobic hood, mixed, and dispensed into sterile culture vessels. Solid media prepared by adding $17 \mathrm{~g}$ of Bacto Agar per I of medium. 
Table 2. Modified C. Iungdahlii and C. acetobutylicum medium for Ethanol Production (EPM).

\section{Basal Mineral Medium}

$\left(\mathrm{NH}_{4}\right)_{2} \mathrm{SO}_{2}$

$1.0 \mathrm{~g}$

$\mathrm{NH}_{4} \mathrm{Cl}$

$1.0 \mathrm{~g}$

$\mathrm{KH}_{2} \mathrm{PO}_{4}$

$1.3 \mathrm{~g}$

Distilled water

$1000.0 \mathrm{ml}$

Sterilized by autoclaving at $121^{\circ} \mathrm{C}$ for $15 \mathrm{~min}$.

\section{Vitamin Solution}

Biotin

$20.0 \mathrm{mg}$

Folic Acid

$20.0 \mathrm{mg}$

Pyridoxal $\mathrm{HCl}$

$10.0 \mathrm{mg}$

Lipoic A (Thioctica)

$60.0 \mathrm{mg}$

Riboflavin

$50.0 \mathrm{mg}$

Thiamine $\mathrm{HCl}$

$50.0 \mathrm{mg}$

Ca-D-Pantothenate

$50.0 \mathrm{mg}$

Cyanocobalamin

$50.0 \mathrm{mg}$

P-Aminobenzoic Acid

$50.0 \mathrm{mg}$

Nicotinic Acid

$50.0 \mathrm{mg}$

Sterilized by filtration through a $0.45 \mu$ Millipore filter.

\section{Trace Mineral Solution}

Nitrolotriacetate

$1.5 \mathrm{~g}$

$\mathrm{MgSO}_{4} \cdot 7 \mathrm{H}_{2} \mathrm{O}$

$6.1 \mathrm{~g}$

$\mathrm{NaCl}$

$1.0 \mathrm{~g}$

$\mathrm{FeSO}_{4} \cdot 7 \mathrm{H}_{2} \mathrm{O}$

$0.1 \mathrm{~g}$

$\mathrm{CoCl}_{2} \cdot 6 \mathrm{H}_{2} \mathrm{O}$

$0.1 \mathrm{~g}$

$\mathrm{CaCl} \cdot 2 \mathrm{H}_{2} \mathrm{O}$

$0.1 \mathrm{~g}$

$\mathrm{ZnCl}_{2}$

$0.1 \mathrm{~g}$

$\mathrm{CuCl}_{2} \cdot 2 \mathrm{H}_{2} \mathrm{O}$

$0.01 \mathrm{~g}$

$\mathrm{AIK}\left(\mathrm{SO}_{4}\right)_{2} \cdot 12 \mathrm{H}_{2} \mathrm{O}$

$0.01 \mathrm{~g}$

$\mathrm{H}_{3} \mathrm{BO}_{3}$

$0.01 \mathrm{~g}$

$\mathrm{Na}_{2} \mathrm{MoO}_{4} \cdot 2 \mathrm{H}_{2} \mathrm{O}$

$0.01 \mathrm{~g}$

$\mathrm{NiCl}_{2} \cdot 6 \mathrm{H}_{2} \mathrm{O}$

$0.01 \mathrm{~g}$

$\mathrm{Na}_{2} \mathrm{SeO}_{3}$

$0.0005 \mathrm{~g}$

$\mathrm{MnSO}_{4} \cdot \mathrm{H}_{2} \mathrm{O}$

$0.5 \mathrm{~g}$

Distilled water

$2000.0 \mathrm{ml}$

Sterilized by filtration through a $0.45 \mu$ Millipore filter. 
Table 2. Modified C. Iungdahlii and C. acetobutylicum medium for Ethanol Production (EPM) - continued.

\section{Complete Medium}

Basal Mineral Medium

$970.0 \mathrm{ml}$

Vitamin Solution

$20.0 \mathrm{ml}$

Trace Mineral Solution

$10.0 \mathrm{ml}$

$\mathrm{pH}$ adjusted to 4.5

Note: All solutions prepared in an anaerobic hood, mixed, and dispensed into sterile culture vessels.

Table 3. Composition of Marine Broth.

\begin{tabular}{lc} 
Compound & Amount \\
\hline $\mathrm{NaCl}$ & $19.4500 \mathrm{~g} / \mathrm{l}$ \\
$\mathrm{MgCl}_{2}$ & $5.9000 \mathrm{~g} / \mathrm{l}$ \\
$\mathrm{Na}_{2} \mathrm{SO}_{4}$ & $3.2400 \mathrm{~g} / \mathrm{l}$ \\
$\mathrm{CaCl}_{2}$ & $1.8000 \mathrm{~g} / \mathrm{l}$ \\
$\mathrm{KCl}$ & $0.5500 \mathrm{~g} / \mathrm{l}$ \\
$\mathrm{NaHCO}_{3}$ & $0.1600 \mathrm{~g} / \mathrm{l}$ \\
$\mathrm{SrCl}_{2} \cdot 6 \mathrm{H}_{2} \mathrm{O}$ & $0.0340 \mathrm{~g} / \mathrm{l}$ \\
$\mathrm{H}_{3} \mathrm{BO}_{3}$ & $0.0220 \mathrm{~g} / \mathrm{l}$ \\
$\mathrm{Sodium} \mathrm{Silicate}$ & $0.220 \mathrm{~g} / \mathrm{l}$ \\
$\mathrm{NaF}$ & $0.0040 \mathrm{~g} / \mathrm{l}$ \\
$\mathrm{NH}_{4} \mathrm{NO}_{3}$ & $0.0024 \mathrm{~g} / \mathrm{l}$ \\
$\mathrm{Na}_{2} \mathrm{HPO}_{4}$ & $0.0016 \mathrm{~g} / \mathrm{l}$ \\
$\mathrm{Distilled}$ water & $0.0080 \mathrm{~g} / \mathrm{l}$
\end{tabular}


Table 4. Composition of Media Employed to Isolate Carbon Monoxide-Utilizing Microorganisms.

\begin{tabular}{|c|c|c|c|c|c|c|c|}
\hline $\begin{array}{l}\text { Medium } \\
\text { Number }\end{array}$ & $\begin{array}{l}\text { Basal } \\
\text { Mineral } \\
\text { Solution }^{1}\end{array}$ & Vitamins $^{2}$ & $\begin{array}{l}\text { Trace } \\
\text { Elements }\end{array}$ & $\begin{array}{l}\text { Brilliant } \\
\text { Green }^{4}\end{array}$ & Azide $^{5}$ & Oxgall $^{6}$ & Rila $^{7}$ \\
\hline 1 & BMS & + & + & - & - & - & - \\
\hline 2 & BMS & + & + & + & - & - & - \\
\hline 3 & BMS & + & + & - & + & - & - \\
\hline 4 & BMS & + & + & - & - & + & - \\
\hline 5 & BMS & + & - & - & - & - & - \\
\hline 6 & BMS & + & - & + & - & - & - \\
\hline 7 & BMS & + & - & - & + & - & - \\
\hline 8 & BMS & + & - & - & - & + & - \\
\hline 9 & BMS & - & + & - & - & - & - \\
\hline 10 & BMS & - & + & + & - & - & - \\
\hline 11 & BMS & - & + & - & + & - & - \\
\hline 12 & BMS & - & + & - & - & + & - \\
\hline 13 & BMS & - & - & - & - & - & - \\
\hline 14 & BMS & - & - & - & - & - & + \\
\hline 15 & BMS & - & - & + & - & - & + \\
\hline 16 & BMS & - & - & - & + & - & + \\
\hline 17 & BMS & - & - & - & - & + & + \\
\hline 18 & Marine 8 & - & - & - & - & - & - \\
\hline 19 & Marine & - & - & + & - & - & - \\
\hline 20 & Marine & - & - & - & + & - & - \\
\hline 21 & Marine & - & - & - & - & + & - \\
\hline
\end{tabular}

${ }^{1}$ Composed of $50 \mathrm{mg}\left(\mathrm{NH}_{4}\right)_{2} \mathrm{SO}_{4}, 50 \mathrm{mg} \mathrm{NH}{ }_{4} \mathrm{Cl}$, and $68 \mathrm{mg} \mathrm{KH}_{2} \mathrm{PO}_{4}$ per $100 \mathrm{ml}$ distilled water

${ }^{2} 2 \mathrm{ml}$ of the following solution contained in $100 \mathrm{ml}$ of medium: $20 \mu \mathrm{g}$ biotin, $20 \mu \mathrm{g}$ folic acid, $100 \mu \mathrm{g}$ pyridoxal $\mathrm{HCl}, 60 \mu \mathrm{g}$ lipoic acid, $50 \mu \mathrm{g}$ thiamine $\mathrm{HCl}, 50 \mu \mathrm{g}$ calcium pantothenate, $50 \mu \mathrm{g}$ cyanocobalamin, $50 \mu \mathrm{g} \mathrm{p}$-aminobenzoic acid, and $50 \mu \mathrm{g}$ nicotinic acid.

${ }^{3} 0.5 \mathrm{ml}$ of the following solution contained in $100 \mathrm{ml}$ of medium: $1.5 \mathrm{mg}$ nitrilotriacetate, $6.1 \mathrm{mg}$ $\mathrm{MgSO}_{4} \cdot 7 \mathrm{H}_{2} \mathrm{O}, 1 \mathrm{mg} \mathrm{NaCl}, 0.1 \mathrm{mg} \mathrm{FeSO}{ }_{4} \cdot 7 \mathrm{H}_{2} \mathrm{O}, 0.1 \mathrm{mg} \mathrm{CoCl} \cdot 2 \mathrm{H}_{2} \mathrm{O}, 0.1 \mathrm{mg} \mathrm{CaCl} \cdot 2 \mathrm{H}_{2} 0,0.1 \mathrm{mg} \mathrm{ZnCl}$, $10 \mu \mathrm{g} \mathrm{CuCl}_{2} \cdot 2 \mathrm{H}_{2} \mathrm{O}, 50 \mu \mathrm{g} \mathrm{NiCl}_{2} \cdot 6 \mathrm{H}_{2} \mathrm{O}, 0.5 \mu \mathrm{g} \mathrm{Na}_{2} \mathrm{SeO}_{3}$, and $0.5 \mathrm{mg} \mathrm{MnSO}_{4} \mathrm{H}_{2} \mathrm{O}$.

${ }^{4}$ Medium contained $250 \mu \mathrm{g}$ brilliant green $/ 100 \mathrm{ml}$.

${ }^{5}$ Medium contained $5 \mathrm{mg}$ sodium azide/100 $\mathrm{ml}$.

${ }^{6}$ Medium contained $50 \mathrm{mg}$ Bacto oxgal/100 ml.

${ }^{7}$ Medium contained $133 \mathrm{mg}$ Rila Sea Salts $/ 100 \mathrm{ml}$.

${ }^{8}$ See Table 3. 
Table 5. Ethanol by Three Pure Culture Isolates.

\begin{tabular}{|c|c|}
\hline Culture Designation & g Etoh/g cells/hr \\
\hline C & 0.15 \\
\hline 4 & 0.94 \\
\hline A & 0.98 \\
\hline
\end{tabular}




\section{DEVELOPMENT OF SULFONATED ETHYLENE-VINYL ALCOHOL COPOLYMERS FOR MEMBRANE APPLICATIONS \\ Performer: University of Southern Mississippi}

\section{SUMMARY}

This work has focused on the synthesis and characterization of unique ionomer materials for application in membrane-based separations. Based on previous work in the Moore group, the ability to alter, and control, morphology will be used as a technique to develop new materials to achieve separation of acid-sugar and ethanolwater mixtures.

The most promising materials are sulfonated ethylene-vinyl alcohol copolymers $(E V O H)$. EVOH possesses a unique balance of hydrophilic, vinyl alcohol, and hydrophobic, ethylene, character making it interesting in barrier and separations applications. The incorporation of an ionic functionality into the already hydrophilic vinyl alcohol causes preferential swelling by water. Ionic functionality will also be advantageous for application in acid-sugar separations, as the fixed ionic species within the material will make it permselective to ionic species of opposite charge. In this application, the sulfonated EVOH is permselective to protons in an acid-sugar separation. Coupling this cation-selective material with an anion-selective membrane in an electrodialysis application makes possible ionic-nonionic separation, in this case acid-sugar separation.

Sulfonated $\mathrm{EVOH}$ has been produced through chemistry adopted from publications on poly (vinyl alcohol) materials. Using propane sultone under appropriate reaction conditions, the sulfonate functionality is introduced onto the polymer using a short alkyl tether (Figure 1) [1].

Observation of this modified material shows a drastic alteration to the hydrophilichydrophobic balance of the polymer. The water insoluble EVOH becomes water soluble upon sulfonation. However, one must note, if this polymer is to be used in separations of water containing species, it cannot be water soluble. As a result, the procedure has been altered to cross-link EVOH using a dialdehyde [2] prior to sulfonation. The resulting membrane retains the unique swelling characteristics yet is no longer water soluble, making it useful for membrane separations in aqueous media. 
As seen in Figure 2, dialdehyde cross-linking can be difficult to quantify at low cross-link densities; however, ATR FT-IR yields indirect evidence of cross-linking. The disappearance of the vinyl alcohol crystalline band at $1140 \mathrm{~cm}^{-1}$ and the relative decrease in the hydroxyl stretch at $-3200 \mathrm{~cm}^{-1}$ confirms cross-linking, the two bands indicating a decrease in crystallinity and quantity of hydroxyl groups in the sample, respectively.

Small angle X-ray scattering analysis of the sulfonated EVOH, conducted at the National Synchrotron Light Source at Brookhaven National Labs, shows that in the as processed state, sulfonate groups are more homogenously dispersed throughout the membrane, showing little evidence of the characteristic ionic aggregation seen in other sulfonated ionomers. The integrated intensity of the 2-D scattering profile shows no readily distinguishable ionomer peak at 2-4 nm-1, indicating little or no ionic aggregation (Figure 3). However, further work has shown that subsequent annealing of the polymer at elevated temperatures causes the sulfonate functionalities to aggregate into distinct ionic domains.

${ }^{23} \mathrm{Na}$-NMR has been used to examine the effect of annealing at elevated temperature on the state if ionic aggregation. As seen in Figure 4 the resonance at $\sim 7$ ppm attributed to lone ion pairs in the matrix disappears with annealing. Thus, it can be seen that annealing at $120^{\circ} \mathrm{C}$ provides sufficient mobility for ionic groups to cluster within the matrix leaving only the broad aggregate resonance centered around $\sim-12$ ppm. The ability to thermally induce ionic aggregation provides an alternative method to alter morphology in the existing membrane.

In addition to traditional dense membranes, the formation of asymmetric membranes has been accomplished using published techniques. Non-solvent Induced Phase Inversion, or NIPI, is used to produce asymmetric morphologies through the liquid-liquid demixing of a polymer solution immersed into a non-solvent bath. Asymmetric polysulfone membranes of varying morphology have been produced in this project through changes in the solvent medium, as shown in Figure 5.

An asymmetric polysulfone membrane cross-section is examined in the set of electron micrographs. The left micrograph shows the porous interior responsible for maintaining high flux through separation membranes. On the right, the dense 
membrane skin is responsible for selectivity. As a result, the asymmetric structure can maintain high selectivity yet achieve relatively high flux through the thin surface layer. The asymmetric structure thus provides a simple means of producing and supporting a delicate membrane of micron thickness.

The effect of solvent on membrane morphology has also been examined in this project. Here, membranes were prepared by dissolving in varying solvents, casting onto a glass substrate and immersing in a water bath to affect phase inversion. The resultant changes in morphology can be attributed to variations in the process of liquidliquid demixing thereby producing profound differences in morphology. Though not explored, literature has shown variations in morphology with non-solvents as well.

Figure 6 shows the differences in morphology that accompany changes in the solvent. Membranes cast from N,N-Dimethylformamide (DMF) show a highly porous interior composed of small spherical pores, whereas membranes cast from $\mathrm{N}$ methylpyrrolidinone (NMP) have a finger-like membrane interior. This result shows the flexibility in asymmetric membrane production that will be explored as a means to improve separations performance.

Early evaluations of membrane performance have been carried out using simple testing procedures while final equipment is under construction. Swelling experiments have been performed to examine the differential swelling of $\mathrm{EVOH}$ and sulfonated $\mathrm{EVOH}$ in water and ethanol. As shown in Figure 7, the incorporation of ionic functionalities has a drastic effect on the swelling behavior.

Sulfonated EVOH absorbs $\sim 12-14$ times its mass in water yet only absorbs $50 \%$ of its mass in ethanol, indicating preferential swelling. Considering sorption to be related to solubility and solubility to be a component of permeation this result provides promise that sulfonated EVOH will be permselective to water. Examination of vapor permeation through sulfonated EVOH supports this hypothesis, indicating water does in fact permeate at a faster rate than ethanol.

Examination of Figure 8 could lead to the assumption that the two components permeate the membrane at approximately the same rate. Though the apparent rate of mass transfer is similar for water and ethanol, considering the vapor pressure of each component, water permeates at nearly twice the rate of ethanol. In comparison, ethanol 
permeates through $\mathrm{EVOH}$ at a slightly higher rate, indicating sulfonation alters the permselectivity making the membrane applicable to ethanol dehydration. An increase in the permeation rate of each component accompanies the change in permselectivity following sulfonation. Whereas the water permselectivity can be attributed to the increased polar character introduced by the sulfonate groups, the overall increase in permeation rate is a synergistic effect of the ionic incorporation and the resultant drop in crystallinity.

The DSC traces of Figure 9 show the decrease in crystallinity with cross-linking and the near complete loss of crystalline material with sulfonation. In each case, chemical modification of the vinyl alcohol segments create defects that disrupt crystallizable segments. Given that crystalline regions are inaccessible to diffusing materials, the increased permeation rate for each species through sulfonated EVOH can also be linked to the decreased crystallinity that increases available volume for diffusion. One must note that the sample used in these permeation studies was not cross-linked, yet the conclusions above still apply.

Early evaluation of acid-sugar separation membranes has been accomplished through a diffusion measurement. Using a model cellulose hydrolyzate composed of $15 \mathrm{wt} \%$ sulfuric acid and $8 \mathrm{wt} \%$ glucose, the separation has been monitored by tracking the acid diffusing through the membrane and the glucose retained by the membrane.

As seen in the acid break out curves of Figure 10, acid permeates through sulfonated EVOH at approximately 5 times that of EVOH. In combination with the effects of ionic incorporation, the decreased crystallinity discussed above must be considered as influencing the rate of diffusion through the membrane. Analysis of the sugar retained during the measurement shows both the EVOH and sulfonated EVOH retain $\sim 80 \%$ of glucose showing each membrane to be effective in facilitating acid diffusion while retaining glucose. However, when the kinetics of the process is considered, it is noted that the sulfonated material allows more rapid acid diffusion, thereby making it more efficient at separation. At present, an electrodialysis system is being set up to achieve separation in a more controlled and more easily monitored method. 


\section{REFERENCES}

1. Goethals, E. J.; Natus, G. Die Makromolekulare Chemie 1968, 116, 152-157.

2. Sheilbey, D. W.; Manzo, M. A.; and Gonzalez-Sanabria, O. D. J. Electrochemical Society 1983, 130, 255-259. 


\section{FIGURES}

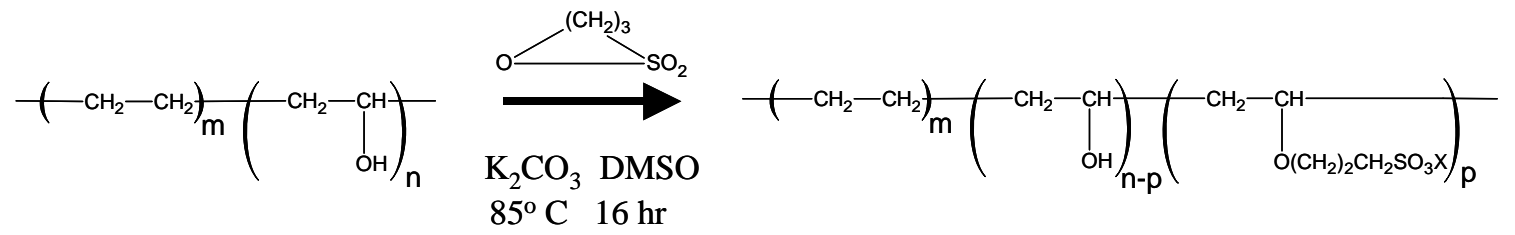

Figure 1. Sulfonation Reaction
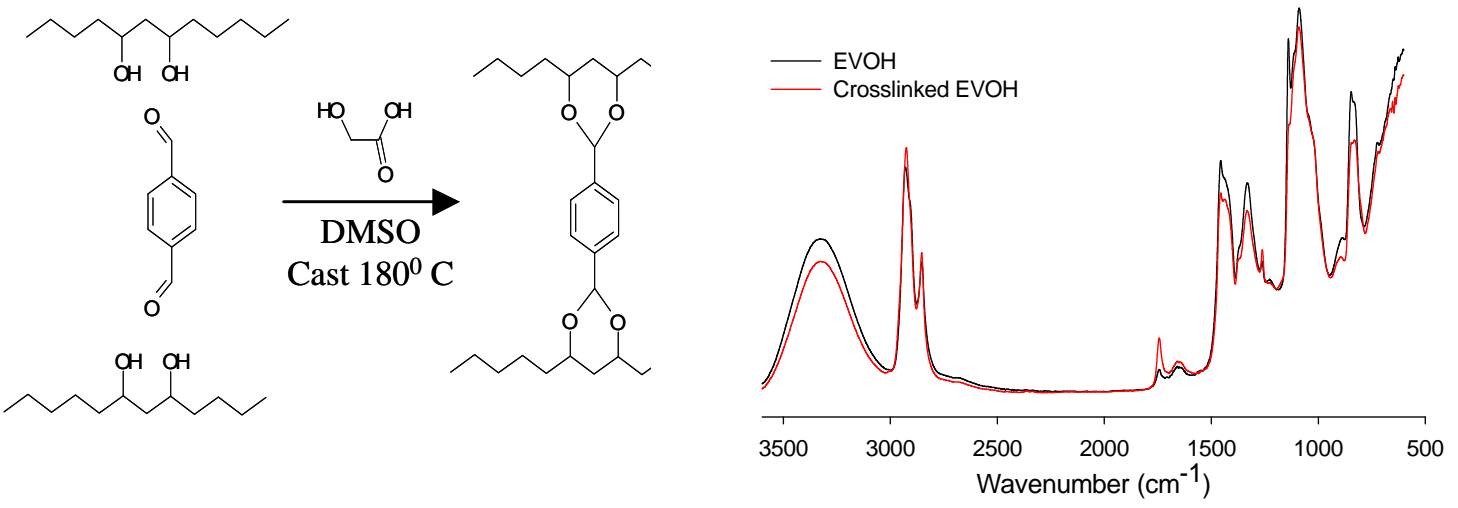

Figure 2. EVOH cross-linking using dialdehydes
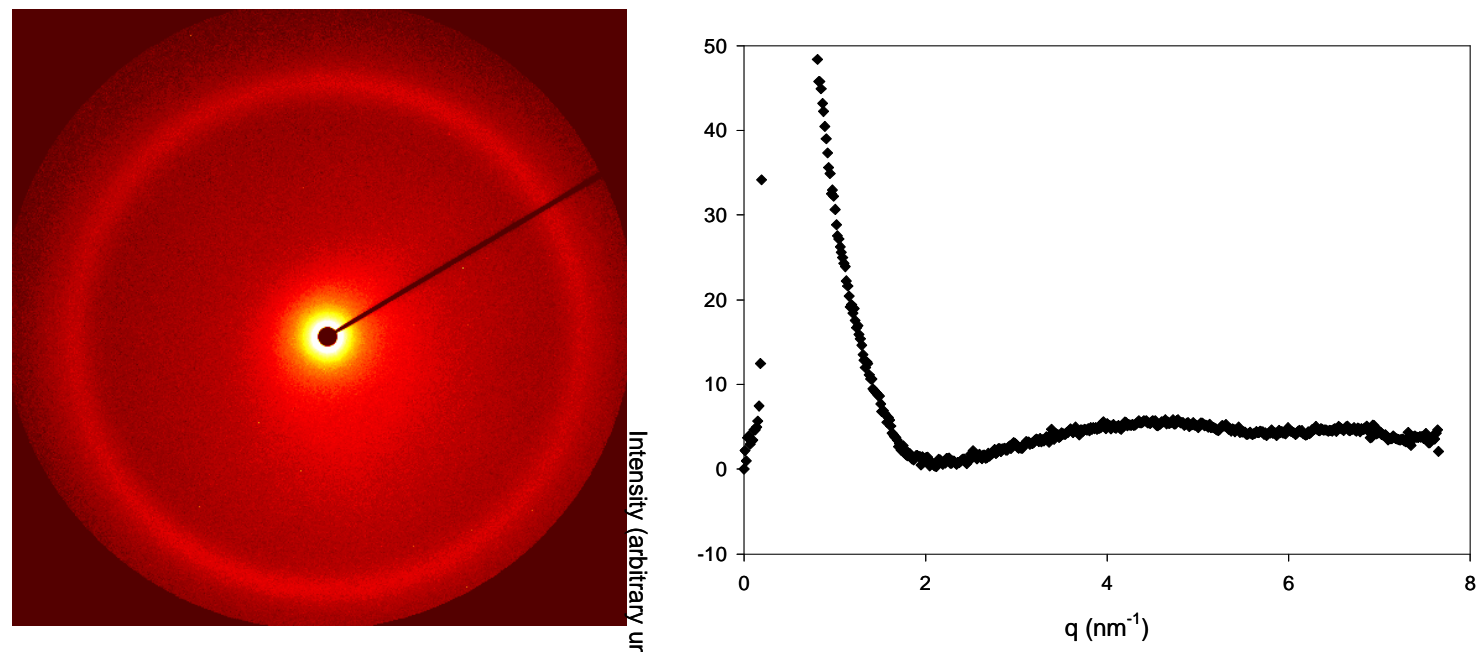

Figure 3. SAXS analysis of Sulfonated EVOH 


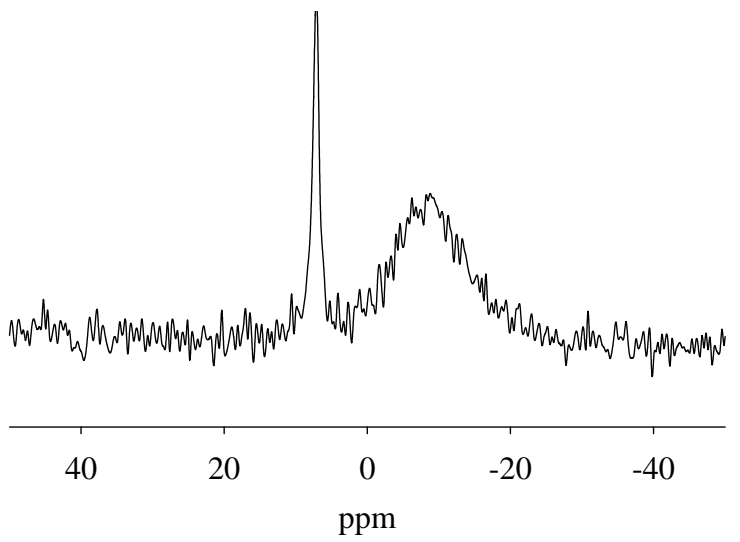

A

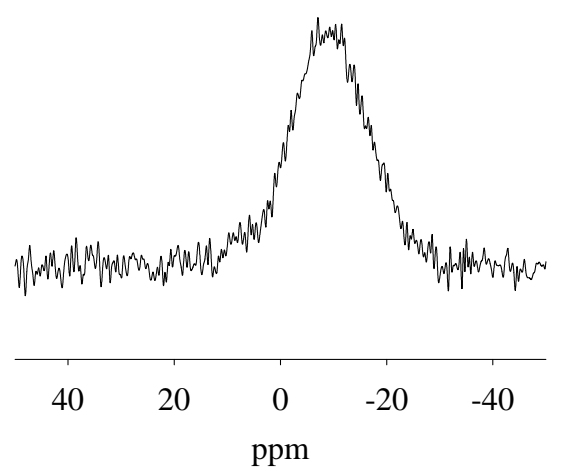

B

Figure 4. ${ }^{23} \mathrm{Na} \mathrm{NMR}$ of Sulfonated EVOH. A) as processed B) annealed at $120^{\circ} \mathrm{C}$ for $72 \mathrm{Hrs}$

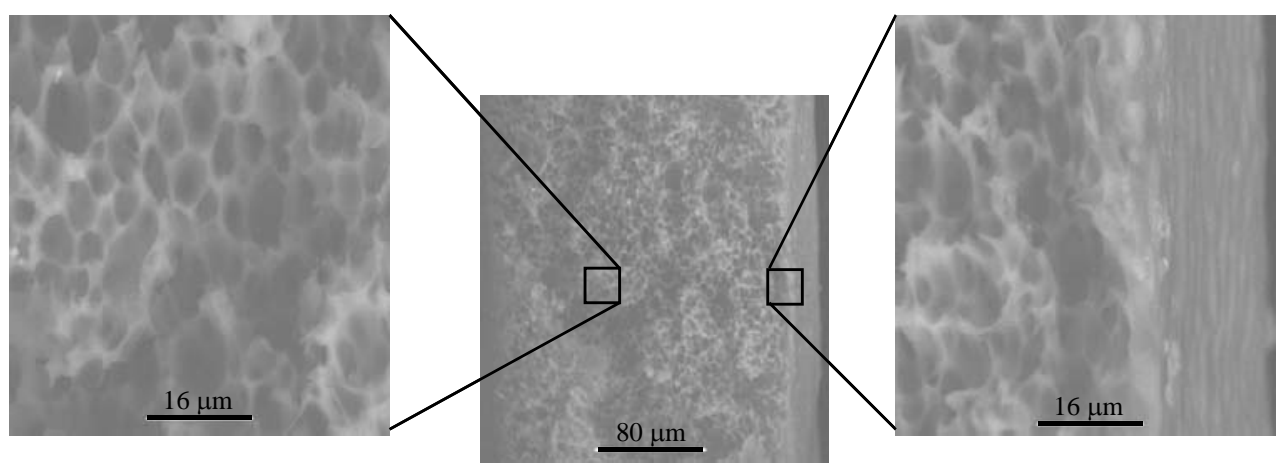

Figure 5. ESEM of an asymmetric polysulfone membrane cast from DMF 


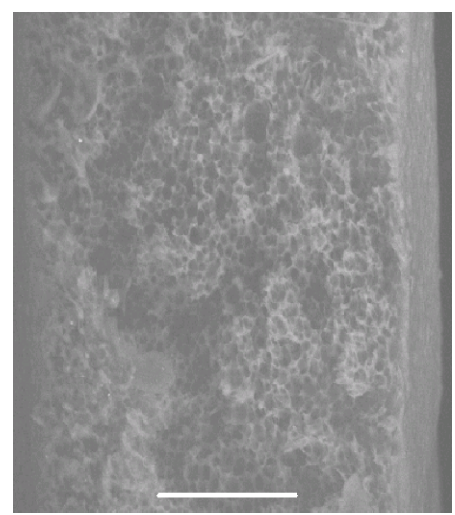

A

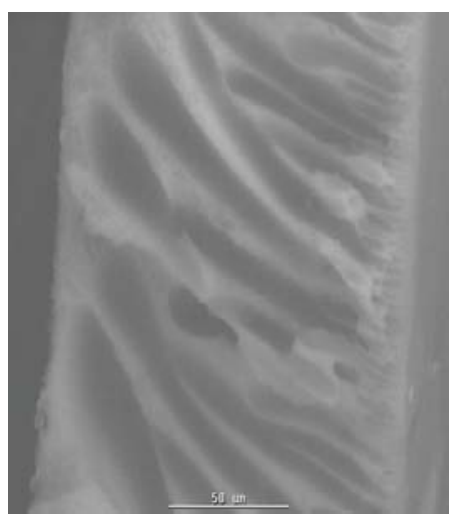

$\mathrm{B}$

Figure 6. ESEM of asymmetric polysulfone membrane cast from a) DMF and b) NMP

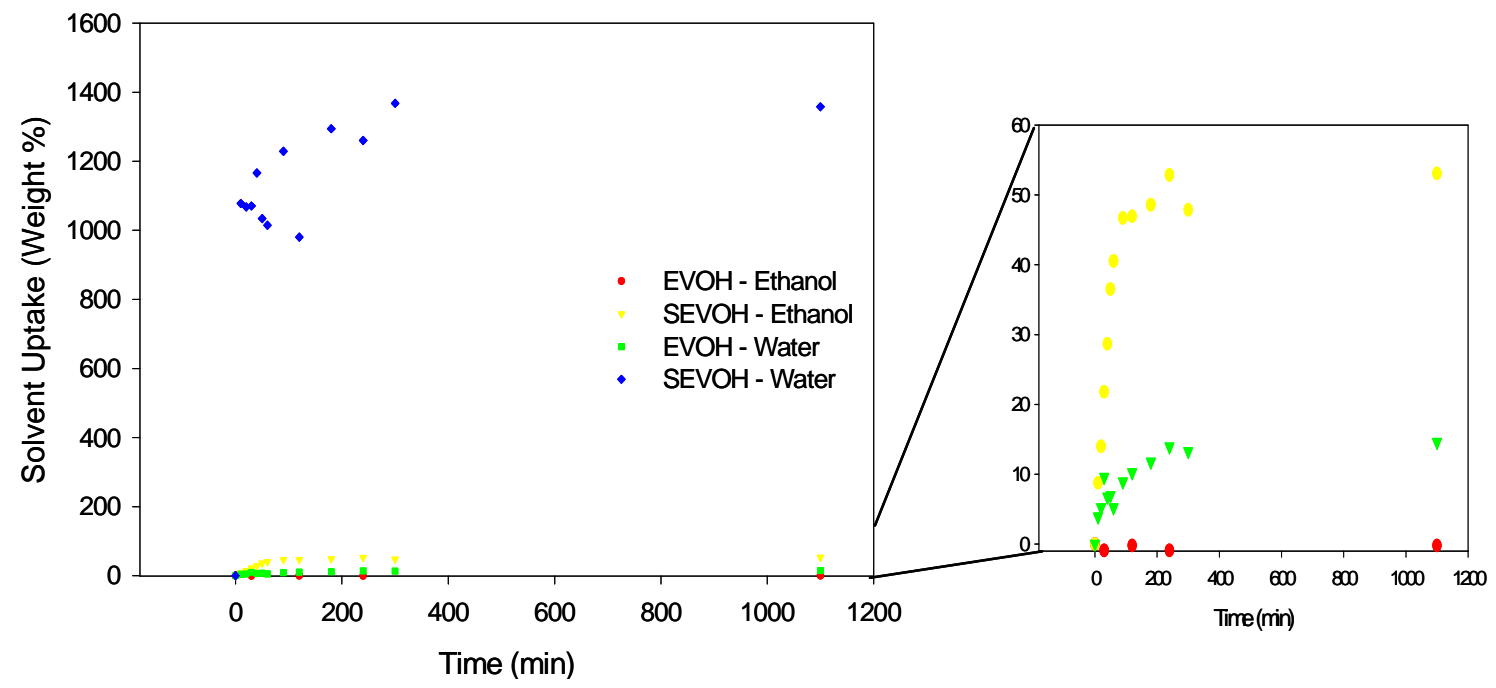

Figure 7. Differential swelling in EVOH and Sulfonated EVOH 


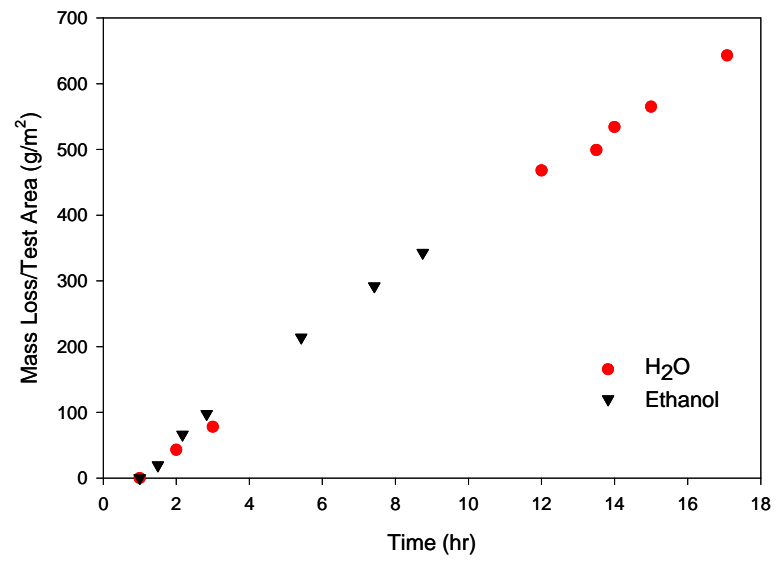

$$
\begin{aligned}
& P_{H 2 O}=3.16 \cdot 10^{-4} \frac{g}{m^{2} h r m m ~ H g} \\
& P_{E t O H}=1.62 \cdot 10^{-4} \frac{g}{m^{2} h r ~ m m ~ H g}
\end{aligned}
$$

Figure 8. Vapor permeation in Sulfonated EVOH

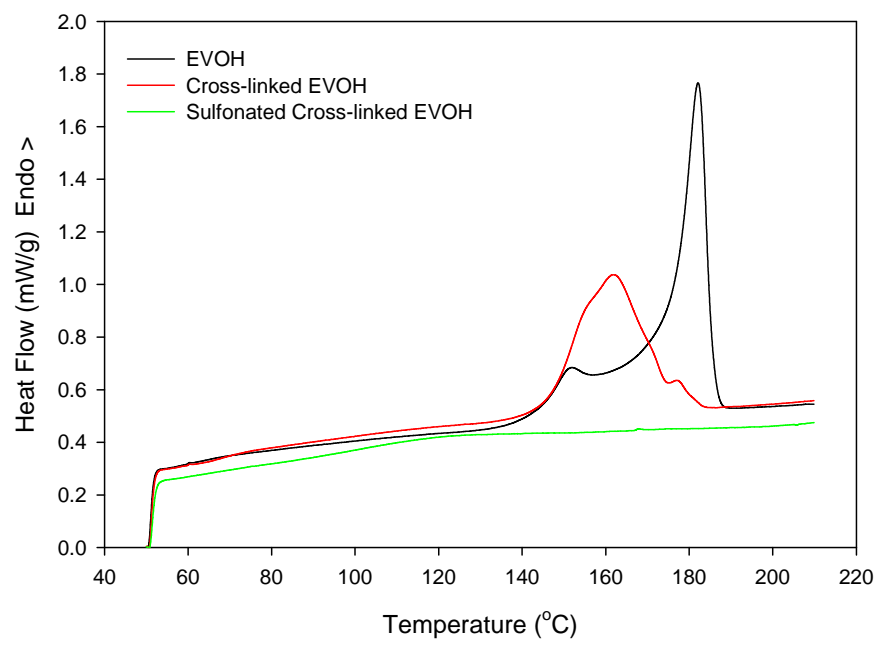

Figure 9. Crystallinity in EVOH and modified EVOH 


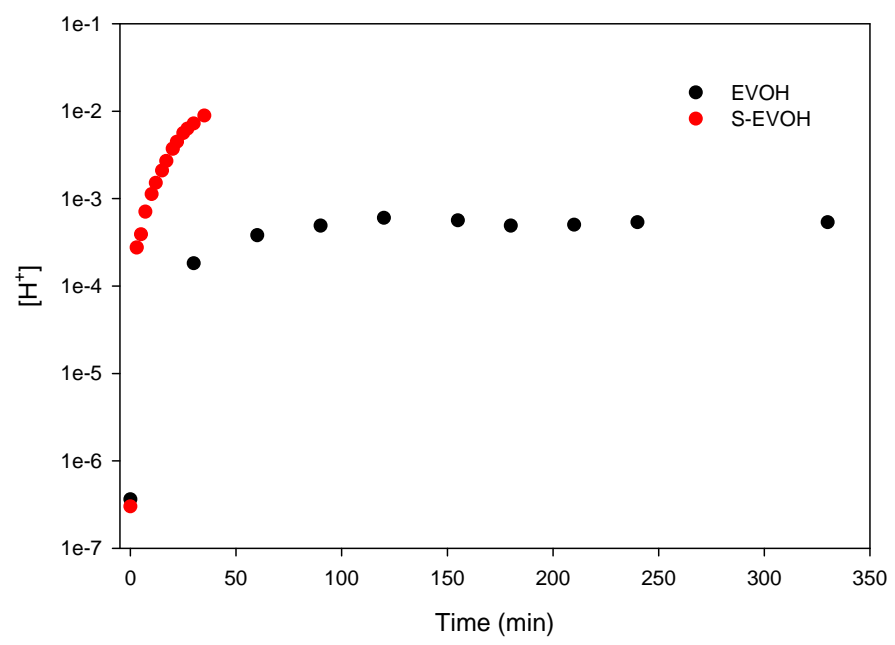

Figure 10. $\mathrm{H}^{+}$Permeation in EVOH and modified EVOH 


\section{ACID-SUGAR SEPARATION USING NOVEL MEMBRANE SYSTEMS Performer: University of Southern Mississippi \\ INTRODUCTION}

The highest fraction of the four major energy sources imported into the United States is petroleum [1]. The high cost of domestic exploration and production, as well as the high volumes of importation, are increasingly important factors affecting the US economy. Petroleum is primarily consumed in the transportation sector, in which there currently is no capacity to switch to another combustible fuel source [2]. For this reason interest has grown in the development of ethanol produced from cellulosic biomass as a large-scale transportation fuel. The use of ethanol as a renewable fuel source is desirable due to possible reductions in air pollution and in global climate changes, as well as providing a balance of trade and energy security. About a hundred areas in the United States do not meet air quality standards and $\mathrm{CO}_{2}$ emissions have been shown to be responsible for more than half the projected anthropically mediated climate change. The transportation sector is responsible for $27 \%$ of the total volume of $\mathrm{CO}_{2}$ emissions [1].

Ethanol can be used as a fuel either neat or in a mixture blended with small quantities of gasoline (e.g. E85, an $85 \%$ ethanol-15\% fuel gasoline blend). Currently in the United States, $7 \%$ of all gasoline contains ethanol derived from fermentation. Lowlevel ethanol-gasoline blends increase octane and fuel oxygen content. In Brazil about three million gallons of ethanol are used annually as neat fuel [3]. Hence, some of the most important car manufacturer's such as Fiat, Ford, General Motors and Volkswagen, have begun selling automobiles designed to operate on hydrous ethanol fuel [4]. Ethanol use in internal combustion engines has advantages such as higher thermal efficiency and power than combustion of gasoline, as well as a decrease in urban ozone levels and air toxicity [1]. Calculated air-quality improvements are around 0 to $15 \%$, depending on meteorological conditions, the source of pollutants, and the model used to determine the potential improvements [1]. Although this reduction may appear insignificant, ozone reduction is very difficult to achieve. Thus, these reductions have an important effect on air-quality improvements. 
The main feed stocks for ethanol production are hardwood, municipal solid waste and corn [1]. Corn is the primary raw material for the US fuel ethanol industry. Even though it may be a desirable source for low production levels, economic considerations show that production of ethanol from corn may not meet the significant transportation demand. Excess agricultural capacity in the United States suggests that the best source available for large-scale fuel ethanol might come from cropland waste, including all biomass sources.

\section{Ethanol Production as Fuel}

The conversion of cellulosic materials to ethanol involves two main steps: hydrolysis of the cellulose and fermentation of sugars. Hydrolysis refers to the breakdown of cellulosic materials into sugars using acids or cellulase enzymes. Fermentation refers to an enzymatically controlled anaerobic breakdown of glucose to yield ethanol. Hydrolysis can be achieved using either acids or enzymes. The projected selling prices for each case are comparable, although acid hydrolysis technologies have been used for many years in industry. Acid hydrolysis technologies are divided in two categories: concentrated acid hydrolysis and dilute acid hydrolysis.

Concentrated acid hydrolysis is based on concentrated acid decrystallization of cellulose followed by dilute acid hydrolysis to yield sugars. The technique to dissolve and hydrolyze cellulose using concentrated sulfuric acid in cotton followed by addition of water was reported as early as 1883 [5]. The concentrated acid breaks the hydrogen bonding between cellulose chains, leading to an amorphous state and forming a homogeneous gelatin with the acid. At this point, the susceptibility of cellulose to hydrolysis is increased. At low temperatures and dilution with water, complete and rapid hydrolysis of cellulose into glucose is achieved with very little degradation.

Dilute acid hydrolysis is a process in which hydrolysis occurs in two stages to account for the differences between hemicellulose and cellulose leading to maximum sugar yields [6]. Hemicellulose is any group of complex carbohydrates that surround the cellulose. In the first stage, hydrolysis of the more readily hydrolyzed hemicellulose is attained under mild conditions. In the second stage, hydrolysis of the more resistant cellulose fraction is accomplished. From each stage, the liquid hydrolyzates are recovered and subsequently fermented to yield alcohol. 
On the other hand, enzymatic hydrolysis is a process in which a cellulose enzyme is responsible for the hydrolysis to yield sugar. This process was first used to replace the cellulose acid hydrolysis step in the hydrolysis of wood to form ethanol. It was called separation hydrolysis and fermentation. Recently, a process has been introduced in which simultaneous saccharification and fermentation (SSF) is used to produce ethanol. Saccharification is the process of breaking a complex carbohydrate such as cellulose into simple sugars. Once the sugars are produced by the enzymes, the fermenting microbes convert them to ethanol. The key barrier for enzymatic processes to become economically feasible is the high cost of cellulase enzymes.

Concentrated acid hydrolysis has been shown to have more advantages than either dilute acid hydrolysis or enzymatic hydrolysis. Some of the advantages are that acid hydrolysis is effective on any substrate without pretreatment, may yield almost quantitative fermentable glucose, requires less dilute solutions for fermentation, may be completed within minutes and gives a valuable lignin residue as a by-product [7]. However, the main drawback of this kind of process is the high cost of acid recovery. Wright and coworkers [8] evaluated concentrated halogen acid hydrolysis processes for alcohol fuel production and reported the high cost of acid recovery. Numerous studies have focused on the use of volatile acids (i.e. halogen acids) in order to decrease the acid recovery cost.

Sulfuric acid has been used industrially in acid hydrolysis processes for ethanol fuel production even with the high cost of acid recovery. Sulfuric acid is a non volatile acid, thus it can not be recovered by distillation. Therefore, alternating technologies are necessary in order to recover the acid. Membrane technologies are increasingly being considered as a potential technique to be used for acid recovery due to lower energy cost. As an example, in the Hokkaido process developed by a Japanese company, the acid recovery was accomplished by diffusion dialysis using an anion exchange membrane [9].

\section{Membrane Technologies}

Membrane-based separation processes are an emerging technology which has gained industrial importance over the past 25 years due to its low energy consumption. The membrane design is crucial in the performance of the overall separation process. 
A membrane can be defined as a selective barrier between two phases [10]. A schematic representation of a membrane-based separation is shown in Figure 1. Separation is achieved due to the ability of the membrane to transport one component from the feed more readily than any other. Transport of one component through the membrane can be attained by various driving forces such as concentration gradients, pressure differences, temperature gradients and electrical forces [10]. Numerous membrane processes exist, but in our study we will focus on electrodialysis.

Electrodialysis is a membrane process in which charged species are separated from an electrolyte solution by an applied electrical current using a number of ion exchange membranes placed between a cathode and an anode. In contrast to common separation techniques such as distillation and freezing, an energy intensive phase change is not necessary, thus energy costs are reduced. The use of organic solvents often required in other selective transport techniques, such as liquid extraction, is avoided in electrodialysis. Another advantage is that electrodialysis is typically performed in mild temperature conditions and as a consequence, it is very attractive to food and pharmaceutical applications.

Researchers at North Carolina State University, Goldstein and coworkers, showed larger cost reductions using electrodialysis techniques [11] in acid recovery for concentrated acid hydrolysis of wood. The study reported that either diffusion dialysis or electrodialysis of sulfuric acid solutions are useful techniques for separation of the acid from sugars. Additionally, electrodialysis showed a 16 fold increase in acid flux compared to diffusion dialysis. They also predicted the cost of acid recovery per pound of glucose produced as a function of current efficiency and membrane service life at the optimal current density. Final acid recovery cost was estimated to be around $\$ 0.02$ and \$0.03 per lb in 1989.

\section{BACKGROUND}

\section{Ionomers As Membrane Materials}

lonomers are macromolecules containing a small number of ionic functionalities, usually less than 15 mole $\%$, along the polymer backbone [12]. Over the past few decades, interest in the field of ionomers has grown in both industrial applications and academic study due to their unique behavior and properties. The incorporation of ions 
has been shown to modify the mechanical properties of the material significantly [1315].

Different architectural structures of ionomers can be achieved synthetically such as monochelics, telechelics and side chain ionomers. Monochelics are the simplest type of ionomers in which a single ionic group is located on one chain end. Telechelics are the next level of complexity in which both end groups of the polymer chain contain ionic functionalities. In side chain ionomers, ionic groups are located at the end of side chains.

The strong Coulombic attractive forces between the ionic groups are responsible for the formation of aggregates consisting of several ion pairs termed "multiplets" [15]. Aggregates may act as physical crosslinks within the polymeric matrix, thus raising the glass transition temperature, tensile strength and melt viscosity of the polymer. The presence of "multiplets" reduces the mobility of the polymer chains in their vicinity, creating a loss in conformational entropy [14]. The presence of ionic aggregates has been proven with a wide range of techniques such as small angle $\mathrm{x}$-ray scattering (SAXS) [15-20] and neutron scattering (SANS) [20, 21], dynamical mechanical measurements [22-24], ${ }^{23} \mathrm{Na}$ NMR [25], viscosity properties [26, 27], infrared [28-30], Raman [31] nuclear magnetic resonance spectroscopy [32] and electron microscopy [34].

One of the most important applications for ionomers has been as membrane materials, especially in separation processes where an electrical potential difference is applied as the driving force, such as in electrodialysis. Additionally, they have been used in other separation processes such as nanofiltration [35], ultrafiltration [36], reverse osmosis [37], diffusion dialysis and pervaporation [38-42].

The presence of ionic functionalities generates strong interactions within the membrane and counter ions that are attracted to the fixed charges. Ion exchange membranes are capable of exchanging ions with an opposite charge to the fixed charge while repelling ions with the same charge. Ion exchange membranes may be divided in two types: cation exchange membranes (CEM) and anion exchange membranes (AEM). CEM contain fixed negative charges surrounded by positively charged ions. Conversely, an anion exchange membrane (AEM) has fixed positive charges that 
electrostatically bind negatively charged ions. Ions with an opposite charge to the fixed charge are referred to as counter ions. lons with the same charge to the fixed charge are referred as co-ions. Figure 2 shows a schematic representation of two ion exchange membranes.

There are a number of commercially available ionomers that have been used successfully in membrane separation processes such as perfluorosulfonates and perfluorocarboxylates. Examples of these types of ionomers are shown in Figure 3. These materials were initially prepared by DuPont, trademarked as Nafion $®$, and subsequently by other companies. These ionomers exhibit a structure like poly (tetrafluoroethylene) having excellent stability in thermal and electrochemical applications.

Gierke and Hsu proposed the Cluster-Network model for Nafion $\AA$, a polymer containing a polytetrafluoroethylene main chain and a perfluorosulfonate side chain, in which the polymeric ions and absorbed electrolyte phase-separate from the fluorocarbon backbone into spherical ionic clusters connected by short narrow, channels [44]. Ion transport properties and selectivity of hydrated membranes are well described by percolation and absolute rate theories [44]. Subsequent work by Gebel and coworkers expanded the study over a wide range of water contents. This study suggests that as the membrane is hydrated, the morphology shifts from one described by a local order model to a connected network of rod-like aggregates. Phase inversion occurs when the water content fraction is 0.5 and the polymer phase becomes a discontinuous phase of rod-like aggregates within a continuous water phase [45].

Many other morphologies can be observed in ionomeric membranes. Those morphologies can vary from rod-like aggregates as in the case of Nafion ${ }^{\circ}$ to homogeneously distributed ion pairs. Those morphologies arise depending on backbone chemistry such as rigidity, hydrophilicity etc.

\section{Preparation Of lon Exchange Membranes}

The preparation of an ion exchange membrane is quite difficult due to the necessity to achieve a well balance of properties. In general, the main characteristics of an ion exchange membrane are as follows [46]: 
- low electrical resistance, i.e. the permeability of an ion exchange membrane for the counter ions under the applied voltage should be as high as possible

- high ion permselectivity, i.e. an ion exchange membrane should be highly permeable to counter ions and should be impermeable to co-ions

- high mechanical strength and toughness, i.e an ion exchange membrane should be mechanically strong to be used in an electrodialysis set-up and possess a low degree of swelling or shrinking in the electrolytes solutions

- good chemical stability and good durability in practical use; i.e. an ion exchange membrane should be stable over a wide range of $\mathrm{pH}$ avoiding any cleavage of ionic moieties

- good dimensional stability; i.e. an ion exchange membrane should maintain dimensions to be able to fit on the electrodialysis membrane stack

- flatness and uniformity of properties over a large membrane area

- reasonable cost

Three main methods can be used to prepare a homogeneous ion exchange membrane. The first method consists of the polymerization or polycondensation of monomers in which at least one of the monomers contains a moiety that either is an ionic group, anioinic or cationic, or it may be converted into an ionic group. The second consists of the introduction of an ionic moiety into a preformed solid film. The last method consists of the introduction of an ionic moiety into a polymer followed by the dissolution of the polymer and the casting into a film to obtain the desired membrane.

One of the first ionic membranes was made by polycondensation of phenol with formaldehyde [47]. The reaction scheme is shown in Figure 4. After the polymerization, the solution was cast into a film. The membrane was obtained by solvent evaporation and excess monomer was removed by washing the membrane in deionized water.

Another commonly used technique is the polymerization of styrene and divinylbenzene and the subsequent addition of the ionic functionalities by either sulfonation or amination. Figure 5 shows the reaction scheme to obtain cation exchange and anion exchange membranes respectively.

Tokuyama Soda has patented a successful method to prepare an ion exchange membrane based on the above mentioned copolymerization of styrene and divinylbenzene [46]. This method is referred to as the paste method. The paste method is based on a paste that is coated onto a reinforcing cloth and wound with separating 
film onto a roll. Heat is applied to copolymerize the monomers and make the poly(vinyl chloride) gel in order to obtain the base membrane onto which ionic groups may be introduced. The paste is composed of a monomer with a functional group which allows the introduction of an ionic moiety, divinylbenzene, a radical polymerization initiator and a finely powdered poly(vinyl chloride) [46]. The reinforcing material is poly(vinyl chloride) [46]. These membranes are commercially available under the trademark Neosepta ${ }^{\circ}$.

Kang and coworkers [38]. introduced sulfonic acid groups into a previously preformed membrane of crosslinked poly(vinyl alcohol). The modified membrane showed higher flux and permselectivity compared to the simply crosslinked membrane. The main consideration using this method for preparing ion exchange membrane is that the monomer may either contain a cross-linking agent, or it may be grafted onto a film by radiation techniques.

Commercial polymers with aryl backbones such as polystyrene, polycarbonate, polysulfone and poly(phenylene oxide) were sulfonated by Khan and coworkers [48]. and studied as possible cation exchange membranes for fuel cells. The membranes were obtained by solution casting and solvent evaporation technique of the previously synthesized sulfonated polymers. Sulfonated polycarbonate showed a high ion exchange capacity, a good mechanical and thermal stability, a low sorption in methanol and a low cost and thus, appeared as a feasible alternative to commercially available Nafion ${ }^{2}$.

The properties of an ion exchange membrane are determined by the polymer matrix and the type and concentration of the fixed charges. The polymer matrix may present a hydrophobic or hydrophilic character which affects the equilibrium water uptake as well as the resultant mechanical, chemical and thermal stabilities of the membrane. The type and concentration of the fixed charges dictates the permselectivity and the electrical resistance of the membrane. However, the higher the degree of fixed charges the higher the water uptake for these polymers. Thus, crosslinking is essential to obtain membrane stability. The degree of crosslinking of the membrane increases the stability and selectivity of the membrane by reducing swelling. Higher degree of crosslinking leads to a higher electrical resistance of the membrane. The electrical resistance of the membrane may be modified by the number of fixed charges. A high 
charge density reduces the electrical resistance of the membrane and increases the selectivity. Due to high charge density, a higher degree of crosslinking is needed to overcome excessive swelling. A compromise between degree of crosslinking and fixed charge density is required to achieve optimal membrane selectivity, stability and electrical resistance. A good anionic membrane optimally combines low proton leakage, high limiting current density, low membrane voltage and high mechanical resistance [46].

\section{Principles of Electrodialysis}

Electrodialysis, an electrochemical membrane separation technique for ionic solutions combining electrolysis and dialysis, was first proposed in 1890 by Maigrot and Sabates [50]. This process has been extensively used in the separation and concentration of salts, acids and bases from aqueous solutions [51-57], the separation of monovalent ions from multivalent ions [58] and the separation of uncharged molecules from ionic molecules [7] [11]. Industrial applications of electrodialysis are the production of potable water from brackish water, the removal of metals from wastewater, the demineralization of whey, deacidification of fruit juices and the removal of organic acids from fermentation broth [10] [59].

In the electrodialysis process, a selective ionic transport is accomplished using ion exchange membranes as physical barriers where ions are either rejected or transported depending on the ionic charge and membrane charge. The main requirements for electrodialysis are directed towards maintaining a uniform distribution of solution flow and minimizing the electrical resistance and current leakage. Electrodialysis does not require a phase change, such as in distillation or freezing and thus, the requirements for strength and support of the membranes are less demanding. Another characteristic of this separation technique is that the use of an organic solvent, often required in separation techniques such as liquid extraction, is avoided. Also, this technique does not require harsh conditions and thus is very attractive in the food industry.

In electrodialysis charged species contained in the feed solution are separated from an electrolyte solution by an applied electrical current. The ionic separation is accomplished by a number of cation and anion exchange membranes placed in an 
alternating pattern between a cathode and an anode. The driving force for this type of separation is an electrical current applied to the electrodes. The membranes are separated by a spacer that serves to support the membranes, to give a passage for fluid flow and to decrease the thickness of the boundary layer adhering to the membrane.

Figure 6 shows the concept of electrodialysis in which $\mathrm{C}+$ refers to the positive ions and $\mathrm{A}$ - to the negative ions. When an ionic feed solution (e.g. sodium chloride solution) is pumped through the cell pairs in the presence of a direct current, the positive charged sodium ions migrate towards the cathode and the negatively charged chloride ions migrate towards anode. The CEM blocks the chloride ions and the AEM membrane blocks the sodium ions from further diffusion. The overall effect is that the ionic concentrations increase in alternating compartments accompanied by a simultaneous decrease in ionic concentration in the other compartments. Consequently, the feed solution is depleted of sodium chloride and the waste stream becomes concentrated in sodium chloride. Hence, the solution that is being diluted is called the diluate and the waste stream is referred to as concentrate. Hydrogen and hydroxyl ions are produced in the cathode, while oxygen and hydrogen ions are produced in the anode [60]. In order to maintain the conductivity throughout the system and prevent potentially corrosive ions from the feed solution to contact electrodes, an electrolyte rinse solution is pumped to the electrodes (i.e. $\mathrm{NaSO}_{4}$ solution).

\section{Principles Of Ion Exchange Membranes In Electrodialysis}

The distribution of ionic species is shown in Figure 7. As mentioned previously, the mobile ions with opposite charges to the fixed charges are called counter ions, while the mobile ions with the same charges are called co-ions. The exclusion of co-ions across an ionic membrane is based on the Donnan exclusion mechanism [10].

Consider the ionic distribution in a cation exchange membrane in contact with an electrolyte. The cation concentration in the membrane is higher than in the solution due to presence of negatively charged moieties. However, the concentration of anions in the solution is higher than in the membrane. Accounting only for diffusion mechanisms, the diffusion of cations into the solution and the diffusion of anions into the membrane would occur until the concentration difference equilibrates. However, diffusions of ions will create an electric field with a positive space charge in solution and a negative space 
charge in the membrane and thus, migration of negative species into the solution and positive species into the membrane would take place. An electrochemical equilibrium will be reached called the Donnan equilibrium in which the electric field balances the diffusional driving force of all the ions.

\section{Ion Transport Through lon Exchange Membranes}

The total transport of ions through an ion exchange membrane is due to three contributions, namely a convective component, a diffusive component and a migration component [10]. The flux of ions through a membrane may be defined as:

$$
J_{i}=v \cdot C_{i}-D_{i} \frac{d C_{i}}{d x}-\frac{z_{i} \cdot F \cdot C_{i} \cdot D_{i}}{R \cdot T} \cdot \frac{d \varphi}{d x}
$$

where $\mathrm{J}_{\mathrm{i}}$ is the flux of component $\mathrm{i}, \mathrm{v}$ the velocity of convective transport, $\mathrm{C}$ the concentration, $D$ the diffusion coefficient, $x$ the transport direction coordinate, $z$ the electrochemical valence, $\mathrm{F}$ the Faraday constant, $\mathrm{R}$ the gas constant, $\mathrm{T}$ the temperature and $\varphi$ the electrical valence. Since ion exchange membranes are non porous, the convective contribution can be neglected and thus the reduced equation results in the Nernst-Planck equation in which the ionic transport is expressed as a combination of diffusion and migration mechanisms.

The current density (i) passing through a membrane cell is determined according to Faraday's law as [10]:

$$
i=F \cdot \sum_{i=1}^{n} z_{i} \cdot J_{i}
$$

In the overall process of electrodialysis, a material balance can be written for the streams entering and exiting the membrane stack. The molar rate of a stream passing through the membrane stack can be calculated as [61]:

$$
m_{i}=F_{2}^{i} \cdot C_{2}^{i}-F_{1}^{i} \cdot C_{1}^{i}
$$

where the subscript 2 is the stream exiting the membrane stack and 1 is the stream entering, superscript $\mathrm{i}$ refers to either the concentrate or diluate stream, $\mathrm{F}$ is the volumetric flowrate, and $C$ is the concentration of the species being transported. When the electrodialysis process is operating in a batch mode the exiting streams are returned to the initial reservoir. The unsteady state material balance can be written as [61]: 


$$
\frac{d}{d t}\left(V^{i} \cdot C_{1}^{i}\right)=F_{1}^{i} \cdot C_{1}^{i}-F_{2}^{i} \cdot C_{2}^{i}
$$

Combining both expressions, an equation for the molar rate change in each reservoir can be obtained [61]:

$$
\dot{m}=-\frac{d}{d t}\left(V^{i} \cdot C_{1}^{i}\right)
$$

Transfer of ions from the rinse solution also takes place so that the overall balance is accounted for in the diluate, concentrate and rinse reservoirs [61]

$$
m_{r}+m_{c}+m_{f}^{\prime}=0
$$

Electrodialysis can be performed in two modes: constant-voltage or constant-current. At low voltages, the cell voltage and current within a membrane stack are related using Ohm's law:

$$
V=I \cdot R
$$

where $\mathrm{V}$ is the voltage, $\mathrm{I}$ is the electrical current and $\mathrm{R}$ is the resistance of the total membrane stack. The total resistance of the membrane stack is determined by the resistance of a cell pair multiplied by the number of cell pairs in the stack. The resistance of a cell part is determined by

$$
R_{c p}=R_{a m}+R_{c}+R_{c m}+R_{f}
$$

where $R_{c p}$ is the resistance of the one cell pair, $R_{a m}$ is the resistance of the anion exchange membrane, $R_{c}$ is the resistance of the concentrate compartment, $R_{c m}$ is the resistance of the cation exchange membrane and $R_{f}$ is the resistance of the feed.

The energy used to remove ions in an electrodialysis process is directly proportional to the total current flowing through the stack and the voltage drop between electrodes. Optimum processes should have as many membrane pairs as possible and as low voltage drop as possible.

The energy consumption in an electrodialysis can be calculated as [63]:

$$
E=I^{2} \cdot n \cdot R_{c p} \cdot t
$$

where $E$ is the energy consumption, $I$ is the electrical current through the stack, $R_{c p}$ is the resistance of a cell pair, $n$ is the number of cell pairs in the stack and $t$ is time. 
The electric current needed to deplete an electrolyte solution is directly proportional to the number of ions transferred from the feed stream to the concentrate stream through the ion exchange membrane and can be calculated as [64]:

$$
I=\frac{z \cdot F \cdot Q \cdot \Delta C}{\xi}
$$

where $F$ is the Faraday constant, $z$ is the electrochemical valence, $Q$ is the feed flow rate, $\Delta \mathrm{C}$ is the concentration difference between the feed and the concentrate stream and $\xi$ is the current utilization. The current utilization is controlled by the current efficiency of the electrodialysis cell and proportional to the number of cells in the stack.

The current efficiency of the stack is the ratio between the minimum theoretical charge required to transfer the ions calculated using the Faraday's law and the actual charge. For this reason, current efficiency is related to the performance of the process. The efficiency of the stack is calculated using the following equation:

$$
\eta=\frac{z \cdot F \cdot Q \cdot \Delta C}{n \cdot I}
$$

In electrodialysis, current efficiency is usually less than $100 \%$ mainly due to three factors [63]:

1. The co-ions are not completely excluded, especially at high feed concentrations

2. Water transport due to osmosis and solvated ions through the membrane

3. Partial electrical current leakage through the stack manifold

Water permeability of ion exchange membranes is highly dependent on the type and the concentration of the ions. This phenomenon is very important in concentrate electrolyte solutions and determines the upper concentration limit of the concentrate stream. Shahi and coworkers studied the transport properties of short chain aliphatic carboxylic acids in electrodialysis and mathematically described the water transport behavior under the influence of different current densities for monopolar ion exchange membranes. They reported that higher current densities lead to a higher water transport flux.

\section{OBJECTIVES}

Acid hydrolysis of wood is an effective process to produce ethanol. However, the primary drawback of this process is the high cost of the acid recovery. Electrodialysis 
using ion exchange membranes has been successfully used for separation and concentration of acid sugar mixtures [7] [11]. The work reported herein will examine a new ethylene vinyl alcohol-based ionomer as an ion exchange membrane in an electrodialysis process. It will focus on the synthesis and characterization of a new membrane using ethylene vinyl alcohol as the polymer matrix due to its intriguing balance of hydrophilic and hydrophobic monomers within the copolymer.

This work is divided into two main objectives. The first objective is the synthesis of a new ethylene vinyl alcohol-based ionomer membrane by sulfonation reaction followed by a crosslinking reaction. The second objective evaluates the use of this membrane in an electrodialysis process. Specific objectives include:

- Synthesis of sulfonated ethylene vinyl alcohol copolymer using propane sultone as the sulfonation agent.

- Establishing an optimum procedure for the sulfonation of ethylene vinyl alcoholbased ionomer.

- Developing a viable crosslinking reaction of sulfonated ethylene vinyl alcohol ionomer.

- Exploring different crosslinking agents and crosslinking methods.

- Establishing a crosslinking and casting procedure in order to obtain an ion exchange membrane with the required properties to be used in an electrodialysis set-up.

- Construction of an electrodialysis module to perform acid and acid-sugar separations.

- Comparison in the behavior of commercially available ion exchange membranes with the new EVOH based membranes synthesized in our laboratory.

- Establishing process-morphology relationships to improve the separation process.

\section{EXPERIMENTAL}

\section{Materials}

Ethylene vinyl alcohol copolymers (F104BTM ) were supplied by EVAL Company of America (Evalca). F104 grade materials, composed of 32 mole \% ethylene, were used throughout this study. Propane sultone (98\%), 1,4- terephthaldehyde (99\%), hexamethylene diisocyanate (98\%) and anhydrous dimethylsulfoxide were purchased from Aldrich and used without further purification. Potassium chloride, sodium chloride, sodium hydroxide and sodium sulfate were purchased from Fisher. 


\section{Membrane preparation}

\section{Synthesis of sulfonated ethylene vinyl alcohol ionomer}

The sulfonation of EVOH was achieved by grafting propanesultone onto the polymer in anhydrous DMSO. A solution of potassium tert-butoxide in anhydrous dimethylsulfoxide was prepared by stirring the mixture at $60^{\circ} \mathrm{C}$ under dry nitrogen for 1.5 hours. EVOH was dissolved in anhydrous dimethylsulfoxide at a concentration of $10 \mathrm{wt}$ $\%$ with vigorous stirring at $60^{\circ} \mathrm{C}$ in a round-bottom flask. The mixture was stirred under inert atmosphere until the polymer was completely dissolved. Potassium tert-butoxide dissolved in dimethylsulfoxide was added to the polymer solution in equimolar amounts to the hydroxyl groups to be reacted. After 1.5 hours, propane sultone was slowly added in the mixture to allow reaction with 10 mole $\%$ of total hydroxyl groups present in the mixture. The reaction was allowed to proceed for 16 hours at $85^{\circ} \mathrm{C}$. The solution containing the modified EVOH was then cooled back down to room temperature and stored without purification for further crosslinking reaction. Figure 8 shows the reaction scheme.

\section{Crosslinking reaction of sulfonated ethylene vinyl alcohol ionomer}

Due to the incorporation of the sulfonate group, the water insoluble EVOH became water soluble and thus, a crosslinking reaction was required to attain the necessary physical stability of the cast membrane. Two different crosslinking agents were studied: 1,4- terephthaldehyde (TPA) and hexamethylene diisocyanate (HDI). Crosslinking reactions of poly(vinyl alcohol) using dialdehydes, aldehydes with acid functional groups, and polysaccharides have been previously reported [66-68]. However, successful in situ concurrent crosslinking reaction and film casting were not achieved. The procedure was repeated using different amounts of crosslinker and at different casting temperatures to slow down the crosslinking reaction. However, gelation due to fast crosslinking reaction occurred after 30 minutes.

Toward the crosslinking of modified $\mathrm{EVOH}$, another synthetic route to obtain the desired physical stability of EVOH ionomer was explored. The new method consisted of the reaction between hydroxyl groups with diisocyanate to forma urethane linkage as shown in Figure 9. In this study, hexamethylene diisocyanate was chosen as the diisocyanate functionality. 
The required amount of the solution from the above step was added to a round bottom flask to obtain $4.5 \mathrm{~g}$ of modified $\mathrm{EVOH}$ and the total volume was kept constant at $100 \mathrm{~mL}$ by adding anhydrous dimethylsulfoxide. The solution was stirred vigorously at room temperature. Once homogenization was achieved, a quantity of hexamethylene diisocyanate was added drop wise in order to achieve the desired theoretical amount of crosslinking in the system. The desired theoretical amount of crosslinker is the percentage of crosslinking reagent required to chemically react with all the hydroxyl groups present in the polymer. The solution was well mixed for approximately 5 minutes. The entrapped air bubbles were removed by vacuum. Then, the film was casted on a mold under nitrogen atmosphere and allowed to react for 12 hours.

Finally, the membrane was dried by solvent evaporation at $110^{\circ} \mathrm{C}$. Membrane cleaning was accomplished by immersing the membranes in deionized water for four days to remove solvent and unreacted species.

The previously mentioned mold was made from aluminum and built in house with the dimensions that the electrodialysis cell required. The dimensions of the mold were $13.5 \times 20.5 \times 0.5 \mathrm{~cm}$. A commercially available fluoropolymer called Bytac $\AA$ was adhered the mold surface in order to obtain a non adherent mold surface for the membrane.

\section{Membrane Characterization}

\section{Attenuated Total Reflectance}

Attenuated total reflectance Fourier transform infrared (ATR-FTIR) was collected from a Digilab FTS-6000 imaging system. The following experimental conditions were used: a Ge single reflection crystal, an angle of incidence of 45 degrees and 200 co added scans. The spectra of unsulfonated, sulfonated and crosslinked membranes were scanned.

\section{Swelling Index}

The degree of swelling was determined as a function of the weight gain of a sample according to a previous procedure [69]. Strips of a dry membrane were equilibrated overnight in a $0.5 \mathrm{M} \mathrm{NaCl}$ solution to convert the membrane from the $\mathrm{K}+$ form to a $\mathrm{Na}+$ neutralized form. Then the membrane was immersed in deionized water at $25^{\circ} \mathrm{C}$. After 2 days, the membranes were taken out of the solution and surface water 
was removed carefully with absorbent paper before being weight to obtain the wet weight $\left(\mathrm{W}_{\text {wet }}\right)$. The sample was dried in a vacuum oven at $60 \mathrm{oC}$ until constant weight $\left(W_{\text {dry }}\right)$ was achieved. The degree of swelling was calculated with the following equation:

$$
\text { swelling }=\left(\frac{W_{\text {wet }}-W_{d r y}}{W_{d r y}}\right) * 100 \%
$$

In order to study the swelling behavior of the membranes in other solvents, ethanol sorption was also studied. Pieces of $4 \times 1 \mathrm{~cm}$ were used in quadruplet. The measurement method used is outlined as follows:

- Equilibrate the membrane piece in the desired solution for at least 8 hours.

- Measure the size and thickness of the membrane.

- Weigh the wet membrane after drying the membrane surface with absorbent paper.

- Drying of the membrane in a vacuum oven at $60 \circ \mathrm{C}$ for at least 24 hours

- Weigh the dry membrane

\section{lon Exchange Capacity}

The ion exchange capacity (IEC) of the novel membranes was determined by titration [69]. The ion exchange capacity (IEC) indicates the number of milequivalents of ions in one gram of the dry polymer. A membrane sample was converted to the acid form by immersion into a $1 \mathrm{M} \mathrm{HCl}$ solution for 24 hours. The sample was dipped in deionized water in order to remove sorbed acid. After, the sample was placed in a $2 \mathrm{M}$ $\mathrm{NaCl}$ solution to exchange the protons with $\mathrm{Na}+$ ions. The $\mathrm{NaCl}$, solution was renewed two times to assure complete exchange. The $\mathrm{NaCl}$ solutions containing the released protons were titrated with $\mathrm{NaOH} 0.2 \mathrm{~N}$. The ion exchange capacity was calculated as:

$$
I E C=\frac{n}{W_{d r y}}
$$

where $\mathrm{n}$ was the milequivalents of ions and Wdry was the mass of the dry membrane.

The degree of sulfonation (DS) is defined as the molar ratio of sulfonated units to the total basic units. The relationship between the degree of sulfonation and the ion exchange capacity is outlined as follows, 


$$
D S=\frac{I E C / 1000}{\left(1-M_{S E V O H} \cdot I E C / 1000\right)+I E C / 1000}
$$

where MSEVOH is the molecular weight of sulfonated EVOH ( $\mathrm{Na}$ form) and $\mathrm{MEVOH}$ is the molecular weight of EVOH.

\section{Permselectivity}

Permselectivity is the preferred permeation of one molecule through a polymer film with respect to other diffusing molecules in a mixture. The permselectivity of the membranes was determined by the static membrane potential method [69]. The experiment consisted of two cells separated by a membrane sample which contains different concentration of an electrolyte solution on each side. On either side of the solutions a calomel reference electrodes were placed to measure the potential difference across the membrane. On one side of the membrane a solution of $0.1 \mathrm{M} \mathrm{KCl}$ flowed through to the cell, while on the other side a $0.5 \mathrm{M} \mathrm{KCl}$ solution was pumped through the membrane. Both solutions were kept at room temperature. The permselectivity of the membrane is defined as the ratio of the measured potential difference ( $\Delta$ Vmeas) and the potential difference calculated assuming a $100 \%$ permselective membrane ( $\Delta$ Vcalc):

$$
\alpha=\frac{\Delta V_{\text {meas }}}{\Delta V_{\text {calc }}} * 100 \%
$$

The calculated potential difference is $36.9 \mathrm{mV}$ in the case of a 0.1 and a $0.5 \mathrm{M} \mathrm{KCl}$ solution using the Nerst equation:

$$
\Delta V_{\text {calc }}=\frac{R * T}{Z^{*} F} * \ln \left(\frac{C_{2} * \gamma_{2}}{C_{1} * \gamma_{1}}\right)
$$

in which $\mathrm{R}$ is the gas constant, $\mathrm{T}$ the temperature, $\mathrm{z}$ the electrochemical valence, $\mathrm{F}$ the Faraday constant, $\mathrm{C} 1$ and $\mathrm{C} 2$ the concentrations of the two electrolyte solutions and $\gamma 1$ and $\gamma 2$ the respective activity coefficients. Before an experiment, the membrane was equilibrated in a $0.1 \mathrm{M} \mathrm{KCl}$ solution for 24 hours.

Table 1 lists the membrane properties of the Neosepta ${ }$ ion exchange membranes used as a control for the electrodialysis cell. 


\section{Electrodialysis Set-up}

The electrodialysis module used in our experiments was assembled in house. The main components of the system are an electrodialysis cell, a power supply, an amp-meter connected to a $\mathrm{PC}$, a $\mathrm{pH}$ sensor, pumps and three solution reservoirs. Figure 10 shows the complete experimental set-up.

The electrodialysis cell was purchased from Electrosynthesis Corporation in Lancaster, New York. The electrodialysis cell was an ED-1 model high quality pilot scale cell for process development. It is built on a propylene cell body for superior chemical resistance and an individual membrane area of $0.01 \mathrm{~m} 2$. It can hold up to 20 membrane pairs which allows to a total membrane area of $0.2 \mathrm{~m} 2$. The cell is provided with four internal manifold flow paths; anolyte (anode electrode rinse), catholyte (cathode electrode rinse) and interleaved feed and concentrate loops. The cell stack includes turbulence promoting mesh spacers made from polypropylene and gaskets made from peroxide cured EPDM rubber. The gaskets assure the proper flow rates and the mesh is used in order to maximize mass transport within the cell. The anode was made from platinum plated titanium and the cathode was made from 316 stainless steel. Both electrodes are provided with external threaded electrical connections.

Double headed peristaltic pumps (Cole Parmer L/S Brushless variable-speed digital drive EW-07523-60) are used to pump the diluate, concentrate and rinse solutions. The flow rate for the feed and concentrate streams are $0.2 \mathrm{~L} / \mathrm{min}$, and 0.5 $\mathrm{L} / \mathrm{min}$ for the combined electrode rinse streams.

A power supply (Xantrex XHR 60-18) serves as source for direct current for the stack. It can provide a current in the range of 0-18 A and a voltage on the range of $0-60$ $V$. The ionic charge that passed through the stack is recorded by an amp-second meter (Rapid Model M-1000).

Sulfuric acid concentration was determined by titration in the diluate and concentrate compartments. Sugar content was measured by HPLC using a procedure developed in the NREL and the Hester Research Group [70]. The volume variations for both streams were measured by using graduate cylinders.

The membrane stack was comprised with an array of cation and anion exchange membranes. Two types of membrane configurations were studied. The first array of 
membranes consisted of an alternating array of cation and anion exchange membranes. This stack was constructed with two cation and three anion exchange membranes, with anion exchange membranes adjacent to the electrodes. The other array was composed of four cation and two anion exchange membranes, with cation exchange membranes adjacent to the electrodes. A $0.5 \mathrm{M}$ solution of $\mathrm{Na} 2 \mathrm{SO} 4$ was used as the electrode rinse solution. The feed solutions were acid and acid-sugar solutions. The acid feed solution was a $\mathrm{H} 2 \mathrm{SO} 4$ solution with a concentration of $0.5,0.8$ and $1 \mathrm{M}$. The content of sugar in the feed solutions was $1 \mathrm{wt} \%$. The predominant reaction at the anode with a neutral solution was oxygen production, while in the cathode it was the formation of hydrogen gas. The predominate electrode reactions are shown below,

Anode Reaction

$$
2 \mathrm{H} 2 \mathrm{O}(\mathrm{l}) \rightarrow \mathrm{O} 2(\mathrm{~g})+4 \mathrm{H}++4 \mathrm{e}-
$$

Cathode Reaction

$$
2 \mathrm{H} 2 \mathrm{O}(\mathrm{I})+2 \mathrm{e}-\rightarrow \mathrm{H} 2(\mathrm{~g})+2 \mathrm{OH}-
$$

The system was operated in a batch mode at a constant voltage. Two different voltages were explored, $6.0 \mathrm{~V}$ and $8.0 \mathrm{~V}$. Most of the experiments were carried out until the current dropped to around $0.1 \mathrm{~A}$. The remaining experiments were stopped when a constant volume of diluate was achieved.

\section{RESULTS AND DISCUSSION}

\section{ATR analysis}

ATR was used to verify the success in the modification of EVOH. A spectra comparing unsulfonated, sulfonated and crosslinked membranes of ethylene vinyl alcohol is presented in Figure 11. Hexamethylene diisocyanate was used as a crosslinker. The following experimental conditions were used: a Ge single reflection crystal, an angle of incidence of 45 degrees and 200 co added scans.

Examination of the spectra of pure EVOH and sulfonated EVOH showed the appearance of a peak at $1360 \mathrm{~cm}-1$ due to the asymmetric stretching of the $\mathrm{S}=\mathrm{O}$ bond in the sulfonated EVOH spectra. The symmetric vibration of this bond produces the characteristic split band of absorbance at 1150-1185 cm-1. The appearance of those peaks confirmed the introduction of the propane sultone into the EVOH. 
Comparing the sulfonated EVOH with the sulfonated crosslinked EVOH from the reaction of hydroxyl groups with hexamethylene diisocyanate, the appearance of two major peaks can be observed. The peak at $1640 \mathrm{~cm}-1$ is due to the $\mathrm{C}=0$ stretching vibration of the secondary amine (amide I band), while the peak at $1564 \mathrm{~cm}-1$ corresponds to the $\mathrm{N}-\mathrm{H}$ bending mixed with some $\mathrm{C}-\mathrm{N}$ stretching (amide II). The existence of those two peaks provides evidence for the presence of urea and urethane linkages in the membrane. The absence of a peak centered at $2277 \mathrm{~cm}-1$, corresponding to the asymmetric stretch of the $\mathrm{N}=\mathrm{C}=\mathrm{O}$ group, indicates that both functional groups of the diisocyanate had reacted.

\section{Swelling Studies}

Table 2 shows the equilibrium swelling indexes of water and ethanol of the pure copolymer $(\mathrm{EVOH})$, the sulfonated polymer (SEVOH) and the sulfonated crosslinked polymer (SEVOH_HDI).

Upon sulfonation of $\mathrm{EVOH}$, the membrane material becomes hydrophilic and thus the water uptake increases. Prior to sulfonation, the water uptake of $\mathrm{EVOH}$ was in the range of $13 \%$ showing a degree of hydrophilicity. The hydrophilicity of $\mathrm{EVOH}$ arises due to the presence of a 68 mole \% of vinyl alcohol in the copolymer. After sulfonation, the water uptake of modified EVOH membranes was drastically enhanced. A degree of sulfonation of $\mathrm{EVOH}$ in the order of 10 mole \% of the total hydroxyl groups showed a 30 fold increase in the water uptake of the membrane. Therefore, the crosslinking reaction of modified $\mathrm{EVOH}$ was essential in order to create a membrane with all the requirements previously mentioned for electrodialysis. The crosslinking reaction of the modified membrane with hexamethylene diisocyanate reduced the water uptake by almost $50 \%$.

Ethanol sorption values were lower compared to those in water. Hence, a preferential affinity towards water was observed. However, the same trend could be observed upon sulfonation and crosslinking. Sulfonated EVOH membranes had a higher methanol sorption while crosslinked membranes displayed a reduction in methanol sorption. 


\section{Ion Exchange Capacity And Degree Of Sulfonation}

The ion exchange capacity (IEC) indicates the number of mili-equivalents of ions in one gram of the dry polymer. The IEC of the EVOH modified membranes was $1.02 \pm 0.09$ meq./g measured by acid-base back titration using phenolphthalein as an indicator. The IEC of modified EVOH membranes was lower than the IEC for Tokuyama Neosepta cation exchange membranes but similar to Nafion ${ }^{\circledR}$. However, unlike Nafion ${ }^{\circledR}$ the cost of EVOH is very low.

The degree of sulfonation (DS) indicates the average number of sulfonic groups present in the sulfonated membrane. The degree of sulfonation obtained experimentally using the existing relationship with the ion exchange capacity was 0.042. The theoretical value for the degree of sulfonation was 0.068 . Thus, the calculated yield of the reaction was $62 \%$.

\section{Permselectivity}

The permselectivity value for the modified EVOH membranes was greater than $86 \%$. This value was smaller than the permselectivity value for the commercially available membranes.

\section{Electrodialysis Experiments}

Results of electrodialysis of acid solutions at $6.0 \mathrm{~V}$ applied voltage and using a membrane stack consisting of four cation exchange membranes (CMX) and two anion exchange membranes (AMX) are shown in Table 3. Under the influence of an applied voltage acting as the driving force, the ions move towards the electrodes. For the concentration of sulfuric acid, the sulfate ions $\left(\mathrm{SO}_{4}{ }^{2-}\right)$ pass through the anion exchange membranes and combine with the two protons $\left(\mathrm{H}^{+}\right)$that pass through the cation exchange membranes in the concentrate compartment. Therefore, as the results showed, the concentration of acid in the concentrate compartment increased while it decreased in the diluate compartment. It can be observed that as the initial acid concentration in the diluate compartment increased, the time for the experiment to be

finished and the number of coulombs of electricity that passed through the stack also increased. It was found that with the migration of ions from the diluate compartment to the concentrate compartment, water molecules were also transported. The water 
transport phenomenon was observed as volume changes. As it is shown in Table 4 there was an increase in the volume of the concentrate compartment while the volume of the diluate compartment decreased. Water molecules were transported as a result of electroosmosis, which is due to the flow of charged species through the membrane and osmosis due to a concentration gradient across the membrane. It can be observed by the experimental data that as the number of charged species transported through the membrane increased the water transport also increased. The number of charged species passing through the membrane is directly related to the initial diluate acid concentration.

The performance of the concentration of sulfuric acid by electrodialysis was calculated by the current efficiency and the difference between the final acid concentration and the initial acid concentration in the concentrate compartment $\left(\mathrm{C}_{\mathrm{c}}{ }^{\mathrm{t}}-\right.$ $\mathrm{C}_{\mathrm{c}}{ }^{\circ}$ ). Experimental data showed that as the initial acid concentration in the diluate compartment increases, the term $\left(\mathrm{C}_{\mathrm{C}}{ }^{\mathrm{t}}-\mathrm{C}_{\mathrm{c}}{ }^{\circ}\right)$ also increases, thus leading to a higher acid concentration in the concentrate compartment. Current efficiency is related to the number of moles of acid transported per Faraday of electricity passed per cell. The current efficiency values were calculated assuming that two electrons are required per mole of sulfuric acid, assuming that transport occurs as two protons and one sulfate anion (divalent). The values of current efficiency increased as the acid concentration in the diluate increased in spite of the higher number of coulombs that passed through the stack. Diffusion of acid molecules from the dilute compartment to the concentrate compartment due to a concentration gradient contributed to high apparent current efficiency.

Power consumption was calculated as the power consumed for the transportation of $1 \mathrm{~kg}$ of acid from the diluate compartment to the concentrate compartment. The required power will depend on the number of compartments within the stack, the membrane area and the acid to be recovered. The power consumption is affected by current efficiency and voltage. The power consumption was reduced progressively as the initial acid concentration in the diluate and the current efficiency increased. 
To study the effect of voltage on electrodialysis, experiments using the same membrane configuration stack but at $8.0 \mathrm{~V}$ applied voltage were performed as shown in Table 4. As observed before, the concentration of acid in the concentrate compartment increased while it decreased in the diluate compartment. The acid transport from the diluate compartment to the concentrate compartment also presented a water flux associated. The water flux increased as the initial acid concentration in the diluate compartment increased. However, at higher voltage, the time of the experiment is reduced and thus, the ions are transported more rapidly.

Comparing the performance of the concentration of sulfuric acid between the two voltages, the same degree of acid concentration is achieved. The term $\left.\left(\mathrm{C}_{c}{ }^{t}-\mathrm{C}_{c}{ }^{0}\right)\right)$ in both experiments remained almost unchanged. However, the current efficiency of the experiments carried out at $8.0 \mathrm{~V}$ applied voltage showed slightly higher values. This may be due to a rather reduced resistance of the electrodialysis unit. On the other hand, the current efficiency did not show the same trend as the experiments performed at a lower voltage. As the initial acid concentration in the diluate increased from 0.5 to $0.8 \mathrm{M}$ the current efficiency increased due to diffusion of acid from the diluate compartment to the concentrate compartment as expected for the results obtained before. Conversely, when the initial concentration in the diluate increased from 0.8 to $1.0 \mathrm{M}$ the current efficiency decreased. This effect can be attributed to an enhance back diffusion of acid from high concentrate compartment to low diluate compartment or concentration polarization at the membrane solution interphase leading to water splitting. The concentration difference between the concentrate compartment and the diluate compartment at a time $t$ also increased, showing an increase in back diffusion of acid.

The power consumption to run the experiments to concentrate sulfuric acid solutions showed a higher value for the trials performed at a higher voltage. The power consumption showed an approximately 1.2 fold enhancement by carrying the experiment at $8.0 \mathrm{~V}$ instead of $6.0 \mathrm{~V}$.

Another membrane stack configuration was studied and the results are shown in Table 5. This membrane stack configuration was composed by three anion exchange membranes and two cation exchange membranes displacing in an alternating manner. As a result of the electrodialysis, the concentration of acid in the concentrate 
compartment increased while it decreased in the diluate compartment. It can be observed a higher duration of the experiment and a higher number of coulombs of electricity passing through the stack as the initial acid concentration in the diluate increased. It was found that with the migration of ions from the diluate compartment to the concentrate compartment, water molecules were also transported due to electroosmosis.

Comparison with the results obtained with the previous membrane configuration showed a higher current efficiency due to higher $\left(\mathrm{C}_{\mathrm{c}}{ }^{\mathrm{t}}-\mathrm{C}_{\mathrm{c}}{ }^{0}\right)$. The values of current efficiency with the new configuration stack of membranes were around $70 \%$ while the current efficiencies for the previous configuration were in the range of $45 \%$. As a consequence, the power consumption for the transportation of $1 \mathrm{~kg}$ of acid from the diluate compartment to the concentrate compartment in the new configuration stack has lower values. This phenomenon can be explained due to the fact that using an anion exchange membranes in contact with the anode, positive electrode, would limit to some extent the transport of protons from the concentrate compartment to the rinse compartment. Hence, a higher final acid concentration in the concentration could be achieved.

In order to explore the separation of acid- sugar mixtures using electrodialysis, the membrane configuration consisted of three anion exchange membranes and two cation exchange membranes was utilized. This configuration was selected due to better performance showed previously.

Results of electrodialysis of acid-sugar solutions using commercially available Tokuyama ion exchange membranes are shown in Tables 6, 7, and 8. The experiments were run in a batch mode at $6.0 \mathrm{~V}$ applied potential. As the initial acid concentration in the diluate compartment increased, the number of transported ions through the membrane increased. Therefore, the time to conclude the experiment and the number of moles of acid transported per Faraday (96,500 coulombs) also increased. In the separation of acid from sugar, water transport was also observed with the diffusion of ions from the diluate compartment to the concentrate compartment. The water transported through the membranes increased as the initial acid concentration in the diluate compartment increased. 
The sugar content in the acid-sugar electrodialysis experiments remains the same in the diluate compartment as expected because sugar is a none electrically charged molecule. There is no sugar transport into the concentrate compartment.

The current efficiency showed a slight increase as the initial acid concentration increased from $0.5 \mathrm{M}$ to $0.8 \mathrm{M}$ since the difference between the final acid concentration and the initial acid concentration in the concentrate compartment increased. This can be attributed to a slightly reduced resistance that could compensate for the increased amount of electricity passing through the cell due to diffusion of acid from high diluate compartment to low concentrate compartment. On the other hand, a reduction in the current efficiency is observed as the initial acid concentration in the diluate increases from 0.8 to $1.0 \mathrm{M}$. This decrease can be attributed to back diffusion from high concentrate to low diluate.

Power consumption of acid removal using commercially available membranes decreased as the initial acid concentration of the diluate increases from 0.5 to $0.8 \mathrm{M}$ and increased as the initial acid concentration of the diluate increases from 0.8 to $1.0 \mathrm{M}$. The maximum value for power consumption was $2.36 \mathrm{kWh} / \mathrm{kg}$ of acid at an initial acid concentration of the diluate of $1.0 \mathrm{M}$. These results are in agreement with the values of current efficiency obtained for those experiments using only acid solutions.

Results of electrodialysis of acid-sugar solutions using EVOH modified membranes are shown in Tables 9, 10 and 11. The experiments were run in a batch mode at $6.0 \mathrm{~V}$ applied potential. Similar trends observed with commercially available membranes can be claimed using $\mathrm{EVOH}$ based membranes. As the initial acid concentration in the diluate increased, the number of transported ions through the membrane increased and thus, the time to conclude the experiment and the number of coulombs of electricity also increased. Water flux was also detected with the diffusion of ions from the diluate compartment to the concentrate compartment. However, due to the nature of the synthesized membranes the water transport was much more important. The water transported through the membranes increased as the initial acid concentration in the diluate compartment increased and was not only limited to the concentrate compartment. A water flux was also observed from the diluate compartment to the rinse compartment. 
The sugar content in the acid-sugar electrodialysis experiments remain mainly in the diluate compartment as expected because sugar is a none electrically charged molecule. However, the presence of a small amount of sugar in the concentrate compartment can be denoted in the experiment performed at the highest diluate initial acid concentration. This can be explained due to the degree of swelling and low permselectivity of the EVOH modified membrane that may allow the transport of some sugar molecules.

The current efficiency showed a dramatic decrease as the initial acid concentration increases. This can be explained due to the insufficient degree of crosslinking that would lead to a not enough permselectivity of the membranes. As the initial acid concentration in the diluate compartment increases the evidence of lack of permselectivity increases due to higher swelling of the membranes. The back diffusion mechanism becomes more important and leads to a decrease in current efficiency. However, the term $\left(\mathrm{C}_{\mathrm{c}}{ }^{\mathrm{t}}-\mathrm{C}_{\mathrm{c}}{ }^{\circ}\right)$ still increased as the initial acid concentration in the diluate increased. So, the desired sulfuric acid concentration by electrodialysis using EVOH modified membranes was still achieved.

Power consumption of acid removal using EVOH modified membranes increased as the initial acid concentration of the diluate increases. The power consumption is extremely dependent on current efficiency. For this reason, as the current efficiency decreases the power consumption increases. As the initial acid concentration increased, the more evident is the lack of high enough permselectivity of the EVOH modified membranes and the higher the back diffusion of acid. There is enhanced power consumption because of the existence of diffusion mechanism of ions in both directions, from diluate compartment to the concentrate compartment and the opposite. The maximum value for power consumption was $3.34 \mathrm{kwh} / \mathrm{kg}$ of acid at a initial acid concentration of the diluate of $1.0 \mathrm{M}$.

A comparison of the performance between commercially available Tokuyama ion exchange membranes and the modified EVOH membranes are shown in Tables 12 and 13. The initial and final acid concentrations in the diluate and concentrate compartment are in the same range in both cases. The term percent acid transferred represents the mole percent of the original acid in the diluate compartment that was transported 
through the membranes into the concentrate during electrodialysis. This term was similar in both sets of experiments. However, the experiments performed with the commercially available membranes showed a higher percentage of acid transferred even at the highest initial acid concentration in the diluate. In the case of EVOH modified membranes, the percentage of acid transferred was close to the one achieved by commercially available membranes at low acid concentration in the diluate. However, the percentage of acid transferred decreased when the initial acid concentration in the diluate was $1.0 \mathrm{M}$. The lower percentage of acid transferred in the $\mathrm{EVOH}$ based membranes is a consequence of higher degree of swelling compared to the commercial ones. Increasing the degree of crosslinking of EVOH based membranes would reduce the swelling and thus, the permselectivity of the membrane would increase.

The term referred to as $\Delta$ Final acid concentration is defined as the difference between the final acid concentrations in the diluate and concentrate compartments. Back diffusion of acid under osmotic pressures reduces this difference. For this reason, the experiments performed using the modified EVOH membranes showed a lower $\Delta$ Final acid concentration.

The current efficiencies for EVOH based membranes showed a lower value than the commercially available membranes. At low initial acid concentration in the diluate, the current efficiency of the EVOH modified membranes was just $5 \%$ less than commercial ones. At high initial diluate acid concentration, the differences between current efficiencies for EVOH modified membranes and commercial membranes increased drastically due to the lack in permselectivity of the novel membrane. The permselectivity of EVOH modified membranes should be increased to attain better results by increasing crosslinking density of the membranes and thus, reduce the swelling.

\section{SUMMARY}

In this project, the synthesis of novel ethylene vinyl alcohol membranes has been reported. A procedure has been established for the sulfonation and crosslinking reactions of ethylene vinyl alcohol copolymer. Sulfonation of ethylene vinyl alcohol was accomplished by using propane sultone as a sulfonation agent. The ion exchange 
capacity of the EVOH based membranes was 1.02 meq./g and the degree of sulfonation was in the range of 0.042 . The theoretical percentage of sulfonation was 0.068 and thus, the reaction yield was $62 \%$. In situ crosslinking and casting method to obtain a cation exchange membrane was outlined. Hexamethyelene diisocyanate was used as a crosslinker. The degree of crosslinking of the novel membranes results in a reduction of the water uptake by half.

Swelling and permselectivity studies have been carried out in order to characterize the modified EVOH membranes. Preferential swelling of these membranes in water versus ethanol has been shown. Hence, pervaporation of water-ethanol mixtures could be a possible new area of research for these membranes. A Permselectivity study reveals the lower permselectivity for EVOH based membranes compared to commercially available ones.

An electrodialysis module has been assembled in situ to study the separation of acid-sugar mixtures using either commercially available membranes or EVOH modified membranes. Two different membrane stack configurations have been explored. The optimum membrane stack configuration was composed by three anion exchange membranes and two cation exchange membranes. Studies with three different initial diluate acid concentrations, $0.5,0.8$ and $1.0 \mathrm{M}$, and a $1 \mathrm{wt} \%$ sugar content have been performed.

The behavior of commercially available membranes and modified EVOH membranes has been compared. At low initial acid concentration in the diluate, the performance of $\mathrm{EVOH}$ based membranes was close to the performance of commercially available membranes. However, as the initial acid concentration in the diluate compartment increases the novel membranes shows lower current efficiencies due to the lack of permselectivity. The insufficient permselectivity of the membranes is due to a high degree of swelling. Increasing crosslinking density would reduce water uptake and thus, increase the permselectivity of the membrane.

\section{FUTURE WORK}

Numerous research investigations may be done in order to achieve a better cation exchange membrane for electrodialysis. According to previous results, $\mathrm{EVOH}$ based membranes presents a lack of permselectivity due to excessive water uptake. 
This deficiency is more severe as the initial diluate acid concentration increases. Higher degree of permselectivity of these membranes needs to be achieved in order to attain better performance. The degree of permselectivity is controlled by the degree of crosslinking of the membrane. Thus, higher crosslinking density would increase permselectivity and reduce the swelling of the membrane.

In order to address the issues related with swelling of the membranes, hydrophilicity of the polymer matrix should be explored in detail. Inherent hydrophilicity of the membranes results in higher water uptake. The percentage of vinyl alcohol in the EVOH copolymer is responsible for the degree of hydrophilicity of the matrix. Thus, the reduction on the vinyl alcohol content of EVOH copolymer would reduce the hydrophilicity of the matrix so does the swelling degree.

Further studies dealing with the effect of the rigidity of the crosslinkers on the properties of the membranes should be considered. A complete understanding of how the crosslinker structure-influences the properties of the membranes is needed and would be obtain by examining and comparing different crosslinker structures. A less flexible crosslinker, such as isophorone diisocyanate, should reduce the swelling of the membranes and thus, increase permselectivity.

Electrodialysis module should be improved in order to obtain more reproducible data. Consistent flow rates should be achieved by a more accurate control of the peristaltic pump flow.

Other possible applications for EVOH based membranes should be investigated. Due to the observed preferential swelling of these membranes in water versus ethanol, pervaporation of water-ethanol mixtures should be considered as a possible new area of research. 


\section{REFERENCES}

1. Lynd, L. R.; Cushman, J. H.; Nichols, R. J., and Wyman, C. E. Science 1991, 251, 1318-1322.

2. DOE, R. Assessment of costs and benefits of flexible and alternative fuel use in the U.S. transportation sector, Progress Report One: Context and Analytical Framework; 1989.

3. Clean Fuels Rep.; 1990; p 82.

4. Ferchak, J. D., and Pye, E. K. Sol. Energy 1981, 26, 17.

5. Harris, E. E. In Advances in Carbohydrate Chemistry 1949, 4, 153-188.

6. Harris, J. F.; Baker, A. J.; Conner, A. H.; Jeffries, T. W.; Minor, J. L.; Patterson, R. C.; Scott, R. W.; Springer, E. L., and Zorba, J. General Technical Report FPL-45 1985.

7. Goldstein, I. S. Method for Recovering Acid from an Acid-Sugar Hydrolyzate. 5,244,553, 1991.

8. Wright, J. D.; Power, A. J., and Bergeron, P. W. In Solar Energy Research Institute 1985.

9. Oshima, M. Wood. Chemistry Process Engineering Aspects; Noyes Development: New York, 1965.

10. Mulder, M., Basic principles of membrane technology. 2nd ed.; Kluwer Academic Publisher: Dordrecht, 1996.

11. Goldstein, I. S.; Bayat-Makppi, F.; Sabharwal, H. S., and Singh, T. M. Applied Biochem. and Biotech. 1989, 20/21, 95-106.

12. Pineri, M., and Eisenberg, A., Structure and properties of ionomers. D. Reidel Publishing Co.: Dordrecht, Holland, 1987.

13. Eisenberg, A., and Kim, J. S., Introduction to lonomers. 1 ed.; New York, 1998.

14. Moore, R. B.; Gauthier, M.; Williams, C. E., and Eisenberg, A. Macromolecules 1992, 25, 5769-5773.Eisenberg, A.; Hird, B.; Moore, R. B. Macromolecules 1990, 23, 4098.

15. Eisenberg, A.; Hird, B., and Moore, R. B. Macromolecules 1990, 23, 4098.

16. Longwoth, R., In lonic Polymers. New York, 1975; Vol. Chapter 2.

17. Roche, E. J.; Stein, R. S., and MacKnight, W. J. J . Polym. Sci., Polym. Phys. 1980, 18, 1035.

18. Yarusso, D. J., and Cooper, S. L. Macromolecules 1983, 16, 1871-1880.

19. Moore, R. B.; Bittencourt, D.; Gauthier, M.; Williams, C. E.; and Eisenberg, A. Macromolecules 1991, 24, 1376-1382.

20. Gebel, G., and Moore, R. B. Macromolecules 2000, 33, 4850-4855. 
21. Earnest, T. R.; Higgins, J. S.; Handlin, D. L., and MacKnight, W. J. Macromolecules 1981, 14, 192.

22. Hird, B., and Eisenberg, A. Macromolecules 1992, 25, 6466-6474.

23. Kim, J. S.; Jackman, R. J., and Eisenberg, A. Macromolecules 1994, 27, 27892803.

24. Ma, X.; Sauer, J. A., and Hara, M. Macromolecules 1995, 28, 3953-3962.

25. O'Connel, E. M.; Root, T. W., and Cooper, S. L. Macromolecules 1994, 27, 58035810.

26. Lundberg, R. D., and Makowski, H. S. J. Polym. Sci., Polym. Phys. 1980, 18, 1821.

27. Hara, M., and Wu, J. L. Macromolecules 1986, 19, 2887.

28. Lowry, S. R., and Mauritz, K. A. J. Am. Chem. Soc. 1980, 102, 4665.

29. Mattera, V. D., and Risen, W. M. J. Polym. Sci., Polym. Phys. 1984, 22, 67.

30. Peiffer, D. G.; Hager, B. L.; Weiss, R. A.; Agarwal, P. K., and Lundberg, R. D. J. Polym. Sci., Polym. Phys. 1985, 23, 1869.

31. Neppel, A.; Butler, I. S., and Eisenberg, A. Canadian Journal of Chemistry 1979, 57, 2518.

32. Boyle, N. G.; McBrierty, V. J., and Eisenberg, A. Macromolecules 1983, $16,80$.

33. Handlin, D. L.; MacKnight, W. J., and Thomas, E. L. Macromolecules 1981, 14, 795.

34. Porat, Z.; Fryer, J. R.; Huxham, M., and Rubinstein, I. J. Physical Chem. 1995, 99, 4667.

35. Jegal, J., and Lee, K. H. Journal of Applied Polymer Science 1999, 72, 1755-1762.

36. Aptel, P.; Abidine, N.; Ivaldi, F., and Lafaille, J. P. Journal of Membrane Science 1985, 22, 199-215.

37. Yang, M. H. Polymer Testing 1995, 14, 415-424.

38. Kang, Y. S., and Park, H. C. Polymer(Korea) 1990, 14, (2), 178-184.

39. Sun, Y. M., and Huang, T. L. Journal of Membrane Science 1996, 110, 211-218.

40. Wu, L. G.; Zhu, C. L., and Liu, M. Journal of Membrane Science 1994, 90, 199205.

41. Rhim, J. W.; Yeom, C. K., and Kim, S. W. Journal of Applied Polymer Science 1998, 68.

42. Shi, Y.; Wang, X., and Chen, G. Journal of Applied Polymer Science 1996, 61, 1387-1394.

43. Visser, R. C., Electrodialytic recovery of acids and bases: multicomponent mass transfer description. 2001.

44. Hsu, W. Y., and Gierke, T. D. Journal of Membrane Science 1983, 13, 307. 
45. Gebel, G. Polymer 2000, 41, 5829.

46. Mizutani, Y. Journal of Membrane Science 1990, 49, 121-144.

47. Helfferich, F., Ion Exchange. McGraw-Hill: New York, 1962.

48. Khan, A. A.; Sridhar, S., and Smitha, B. Journal of Membrane Science 2003, 225, 63-76.

49. Cattoir, S.; Smets, D., and Rahier, A. Desalination 1999, 121, 123-130.

50. Shaposhnik, V. A., and Kesore, K. Journal of Membrane Science 1997, 136, 3539.

51. Demircioglu, M.; Kabay, N.; Kurucaovali, I., and Ersoz, E. Desalination 2002, 153, 329-333.

52. Ryabtsev, A. D.; Kotsupalo, N. P.; Titarenko, V. I.; Igumenov, I. K.; Gelfond, N. V.; Fedotova, N. E.; Morozova, N. B.; V.A., S., and Tibilov, A. S. Desalination 2001, 207-214.

53. Demircioglu, M.; Kabay, N.; Ersoz, E.; Kurucaovali, I.; Safak, C., and Gizli, N. Desalination 2001, 136, 317-323.

54. Gain, E.; Laborie, S.; Viers, P.; Rakib, M.; Hartmann, D., and Durand, G. Desalination 2002, 149, 337-342.

55. Novalic, S.; Kongbangkerd, T., and Kulbe, K. D. Journal of Membrane Science 2000, 166, 99-104.

56. Lee, E. G.; Moon, S. H.; Chang, Y. K.; Yoo, I. K., and Chang, H. N. Journal of Membrane Science 1998, 145, 53-66.

57. Pourcelly, G.; Tugas, I., and Gavach, C. Journal of Membrane Science 1993, 85, 195-204.

58. Vallois, C.; Sistat, P.; Roualdes, S., and Pourcelly, G. Journal of Membrane Science 2003, 216, 13-25.

59. Strathmann, H., Electrodialytic membrane processes and their practical application. Commet Adavances Course on Membrane Technology: Stuttgart, 1993.

60. Bungay, P. M.; Lonsdale, H. K., and Pinho, M. N. Synthetic Membranes: Science, Engineering and Applications. 1983.

61. Farrell, S.; Hesketh, R. P., and Slater, C. S. Chemical Engineering Education 2003.

62. Krol, J. J., Monopolar and bipolar ion exchange membranes: Mass transport limitations. Universiteit Twente, 1969.

63. Shaffer, L. H., and Mintz, M. S. Principles of Desalination. Spiegler, K.S. ed.; Academic Press: New York, 1966.

64. Strathmann, H. Journal of Membrane Science 1981, 9, 121-189.

65. Nagarale, R. K.; Gohil, G. S.; Shahi, V. K.; Trivedi, G. S.; Thampy, S. K., and Rangarajan, R. Desalination 2004, 171, 195-204. 
66. Sheibley, D. W.; Manzo, M. A., and Gonzalez-Sanabria, O. D. Journal of the Electrochemical Society 1983, 130, (2), 255-259.

67. Teramoto, N.; Saitoh, M.; Kuroiwa, J.; Shibata, M., and Yosomiya, R. Journal of Applied Polymer Science 2001, 82, 2273-2280.

68. Yeom, C. K., and Lee, K. H. Journal of Membrane Science 1996, 109, 257-265.

69. Strathmann, H., Electrodialysis, in Membrane Handbook. Van Nostrand Reinhold: New York, 1992; p 218-262.

70. Sluiter, A., Determination of sugars, byproducts, and degradation products in liquid fraction process samples; NREL, Department of Energy: 2004. 


\section{FIGURES}

Membrane

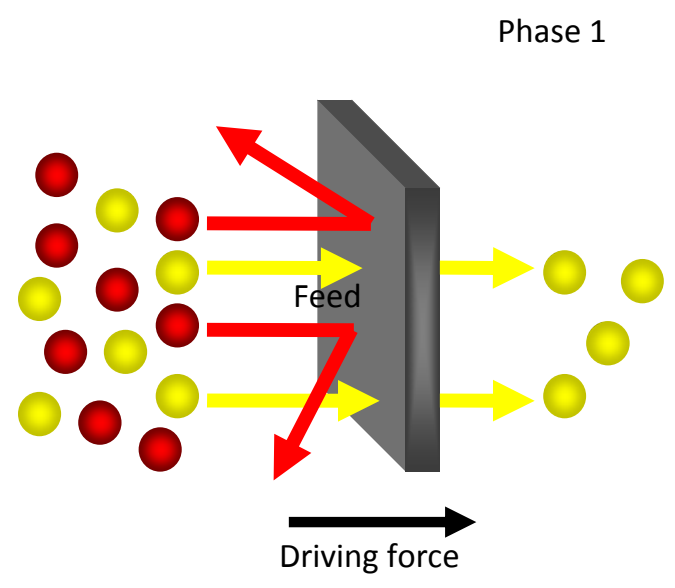

Permeatehse 1

$\Delta C, \Delta P, \Delta T, \Delta E$

Figure 1. Schematic representation of a biphasic system separated by a membrane.

Note: Phase 1 refers to the feed or upstream side and phase 2 refers to the permeate or downstream side.
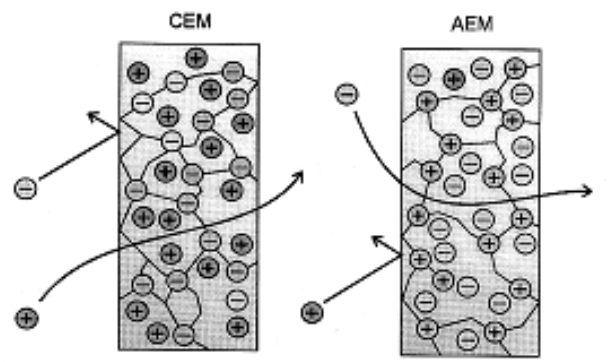

A $\mathrm{EFixed}$ charges

Q 6 Mobile charges

Figure 2. Representation of two types of ion exchange membranes: a cation exchange membrane membrane (left) and an anion exchange membrane (right) [43]. 
(a)

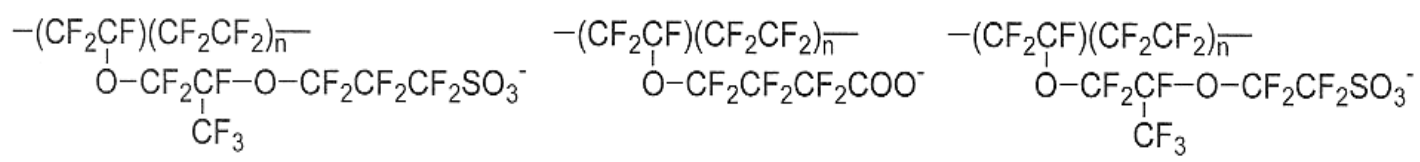

Figure 3. Examples of perfluorinated ionomers commercially available: Aciplex, (b) Flemion and (c) Nafion ${ }^{\circ}$.<smiles>O=S(=O)(O)c1ccc(O)cc1</smiles><smiles>Cc1cc(S(=O)(=O)O)cc(Cc2cc(S(=O)(=O)O)cc(Cc3cc(S(=O)(=O)O)cc(C)c3O)c2O)c1O</smiles>

Figure 4. Reaction scheme of the synthesis of an anion exchange membrane by polycondensation.<smiles>CCCCc1ccc(C(CCC)CCCC)cc1</smiles>

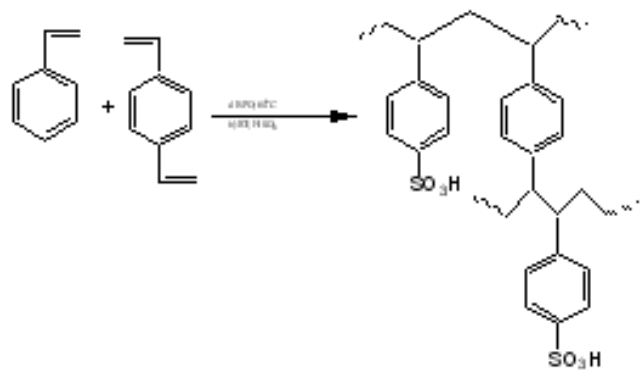

(a)

Figure 5. Reaction scheme to prepare an ion exchange membrane by the polymerization of styrene and divinylbenzene and subsequent (a) amination to obtain a cation exchange membrane or (b) sulfonation to obtain an anion exchange membrane. 


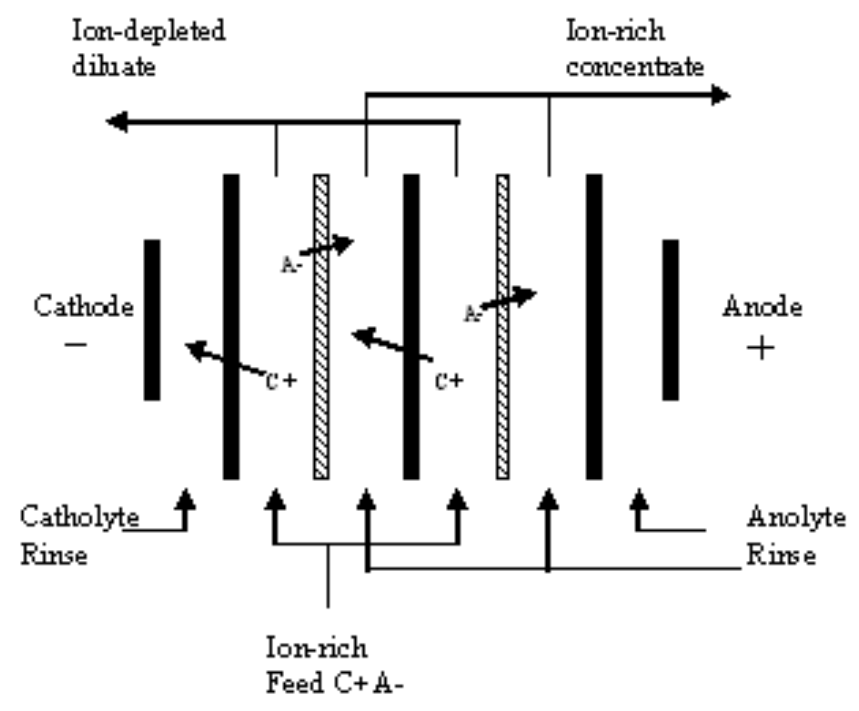

Figure 6. Scheme of the principles of electrodialysis [61]

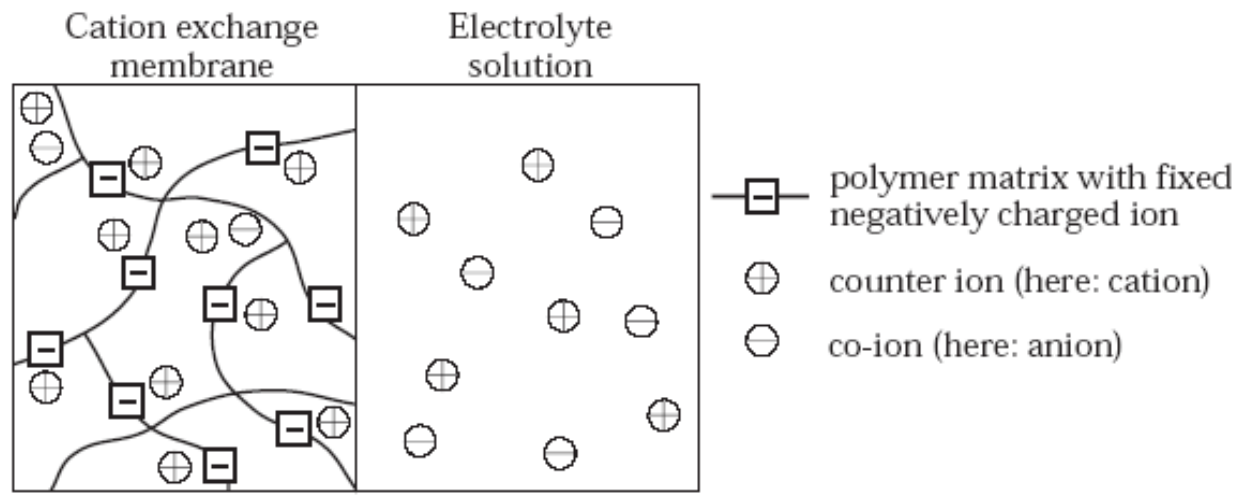

Figure 7. Principles of ion exclusion by ion exchange membranes [62]. 


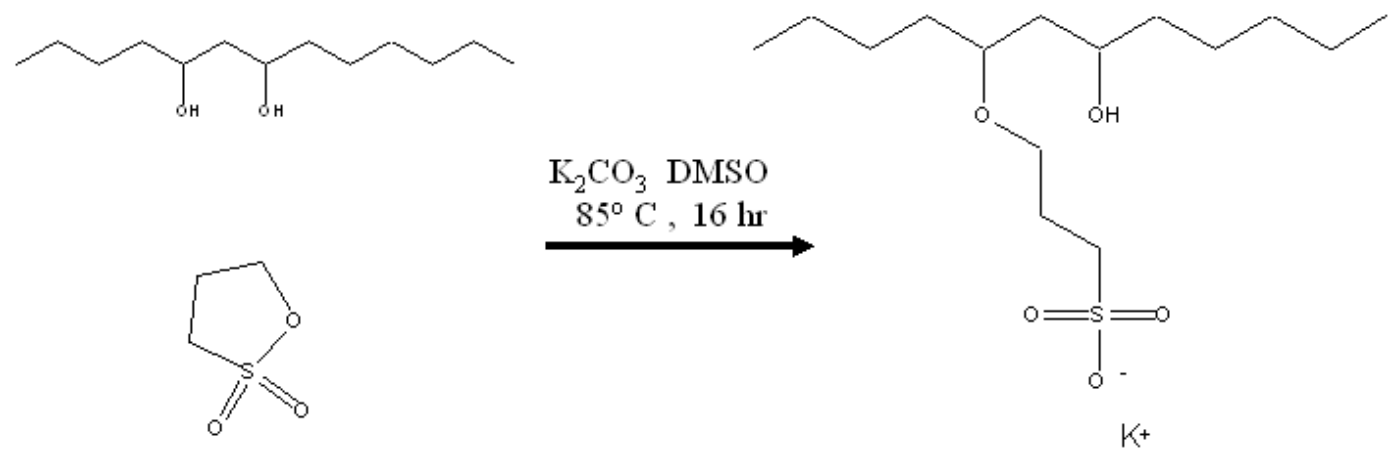

Figure 8. Reaction scheme of sulfonation of ethylene vinyl alcohol copolymer.

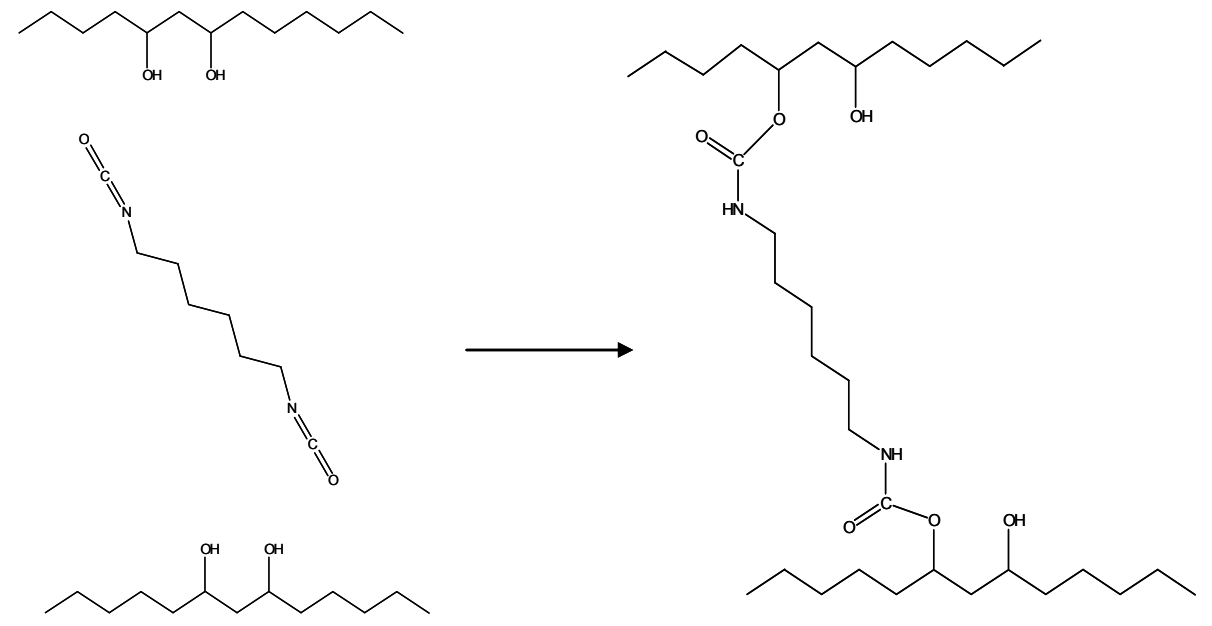

Figure 9. Reaction scheme of crosslinking of modified EVOH ionomer. 


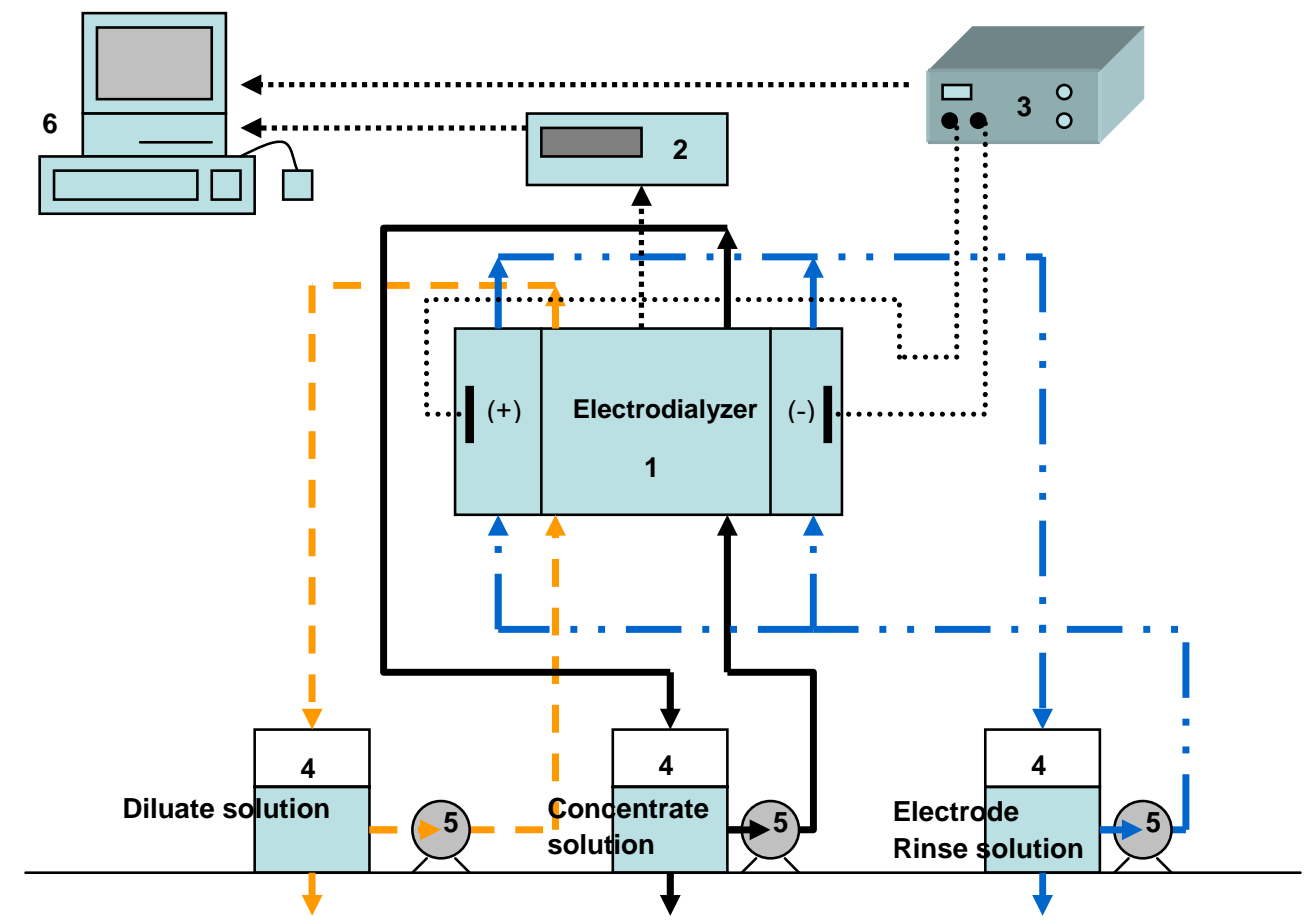

Figure 10. Experimental set-up of the electrodialysis module: (1) electrodialysis cell, (2) ampmeter, (3) power supply, (4) solution reservoirs, (5) peristaltic pumps, (6) PC. 


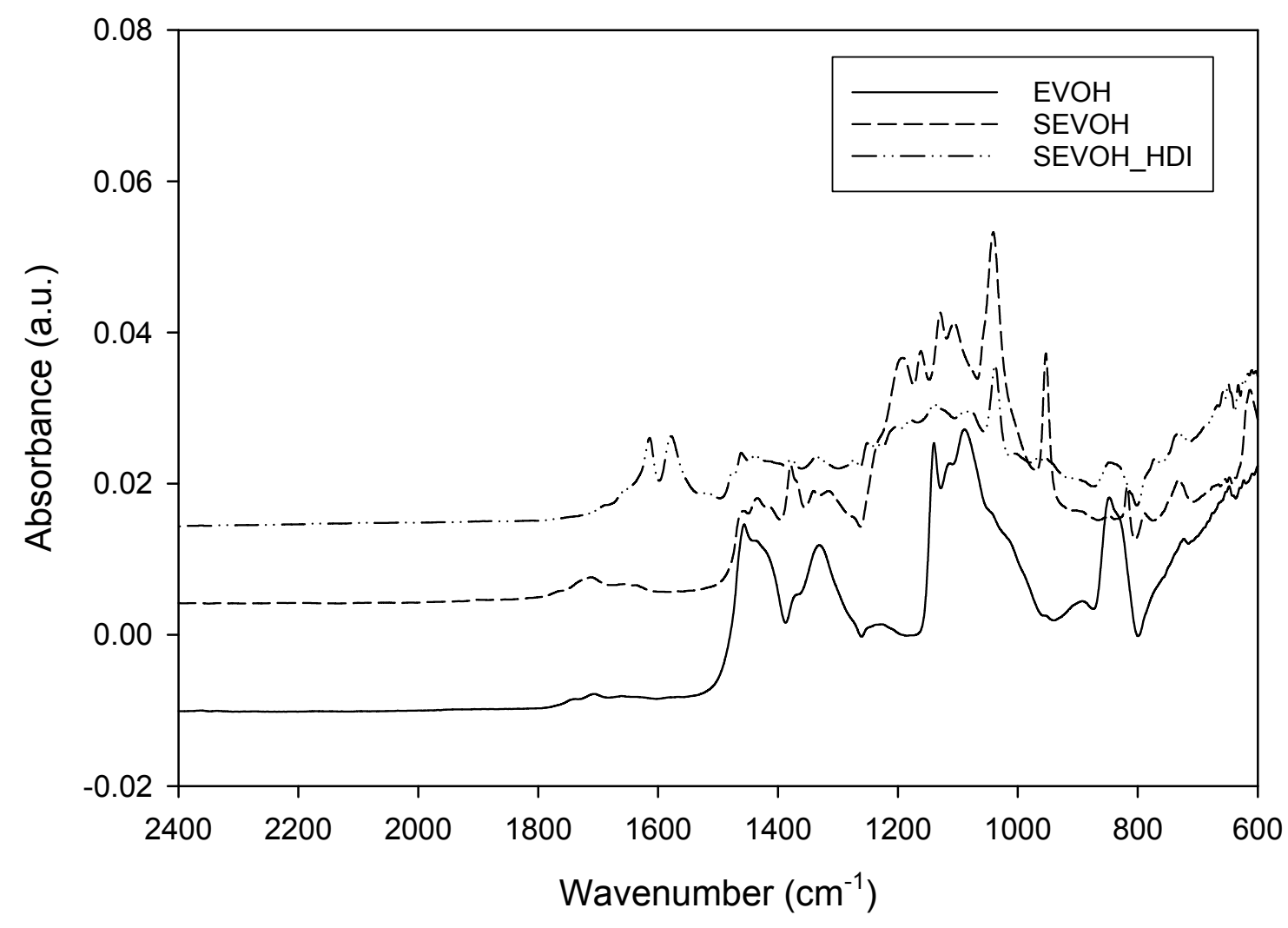

Figure 11. ATR spectra of: EVOH (-), sulfonated EVOH (----) and crosslinked sulfonated EVOH $(-\cdots \cdots-$.$) over the range 2000$ to $600 \mathrm{~cm}-1$. 


\section{TABLES}

Table 1. List of properties of Neosepta ${ }^{\circledR}$ ion exchange membrane.

\begin{tabular}{lll}
\hline Membrane & Property & Manufacturer data \\
CMX & Swelling (\%) & $25-30$ \\
& Exchange capacity (mequivl/g dry) & $1.5-1.8$ \\
& Electrical resistance $(\Omega \mathrm{cm} 2)$ & $2.5-3.5$ \\
& Permselectivity $(\%)$ & $>96$ \\
\hline AMX & Swelling (\%) & $25-30$ \\
& Exchange capacity (mequiv/ g dry) & $1.4-1.7$ \\
& Electrical resistance $(\Omega \mathrm{cm} 2)$ & $2.5-3.5$ \\
& Permselectivity $(\%)$ & $>96$ \\
\hline
\end{tabular}

Table 2. Sorption values for water and ethanol for the neat EVOH, the sulfonated EVOH (SEVOH) and the sulfonated crosslinked EVOH (EVOH_HDI).

\begin{tabular}{lll}
\hline Polymer & \multicolumn{2}{l}{$\begin{array}{l}\text { Swelling Index(\%) } \\
\text { Water }\end{array}$} \\
\cline { 2 - 3 } EVOH & $13 \pm 0.7$ & Ethanol \\
SEVOH & $399 \pm 58.7$ & $185 \pm 23$ \\
SEVOH_HDI & $152 \pm 3.4$ & $64 \pm 4$ \\
\hline
\end{tabular}


Table 3. Results of electrodialysing of sulfuric acid solutions at $6.0 \mathrm{~V}$ applied potential using commercially available membranes.

\begin{tabular}{|c|c|c|c|}
\hline \multirow[t]{2}{*}{$\begin{array}{l}\text { Cell } \\
\text { Stack: } \quad \text { C/C/A/C/A/C }\end{array}$} & \multicolumn{3}{|c|}{ Time (min) } \\
\hline & 120 & 160 & 167 \\
\hline $\mathrm{Q}$ (Coulomb) & 24966 & 41773 & 49364 \\
\hline \multicolumn{4}{|l|}{ Concentrate stream: } \\
\hline Initial concentration (M) & 0.11 & 0.11 & 0.10 \\
\hline Final concentration (M) & 0.35 & 0.53 & 0.62 \\
\hline Initial volume (ml) & 400 & 400 & 400 \\
\hline Final volume (ml) & 440 & 470 & 480 \\
\hline$\left(\mathrm{C}_{\mathrm{c}}^{\mathrm{t}}-\mathrm{C}_{\mathrm{c}}{ }^{\circ}\right)(\mathrm{M})$ & 0.24 & 0.42 & 0.51 \\
\hline \multicolumn{4}{|l|}{ Dilute stream: } \\
\hline Initial concentration (M) & 0.57 & 0.80 & 0.98 \\
\hline Final concentration (M) & 0.03 & 0.01 & 0.02 \\
\hline Initial volume $(\mathrm{ml})$ & 400 & 400 & 400 \\
\hline Final volume (ml) & 360 & 330 & 320 \\
\hline $\begin{array}{l}\text { Power consumption } \\
\text { (kWh/kg of acid removal) }\end{array}$ & 3.84 & 3.47 & 3.30 \\
\hline Current Efficiency\% & 42.74 & 47.21 & 49.63 \\
\hline
\end{tabular}


Table 4. Results of electrodialysing of sulfuric acid solutions at 8.0V applied potential using commercially available membranes.

\begin{tabular}{|c|c|c|c|}
\hline \multirow[t]{3}{*}{$\mathrm{C} / \mathrm{C} / \mathrm{A} / \mathrm{C} / \mathrm{A} / \mathrm{C}$} & \multirow{2}{*}{\multicolumn{3}{|c|}{ Time (min) }} \\
\hline & & & \\
\hline & 55 & 78 & 100 \\
\hline Q (Coulomb) & 22934 & 35205 & 46666 \\
\hline \multicolumn{4}{|l|}{ Concentrate stream: } \\
\hline Initial concentration (M) & 0.11 & 0.11 & 0.11 \\
\hline Final concentration (M) & 0.34 & 0.51 & 0.60 \\
\hline Initial volume (ml) & 400 & 400 & 400 \\
\hline Final volume (ml) & 440 & 460 & 480 \\
\hline$\left(\mathrm{C}_{\mathrm{c}}{ }^{\mathrm{t}}-\mathrm{C}_{\mathrm{c}}{ }^{\circ}\right)(\mathrm{M})$ & 0.23 & 0.40 & 0.49 \\
\hline \multicolumn{4}{|l|}{ Dilute stream: } \\
\hline Initial concentration (M) & 0.57 & 0.80 & 0.98 \\
\hline Final concentration (M) & 0.21 & 0.02 & 0.05 \\
\hline Initial volume (ml) & 400 & 400 & 400 \\
\hline Final volume $(\mathrm{ml})$ & 360 & 340 & 320 \\
\hline $\begin{array}{l}\text { Power consumption } \\
\text { (kWh/kg of acid removal) }\end{array}$ & 4.89 & 4.22 & 4.36 \\
\hline Current Efficiency\% & 44.64 & 51.84 & 50.10 \\
\hline
\end{tabular}


Table 5. Results of electrodialysing of sulfuric acid solutions at $6.0 \mathrm{~V}$ applied potential using commercially available membranes.

\begin{tabular}{|c|c|c|c|}
\hline \multirow[t]{3}{*}{$\begin{array}{l}\text { Cell } \\
\text { Stack: }\end{array}$} & \multirow{2}{*}{\multicolumn{3}{|c|}{ Time (min) }} \\
\hline & & & \\
\hline & 94 & 115 & 139 \\
\hline $\mathrm{Q}$ (Coulomb) & 26083 & 36291 & 44532 \\
\hline \multicolumn{4}{|l|}{ Concentrate stream: } \\
\hline Initial concentration (M) & 0.11 & 0.11 & 0.11 \\
\hline Final concentration (M) & 0.53 & 0.67 & 0.78 \\
\hline Initial volume (ml) & 400 & 400 & 400 \\
\hline Final volume (ml) & 440 & 470 & 480 \\
\hline$\left(\mathrm{C}_{\mathrm{c}}^{\mathrm{t}}-\mathrm{C}_{\mathrm{c}}{ }^{\mathrm{o}}\right)(\mathrm{M})$ & 0.42 & 0.56 & 0.68 \\
\hline \multicolumn{4}{|l|}{ Dilute stream: } \\
\hline Initial concentration (M) & 0.57 & 0.79 & 0.98 \\
\hline Final concentration (M) & 0.01 & 0.01 & 0.008 \\
\hline Initial volume (ml) & 400 & 400 & 400 \\
\hline Final volume (ml) & 360 & 330 & 330 \\
\hline $\begin{array}{l}\text { Power consumption } \\
\text { (kWh/kg of acid removal) }\end{array}$ & 2.33 & 2.26 & 2.37 \\
\hline Current Efficiency\% & 70.23 & 72.45 & 69.19 \\
\hline
\end{tabular}


Table 6. Results of electrodialysing of acid-sugar solutions at $6.0 \mathrm{~V}$ applied potential using commercially available membranes.

\begin{tabular}{|c|c|c|c|}
\hline $\begin{array}{l}\text { Cell } \\
\text { Stack: }\end{array}$ & $\mathrm{A} / \mathrm{C} / \mathrm{A} / \mathrm{C} / \mathrm{A}$ & & \\
\hline Current Density (A/m2) & & 61.36 & \\
\hline Time (min) & & 97 & \\
\hline Q (Coulomb) & & 21427 & \\
\hline \multicolumn{4}{|l|}{ Concentrate stream: } \\
\hline & & Acid conc. (M) & Sugar conc.(\%) \\
\hline Initial concentration & & 0.11 & 0.00 \\
\hline Final concentration & & 0.45 & 0.02 \\
\hline Initial volume (ml) & & 400 & \\
\hline Final volume (ml) & & 440 & \\
\hline Delta concentration (M) & & 0.35 & \\
\hline \multicolumn{4}{|l|}{ Dilute stream: } \\
\hline & & Acid conc. (M) & Sugar conc. $(\%)$ \\
\hline Initial concentration & & 0.50 & 1.09 \\
\hline Final concentration & & 0.005 & 1.06 \\
\hline Initial volume (ml) & & 400 & \\
\hline Final volume (ml) & & 360 & \\
\hline $\begin{array}{l}\text { Power consumption } \\
\text { (kWh/kg of acid removal) }\end{array}$ & & 2.33 & \\
\hline Current Efficiency\% & & 70.36 & \\
\hline
\end{tabular}


Table 7. Results of electrodialysing of acid-sugar solutions at $6.0 \mathrm{~V}$ applied potential using commercially available membranes.

\begin{tabular}{|c|c|c|c|}
\hline $\begin{array}{l}\text { Cell } \\
\text { Stack: }\end{array}$ & $\mathrm{A} / \mathrm{C} / \mathrm{A} / \mathrm{C} / \mathrm{A}$ & & \\
\hline Current Density $(\mathrm{A} / \mathrm{m} 2)$ & & 81.35 & \\
\hline Time (min) & & 116 & \\
\hline $\mathrm{Q}$ (Coulomb) & & 33971 & \\
\hline \multicolumn{4}{|l|}{ Concentrate stream: } \\
\hline & & Acid conc. (M) & Sugar conc. $(\%)$ \\
\hline Initial concentration & & 0.11 & 0.00 \\
\hline Final concentration & & 0.66 & 0.04 \\
\hline Initial volume (ml) & & 400 & \\
\hline Final volume (ml) & & 460 & \\
\hline Delta concentration (M) & & 0.55 & \\
\hline \multicolumn{4}{|l|}{ Dilute stream: } \\
\hline & & Acid conc. (M) & Sugar conc. $(\%)$ \\
\hline Initial concentration & & 0.79 & 1.04 \\
\hline Final concentration & & 0.01 & 1.14 \\
\hline Initial volume (ml) & & 400 & \\
\hline Final volume (ml) & & 340 & \\
\hline $\begin{array}{l}\text { Power consumption } \\
\text { (kWh/kg of acid removal) }\end{array}$ & & 2.22 & \\
\hline Current Efficiency\% & & 73.78 & \\
\hline
\end{tabular}


Table 8. Results of electrodialysing of acid-sugar solutions at 6.0V applied potential using commercially available membranes.

\begin{tabular}{|c|c|c|c|}
\hline $\begin{array}{l}\text { Cell } \\
\text { Stack: }\end{array}$ & $\mathrm{A} / \mathrm{C} / \mathrm{A} / \mathrm{C} / \mathrm{A}$ & & \\
\hline Current Density (A/m2) & & 117.66 & \\
\hline Time (min) & & 104 & \\
\hline $\mathrm{Q}$ (Coulomb) & & 44052 & \\
\hline \multicolumn{4}{|l|}{ Concentrate stream: } \\
\hline & & Acid conc. (M) & Sugar conc. $(\%)$ \\
\hline Initial concentration & & 0.11 & 0.00 \\
\hline Final concentration & & 0.76 & 0.04 \\
\hline Initial volume (ml) & & 400 & \\
\hline Final volume (ml) & & 470 & \\
\hline Delta concentration (M) & & 0.66 & \\
\hline \multicolumn{4}{|l|}{ Dilute stream: } \\
\hline & & Acid conc. (M) & Sugar conc.(\%) \\
\hline Initial concentration & & 1.03 & 0.86 \\
\hline Final concentration & & 0.01 & 1.22 \\
\hline Initial volume (ml) & & 400 & \\
\hline Final volume $(\mathrm{ml})$ & & 330 & \\
\hline $\begin{array}{l}\text { Power consumption } \\
\text { (kWh/kg of acid removal) }\end{array}$ & & 2.36 & \\
\hline Current Efficiency\% & & 69.34 & \\
\hline
\end{tabular}


Table 9. Results of electrodialysing of acid-sugar solutions at $6.0 \mathrm{~V}$ applied potential using EVOH based membranes.

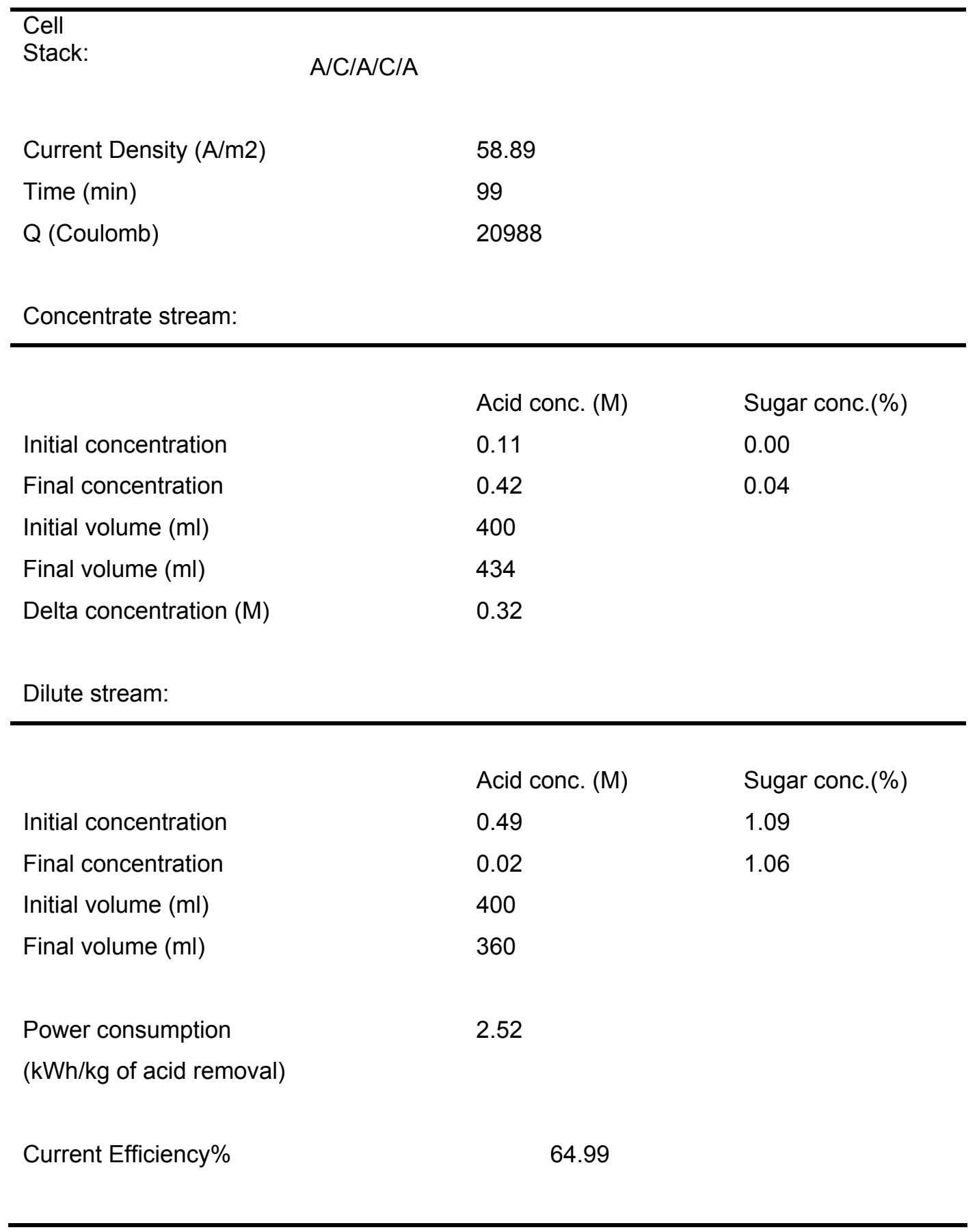


Table 10. Results of electrodialysing of acid-sugar solutions at $6.0 \mathrm{~V}$ applied potential using EVOH

Cell

Stack:

Current Density (A/m2)

Time (min)

Q (Coulomb)

Concentrate stream:

\begin{tabular}{lll}
\hline & & \\
& Acid conc. (M) & Sugar conc.(\%) \\
Initial concentration & 0.11 & 0.00 \\
Final concentration & 0.63 & 0.07 \\
Initial volume (ml) & 400 & \\
Final volume (ml) & 440 & \\
Delta concentration (M) & 0.52 & \\
& & \\
Dilute stream: & & 1.14 \\
\hline & & 1.09 \\
Initial concentration & Acid conc. (M) & \\
Final concentration & 0.79 & \\
Initial volume (ml) & 0.02 & \\
Final volume (ml) & 400 & \\
& 330 & \\
Power consumption & & \\
(kWh/kg of acid removal) & 2.88 & \\
Current Efficiency\% & & \\
& & \\
\hline
\end{tabular}

74.85

147

39610 
Table 11. Results of electrodialysing of acid-sugar solutions at $6.0 \mathrm{~V}$ applied potential using EVOH based membranes.

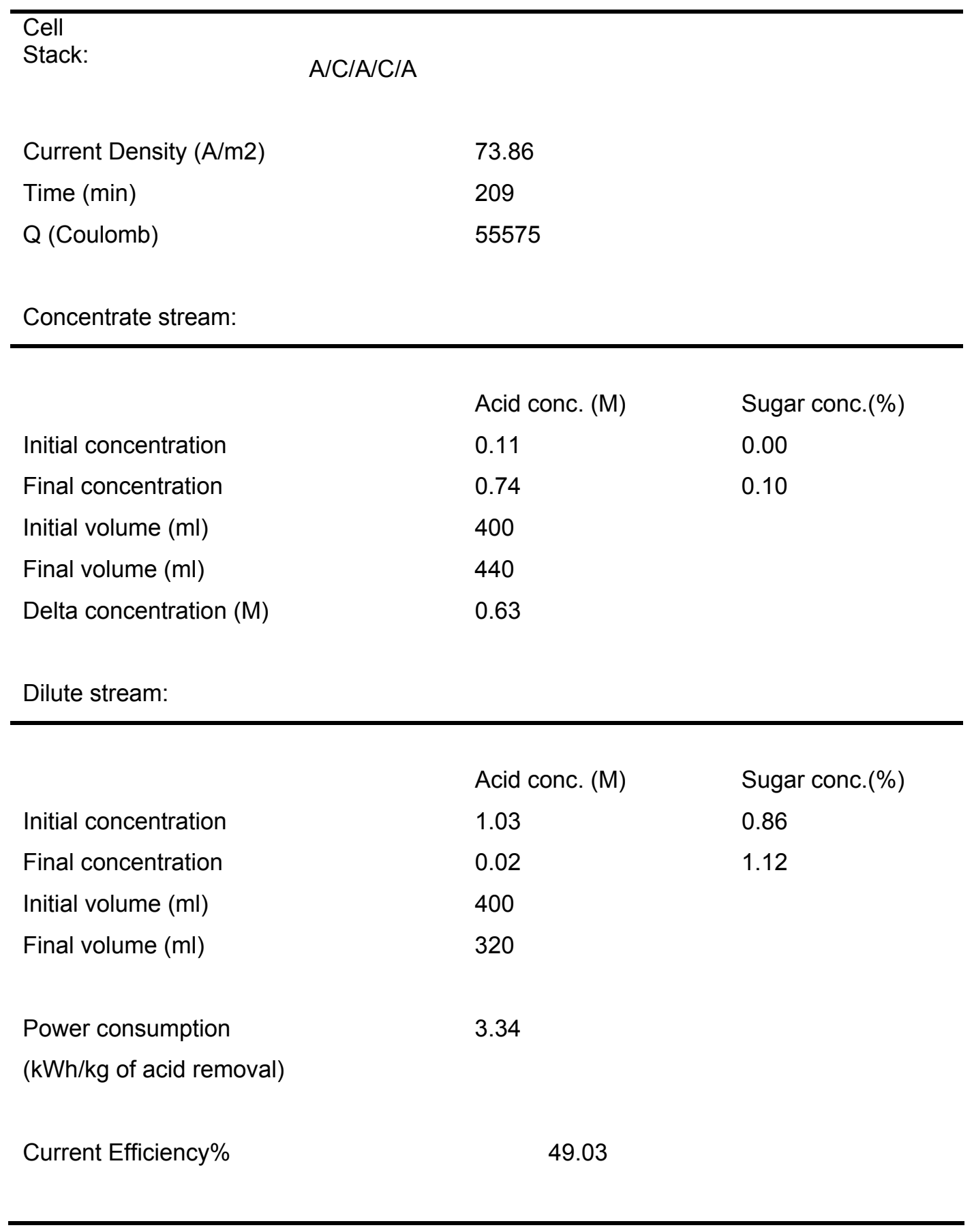


Table 12. Results of electrodialysing of acid-sugar solutions at $6.0 \mathrm{~V}$ applied potential using commercially available membranes.

\begin{tabular}{|c|c|c|c|c|c|c|}
\hline \multicolumn{2}{|c|}{ Initial acid conc. (M) } & \multicolumn{2}{|c|}{ Final acid conc. (M) } & \multirow{2}{*}{$\begin{array}{l}\text { \% Acid } \\
\text { Transferred }\end{array}$} & \multirow{2}{*}{$\begin{array}{l}\Delta \text { Final Acid } \\
\text { Conc. (M) }\end{array}$} & \multirow{2}{*}{$\begin{array}{l}\text { Current } \\
\text { Efficiency } \\
(\%)\end{array}$} \\
\hline Diluate & Concentrate & Diluate & Concentrate & & & \\
\hline 0.5 & 0.11 & 0.01 & 0.45 & 78.69 & 0.45 & 70.36 \\
\hline 0.79 & 0.11 & 0.01 & 0.66 & 83.73 & 0.65 & 73.78 \\
\hline 1.03 & 0.11 & 0.01 & 0.76 & 77.09 & 0.75 & 69.34 \\
\hline
\end{tabular}

Table 13. Results of electrodialysing of acid-sugar solutions at $6.0 \mathrm{~V}$ applied potential using EVOH based membranes.

\begin{tabular}{lllllll}
\hline \multicolumn{2}{l}{ Initial acid conc. (M) } & \multicolumn{2}{l}{ Final acid conc. (M) } & $\%$ Acid & $\Delta$ Final Acid & $\begin{array}{l}\text { Current } \\
\text { Efficiency }\end{array}$ \\
Diluate & Concentrate & Diluate & Concentrate & Transferred & Conc. (M) & $(\%)$ \\
0.49 & 0.11 & 0.02 & 0.42 & 74.34 & 0.40 & 64.99 \\
0.79 & 0.11 & 0.02 & 0.63 & 75.87 & 0.61 & 56.92 \\
1.03 & 0.11 & 0.02 & 0.74 & 69.51 & 0.72 & 49.03 \\
\hline
\end{tabular}




\section{DEVELOPMENT OF A CONCENTRATED ACID HYDROLYSIS PROCESS TO CONTINUOUSLY CONVERT SAWDUST TO SUGARS THAT CAN BE FERMENTED TO ETHANOL Performer: University of Southern Mississippi}

\section{EFFORT SUMMARY}

Commercial scale up of an acid hydrolysis process to convert waste biomass to sugars has been previously prohibited by poor economics. However, economics are now favorable with advancements in acid-sugar solution separation technologies and the development of a new concentrated-acid lignocellulosic conversion process. The new concentrated acid hydrolysis process, developed at the University of Southern Mississippi with the support of EPSCoR, uses a twin screw extruder reactor to produce near theoretical conversion of wood waste to sugars using short reaction times with minimal production of degradation products. The new process is capable of producing the sugars needed to make fuel ethanol by fermentation.

The hydrolysis process produces an acid reduced product by combining pinewood sawdust and concentrated acid within a high intensity shear environment of a co-rotating twin-screw extruder reactor. The acid-reduced product made at base case twin-screw reactor operating conditions shows a $38.2 \%$ conversion of dry sawdust solids to soluble liquids. Of this $38.2 \%$ conversion of dry solids to soluble liquids, $73.8 \%$ of all hemicellulose and $44.4 \%$ of all cellulose is converted to soluble monomers and oligiosaccharides. This high conversion was realized by an increase in reactive surface area through a reduction in average sawdust particle size due to the shear stresses present when mixing materials in the twin-screw extruder at elevated temperatures. 


\section{CELLULOSE BASED ADSORBENT MATERIALS FOR THE DEHYDRATION OF ETHANOL USING THERMAL SWING ADSORPTION \\ Performer: Mississippi State University}

\section{INTRODUCTION}

During the production of ethanol large quantities of water are produced with the ethanol. The fermenter effluent typically has an ethanol concentration of approximately 10 percent by weight. This ethanol/water mixture must be separated to produce ethanol with an ethanol concentration of $99.5 \mathrm{wt} \%$ or higher. Alcohol for automobile usage is refined through distilling processes to a maximum ethanol concentration of approximately $95.6 \mathrm{wt} \%$ and then another separation method is required. This second step is typically referred to as the dehydration step and is usually an adsorption process.

An industrial column is packed with an adsorbent that will preferentially adsorb water, leaving pure ethanol as the effluent product. Using this method of separation requires identifying adsorbents that will not only readily adsorb water during the adsorption phase of operation but also will easily desorb the water during the regeneration phase [4] [5].

Adsorbents that readily adsorb water have already been examined and are in commercial use [6] [7]. Molecular sieves were the first adsorbent to be used. The biggest drawback with molecular sieves is the high cost of regeneration because of the high temperatures required to desorb the water that collects within the pores [1] [2] [6].

This has driven the search for new and more efficient adsorbents. Dr. Michael Ladisch at Purdue University has investigated the use of natural materials as adsorbents [8]. In the early 1980's, he proposed and patented the use of corn grits as a water adsorbent [10]. With this discovery, a new approach to the ethanol dehydration problem emerged. It was found that biomass materials were generally less expensive to regenerate when compared to the molecular sieves [9]. Also, if regeneration was not feasible, the biomass adsorbent could be used as a feedstock to the fermenter at the forefront of the chemical process.

Biomass materials that have been investigated and found to be viable adsorbents include corn grits, potato starch, amylose, and maize starch [11] [12] [13] 
[3]. These materials are starch-based adsorbents with high levels of amylopectin. Another class of adsorbents, such as wheat straw and woodchips, are derived from cellulosic-based materials. These materials use xylans and hemicelluloses as the major adsorbing mechanism instead of amylopectin. Little research, however, has been performed on cellulosic materials. It is currently unclear which materials will provide the highest degree of adsorption at the lowest cost.

The cost factor is assessed, not only in terms of water uptake efficiency, but also on regeneration or adsorbent replacement costs. If the material is to be replaced after each adsorption run, then the adsorbent must be cheaply, readily available, and easily and safely disposed. TSA is more appealing than membrane separation, which uses costly vacuum and refrigeration equipment. Industrially an array of columns, some of which are in the adsorption phase and some of which are in the desorption phase, is necessary.

\section{METHODS AND MATERIALS}

A thermal swing desorption system was employed to remove the water from the adsorbent. A glass column apparatus with a $1.0 \mathrm{~L}$ flask was employed to determine the adsorption of water in gas-phase conditions. A mixture of ethanol and water was heated to produce a vapor stream that traveled upwards through the vacuum jacketed column packed with adsorbent. A column with an inside diameter of 1.0 inch (25 mm) and a length of 35 inches $(89 \mathrm{~cm}$ ) was used to provide sufficient column length and an adequate amount of adsorbent material to make dynamic column calculations possible. An inch of inactive packing made of 316 SS was placed at the bottom of the column to ensure an even radial distribution of the vapor stream.

The ethanol/ water mixtures were blended using 200-proof ethanol and distilled water. Concentrations of 90,95 , and $97 \mathrm{wt} \%$ ethanol were used to evaluate adsorption characteristics at, above, and below the azeotropic concentration of $95.6 \mathrm{wt} \%$ ethanol. This study was limited to the normal boiling point of an ethanol/water mixture near the azeotrope. Distillation of ethanol/water from a fermentation broth concentration to near the azeotrope is cheaper and more attractive than using a single adsorption step. The adsorbents used in this study were oak chips, kenaf core, and bleached wood pulp. 
The experimental runs were terminated when the water composition of the condensate equaled that of the feed. This usually took between 45 to 70 minutes, depending upon the adsorbent and the feed composition. After breakthrough of the water occurred, the adsorbent was regenerated using an air stream heated to $80^{\circ} \mathrm{C}$ and flowing at $\sim 4.5 \mathrm{cfh}$ overnight.

\section{RESULTS}

Due to the ample literature on the use of molecular sieves to dehydrate ethanol, Type A molecular sieves were used to verify proper design and use of the laboratory batch column. The column, which had an inside cross sectional area of $5.07 \mathrm{~cm} 2$ and a vapor velocity of $3.29 \mathrm{~mm} / \mathrm{s}$, was chosen to yield a volumetric flow rate of approximately $1.00 \mathrm{~cm} 3 / \mathrm{min}$. The results showed similar adsorption capacities for the molecular sieves, the oak sawdust, and the kenaf core. The mass of water adsorbed per unit mass of adsorbent increases for both the molecular sieves and the cellulosic adsorbents as the water concentration in the ethanol/ water mixture increases from 1 to $10 \mathrm{wt} \%$.

\section{Oak Sawdust}

The sawdust was sifted in a 40-mesh screen to remove fine particles and in a 10mesh screen to remove large particles. The vapor flow rate of the hardwood, less than $1.00 \mathrm{ml} / \mathrm{min}$, was the smallest of all the adsorbents, which is reflected in the time of breakthrough in the adsorption curves.

For the first few experimental runs using oak sawdust, extractives leached from the wood chips and settled into the boil-up flask, giving a brown color to the ethanol/water mixture. After several repeated runs, all of the extractives appeared to have been removed. Nevertheless, the extractives did not affect the woodchips' ability to adsorb water.

\section{Bleached Wood Pulp}

The bleached wood pulp (BWP) used in this experiment was supplied in a commercially available, flat sheet form that had to be repulped using a blender and water. After drying, the adsorbent was ready for use in the adsorption column. As can 
be seen from the breakthrough curves, the BWP preferentially adsorbed water over ethanol in ethanol/water solutions of 3, 5, and $10 \mathrm{wt} \%$ water.

An important consideration with the BWP is that it has an increasing ability to swell inside the column with increasing concentrations of water. The adsorbent swelling causes a significant pressure drop across the adsorber. This, in turn, results in column flooding and poor mass transfer of water from the fluid to the adsorbent. The swelling is due to the lack of lignin within the structure of the cellulosic material. It is the lignin that gives the adsorbent rigidity. It acts as a glue to hold cellulose and hemicellulose molecules together. Without the lignin, the adsorbent, on a per weight basis, has a higher capacity to adsorb water; however, the swelling effect is counter-productive in an adsorber column.

\section{Kenaf Core}

The kenaf core had the shortest breakthrough time of the three cellulosic materials. There are several reasons for this. First, compared to the hardwood sawdust, a higher vapor flow rate was obtained resulting in more water per unit time that came into contact with the adsorbent. Also, its bulk density is only about one-half that of the hardwood sawdust. Therefore, fewer grams of adsorbent can be placed inside the adsorption column, leaving less surface area, per unit volume, for adsorption to occur. Secondly, compared to the bleached wood pulp there are fewer polysaccharides, per unit mass, which provide the hydroxyl groups, needed to adsorb the water molecules.

\section{COMPARISON OF ADSORBENTS}

For all three adsorbents that were studied, as the concentration of water increases, shorter breakthrough times occur. This is because more water has been added to the system, and therefore, the adsorbent reaches its capacity quicker. The experiments conducted at the $95 \mathrm{wt} \%$ ethanol level prove that the ethanol can be purified past its azeotropic point. The experiments conducted at the $90 \mathrm{wt} \%$ ethanol level demonstrate the possibility of operating an industrial adsorption column in which the feed is somewhat below the azeotropic point. 
The bleached wood pulp demonstrated the highest water loading followed by the kenaf core and then the oak sawdust. Breakthrough curves at the $90 \mathrm{wt} \%$ ethanol level were not obtained due to a lack of column length, and therefore, insufficient adsorbing material to remove enough water to reach the breakthrough concentration of $99.5 \mathrm{wt} \%$ ethanol.

\section{DISCUSSION OF RESULTS}

Adsorption is a surface phenomenon; therefore, the binding of water to an adsorbent is occurring at the surface. The major constituents of cellulosic-based adsorbents are cellulose, hemicellulose, and lignin. The cellulose is composed of a moderately crystalline matrix with long microfibrils that are wound around a fiber axis. The microfibrils are joined together through hydrogen bonding and are embedded within an amorphous medium of hemicellulose. The lignin acts as a glue to hold together the other components and stiffens the cell walls.

When designing an adsorber to remove water from a mixture of ethanol and water, it is desired to find an adsorbent that is amorphous rather than crystalline. Crystalline structures have a highly ordered molecular arrangement and leave little room for additional molecules to be added. Cellulose has a high degree of crystallinity, up to $80 \%$, whereas, hemicellulose is essentially amorphous. The lignin, on the other hand, adds very little to the water adsorption quality.

The interactions between molecules of water and the hydroxyl groups attached to the polysaccharides are rather involved. As the $\mathrm{H}_{2} \mathrm{O}$ molecule is brought into contact with the $\mathrm{OH}^{-}$group, one of the hydrogen's of the $\mathrm{H}_{2} \mathrm{O}$ molecule becomes the proton donor, and the other hydrogen becomes the proton acceptor. Only the hydroxyls located on the adsorbent surface are available to water adsorption. That is, hydroxls that are wound within the inner microfibril matrix are not accessible to water molecules. The adsorbed molecules of water perturb the surface $\mathrm{OH}^{-}$groups creating shifts of their vibrations to lower frequencies. The free $\mathrm{OH}^{-}$groups have a shorter wavelength than the disrupted ones.

Therefore, a material such as bleached wood pulp is theoretically ideal because the lignin has been removed and it is an amorphous material with a high concentration of xylans. Experimentally, however, it does have its drawbacks. Without the lignin, 
there is no adhesion between cell walls to give the material a rigid structure. Consequently, the fibers swell with the adsorption of water providing a significant pressure drop inside the column between the boil-up flask and the condenser.

The kenaf core is considered a non-wood fiber and has a lignin content of $18 \%$. As the lignin content increases, the cellulose and hemicellulose contents decrease. As a result, fewer hydroxyl groups are available and fewer water molecules are adsorbed. It does have a higher concentration of xylans, on a per weight basis, than the hardwood sawdust.

\section{Mass Transfer Zone Properties}

From the results of the breakthrough curves, the dynamic characteristics of the Mass Transfer Zone (MTZ) can be calculated. Table 2 summarizes the values of the parameters of the MTZ as well as the values of the length and velocity of the MTZ. The MTZ is an integral part in designing adsorption columns. Only experiments conducted at the 95 and $97 \mathrm{wt} \%$ ethanol were used to calculate MTZ properties. Experimental results from the $90 \mathrm{wt} \%$ ethanol lacked sufficient column length, and therefore, adsorbent volume to produce the necessary data for calculation of MTZ properties.

\section{CONCLUSION}

Ligno-cellulosic materials possess an affinity towards water. Their hygroscopic nature allows for the uptake of water molecules from ethanol mixtures that are at least 10 percent, by weight, water. All three materials studied demonstrated the ability to preferentially adsorb water over ethanol. These materials all contain an abundance of hydroxyl groups from their molecular makeup that are necessary to adhere water molecules to the surface of the adsorbent.

At the threshold point, called breakthrough, the bleached wood pulp performed best, followed by the kenaf core and then the hardwood sawdust. This can only be stated for concentrations of 3 and 5 wt $\%$ water. There was insufficient data at the 10 wt $\%$ water due to equipment capability. The data did show adsorption of water at this concentration; however, it was an insufficient amount to produce ethanol with at least a 99.5 wt\% purity. It is believed that an increase in column length, resulting in more 
adsorbent, would give results similar to those obtained with the 95 and $97 \mathrm{wt} \%$ concentrations.

The length of the Mass Transfer Zone (MTZ) and the velocity at which it travels through the adsorbent bed increases with increasing concentrations of water. This is a rather intuitive observation. As the concentration of water increases, the adsorbent will reach its threshold at a much faster rate. The bleached wood pulp has the shortest MTZ lengths and velocities, followed by the kenaf core and then the hardwood sawdust. All breakthrough curves are wide indicating long MTZ lengths. Longer adsorbent beds will make more efficient use of the bed capacity and lower regeneration costs.

Bleached wood pulp is a superior adsorbent over the kenaf core and hardwood sawdust in adsorbing water due to its large percentage of hydroxyl groups stemming from a high concentration of xylans. The bleached wood pulp was the only material that demonstrated similar water adsorbing capacities as compared to starch-based adsorbents such as corn grits. 


\section{REFERENCES}

1. Al-Rub, Fahmi rt al., (1999), Separation Science and Technology, 34, $2355-2368$ (1999).

2. Banat, Fawzi et al., Separation and Purification Technology, 18, 111 - 118 (2000).

3. Bienkowski, P. et al., Biotechnology and Bioengineering, 28, 960 - 964 (1986).

4. Crittenden, Barry and Thomas, W. John, "Adsorption Technology and Design" (1998).

5. Crittenden, B. D. and Sowerby, B Inst of Chem Eng Symp Series, 118 (1990).

6. Davis, Karl G. and Manchanda, Kristen Chemical Engineering (1974).

7. Grethlein, Hans E. and Nelson, Thomas B., USDA, Agricultural Research Service 1992).

8. Ladisch, Michael R., Science, 205, 898 - 900 (1979).

9. Ladisch, Michael R., et.al. Ind. \& Eng. Chem. Proc Design and Dev., 23, 437 - 443 (1984).

10. Ladisch, Michael R., and Tsao, G. T., U. S. Patent No. 4,345,973 (1982).

11. Lee, Jay Y., Ladisch, Michael R., Biochem Eng 5492 - 498 (1987).

12. Lee, Jay Y. et al., AICHE Journal, 37, 1187 - 1195 (1991).

13. Neuman, Robert, et al. American Chemical Society (1986). 


\section{FIGURE}

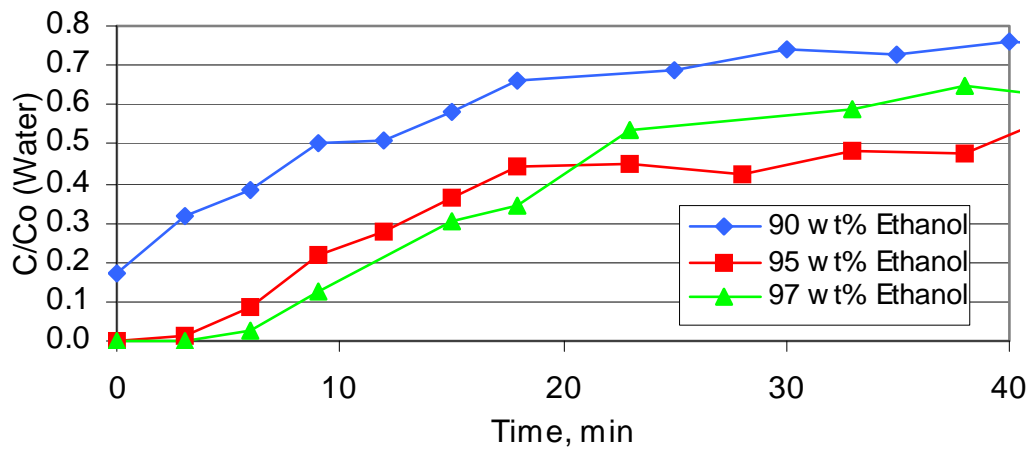

(a)

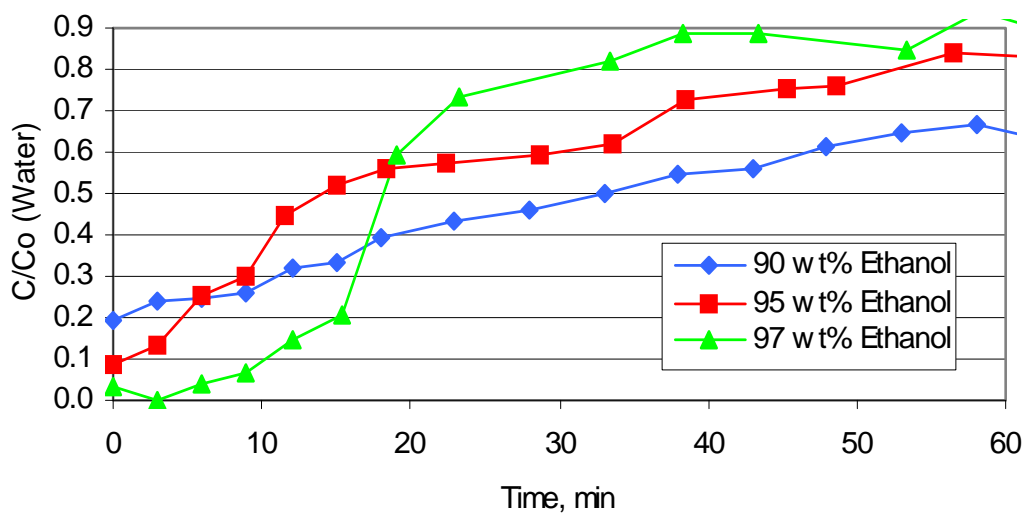

(b)

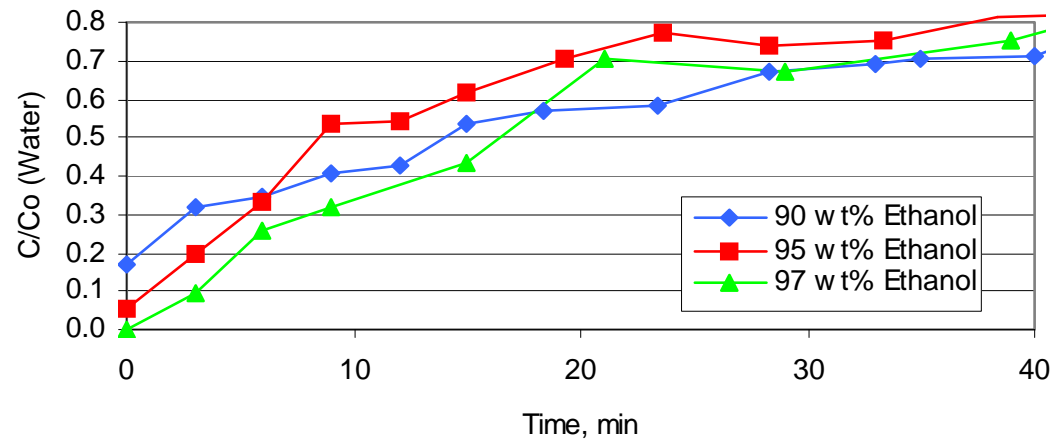

(c)

Figure 1. Breakthrough curves demonstrating adsorption characteristics (a) bleached wood Pulp, (b) oak chips, and (c) kenaf core 


\section{TABLES}

Table 1. Conditions and results for vapor phase column experiments

\begin{tabular}{|l|c|c|c|c|c|c|}
\hline Adsorbent & $\begin{array}{c}\text { Inlet } \\
\text { Composition } \\
\text { Ethass Fraction } \\
\text { Ethanol }\end{array}$ & $\begin{array}{c}\text { Vapor Flow } \\
\text { Rate } \\
(\mathrm{ml} / \mathrm{min})\end{array}$ & $\begin{array}{c}\text { Breakthrough } \\
\text { Time } \\
(\mathrm{min})\end{array}$ & $\begin{array}{c}\text { Water } \\
\text { Adsorbed } \\
(\mathrm{g})\end{array}$ & $\begin{array}{c}\text { Water } \\
\text { Loading }\end{array}$ & $\begin{array}{c}\text { Water } \\
\text { Adsorption } \\
\text { Rate }^{3}\end{array}$ \\
\hline Oak Chips & 0.97 & 0.655 & 14.5 & 0.285 & 0.0052 & 3.786 \\
\hline Oak Chips & 0.95 & 0.795 & 3 & 0.119 & 0.0022 & 0.7660 \\
\hline Oak Chips & 0.90 & 0.766 & - & - & - & - \\
\hline Kenaf Core & 0.97 & 0.953 & 7 & 0.200 & 0.0089 & 1.2758 \\
\hline Kenaf Core & 0.95 & 0.942 & 3 & 0.141 & 0.0063 & 2.1017 \\
\hline Kenaf Core & 0.90 & 1.010 & - & - & - & - \\
\hline BWP & 0.97 & 0.989 & 13 & 0.3857 & 0.0125 & 0.9622 \\
\hline BWP & 0.95 & 1.019 & 7 & 0.3567 & 0.0116 & 1.6524 \\
\hline BWP & 0.90 & 0.855 & - & - & - & - \\
\hline
\end{tabular}

Notes:

${ }^{1}$ Breakthrough occurs at an exiting concentration of 99.5 wt\% Ethanol.

${ }^{2}$ Water Loading is given by grams $\mathrm{H}_{2} \mathrm{O}$ adsorbed per gram adsorbent ( $\mathrm{g} \mathrm{H}_{2} \mathrm{O} / \mathrm{g}$ ads).

${ }^{3}$ Water Adsorption Rate is given by grams $\mathrm{H}_{2} \mathrm{O}$ adsorbed per kilogram adsorbent per minute $\left(\mathrm{g} \mathrm{H}_{2} \mathrm{O} / \mathrm{Kg}\right.$ ads/min). 
Table 2. Parameters and characteristics of the Mass Transfer Zone

\begin{tabular}{|l|c|c|c|c|c|c|}
\hline Adsorbent & $\begin{array}{c}\text { Inlet } \\
\text { Composition } \\
\text { (wt frac etoh) }\end{array}$ & $\begin{array}{c}\text { Length } \\
\text { of Bed } \\
(\mathrm{cm})\end{array}$ & $\begin{array}{c}\text { Time } \mathrm{C}_{\mathrm{o}} / 2 \\
\text { Occurrence } \\
(\mathrm{min})\end{array}$ & $\begin{array}{c}\Delta \mathrm{t} \\
(\mathrm{min})\end{array}$ & $\begin{array}{c}\text { Velocity } \\
\text { of MTZ } \\
(\mathrm{cm} / \mathrm{min})\end{array}$ & $\begin{array}{c}\text { MTZ } \\
\text { Length } \\
(\mathrm{cm})\end{array}$ \\
\hline Oak Chips & 0.97 & 89 & 18.0 & 23.0 & 3.71 & 85.3 \\
\hline Oak Chips & 0.95 & 89 & 14.0 & 47.5 & 6.36 & 302.0 \\
\hline Kenaf Core & 0.97 & 89 & 19.0 & 36.5 & 4.68 & 171.0 \\
\hline Kenaf Core & 0.95 & 89 & 12.0 & 33.0 & 7.42 & 244 \\
\hline BWP & 0.97 & 89 & 28.5 & 24.0 & 3.12 & 74.9 \\
\hline BWP & 0.95 & 89 & 37.5 & 41.0 & 2.37 & 97.3 \\
\hline
\end{tabular}

Note: ${ }^{1}$ Time required for the water concentration in the column effluent to reach one-half of its initial concentration. (i.e. for oak chips, the time required for composition of the column effluent to reach 0.985 wt. fraction ethanol with an inlet composition of 0.97 wt. fraction ethanol was 18 minutes.) 


\section{DEVELOPMENT OF A PERSTRACTION MEMBRANE SEPARATION METHOD Performer: Mississippi State University}

\section{SUMMARY}

The study of ethanol recovery technologies will include the study of separating ethanol from the fermenters liquid effluent by membranes. A study was made using perstraction with a polycarbonate membrane. Perstraction solvents studied were water, hexane, tri-butyl phosphate, and 2 ethyl-1-butanol.

It was found that water has the greatest chance of success as an extraction solvent. Water not only worked well as a perstraction unit, it is not toxic if crosscontamination of solvent occurs, which was the case with the other solvents tested.

The objective is to remove hexane from the fermenter in-situ in order to reduce the effect of ethanol inhibition. This is accomplished through a process called perstraction, where an intermediate solvent is passed on one side of a membrane with fermenter broth on the opposite side. The ethanol passes through the semi-permeable membrane from the fermenter side to the solvent side. Since the ethanol is removed on a continuous basis, the ethanol concentration in the fermenter is held to a low enough level that toxic effects on the ethanol producing bacteria are minimized. The ethanol taken up by the solvent can then be recovered by conventional concentration and/or distillation.

In this study, a hydrophobic, polypropylene, hollow fiber membrane module model G543 manufactured by Liqui-Cel was used to evaluate the effectiveness of the perstraction of ethanol from a mixture of ethanol and water. The fiber membrane had an outer diameter of $300 \mu \mathrm{m}$ and an inner diameter of $200 \mu \mathrm{m}$. Solutions of ethanol in water at concentrations of $10,7.5$, and 5 weight percent ethanol were used as the simulated fermentation broth. 1-octanol, tri- $n$-butylphosphate, hexane, 2-ethyl-1butanol, and water were used as the solvents. Water proved to be the most effective and the least toxic solvent. Fick's Law diffusion coefficients were determined for ethanol diffusing from the shell side to the lumen side of the membrane.

Experimental results indicate that with a water solvent, as the flow rate of solvent on the lumen side increases, the diffusion coefficient decreases. Also, the dependence of temperature on diffusion coefficient of the ethanol-water solvent system was studied 
at 22, 30, and 35 degrees Celsius. The diffusion coefficient varied from a minimum value of $3.58 \mathrm{E}-11 \mathrm{~m}^{2} / \mathrm{s}$ at $22 \mathrm{C}^{\circ}$ to a maximum of $1.38 \mathrm{E}-09 \mathrm{~m}^{2} / \mathrm{s}$ at $35 \mathrm{C}^{\circ}$ over the range of concentrations and flow rates tested.

1-octanol, tri- $n$-butylphosphate, hexane, and 2-ethyl-1-butanol all functioned as solvents, but the solvent was found to have leaked into the fermenter broth side of the membrane, thus posing as a contaminant to the fermentation process. Therefore, they are not usable as solvents in this process. The tri-n-butylphosphate also proved not to be compatible with the polycarbonate membrane and tygon tubing used in the experimental apparatus.

The test method developed will allow different membranes to be evaluated and their diffusivities determined. With knowledge of diffusivities, sizing calculations may be made to design larger scale equipment. However, before this technology can go to commercial scale, pilot scale operations should be conducted.

\section{Membrane Module}

The membrane module used was a model G543 minimodule manufactured by Liqui-Cel Corporation, Charlotte, NC. It is $30 \mathrm{~mm}$ in diameter and $156 \mathrm{~mm}$ in length. The materials of construction are polycarbonate and polyurethane. It is illustrated in Figure 1.

\section{Experimental Apparatus}

The experimental apparatus (Figure 2.) consisted of two agitated storage flasks, one containing simulated fermenter liquid and one containing the perstraction solvent. Each vessel was connected to a variable volume peristaltic pump that had the capability of pumping between 0 and $500 \mathrm{ml}$ of liquid per minute. The pumps in each case were connected to one of the inlets of the membrane device. The outlets were routed back to the original vessel from which the fluid was being pumped; hence, each fluid was being recirculated. The ethanol concentrations were in some cases determined by gas chromatography and in other cases concentrations were determined by refractive index. Back pressure was controlled on the outlet of the fermenter unit by means of manually regulating a discharge valve to maintain a preset pressure by monitoring a pressure gauge. 


\section{RESULTS}

Various runs were made using a simulated fermenter solution of ethanol and water. Various solvents were used, with water being the most practical solvent used. Data from a typical perstraction run is shown in Figure 4. Note that as the fermenter ethanol concentration decreases, the solvent ethanol concentration increases. Ethanol transfer rate per unit area of exposed membrane was calculated. With the ethanol flux per unit area determined and with knowledge of the concentration difference over the membrane, a Fick's Law diffusion coefficient could be determined as illustrated in Figure 5. It was found that diffusion coefficient varied with ethanol concentration. Figure 6 illustrates how diffusion coefficient changes over a range of concentrations. 


\section{REFERENCES}

1. Liu, Fangfang; Liu, Li and Feng, Xanshe, "Separation of acetone-butanol-ethanol from dilute aqueous solutions by pervaporation," Science Direct-Separation and Purification Technology, Vol 42 Issue 3 pp 273-282 (April 2005)

2. Matsumura, Masatoshi and Makl, Herbert, "Elimination of ethanol inhibition by perstraction," Biotechnology and Bioengineering, Vol. XXVIII pp 534-541 (1986)

3. Udriot, H.; Ampuero, S.; Marison, I.W., and von Stockar, U. "Extractive fermentation of ethanol using membrane distillation," Biotechnology Letters Vol 11 No 7 pp 509-514 (1989) 


\section{FIGURES}

\section{Contactor Characteristics}

$1 \times 5.5$

Cartridge Configuration Parallel Flow. Lumenside Liquid Flow.

Liquid Side Flow

Guidelines $<500 \mathrm{ml} / \mathrm{min}(0.13 \mathrm{gpm})$

Membrane X50 Fiber

Porosity $40 \%$ Porosity

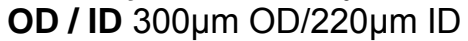

Potting Material Polyurethane

Active Surface Area $0.18 \mathrm{~m} 2(1.9 \mathrm{ft} 2)$

Number of Fibers 2300

Maximum

Temperature/Pressure

$4.2 \mathrm{~kg} / \mathrm{cm} 2(4.1 \mathrm{bar}, 60 \mathrm{psig})$ at $23^{\circ} \mathrm{C}\left(73^{\circ} \mathrm{F}\right)$

Maximum $35^{\circ} \mathrm{C}\left(95^{\circ} \mathrm{F}\right)$ at $2.1 \mathrm{~kg} / \mathrm{cm} 2(2.1 \mathrm{bar}, 30 \mathrm{psig})$

Housing Characteristics

Material Polycarbonate Housing and end caps

Flange Connections

Shellside

(Gas / Vacuum)

Standard Female Luer Lock

with Polycarbonate adaptors to $1 / 4$ inch Hosebarb

Lumenside

(Wetted Surface)

$1 / 4$ inch FNPT or

Dialyzer Type Male Luer Lock with ABS adaptors to $5.9 \mathrm{~mm}(0.23$

inch) Straight tube and acetal adapters to $6.35 \mathrm{~mm}$ (0.25 inch)

John Guest ${ }^{\circledR}$ fittings

Weight (with adapters)

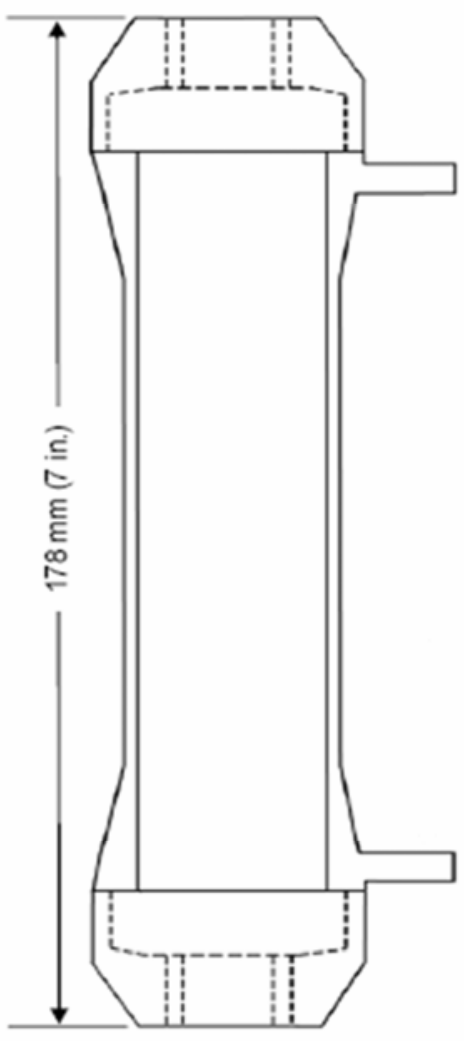

Dry $0.051 \mathrm{~kg}(0.11 \mathrm{lbs}$.)

Liquid Full (Lumenside) $0.068 \mathrm{~kg}(0.15 \mathrm{lbs}$ )

Shipping Weight $0.218 \mathrm{~kg}(0.48 \mathrm{lbs}$.)

Figure 1. Liqui-Cel Corporation Model G543 Minimodule Membrane Module 


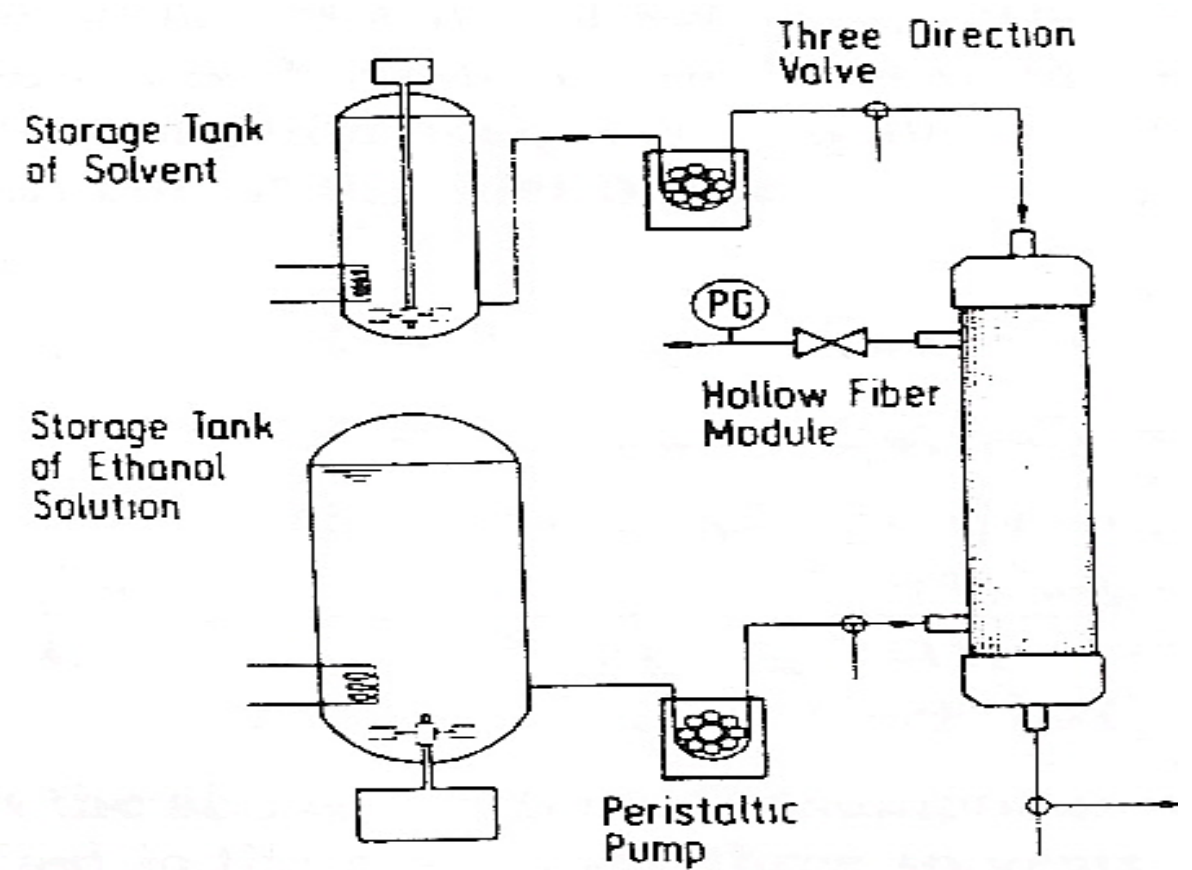

Figure 2. Schematic of experimental apparatus

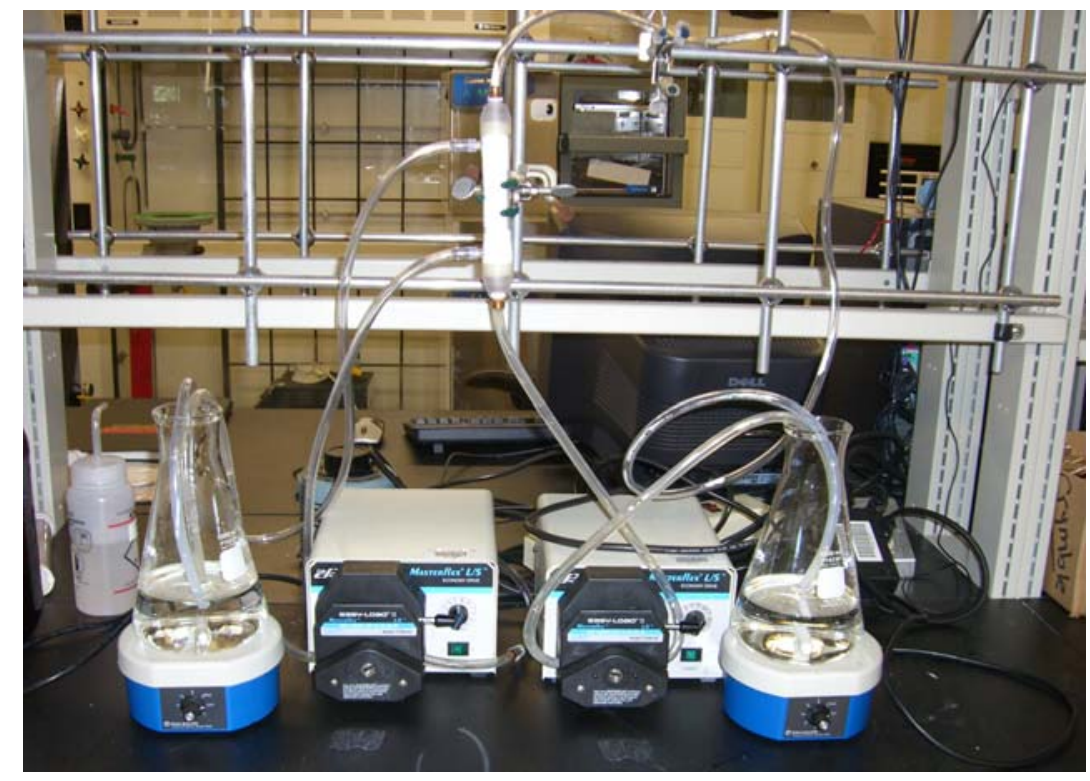

Figure 3. Photograph of experimental apparatus 


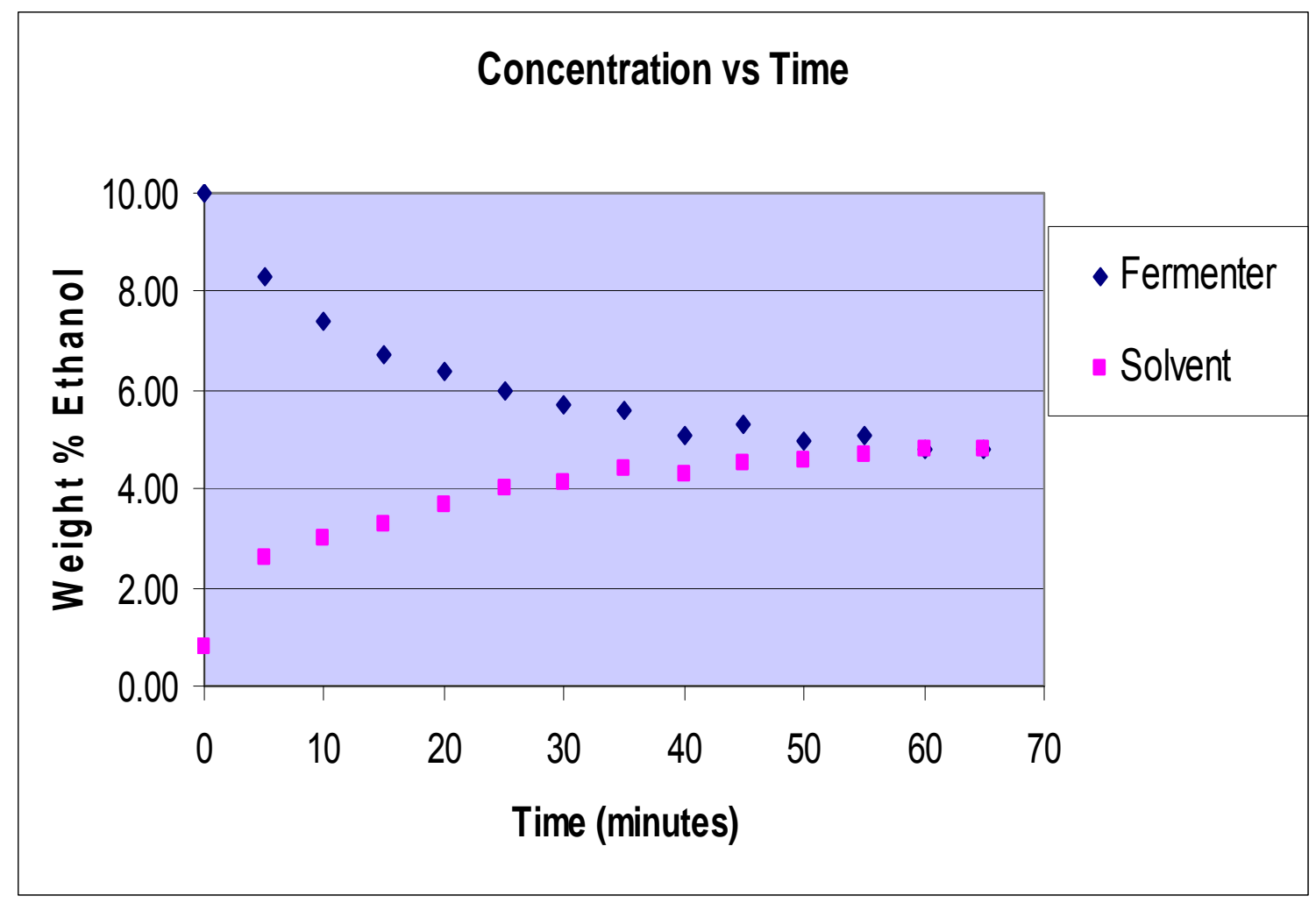

Figure 4. Perstraction run results

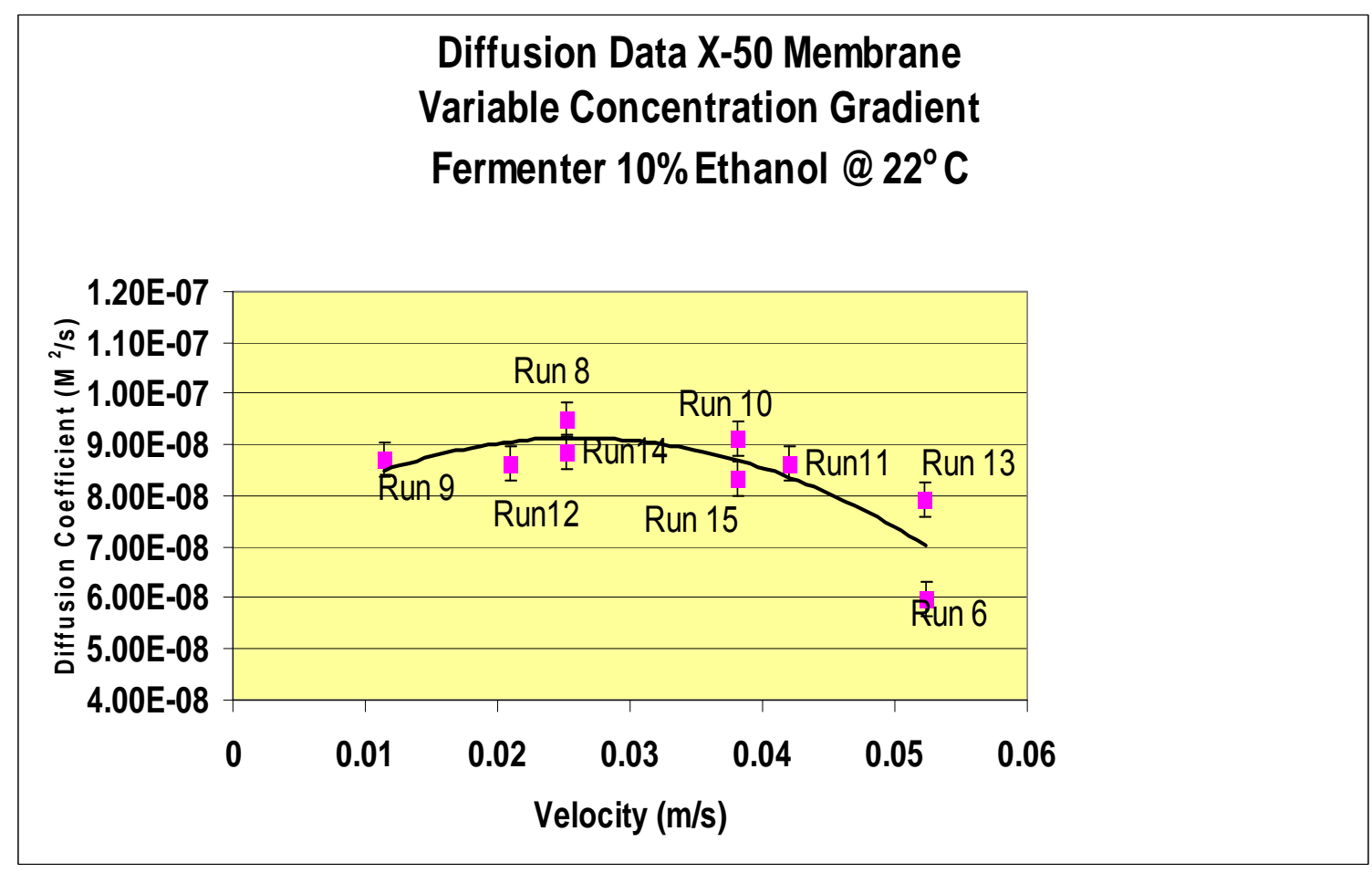

Figure 5. Diffusion coefficient for different flow rates of through membrane fibers. 


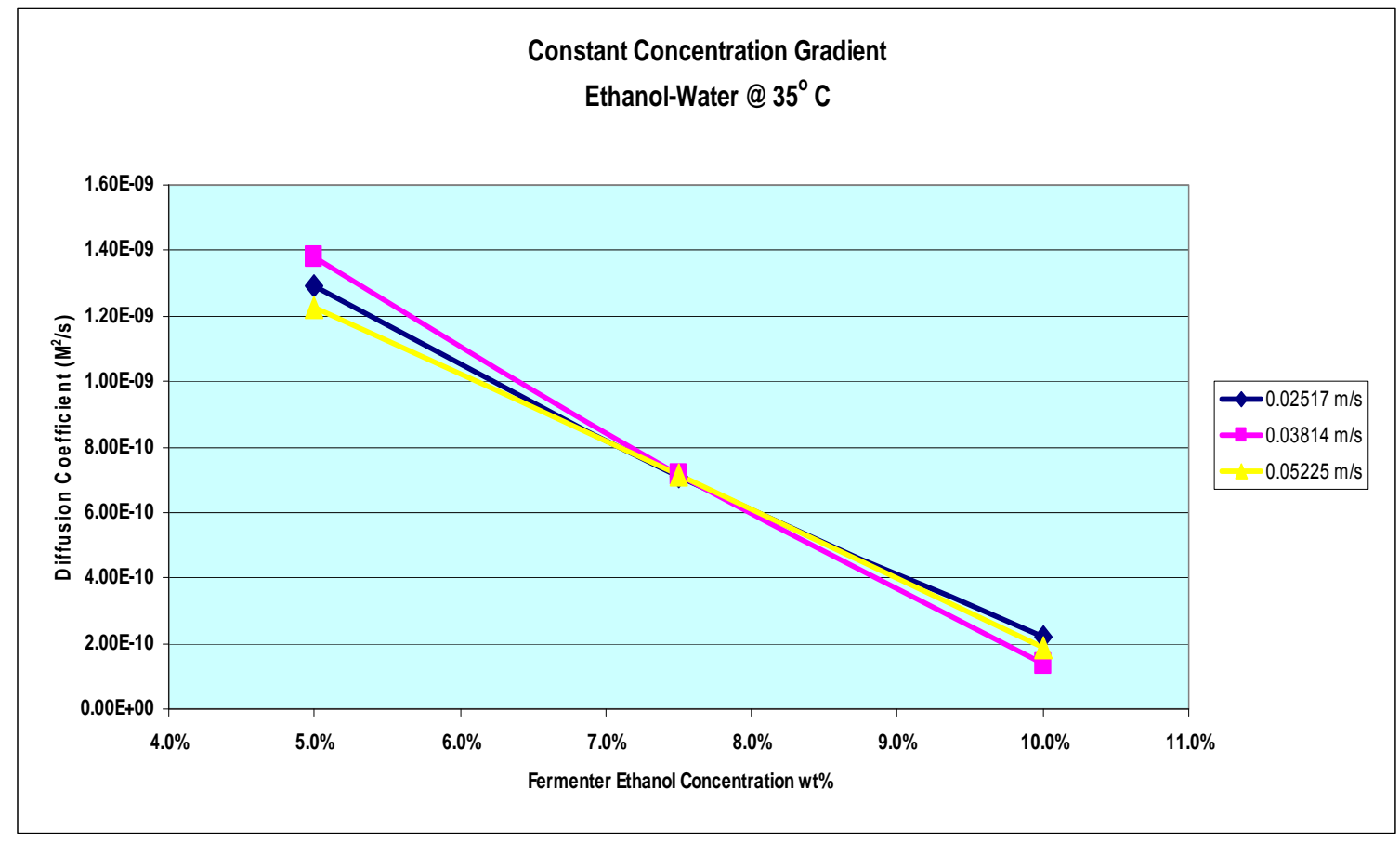

Figure 6. Variation of diffusion coefficient with fermenter concentration. 


\section{PRODUCTION OF BIODIESEL FROM WASTEWATER TREATMENT SLUDGES Performer: Mississippi State University}

\section{INTRODUCTION}

Biodiesel is a renewable displacement fuel to petroleum-derived diesel. The fuel is comprised of different fatty acid alkyl esters. These fatty acid alkyl esters are commonly produced through a transesterification reaction between methanol and triglycerides with plant oils being the most universally used. In the United States, biodiesel is predominately produced from soy oil. In 2006, Haas et al. estimated that the production cost of soy biodiesel was $\$ 2.00 /$ gal when soy oil was selling for $\$ 1.76 /$ gal. The production cost reported by these researchers also applies a $\$ 0.12 /$ gal credit for the sell of glycerol. Biodiesel producers in the US are currently stockpiling their glycerol by-product since the glycerol market has dropped due to a flooding of the market with glycerol from increased biodiesel production. If glycerol could not be sold, a more accurate biodiesel production cost would be in the neighborhood of $\$ 2.12 /$ gal. In either case, the cost of the feedstock, soy oil, represents $83-88 \%$ of the total production cost.

The cost of biodiesel production can be significantly reduced through the utilization of cheap and abundant feedstocks. Traditionally, microorganisms are thought of as the catalyst for the conversion of biomass into higher value products. A biomass source that has been largely overlooked as feedstock in the production of biodiesel is microorganisms. This new approach treats the microorganisms as the feedstock for the production of biodiesel. The lipid content of microorganisms ranges between $1-25 \%$ on a dry weight basis. Although microorganisms are ubiquitous in the environment, a concentrated source would be needed to feasibly produce biodiesel from this unique source.

Mississippi State University has identified a source of microorganisms which is generated in the US at a rate of more than a million tons a day during the treatment of sludge. This source of microorganisms is, in most cases, a burden to dispose of. Most sludge treatment facilities utilize aerobic microorganism to remove the dissolved organics in the influent and during this process carbon dioxide and cell mass are generated. In order for sewage treatment to be effective a certain amount of the cell 
mass generated must be wasted to prevent the over population of the microorganisms and subsequently loss in organic removal.

Work by Conrad et al. in 2003 has shown that sewage sludges contain an assortment of lipids such as glycolipids, phospholipids, neutral lipids, lipopolysaccarides, and free fatty acids. Since these are saponifiable lipids, biodiesel can be manufactured from them. Because the lipids in sewage sludges are mostly glycolipids and phospholipids, different extraction techniques must be identified. The objective of this study was to optimize the extraction of lipids from sewage sludge for the production of biodiesel.

\section{METHODS}

Methanol, acetone, normal butanol, toluene, 1,3-dichlorobenzene, sulfuric acid, sodium chloride, and hexane were purchased from Fisher Scientific, Atlanta, GA. These chemicals were used as received. Secondary sewage sludge was collected from a municipal wastewater treatment plant (MWWTP) located in Tuscaloosa, AL. Upon sample collection at the facility, the sludge flocks were allowed to gravity settle. Removal of free water resulted in a sludge containing $2 \%$ solids. This was dewatered by centrifugation or pressure filtration. Centrifugation was performed with $A$ Marathon 3000 centrifuge manufactured by Fisher Scientific, and operated at $3000 \mathrm{rpm}$ for 20 minutes. Removal of free water resulted in a sludge containing $7-8 \%$ solids. Pressure filtration was conducted with a Millipore $1.5 \mathrm{~L}$ pressure filter pressurized in $10 \mathrm{psi}$ increments from 15-75psi. Sludge was first filtered using an $80 \mu \mathrm{m}$ nylon filter with the filter cake collected for later use. The filtrate was then run again with a $20 \mu \mathrm{m}$ nylon filter and the cake combined with that from the $80 \mu \mathrm{m}$ run. Removal of free water resulted in a sludge containing $12-14 \%$ solids. Sludge from centrifugation and filtration processes was further treated or dried prior to organic solvent or supercritical $\mathrm{CO}_{2}$ extraction.

Prior to solvent extraction sludge was mixed with hydromatrix (manufactured by Varian, Inc., Palo Alto, CA), to absorb free water in the sample, and loaded into a steel sample vessel. Hydromatrix was added until the sample formed small pellets and flowed freely. Solvent extraction was conducted using a 200 Series Accelerated Solvent Extraction system (ASE) manufactured by Dionex, which included a multisolvent control system. The system was operated at 1500 psig and $100{ }^{\circ} \mathrm{C}$ for 1 hour 
per extraction. Single or sequential (2 or 3 times) extractions were examined using the following solvent mixtures (\% by volume) or pure solvents:

1. $60 \%$ hexane $/ 20 \%$ methanol $/ 20 \%$ acetone (same mixture 3 times)

2. Pure methanol followed by pure hexane

3. Pure methanol followed by pure hexane followed by pure normal butanol

4. Pure hexane (single extraction)

After extraction the sample vessel was drained into a glass collection vial followed by a solvent flush equal to $50 \%$ of the sample vessel's volume. The lipid-containing solvent vial was then stored at $-15^{\circ} \mathrm{C}$ until further analysis.

A fluid bed drier (Endecotts Model FBD 2000; London England) and an oven (Fisher Scientific, Pittsburgh, PA) were utilized to reduce the moisture content of the sludge to approximately $5 \%$ moisture prior to extraction using supercritical carbon dioxide. The moisture was determined using infrared heating (Ohaus Model MB45; Pine Brook, NJ). The $\mathrm{SC}-\mathrm{CO}_{2}$ extractions were performed with a lab-scale extractor manufactured by Thar Technologies Model SFE 100; Pittsburgh, PA. The extractions were conducted at $45^{\circ} \mathrm{C}$ and 4714 psi. Four sequential extractions were performed on each sludge sample. In the case where methanol was used as a co-solvent, the flow rate was based on total weight of the solvents, $3.75 \mathrm{~g} / \mathrm{min}$ and $0.50 \mathrm{~g} / \mathrm{min}$. The oil collection vessel was rinsed with $20 \mathrm{~mL}$ of hexane when using purely $\mathrm{SC}-\mathrm{CO}_{2}$ as the extraction medium. Methanol was used in addition to hexane to rinse the vessel after the co-solvent experiments to dissolve any polar lipids.

\section{Sample Analysis}

After extraction the lipid-containing solvent phase was removed under vacuum in a Büchi R205 Rotary Evaporator (rotovap) at $40^{\circ} \mathrm{C}$ and $150-300 \mathrm{mbar}$ of pressure. The resulting residue was taken to be lipids and final weight was determined using an Ohaus analytical balance. The yield of lipid was then determined through gravimetric means to give a yield of gram extractable lipid per gram of dry solid.

Conversion of lipids to FAMEs were carried out through acid catalysis using a modified version of Christie's method (Christie, 1993). $20 \mathrm{mg}$ of lipids were dissolved in $1 \mathrm{ml}$ of hexane containing 1,3-dichlorobenzene as an internal standard and added to a vial with $2 \mathrm{ml}$ of $1 \%$ sulfuric acid in methanol. The vial was then capped and heated overnight at $50^{\circ} \mathrm{C}$. Then, a $5 \mathrm{ml}$ aliquot of $5 \% \mathrm{NaCl}$ in water was added and the FAMEs 
were extracted with hexane $(2 \times 5 \mathrm{ml})$, vortexing the vial between extractions. The hexane phase was washed with $2 \%$ sodium bicarbonate and dried over sodium sulfate.

The FAMEs produced by transesterification were analyzed on an Agilent gas chromatograph (Model 6890; Palo Alto, CA) with a flame-ionization detector. The separation was achieved with a fused silica capillary column composed of stabilized 90\% polybiscyanopropyl/10\% cyanopropylphenyl siloxane (SP-2380; Supelco, Bellefonte, PA). The dimensions of the column were $100 \mathrm{~m} \times 0.25 \mathrm{~mm} \times 0.2 \mu \mathrm{m}$. A calibration curve was prepared by injecting known concentrations of an external standard mixture comprised of 37 fatty acid methyl esters (Supelco, Bellefonte, PA). 1,3-dichlorobenzene was used as an internal standard. The method consisted of injecting $1 \mu \mathrm{l}$ of sample into the GC with a split ratio of 100:1. The temperature program began at $110{ }^{\circ} \mathrm{C}$ and ended at $240{ }^{\circ} \mathrm{C}$ over a nonlinear temperature gradient of 99 minutes. Concentration data obtained from GC runs was used to calculate the amount of saponifiable material in extracted lipids.

\section{RESULTS}

\section{Efficacy of Different Solvents to Extract Oil from Sewage Sludge}

Table 1 shows the percent oil extracted using different solvent extractions on sewage sludge from the Starkville, MS sewage treatment facility. The solvents tested were Hexane $(H)$, Toluene $(T)$, Methanol $(M)$, Acetone $(A)$, Chloroform (C), n-butanol $(\mathrm{nB})$, or super critical $\mathrm{CO}_{2}\left(\mathrm{SC}-\mathrm{CO}_{2}\right)$ either separately, mixtures with one of the other solvents, or sequential with one or more other solvents. For example, a notation of $60 \mathrm{H} / 20 \mathrm{M} / 20 \mathrm{~A}$ would indicate an extraction with a mixture of $60 \%$ hexane, $20 \%$

methanol, and $20 \%$ acetone. In the same way, $100 \mathrm{M} / 100 \mathrm{H}$ is a sequential extraction of $100 \%$ methanol followed by $100 \%$ hexane.

The results show little difference in oil yield when non-polar solvents are the only solvent utilized or when the non-polar solvent is used first. However when methanol is the solvent used initially the oil yield is increased dramatically. $\mathrm{SC}-\mathrm{CO}_{2}$ extracted as well as any of the solvents or solvent combinations tested with the exception of methanol with yields of 13.02 and $9.23 \%$ in separate extractions. The solvent systems that utilized a polar solvent initially clearly had higher oil yields. 


\section{Effects of Sonication on Lipid Extraction}

Since the majority of the lipids associated with sewage sludge would be bound lipids, sonication was tested to determine its effectiveness to free bound lipids. The results of these experiments are given in Table 2. In the first set of experiments, sewage sludge from Starkville, MS was concentrated via centrifugation to $\sim 8 \%$ solids by weight. The sludge was then sonicated at an amplitude of $50 \%$ for 1 hour in an ice bath. The sonicated-sludge was mixed with hydromatrix, divided, and extracted on the ASE at 1500 psig and $100^{\circ} \mathrm{C}$ for 1 hour using either $100 \%$ hexane as the solvent or a mixture of $60 \%$ hexane and $40 \%$ methanol. These results again demonstrate that solvents which are more polar are more effective in extracting the lipids out of the sewage sludge. The experiment was finished by evaporation on the rotovap at $40^{\circ} \mathrm{C}$ as describe previously.

\section{Comparison of Solvents on the Types of Lipids Extracted}

Two solvents were compared to determine if there was a difference in the types of lipids extracted by examining the saturated, monounsaturated, and polyunsaturated FAME profile of each. The two extraction techniques investigated were a traditional liquid solvent system comprised of $70 \%$ hexane/30\% methanol and the other was SC$\mathrm{CO}_{2}$. For comparison purposes a profile of soybean and canola as well as the FAME profile of the sewage sludge using the two extraction techniques are provided in Fig. 1.

There was a difference in the types of lipids extracted by these different solvents. The $\mathrm{SC}-\mathrm{CO}_{2}$ solvent was more efficient in extracting the polyunsaturated compared to the $70 \%$ hexane/30\% methanol solvent mixture. It also showed a higher yield on the monounsaturated extracted. Overall the lipids extracted by $\mathrm{SC}-\mathrm{CO}_{2}$ were $78.9 \%$ unsaturated compared to the $49.9 \%$ unsaturated fatty acids extracted by the $70 \%$ hexane/30\% methanol solvent mixture. The fatty acids extracted from the sewage sludge using the $\mathrm{SC}-\mathrm{CO}_{2}$ were closer to the oil profiles traditionally seen with soybean and canola in terms of saturated vs. unsaturated. The more traditional solvent extraction system was more efficient at removing lipids that contained saturated fatty acids.

\section{Comparison of Solvents on the Types of Fatty Acids Extracted}

Since there was a dramatic difference in the amounts of saturated vs. unsaturated fatty acids extracted, the question arises as to the kinds of fatty acids that 
are extracted. The results of the fatty acid analysis are presented in Figure 2. What is obvious right away is the different fatty acid that extracted using the $70 \%$ hexane/30\% methanol solvent mixture vs. the SC-CO2 solvent. The $70 \%$ hexane/30\% methanol extracted more lipids containing C14:0, C14:1, C16:1, C18:0, and C20:1 and higher. The SC-CO2 solvent extracted lipids that had fatty acid profiles more closely similar to those found in soy and canola oil.

\section{SUMMARY}

The work presented here demonstrates the potential to use sewage sludge lipids as a feedstock for biodiesel. Since the types of lipids present in sewage sludges differs from those found in soy and canola oil, different extraction techniques must be identified to maximize lipid yield for biodiesel production. This work has shown that the more nonpolar solvents were more effective than traditional polar solvent such as hexane at extracting the lipids found in sewage sludges.

Two solvents, $70 \%$ hexane $/ 30 \%$ methanol solvent mixture and $\mathrm{SC}-\mathrm{CO}_{2}$ solvent, were investigated further for lipid extraction from sewage sludge. Based on the degree of saturation in the fatty acids extracted it was apparent that different lipids were extracted. The $\mathrm{SC}-\mathrm{CO}_{2}$ extracted lipids that had fatty acid more similar to those fatty acids associated with soy and canola and may suggest that a biodiesel produced from these lipids could have physical properties similar to biodiesel produced from either soy or canola.

With sewage sludge disposal becoming increasing difficult worldwide biodiesel production from this source would reduce the amount of sludge to be handled. The remainder left behind after lipid extraction would essentially be pathogen free due in large part to the lysis of most all-living cells. The residuals from the process should be easier to digest.

Preliminary economics analysis of the process shows that the process could produce a fuel at approximately $\$ 1.85 / \mathrm{gal}$ which compares nicely to the cost of soy biodiesel. With improved extraction and reaction technologies the price could potentially be reduced to approximately $\$ 1.40 /$ gal. 


\section{REFERENCES}

1. Christie, W.W., Preparation of ester derivatives of fatty acids for chromatographic analysis. Advances in Lipid Methodology. V2, 69-111. 1993. Ref Type: Journal (Full)

2. Conrad. A., Kontro, M., Keinänen, M. M., Cadoret, A., Faure, P., Mansuy-Huault, L., and Block, J. 2003. Fatty acids of lipid fractions in extracellular polymeric substances of activated sludge flocs. Lipids, Vol. 38. No.10 pg. 1093-1105.

3. Haas, M. J., McAloon, A. J., Yee, W. C., Foglia, T. A. 2006. A process model to estimate biodiesel production costs. Bioresource Tech. Vol. 97 pg. 671-678.

4. Firestone, David. Physical and Chemical Characteristics of Oils, Fats, and Waxes. 


\section{FIGURES}

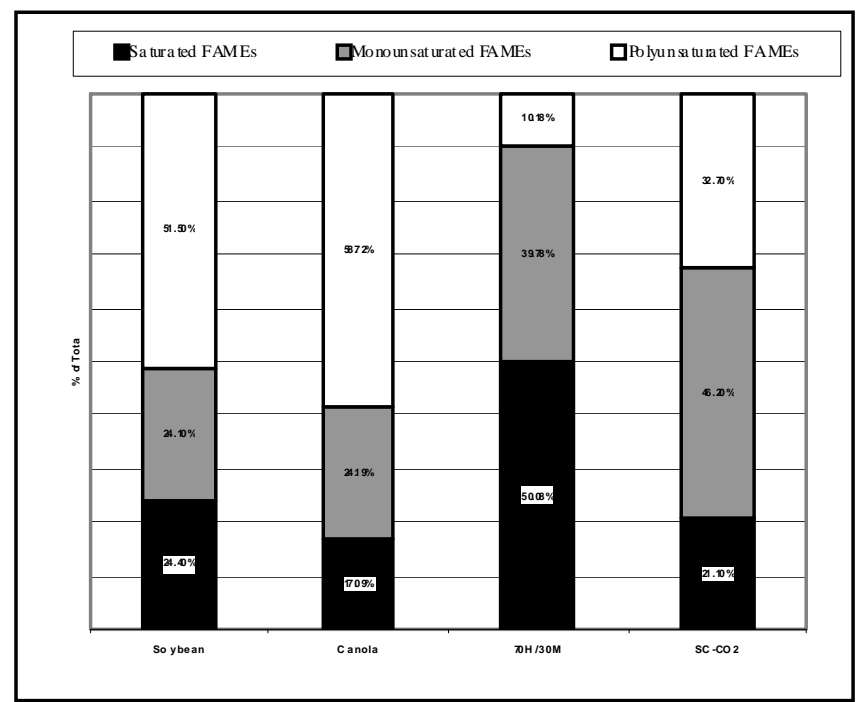

Figure 1. Comparison of the fatty acid extraction by two different solvent systems.

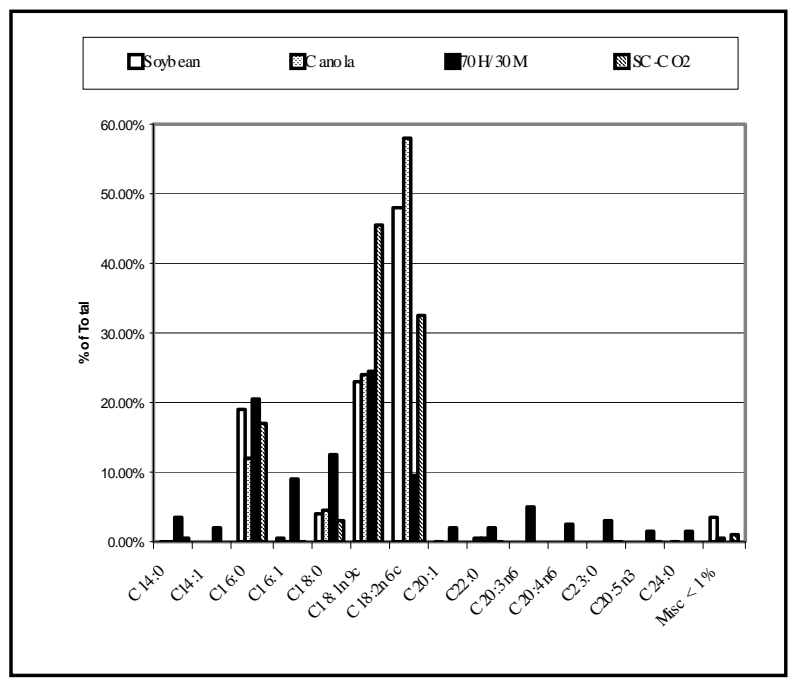

Figure 2. Fatty Acid Profile of Extraction Method 


\section{TABLES}

Table 1. Evaluation of different solvents on oil yield using sewage sludge from the Starkville, MS wastewater treatment facility.

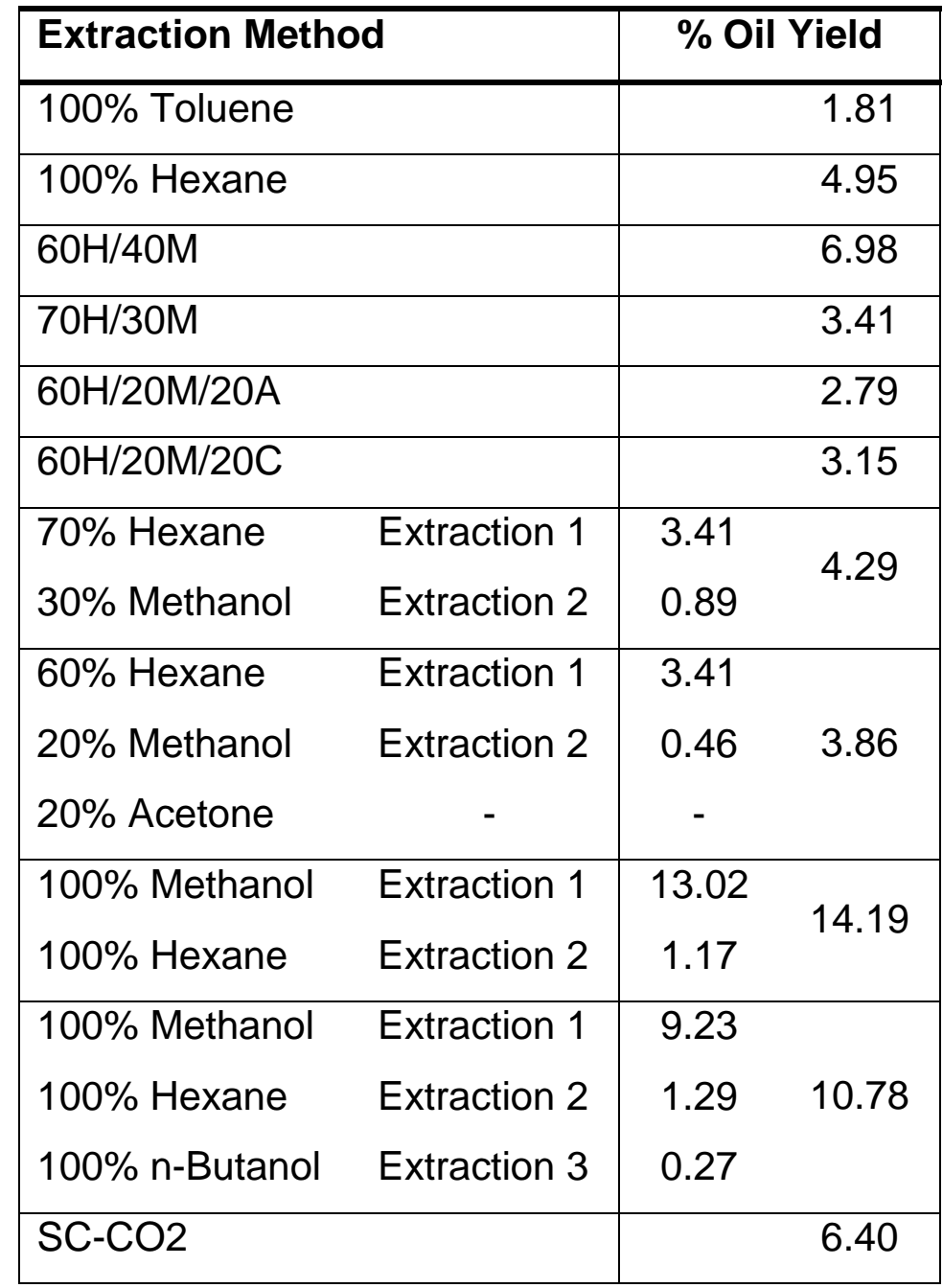


Table 2. Effects of sonication to increase oil yield by freeing bound lipids.

\begin{tabular}{|l|c|}
\hline \multicolumn{1}{|c|}{ Solvents } & Lipid Yield (wt.\%) \\
\hline $100 \mathrm{H}$ & $1.34 \pm 0.49$ \\
\hline $60 \mathrm{H} / 40 \mathrm{M}$ & $3.29 \pm 0.27$ \\
\hline
\end{tabular}




\section{ENZYMATIC CONVERSION OF LIGNOCELLULOSE \\ Performer: University of Mississippi}

PROBLEM STATEMENT

Efficient conversion of lignocellulose requires pretreatment to "free" cellulose from lignin, enhancing conversion rate and yield. Conventional pretreatments use steam and acid, requiring energy inputs, corrosion resistant materials, and neutralization. Enzymatic pretreatment may reduce costs while enhancing conversion and facilitating conversion of lignin to value-added products. For example, relevant biopulping research has shown reduced chemical use for paper making. Challenges to enzymatic pretreatment include bacterial/fungal contamination and metabolism of compounds that should be preserved for conversion to commercial products.

\section{BACKGROUND}

Lignin, due to its intimate association with cellulose and hemicellulose, forms a barrier to enzymatic attack. Bacterial lignin degradation has been reported to be more specific than with fungal systems, an advantage leading to many industrial applications like vanillin, adhesives, binder for laminated or composite wood products, etc. Lignin is a complex biopolymer and small differences in its isolation techniques can result in significant changes in its molecular structure and configuration. Lignin conversion involves complex reactions of bond scission and functional group alteration. It is important to track and quantify these alterations [3-4].

Extensive research has focused on fungal degradation of lignocellulose. However, fungi require high oxygen concentrations and use ligninases (peroxidases) that generate high valence state manganese ions as the nonspecific agent for disrupting the lignin polymer. Little work has been done on other than fungal sources.

For insects, the utilization of lignocellulosic materials for carbon and energy involves a bacterial consortium, often with very different physiological and phylogenetic types. The rate or the fate of the degradation without the activity of the consortium would be limited or absent. Many insects specialize in the degradation of lignocellulosic materials. Most research has focused on the wood-eating termites, and has recently shown that a substantial part of the termite gut is aerobic- or microaerophilic. This 
explains the possibility of the contribution of oxygenases in the degradation of the lignin matrix. A portion of the gut is "anaerobic" as well--so the gut could be considered a gradient oxidation-reduction potential environment [5].

We further understand that the gut is dominated by bacteria, and protozoa (often with bacterial symbionts). The presence of fungi is greatly reduced. Several organisms have been isolated that have the potential to catabolize key functional groups in the dismutation of the lignin superstructure. A "sharing" of catabolic potential is much more energetically beneficial and contributes to process stability. Aspects of the aerobic as well as the anaerobic carbon cycle are most probably critical to the termites' survival [2] [6].

DOE-NREL has supported research with the University of Minnesota on lignin depolymerization by the fungi $T$. Cingulata. Results had shown some depolymerization followed by repolymerization. Recent research has sought to confirm this and to generate enough of the enzymatic protein for genetic analysis. Sophisticated separations technologies involving size exclusion chromatography and light scattering photometry have been used to assay products.

The versatile catalytic activities of chloroperoxidaes (CPO) coupled with the increasing current demands for chiral synthesis have attracted great interests in studying the structural properties of this enzyme, especially the mechanisms through which substrates are enantioselectively transformed into products. Despite the extensive biochemical and biophysical studies carried out, the structural basis for CPO catalyzed enantioselective reactions remain to be delineated. A long-term goal of our research is to unravel the structure function relationship of heme proteins and to elucidate the mechanisms of this class of enzymes at molecular level.

\section{OBJECTIVE AND SCOPE}

The purpose of this research is to understand and enable microbial conditioning to enhance lignocellulose conversion to ethanol and usable lignin co-products. Specific objectives included:

1. investigation of lesser explored sources of lignin depolymerase activity found in gut ecology; 
2. identification of functional consortia of organisms for efficient depolymerization and disengagement of lignin from cellulose;

3. determination of the relation of redox conditions to the extent and nature of organism activity and generation of enzymes;

4. development of functional assays for lignin degradation and multiple levels of assays to screen candidate organisms (Archibald 1992); and

5. development of analytical methods to assess conversion effects on lignin molecular weight, functional groups, and agglomeration characteristics.

In brief, this work explored alternate sources of enzymes from insect and invertebrate gut ecology. Enrichments were performed to obtain functional consortia. These were evaluated for depolymerization of lignin and modification of corn stover. Assay techniques were used to screen for enzymatic conversion rate. Depolymerization was assessed using chromatographic separation and detector systems (RI, UV light scattering).

\section{Specific Tasks}

Tasks specific to this project included:

1. Collection of sources of bacterial enzymes for gut -ecology study, e.g. termites and other wood-eating insects possibly including beetles and wood cockroaches.

2. Development of an analytical technique for tracking lignin depolymerization using AFM and NIR along with HPLC.

3. Incubation screening of sources for lignin depolymerization, from various wood eating insect guts, soils, lignin degrading waste water treatment facility etc.

4. Set-up a weight loss protocol (based on Novozymes protocol) for measuring simultaneous saccharification and fermentation of corn stover.

5. Investigation of beetle gut ecology for possible lignin depolymerization and corn stover utilization.

6. Review of biopulping and solid-state fermentation technologies to support pilotscale test. Make a first-pass effort at bench-scale application of promising organisms and conditions exploiting the expected wet storage scenario for agricultural wastes.

\section{Activities in Concert with DOE-NREL}

Dr. Gidh, our graduate research assistant 2000-2005 completed a six-month internship at NREL. She performed analytical method development for the characterization of lignin using high-pressure size exclusion chromatography (HPLC), refractive index, UV detection and multi angle laser light scattering. This novel method 
is far more reliable than the size exclusion column (SEC-UV detector) used to date. The method was then used to track molecular weight, radius of gyration and hydrodynamic radius changes during fungal (Trametes Cingulata and Phanerochaete Chrysosporium) induced de-polymerization of lignin. This work represents the state of the art. Previous analytical methods required 24 hours to determine the molecular weight distribution. The methods developed can perform this in about 45 minutes.

\section{Accomplishments}

1. Purification and characterization of lignin from kraft black liquor (Mw, rh, rg, $\mathrm{A} 2$, molecular conformation, functional groups)

2. Developed novel applications of HPLC, AFM, NIR for lignin analysis

3. Carried out screenings for lignin depolymerization

4. Analyzed lignin with these tools to validate and understand the depolymerization

5. Isolated 2 bacterial candidates that depolymerized lignin, isolated, identified morphology, and characterized them with biolog

6. Applied the 2 bacteria to PCS to measure improvement in ethanol yield

7. Extended work to corn stover-EtOH production, weight loss, HPLC sugars \& ethanol

\section{Observations and Results}

1. Observed lignin depolymerization with waste water treatment sludge. Data validated on HPLC, AFM and NIR.

2. Isolated 2 bacterial strains from the sludge (white and off-white gram positive rods).

3. Isolated 14 different strains from Betsy beetle guts. The gut contents were evaluated by molecular analysis to give the relative abundance of bacteria. A consortia comprised of these strains showed small but measurable weight loss for corn stover.

\section{Analytical Characterization of Lignin Depolymerization by Fungal and Bacterial Agents}

An analytical method using high-performance size-exclusion chromatography (HPSEC), coupled with Multi-angle Laser Light Scattering (MALS), Quasi-elastic Light Scattering (QELS), Ultraviolet (UV), and Interferometric Refractometry (RI) detection, was developed to characterize and monitor lignin. A Shodex Asahipak GS-320 HQ column with $\mathrm{O} .1 \mathrm{M} \mathrm{NaOH}(\mathrm{pH} 12.0)$ run at $4.5 \mathrm{ml} /$ minute gave the highest separation and most consistent recovery. Zimm, Debye and Berry formalisms were used to 
generate Zimm plots to evaluate the weight average molecular weight (MW), radius of gyration ( $\mathrm{rg}$ ), second virial coefficient (A2) and hydrodynamic radius $(r,$,$) . This study also$ highlighted the differences between the unlyophilized and lyophilized lignin in terms of aggregation, $\mathrm{pH}$ dependence and stability over time. This aggregation has never been seen on a UV detector that has been used so far with SEC, reducing the reliability of depolymerization data obtained without light scattering. Atomic Force Microscopy (AFM) and Near Infrared Spectroscopy (NIR) also proved effective for lignin characterization.

Culture broths from Phanerochaete chrysosporium and Trametes cingulata, combined with cofactors such as hydrogen peroxide, dithiothreitol, copper, iron, and manganese ions were examined for the ability to modify lignin structure. Results showed changes in the molecular weight distribution of lignin components when treated with a combination of factors. The induced cultures showed more lignin depolymerization for the specific lignin samples in which they were initially grown. The distribution in the radius of gyration became narrower with time, indicating that molecular conformation changed to a more uniform molecular shape. H202 and DTT showed the most significant changes in lignin molecular weight distribution

Termites, beetles and other arthropods can digest living and decaying wood and other lignocellulosic plant litter. Microbial sources like other wood eating insect guts and waste water treatment sludge were screened for lignin depolymerization. NIR and AFM along with HP-SEC, were used to track changes in functional groups, size, shape and molecular weight of lignin molecules during incubations. Odontotaenius disjunctus (Betsy beetle) guts dissected whole or separately as mid, fore and hindgut, consumed corn stover but did not show lignin depolymerization. The sludge treated lignin did show some reduction in molecular weight on the HPLC, particle size (350-650 nm initially to 135-220 nm by day 30) and particles per field on AFM. pH and the presence of nutrients had a substantial effect on the extent of depolymerization. Cultures in lignin and nutrients showed higher growth than cultures with lignin only. Colony characteristics within the beetle gut and the sludge were also evaluated using Biolog and Gram Staining.

In addition, the beetle gut bacteria were examined by molecular techniques as the basis for Dinesh Talreja's Ph.D. dissertation research (University of Mississippi, 
Department of Biology, major professor, Al Mikell). The following summarizes the background for symbionts, the value of molecular techniques, our methods, and a figure showing observed relative populations for Betsy beetle bacteria.

\section{Molecular Analysis of Betsy Beetle Gut Flora}

Microorganisms play an important and often essential role in the growth and development of many insect species. Endosymbionts contribute to insect reproduction, digestion, nutrition and pheromone production. Symbiosis generally means close association of different organisms. Of the various symbiotic associations, the most cohesive forms are found in "endosymbiosis", in which one partner, the symbiont, lives inside the body of another partner, the host. Insects feeding on restricted diets, such as plant sap, vertebrate blood, or woody material, often possess symbiotic microorganisms. These microorganisms are always found in the insect in the lumen of the host gut, are vertically transmitted from generation to generation, and usually cannot be cultured outside the bodies of the hosts. The host insects often suffer retarded growth, sterility, and/or death when they are experimentally deprived of their symbionts. Methods for enumerating gut bacteria based on cultivation, isolation, and biochemical testing are generally laborious and do not guarantee recovery of less easily cultivated species. This is a particular problem for obligately anaerobic bacteria, which make up the great majority of organisms present in densely populated gut habitats such as the rumen and the hindgut. For this reason, there has been increasing interest in the rapid enumeration of microbial groupings by analysis of nucleic acids extracted from gut samples. Cultivation-independent molecular techniques have illuminated the enormous microbial diversity that exists on our planet. Saprophagus macroinvertebrates, such as earthworms, termites, and many coleopteran and dipteran larvae, play a major role in the degradation and stabilization of soil organic matter (SOM) and strongly influence important physical and chemical soil parameters. To elucidate the contribution of gut microorganisms to digestion of SOM and host nutrition, information about structure and function of the microbial gut community has to be linked to gut morphology, the physicochemical conditions in different gut compartments, and the spatial distribution of the microorganisms. Due to difficulties in simulating the microenvironment of the insect gut system, culture based approaches do not adequately reflect the microbial diversity 
in the guts of soil feeders; thus it is necessary to study the structure of microbial gut communities with culture-independent techniques.

The application of molecular detection methods has revolutionized the analysis of the diversity of the microbial world. An illustrative example was the observation that a then-unknown and uncultured marine bacterium, SAR11, whose 16S rDNA was first detected in the Sargasso Sea, proved to be one of the most abundant micro-organisms on Earth. The picture of soil bacterial diversity has been changed to a comparable extent. Still, recent work also suggests that biases in widely used procedures might lead to an overestimation of bacterial biodiversity by a factor of 2.4 , highlighting the need to reflect on methodological shortcomings. First of all, one pitfall common to each polymerase chain reaction (PCR)-based technique is an incomplete or biased extraction of DNA from soil samples, depending on bacterial size or cell wall structure. Still, some extraction protocols have found extraction efficiencies in the order of $90 \%$ and little bias towards gram negative bacteria. Second, possible mismatches between the primers used to target $16 \mathrm{~S}$ rDNA and the template DNA would lead to a decrease in PCR efficiency.

"Universal" primers are a prerequisite for a proportionate detection of all templates. Phylogeny is defined as the evolutionary development and history of a species or higher taxonomic grouping of organisms. Insects are the most-species rich group of organisms and it has been estimated that at least $15-20 \%$ of all insects live in symbiotic relationships with bacteria. The early establishment of symbiotic association between insect and bacteria about 300mya, together with nutritional enrichment that bacteria offer to insects, could be the key factors in the evolutionary success of this group of organisms. Insect endosymbionts live in a very close environment, inside specialized host cells called bacteriocytes, which may form an organ-like structure (bacteriome) in the body cavity of insects. The association is obligate for both partners: the bacteria cannot be cultured outside the host, whereas the host needs the bacteria for normal growth and reproduction. The best investigated example is the bacteriocyte symbiosis between aphids and Buchnera aphidoicola, but similar endosymbiotic relationships have also been described in many other insect families, including weevils, mealybugs, whiteflies, tsetse flies, psyllids, leafhoppers, carpenter ants and 
cockroaches. An extreme case of intracellular symbiosis has been reported in some mealybugs, in which the bacteriocytes contain $\beta$-proteobacterial endosymbionts that are themselves colonized by intracellular $\mathrm{y}$-proteobacteria. Molecular phylogenetic studies based on $16 \mathrm{~S}$ rDNA sequences revealed phylogenetic congruence of these primary endosymbiotic bacteria with their hosts. All these studies span ancient divergence among taxa, indicating that each symbiosis is the result of a single bacterial infection of the insect ancestor, which was followed by co-evolution of both partners across millions of years. For these studies microbial diversity from the gut of Odontotaneous disjunctus (Betsy beetle) was estimated using both culturable and molecular techniques (Figure 1).

DNA was obtained from the whole gut of Betsy beetles. It was amplified using polymerase chain reaction and cloned using TOPO TA cloning ${ }^{\circledR}$ Kit (containing pCR $\circledast 2.1-T O P O \circledR)$ with Top10F' OneShot $\circledast$ Chemically Competent cells. Colonies were picked from Ampicillin plates and sequenced to estimate the diversity of bacteria in the beetle gut. The latter was conducted by a research group at Oklahoma University Health Science Center (OUHSC) Oklahoma City, OK. The primary results obtained from the whole gut of Betsy beetle are given in Figure 2. 


\section{REFERENCES}

1. Archibald, F.S., 1992, A new assay for lignin-type peroxidases employing the dye azure B, Applied and Environmental Microbiology, 1992 58, 3110-3116

2. Brune, A., Miambi, E., and Breznak, J.A. (1995). Roles of oxygen and the intestinal microflora in the metabolism of lignin-derived phenylpropanoids and other monoaromatic compounds by termites, Appl. Environ. Microbiol. 61:2688-2695.

3. Jensen Jr., K. A.; Bao, W.; Kawai, S.; Srebotnik, E., and Hammel, K. E. (1997). Manganese-dependent cleavage of nonphenolic lignin structure by Ceriporiopsis subvermispora in the absence of lignin peroxidase, Applied and Environmental Microbiology, $\underline{62}$ 3679-3686.

4. Sonoki, T., Obi, T., Kubota, S., Higashi, M., Masai, E., Katayama, Y. (2000). Genetics and Molecular Biology - Coexistence of two different o demethylation systems in lignin metabolism by sphingomonas paucimobilis SYK-6: cloning and sequencing of the lignin biphenyl-specific o-demethylase (LigX) gene, Applied and Environmental Microbiology, 2000 - Volume 66 - Issue 5.

5. Tokuda, G.; Watanabe, H.; Matsumoto, T.; and Noda, H. 1997, Cellulose digestion in the wood-eating higher termite, Nasutitermes takasagoensis, Zoological Science, 14 , no. $1,83-93$.

6. Zimmerman, W., 1990, Degradation of Lignin by Bacteria, Journal of Biotechnology, 13, no. 2-3, 119-130. 


\section{FIGURES}
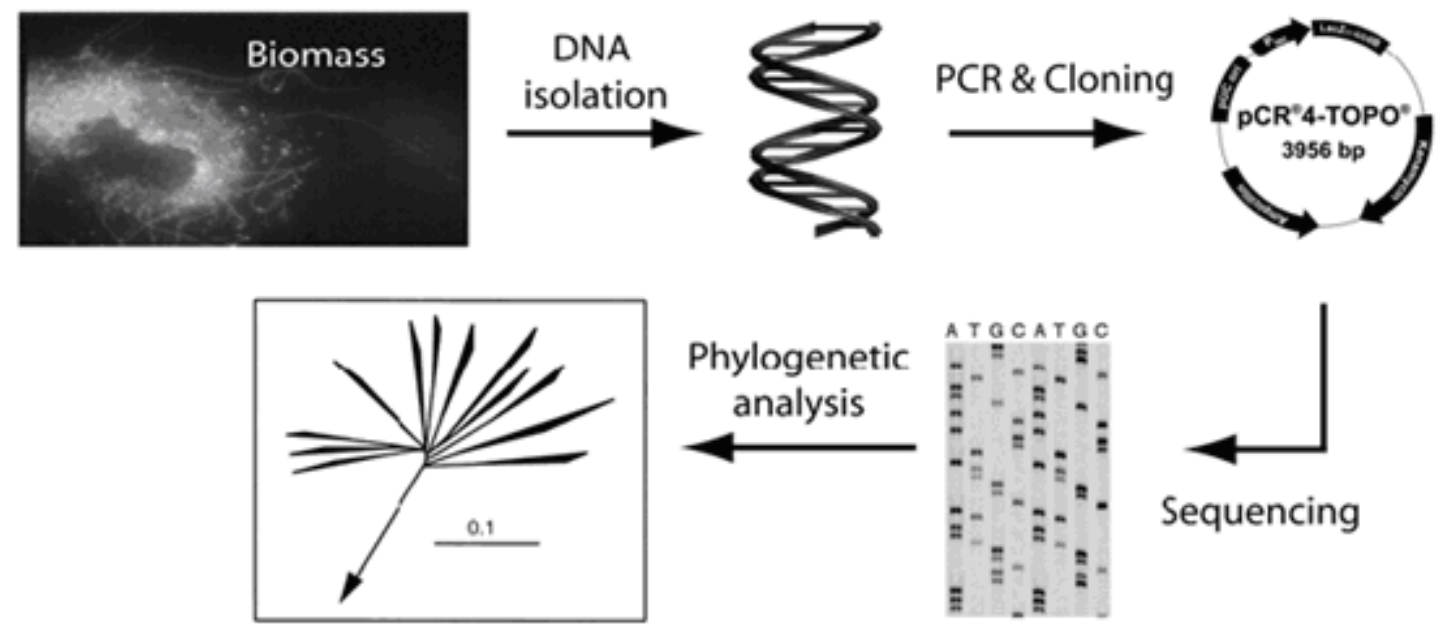

Figure1: Steps involved in estimating prokaryotic diversity from the whole gut tissue of Betsy beetle Odontotaenus disjunctus) 


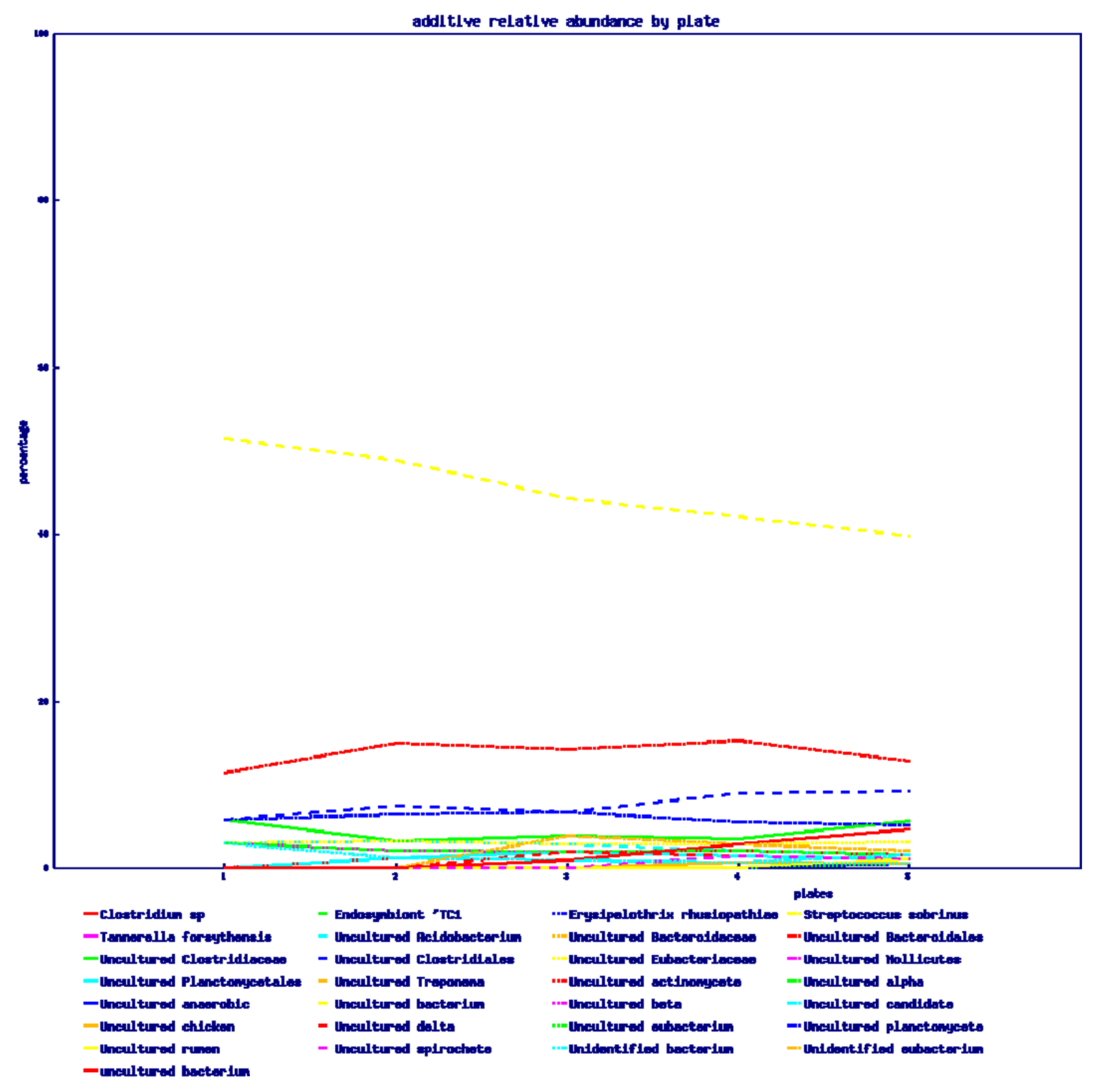

Figure 2: Additive relative abundance of different bacterial population 


\section{DEVELOPMENT AND COMPARISONS OF EFFICIENT GAS-CULTIVATION SYSTEMS FOR ANAEROBIC CARBON MONOXIDE UTILIZING ORGANISMS Performer: Mississippi State University}

\section{INTRODUCTION}

Anaerobic microorganisms that utilize gaseous compounds such as $\mathrm{CO}, \mathrm{CO}_{2}$, and $\mathrm{H}_{2}$ as their source of carbon and energy are known as acetogens and methanogens. The cultivation of these gaseous utilizing-microorganisms is traditionally carried out by either displacing the headspace above or bubbling gases through the medium in a fixed volume culture vessel such as test tubes or flask and stoppered with a butyl-rubber stopper, as was the case with Ohwarki [6], Svetlitchnyi [9], Sokolova [8]. A technique similar to the one used here was described by Kafkewitz [4] for the cultivation of $\mathrm{H}_{2}$ producing Ruminococcus albus. In their study a chemostat was modified to include a football bladder for the collection of the $\mathrm{H}_{2}$ produced. Cultivating microorganisms in this fashion requires a great deal of safety precautions especially when $\mathrm{CO}$, a toxic substance, or $\mathrm{H}_{2}$, a flammable substance, is the gas being sparged through the reactors. Henstra and Stams [3] cultivated their CO-utilizing microorganisms, Carboxydothermus hydrogenformans and Thermoterrabacterium ferrireducens, using $120 \mathrm{~mL}$ bottles stoppered with butyl rubber and pressurized to $\sim 1.7$ atms with a mixture of $\mathrm{CO}_{2}$ and either $\mathrm{CO}, \mathrm{N}_{2}$, or $\mathrm{H}_{2}$.

Cultivation of anaerobic microorganisms requires specialized techniques differing from those used to culture aerobic microorganisms. Obviously, oxygen in the system must either be reduced to a level acceptable by target microorganisms or removed from the system. Doan [1] evaluated the Coy chamber, GasPak system, and AnaeroPack system for maintaining anaerobic conditions. Results of this study did not show any appreciable difference in growth of the seven cultures tested. These types of systems work well for cultivation of anaerobic microorganisms that utilize carbon sources that are liquid or solid at room temperature and pressure. However, when a carbon source is in the gaseous form, cultivation of these microorganisms becomes more difficult.

Although these techniques have been successfully used to cultivate many types of microorganisms, there are limitations and concerns associated with these methods. Only a defined amount of substrate can be delivered at a time using fixed volume 
culture vessels with limited pressurizing capacities. Then, as the substrate is transferred into the liquid phase and consumed by microorganisms, its partial pressure decreases, and consequently, the mass transfer rate. Fast reactions could become mass transfer limited as pressure in the culture vessel decreases. Use of mass transfer limited data to calculate reaction kinetics (e.g., reaction order, rate constant, and activation energy) results in incorrect values for industrial scale-up of chemical and biological processes.

A new method of cultivation that operates at atmospheric pressure (safer to handle) and capable of minimizing or eliminating mass transfer limitation would add an important tool to the arsenal of techniques utilized by microbiologists with gas substrates. The system described below utilizes Cali-5-BondTM gas-sampling bags to culture $\mathrm{CO}, \mathrm{CO}_{2}$, and $\mathrm{H}_{2}$ utilizing microorganisms without substrate limitations and/or reduced substrate mass transfer rates experienced with fixed volume vessels. This system also decreases the high cost typically associated with vials sparged with the substrate.

\section{METHODS}

\section{Medium}

All chemicals used in the medium were obtained in technical or laboratory grades from Fisher Scientific (Hampton, New Hampshire) or Sigma-Aldrich (St. Louis, Missouri). The Acetate Production Medium (APM) utilized in this study was prepared as described in a patent by Gaddy and Clausen [2]. After autoclaving, the bottles were placed in an anaerobic glove bag (Coy Laboratory Products) where the medium cooled to room temperature and any dissolved oxygen was removed. Medium was dispensed into culture $40 \mathrm{~mL}$ Fisherbrand EPA vials (Fisher Scientific) using a Wheaton Adjustable Volume Self-Refilling Repetitive Syringe.

\section{Culture Vials}

Fisherbrand $40 \mathrm{~mL}$ EPA vials were used with each of three types of caps: crimp, Si/PTFE, and Mininert ${ }^{\circledR}$. Crimp tops (Fisher Scientific) included Teflon/Butyl stoppers. The Si/PTFE caps (Fisher Scientific) consisted of open-top polypropylene closures with 0.005" PTFE/0.120" silicone rubber septa with the PTFE side of the septum facing into 
the vial. The Mininert ${ }^{\circledR}$ valve caps from $\mathrm{VICl}$ Precision Sampling (Baton Rouge, Louisiana) included silicone needle septa with PTFE faced caps.

\section{Gas Sampling Bags}

Tedlar HandyGrab ${ }^{\mathrm{TM}}$ gas-sampling bags were purchased from Zefon International, Inc. (Ocala, FL). The $0.6 \mathrm{~L}, 2$ mil thick pillows (bags) featured one polypropylene fitting that combines valve and septum. Five mil thick, $0.5 \mathrm{~L}$ capacity Teflon gas-sampling bags were purchased from Fisher Scientific. Each Teflon bag had a septum fitting in a polypropylene housing. Cali-5-Bond ${ }^{\mathrm{TM}}$ gas-sampling bags were purchased from Calibrated Instruments, Inc. (Hawthorne, New York) and are shown in Figure 1. The 5 mil thick, $0.5 \mathrm{~L}$ pillows were equipped with one replaceable septum holder and one twist on/off valve per bag. Si/PTFE septa were used in an all bags with the PTFE side facing the inside of the bag.

\section{Headspace Gas Analysis}

Headspace gases were analyzed using an Agilent 6890N Network Gas Chromatograph System with a Thermal Conductivity Detector and a manual injection port. The system used a column selection method fitted with two Supelco columns (Bellefonte, Pennsylvania): a 45/60 Molecular Sieve 5A (10 ft x 118 in Stainless Steel) and an 80/100 Porapak Q (6ft $\times 1 / 8$ in Stainless Steel). Calibration curves for $\mathrm{N}_{2}, \mathrm{O}_{2}$, and $\mathrm{CO}$ were prepared by analyzing known volumes of pure $\mathrm{N}_{2}, \mathrm{O}_{2}$, and $\mathrm{CO}$ samples purchased from Nexair in Columbus, MS using a Hamilton $® 100 \mu \mathrm{L}$ gas-tight sampling syringe

\section{Vial Cap Testing}

This experiment was conducted to determine which of 3 septum types would perform best with respect to maintaining the headspace gas composition. A $1 \%$ resazurin in water solution was prepared for use as an oxygen indicator in this experiment. Resazurin solution reacts with oxygen, causing the solution color to change from colorless to pink when low levels of oxygen are present. Simultaneously boiling and sparging nitrogen into the solution prior to the experiment removed dissolved oxygen. Six vials were prepared using each of three types of vial cap: crimp tops, Si/PTFE screw caps, and Mininert $₫$ valve screw caps. Crimp tops with Teflon/butyl 
rubber stoppers were used with matching $20 \mathrm{~mL}$ vials, while the other caps were used with $40 \mathrm{~mL}$ vials. Vials were filled halfway with $1 \%$ resazurin solution $(10 \mathrm{~mL}$ for crimp vials, $20 \mathrm{~mL}$ for others) in the anaerobic glove bag. All vials were capped with the appropriate tops and removed from the glove bag for gassing.

The vials with the septum materials to be evaluated were gassed with $100 \%$ CO using the technique described below. Gases were introduced into the vials using a sterile gassing syringe. The gassing syringe consisted of a pre-sterilized 10-cc glass syringe stuffed with glass wool with a rubber stopper on the plunger end. A glass tube inserted through a hole in the stopper connected the syringe to the tube leaving the gas mixing apparatus.

All gassing procedures were performed under a fume hood. Gas was allowed to flow into the sealed vial for $10-15$ seconds to create a slightly positive pressure inside the vial and prevent oxygen from entering the headspace. At that time, the vial cap was loosened to purge the headspace of nitrogen. Gas flowed continuously into the vial for 2 minutes, and then the vial cap was tightened for the final 10-15 seconds to create a slight positive pressure within the vial headspace.

Three vials for each cap type were placed in the shaker incubator at $37^{\circ} \mathrm{C}$ and shaken at $100 \mathrm{rpm}$. Three additional vials for each cap type were incubated statically at $37^{\circ} \mathrm{C}$ incubator. Gas samples of all vials were taken every 24 hours for 3 days with one final sampling after 7 days of incubation.

\section{RESULTS}

\section{Vial cap and culture vial evaluations}

Determining the rate of gaseous substrate consumption by microorganisms on a microcosm scale, i.e. less than $100 \mathrm{~mL}$, without the introduction of oxygen through a worn septum or substrate becoming rate limiting can be challenging. To this end a series of experiments was conducted to determine if septa enclosed $40-\mathrm{mL}$ vials could over come these issues.

The first objective was to compare the ability of different septa to prevent loss of substrate and intrusion of oxygen from the atmosphere over time. In this experiment, three septa were evaluated. Teflon/Butyl septa (Figure 2.) performed well in the first 24 hours of the experiment. It should be pointed out that error bars are magnified by the 
short $y$-axis $(2 \%)$. The reason for the short $y$-axis was that using a full scale would not have allowed for viewing differences. However, subsequent headspace samplings at 48, 72 and 168 hours post setup showed a steady increase in the oxygen concentration in the septa enclosed vials. Another septum evaluated was a Si/PTFE rubber septum. From the data, the ability of these septa to maintain anaerobic conditions over 72 hours is clearly demonstrated for both incubation conditions. This type of septum was able to maintain strict anaerobic condition for 168 hours when vials were allowed to remain static. Some oxygen (>1\%) was present in samples taken after 7 days of incubation for those septa enclosed vials shaken at $150 \mathrm{rpm}$. Mininert ${ }^{\circledR}$ tops were the third type of closures evaluated. They outperformed the Teflon/Butyl rubber tops and performed similar to Si/PTFE rubber septum over a 3 day incubation period independent of the incubation conditions, i.e. shaken at $150 \mathrm{rpm}$ or static. Headspace analysis on the vials closed with Mininert $®$ tops after 168 hours of incubation revealed oxygen concentrations in these culture vessels had only changed approximately $1 \%$ for those vessels incubated statically or shaken.

In addition to $40-\mathrm{mL}$ vials and cap types, 2 mil and 5 mil gas-sampling bags manufactured from Teflon, and $0.5 \mathrm{~L}, 5$ mil Cali-5-Bond gas-sampling bags were evaluated. Neither of the gas-sampling bags manufactured from Teflon were able to maintain the $\mathrm{CO}$ concentration for 24 hours and were therefore removed from any further evaluations. The 5 mil gas-sampling bags outperformed all of the vials tested using static incubating conditions. Gas-sampling bags when shaken, demonstrated an increase in oxygen content in as little as 24 hours. However, oxygen levels remained at the 24 hour level for the next 48 hours as shown in the 72 hour analysis. The 168 hours headspace analysis revealed a slight increase in oxygen but the overall change in oxygen in the headspace was less than $1 \%$.

Effects of incubating conditions can be seen from the data presented in Figure 2. The $40 \mathrm{~mL}$ vials sealed with the Si/PTFE rubber septum or Mininert ${ }^{\circledR}$ tops and the gassampling bags statically incubated showed no detectable oxygen after 48 hours of incubation and only a trace amount after 72 hours of incubation for those vessels sealed with Mininert $®$ tops. Incubation conditions appear to have little impact on vessels sealed with Teflon/Butyl rubber stoppers after 72 hours of incubation. Although oxygen 
entered the gas-sampling bags that were shaken, these values remained constant after 72 hours of incubation.

Changes in $\mathrm{N}_{2}$ and $\mathrm{CO}$ overtime for these culture vessels were also measured and are presented in Figure 3 and 4. The differences in performance of the septa evaluated become apparent. As before in Figure 2, short y-axis were used to demonstrate differences but give the appearance of large errors. The vials sealed with the Teflon/Butyl rubber and Si/PTFE rubber demonstrated changes in the concentration of $\mathrm{N}_{2}$ and $\mathrm{CO}$ within 48 hours of incubation for both the vials shaken and incubated static. Change in $\mathrm{N}_{2}$ and $\mathrm{CO}$ for $40 \mathrm{~mL}$ vials sealed with Mininert $\circledast$ tops was less than $1 \%$ through 72 hours of incubation. However, headspace analysis after 168 hours of incubation showed that average changes in $\mathrm{N}_{2}$ and $\mathrm{CO}$ ranged between $4-6 \%$ from $95 \%$ for $\mathrm{N}_{2}$ and $5-7 \%$ from $100 \%$ for $\mathrm{CO}$. Variations within these sample sets also increases sharply. On the other hand, gas-sampling bags showed less than a $1 \%$ change in $\mathrm{N}_{2}$ for all sampling times and incubation conditions. Change in $\mathrm{CO}$ was approximately a $1 \%$ change for all sampling times. Those gas-sampling bags that were shaken during incubation had the greatest change in $\mathrm{CO}$ after 24 hours of incubation but maintained the $\mathrm{CO}$ for the duration of the experiment.

\section{Mass Determination}

Unlike highly water-soluble substrates like sugars and peptides, $\mathrm{CO}$ is not very water-soluble and the mass that can be applied is limited by the headspace of the culture vessel. For example, a $40 \mathrm{~mL}$ vial with $20 \mathrm{~mL}$ of cell suspension is limited to 20 $\mathrm{mL}$ of $\mathrm{CO}$ at $1 \mathrm{~atm}$ of pressure. Obviously, pressurizing the $40 \mathrm{~mL}$ vials can increase the mass of $\mathrm{CO}$ and its water solubility in these fixed volume vials. Unfortunately, there is little information available as to the amount of pressure that can be safely applied to these types of vials.

Calculations were performed to determine what pressure of $\mathrm{CO}$ would be required in a $40 \mathrm{~mL}$ vial to equal the mass of $\mathrm{CO}$ in a $500 \mathrm{~mL}$ Cali-5-BondTM gassampling bags assuming each had $10 \mathrm{~mL}$ of medium. Results of these calculations are given in Figure 5. Data show that gas-sampling bags contain approximately 6 times

more mass of $C O$ than $40 \mathrm{~mL}$ vials at $1 \mathrm{~atm}$ of pressure. To achieve the same mass of $\mathrm{CO}$ in a vial that was in a gas-sampling bag, the vial would have to be pressurized to 
6.3 atm with CO. Given that no information is available as to whether or not the vials could safely maintain these conditions, it would not be advisable to pressurize these 40 $\mathrm{mL}$ vials significantly above $1 \mathrm{~atm}$. Pressurizing these vials could increase the loss of substrate and reduce the safety of the experiment.

\section{Cultivation of CO-Utilizing Microorganism}

The ability of a gas-sampling bag cultivation system to cultivate CO-utilizing microorganisms was evaluated using Clostridium ljungdahlii (ATCC \# 55383) as the test organism and compared to a traditional vial system using the same microorganism with $\mathrm{CO}$ as the sole carbon and energy source. Results presented in Figure 6 show that those cultures in the gas-sampling bag cultivation system responded much quicker than those in the vials in less than 24 hours as evidenced by the increase in absorbance. No difference was found for cultivation of $\mathrm{C}$. ljungdahlii in the gas sampling bag cultivation system using shake or static conditions. Cultures in the vials showed a slight increase in optical density readings. Absorbance of light at $600 \mathrm{~nm}$ wavelengths was measured for each sample via Spectronic* Genesys* 20 Spectrophotometer (Thermo*Electron) after 24 hours of incubation. As with the gas sampling bag cultivation system, the vial data showed no effect of shaking the culture during incubation verses leaving it static in terms of increased optical density readings. The optical density readings for the gas sampling bag cultivation system were $15 x$ greater than those for the vials after 24 hours of incubation.

\section{DISCUSSION}

Cultivation of microorganisms that utilize gaseous carbon compounds as carbon and/or energy requires different techniques from those used to cultivate microorganisms metabolizing sugars, amino acids, or lipids. The difficulty of these techniques can be even more challenging when the culture requires an anaerobic environment. Often the microorganism's ability to metabolize these gaseous substrates is limited by an inadequate supply of substrate or mass transfer rate of substrate into the aqueous phase. This is due in large part to the types of culture vessels that are traditionally used (i.e. bottles, vials, and flasks). The use of gas-sampling bags is a novel method for cultivating microorganisms that utilize gaseous substrates. This method overcomes 
limited supply of substrate inherent with fixed volume bottles, vials, and flasks and performs as well as other more established techniques maintaining anaerobic conditions. Gas-sampling bags can be purchased in sizes ranging from 0.25 to $1.00 \mathrm{~L}$, which gives flexibility in designing experiments. Depending on the gas-sampling bag design, these systems can be recharged with a gaseous substrate with little effort.

An additional benefit of gas-sampling bags is a larger driving force for substrate transfer from the gas to the liquid phase over the duration of the experiment. In fixed volume vessels, substrate rate of disappearance from the gas phase is given by:

where,

$$
\frac{d C_{A}}{d t}=r_{A}
$$

$$
\begin{aligned}
& C_{A}=\text { Substrate concentration, } \frac{\mathrm{mol}}{\mathrm{L}} \\
& r_{A}=\text { rate of disappearance of substrate } \mathrm{A}, \frac{\mathrm{mol}}{\mathrm{L} \cdot \mathrm{sec}}
\end{aligned}
$$

In contrast, in the sampling bags the substrate rate of disappearance is given by:

$$
r_{A}=\frac{d C_{A}}{d t}+\frac{C_{A}}{V} \frac{d V}{d t}
$$

where,

$$
\mathrm{V}=\text { head space volume, } \mathrm{L}
$$

As can be observed in Equations (1) and (2), the reduction in volume of the gassampling bags causes a faster substrate rate of disappearance. Gas-sampling bag systems a favor kinetically limited condition compared to fixed volume vessels. This condition is critical to measure reliable kinetic parameters. In other words, as microorganisms consume substrate from the gas-sampling bags the volume reduction enhances substrate transfer into solution compared to fixed volume systems.

Sterilization and reuse of gas-sampling bags is an issue that could not be addressed by autoclaving in this study. It was observed that after autoclaving the 5 mil 
gas-sampling bags they were not capable of maintaining a headspace of $\mathrm{CO}$ due to micro-tears in the bags or damages to the seams. Bags could be washed to remove residues from a previous experiment but would need to be sterilized by means other than autoclaving such as ethylene oxide or $y$ radiation.

It should be pointed out that gas-sampling bags manufactured from Teflon used in this study failed to maintain $\mathrm{CO}$ in the bag for 24 hours. The other systems evaluated were the 2 mil Tedlar gas-sampling bags and their performance was determined to be unacceptable. Based on the findings of this study it is suggested that gas-sampling bags manufactured with a thickness of 5 mil or greater be utilized to culture microorganisms on this type of gaseous substrate.

Cultivation experiments using Clostridium ljungdahlii demonstrated the effectiveness of the gas sampling bag cultivation system to cultivate $\mathrm{CO}$ utilizing microorganisms. This system produces a greater concentration of cells in 24 hours of incubation than traditional fixed volume system produced. Wide deviation with the gas sampling bag cultivation system was from one sample at the 24 hours readings with an optical density reading of 0.26 while the other two samples had optical density measurements of 0.104 and 0.078 . A possible explanation to this observation is that a clump of cells was retrieved from the gas sampling bag cultivation system and summarily dispersed prior to taking the optical density reading. In all the optical density readings were $15 x$ greater than those observed for the $C$. ljungdahlii cultivated in the vials. This strongly suggests that the gas-sampling bag cultivation system is superior to traditional vial cultivation system for $\mathrm{C}$. ljungdahlii using $\mathrm{CO}$ as the sole carbon and energy source.

This work has demonstrated the feasibility of using gas-sampling bags as vessels to cultivate gaseous substrate utilizing microorganisms. This system can overcome the supply limitations that are inherent with fixed volume vessels. Operation at atmospheric pressure and the capability of utilizing larger amounts of gas at the initiation of an experiment increases safety, minimizes handling to toxic gases, and enhances the reliability of the measured kinetic parameters. 


\section{REFERENCES}

1. Doan, N., Contreras, A., Flynn, J., Morrison, J., Slots, J., 1999. Proficiencies of three anaerobic culture systems for recovering periodontal pathogenic bacteria. J. Clin. Microbiol. 37, 171-174.

2. Gaddy, J. L., Clausen, E. C., 1992. A clostridium ljungdahlii, an anaerobic ethanol and acetate producing microorganism. US Patent \# 5,173,429, issued on Dec 22, 1992.

3. Henstra, A. M., Stams, A. J. M., 2004. Novel physiological features of carboxydothermus hydrogenoformans and thermoterrabacterium ferrireducens. Appl. and Environ. Microbiol. 70 , 7236-7240.

4. Kafkewitz, D., lannotti, E. L., Wolin, M. J., and Bryant, M. P., 1973. An anaerobic chemostat that permits the collection and measurement of fermentation gases. Appl. and Environ. Microbiol. 25 , 612-614.

5. Maschio, G., Lucchesi, A., Stoppato, G., 1994. Production of syngas from biomass. Bioresource Tech. 48, 119-126.

6. Ohwarki, K. and Hungate, R. E. 1977. Hydrogen utilization by clostridia in sewage sludge. Appl. and Environ. Microbiol. 33 , 1270-1274.

7. Phillips, J. R., Klasson, K. T., Clausen, E. C., Gaddy, J. L., 1993. Biological production of ethanol from coal synthesis gas: medium development studies. Appl. Biochem. and Biotech. 39-40, 559-571.

8. Sokolova, T. G., Kostrikina, N. A., Chernyh, N. A., Tourova, T. P., Kolganova, T. V., and Bonch-Osmolovshaya, E. A., 2002, Carboxydocella thermautotrophica gen. nov., sp. Nov., a novel anaerobic, CO-utilizing thermophile from a Kamchatkan hot spring. Internat. J. of Syst. And Evolut. Microbiol. 52, 1961-1967.

9. Svetlitchnyi, V., Peschel, C., Acker, G., Meyer, O., 2001. Two membraneassociated NiFeS-carbon monoxide dehydrogenases from the anaerobic carbonmonoxide-utilizing eubacterium carboxydothermus hydrogenoformans. J. of Bacteriol. 183, 5134-5144. 


\section{FIGURES}

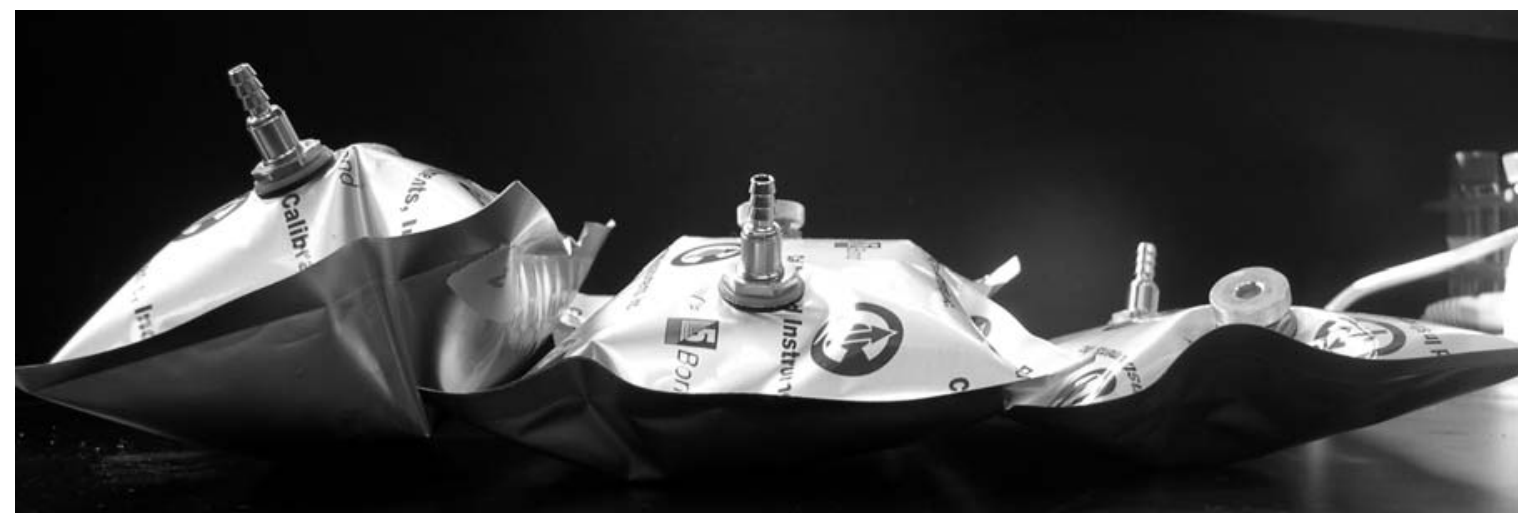

Figure 1. 5 mil thick, $0.5 \mathrm{~L}$ Cali-5-Bond ${ }^{\mathrm{TM}}$ pillows equipped with one replaceable septum holder and one twist on/off valve per bag.

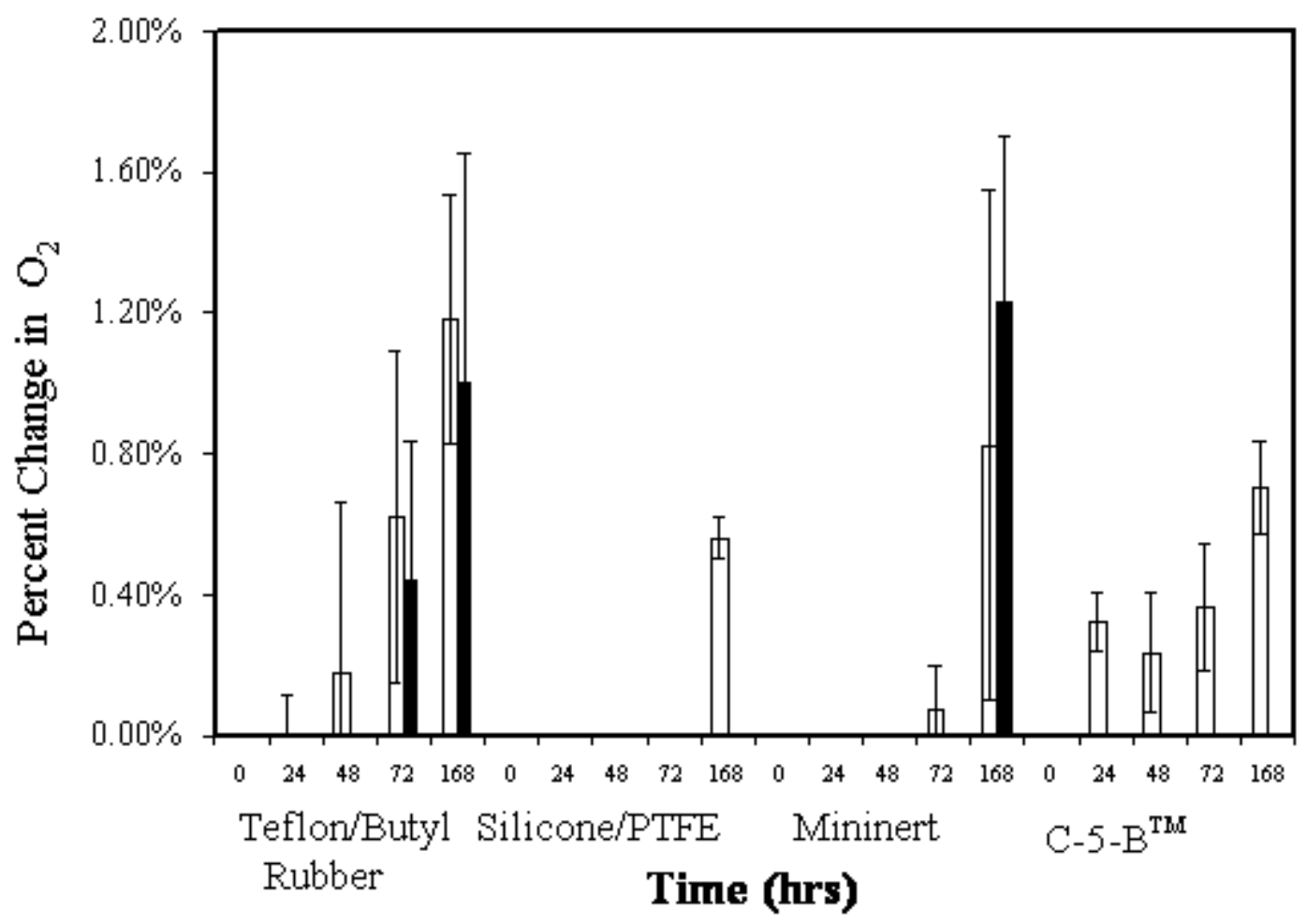

Figure 2. Comparison of four capping materials used to prevent $\mathrm{O}_{2}$ from entering the vessel over $168 \mathrm{~h}$ of incubation. Symbols: black bars, static incubation conditions; white bars, shaken incubation conditions. 


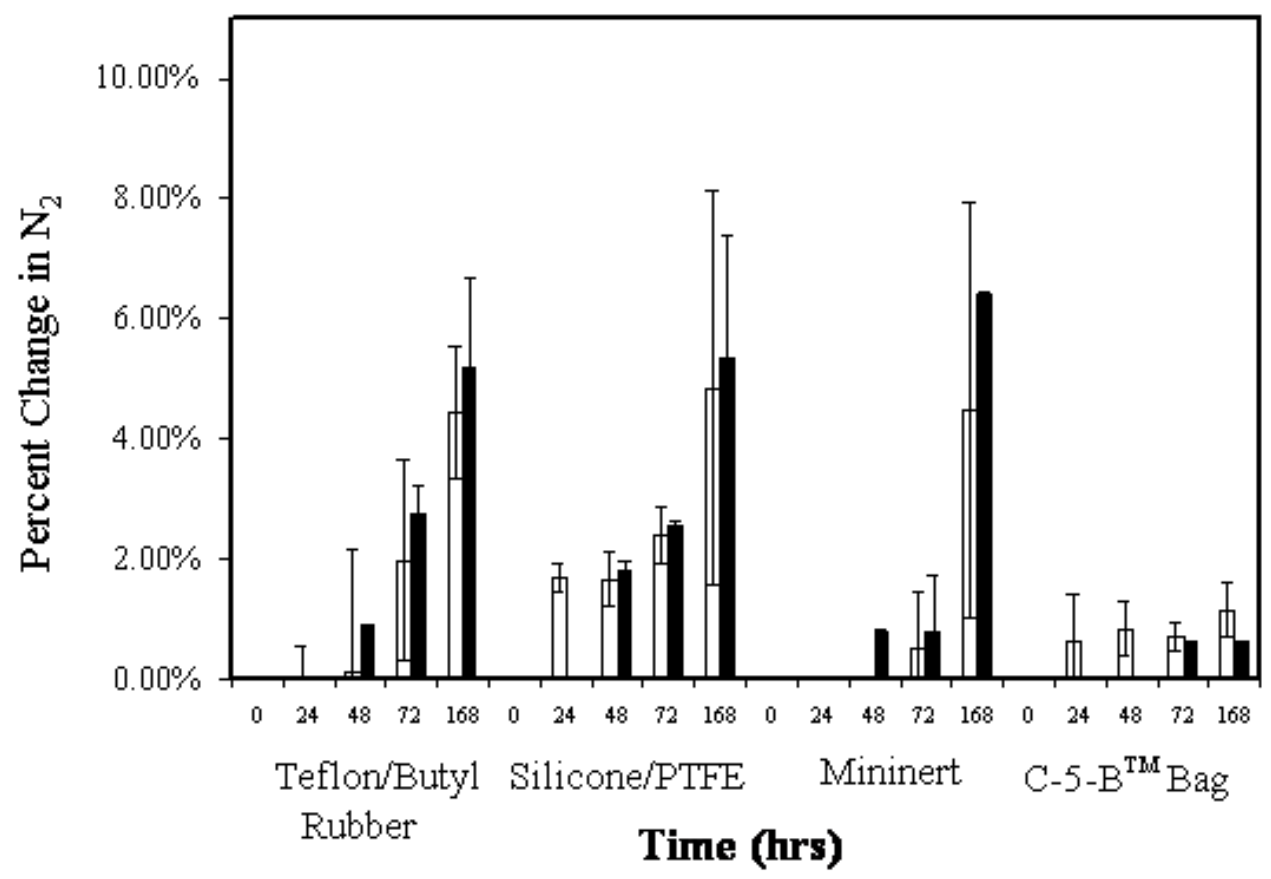

Figure 3. Comparison of four capping materials used to maintain an atmosphere of $\mathrm{N}_{2}$ over $168 \mathrm{~h}$ of incubation. Symbols: black bars, static incubation conditions; white bars, shaken incubation conditions.

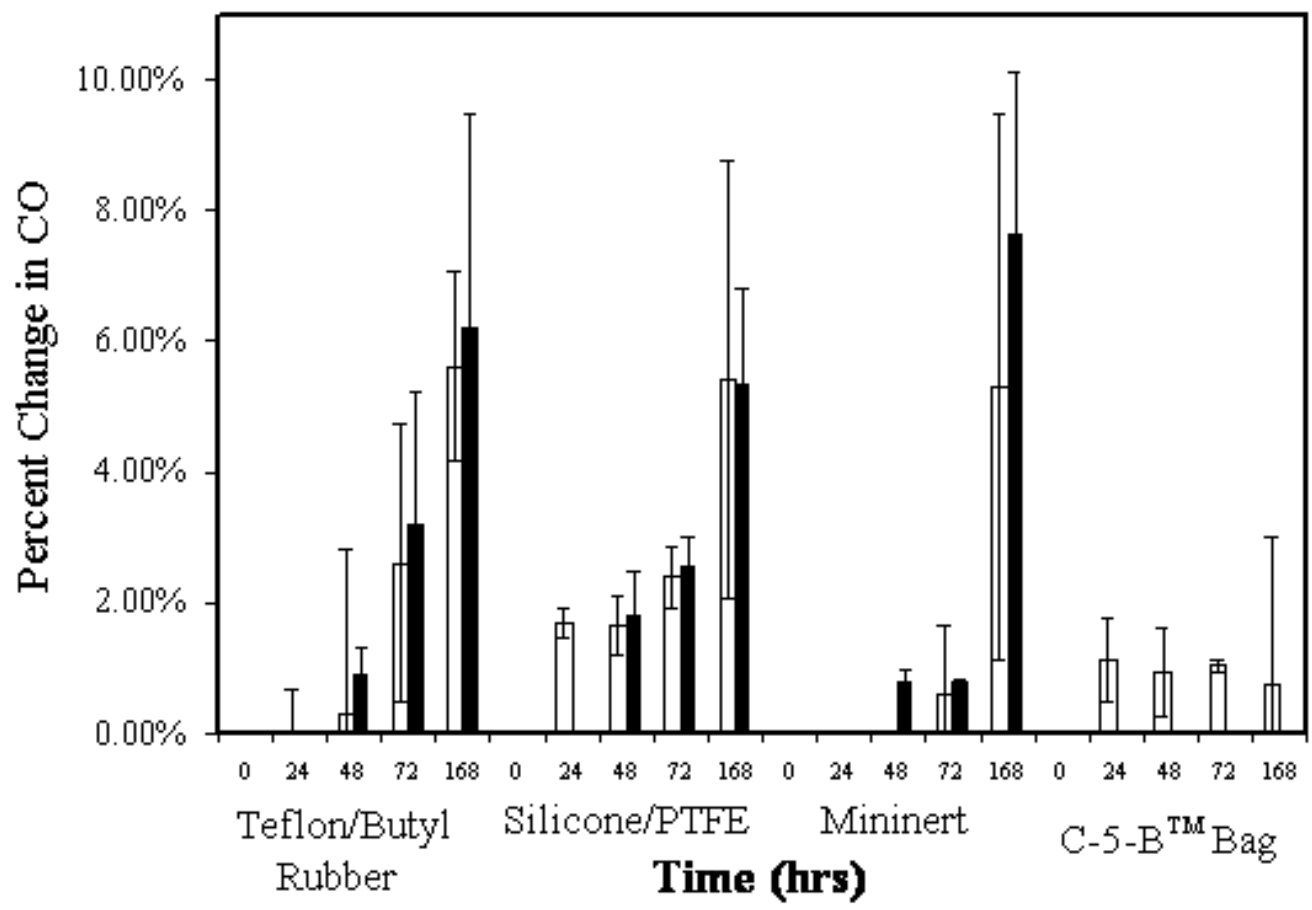

Figure 4. Comparison of four capping materials used to maintain an atmosphere of CO over $168 \mathrm{~h}$ of incubation. Symbols: black bars, static incubation conditions; white bars, shaken incubation conditions. 


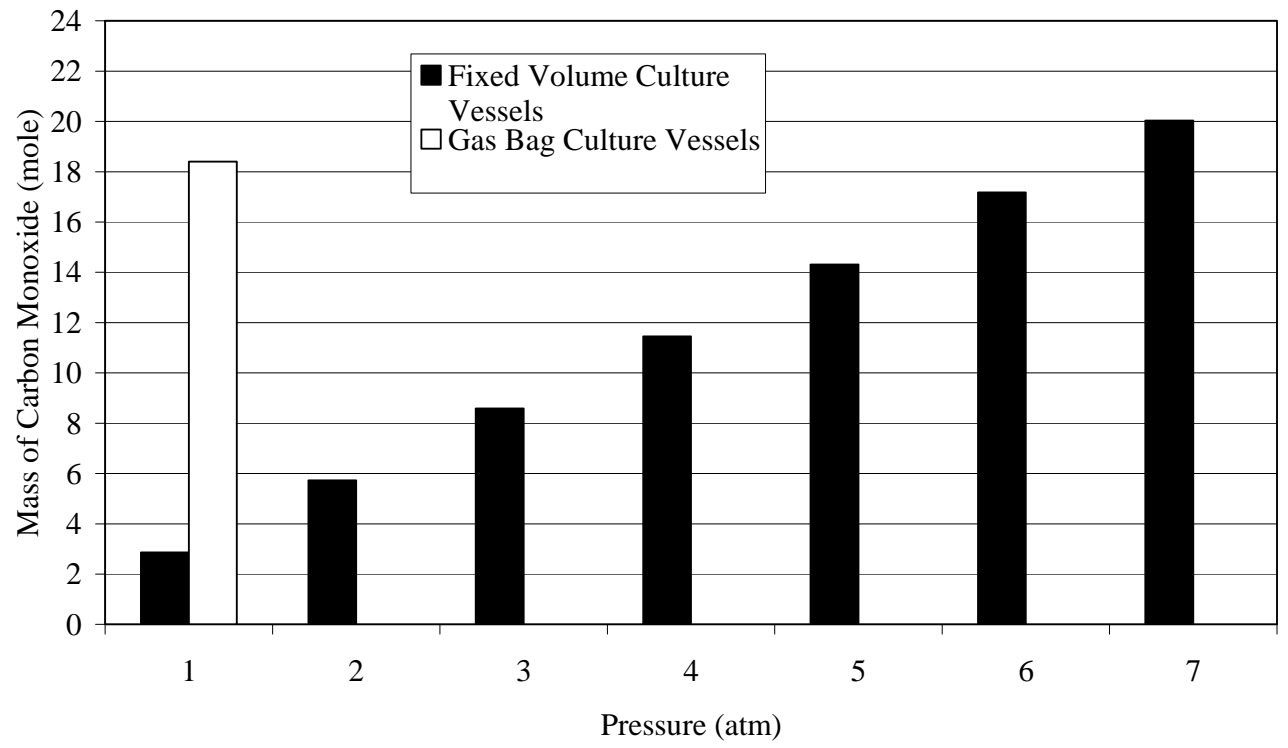

Figure 5. The effects of pressure on the mass of $\mathrm{CO}$ in fixed volume vessels compared to the mass of $\mathrm{CO}$ in a gas-sampling bag. Symbols: white bar, Gas Bag Culture Vessel; black bars, Fixed Volume Culture Vessels.

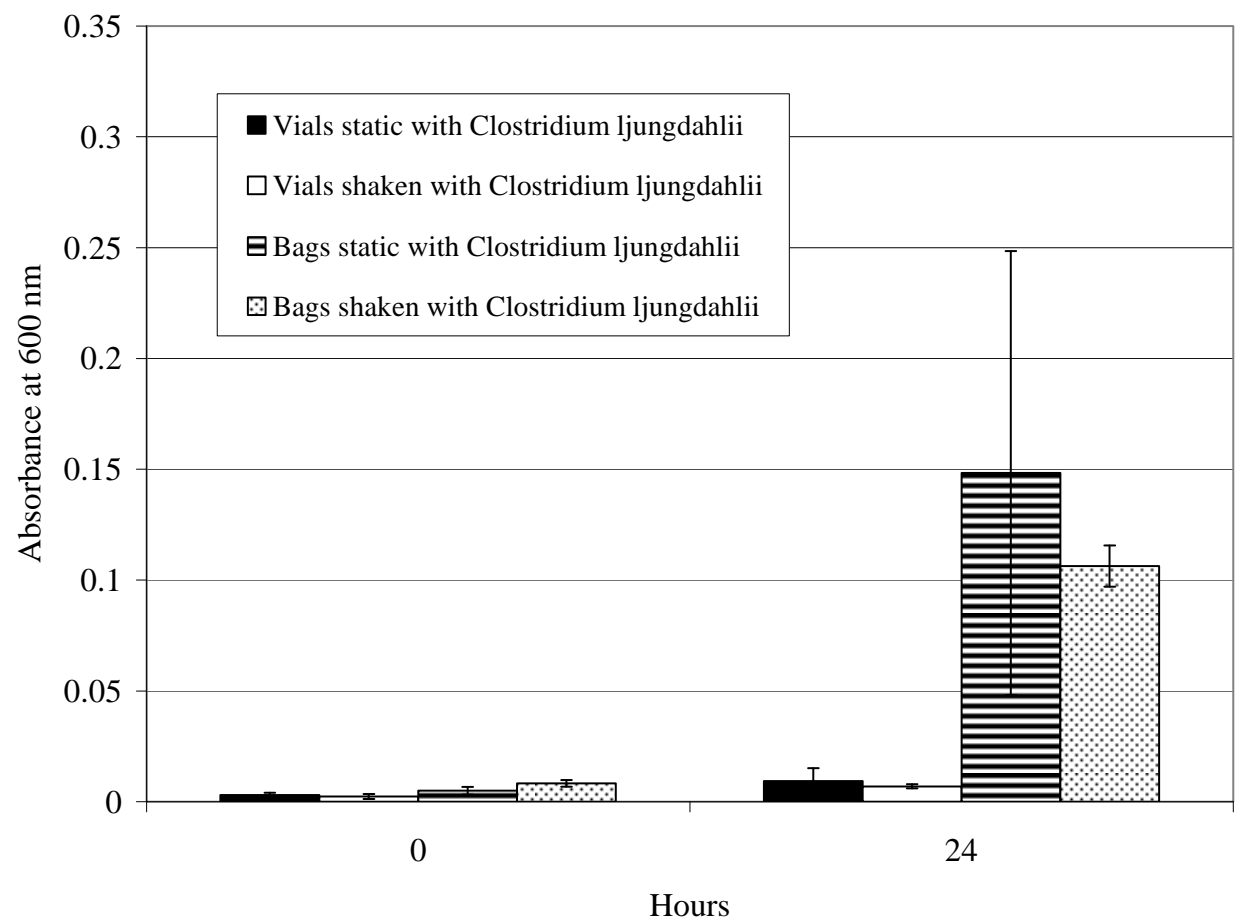

Figure 6. A comparison of vials and gas sampling bags for growing Clostridium ljungdahlii on $\mathrm{CO}$ as the sole carbon and energy source while incubated statically and shaken. 


\section{EVALUATION OF THE IMPACT OF NUTRIENT SOLUTION COMPOSITION AND NUTRIENT SOLUTION DOSING ON THE METHANOGENIC CONVERSION OF A SYNTHETIC ACETIC ACID SOLUTION \\ Performer: Mississippi State University}

\section{INTRODUCTION}

As the earth's natural resources continue to be depleted, the necessity to investigate alternative energy sources becomes increasingly more pressing. Each year the biosphere produces an estimated seven times more energy in the form of biomass than what is currently consumed. Ethanol produced from biomass represents an alternative fuel generally considered one of the most promising. One production technique currently under development is the production of ethanol from gasified waste products. Waste biomass sources are introduced into a gasifier where a synthesis gas consisting primarily of hydrogen, carbon monoxide, and carbon dioxide is produced. Anaerobic bacteria then convert these gas constituents into ethanol within a fermentor.

An issue affecting the potential for commercial development of this process is the management of the acetic acid that is formed as a fermentation byproduct. Approximately 1 gram of acetic acid is formed for every 5 grams of ethanol produced [6]. Currently, most designs propose treating the distillation bottoms as a process wastewater, as recovering the acetic acid is prohibitively expensive. However, discarding a wastewater with such a high organic content is expensive and a waste of valuable organic carbon that may be used for secondary production of another beneficial product. A more resourceful approach for managing the acetic acid in the waste stream involves utilizing methanogenic fermentation to produce biogas. Microorganisms known as methanogens consume acetic acid to produce methane and carbon dioxide under anaerobic conditions. The theoretical yield for this conversion is 1 mole of methane and 1 mole of carbon dioxide produced for every mole of acetic acid consumed [1]. Numerous species have been identified as highly capable of producing methane from acetic acid, and successful production of fuel-grade methane from the anaerobic degradation of other types of wastewaters has been well documented; however, the only two genera of methanogens known to utilize acetate as the sole energy source are Methanosarcina sp. and Methanosaeta sp. (formerly known as Methanothrix) [4]. However, there are several notable differences between this 
research and previous work. Conventional anaerobic fermentors typically treat a waste stream with a relatively low Chemical Oxygen Demand (less than 2000 mg/L) containing a mixture of volatile fatty acids [5]. This work evaluated treatment of wastewater having COD values of approximately 2500 and $13000 \mathrm{mg} / \mathrm{L}$ with acetic acid being the sole carbon source. Additionally, most applications of this process have primarily been concerned with reducing the COD of the wastewater to levels acceptable for discharge to the environment or publicly owned treatment works (POTW); optimizing methane production was of secondary concern [8]. This research focused on maximizing both the amount of biogas produced and increasing the methane content of the biogas with the understanding that the conversion would produce an outlet stream with a much lower COD value, suitable for discharge to a POTW.

It is well known that methanogens require a variety of nutrients and trace metals for optimum growth and methane production. Previous work analyzing 10 different strains of methanogens produced the following order of importance regarding the elemental composition: $\mathrm{Mg} \approx \mathrm{Ca}>\mathrm{Fe}>\mathrm{Zn} \geq \mathrm{Ni}>\mathrm{Co} \approx \mathrm{Mo}>\mathrm{Cu}>\mathrm{Mn}$ [9]. Nickel is especially important as a component of Factor $F_{430}$, a coenzyme found in all methanogens that is possibly involved in electron transfer. Other enzymes important to methanogenesis that also contain nickel are methyl reductase and carbon monoxide dehydrogenase. Cobalt and iron are required for the production of corrinoids (found in all methanogens) and cytochromes (found in the acid-utilizing methanogens). The specific function of other minerals such as molybdenum, calcium, zinc, and magnesium is unknown and could possibly differ with regard to genus and species [3]. Deficiencies in these metals can severely limit the productivity of an anaerobic digester; however, literature reports there are orders of magnitude differences between the optimum amounts of these metals that should be added [2].

Specifically, this work evaluated the impacts of the nutrient solution composition and nutrient solution dosing in relation to optimizing the methane yield. Since the source of methanogens for these experiments was anaerobic digester sludge, the primary goal was to determine if the sludge contained sufficient nutrients and trace metals to optimize methane production. In addition, the rate of methane production was 
evaluated and optimized with respect to the amount of nutrients added to the experiments.

\section{METHODS AND MATERIALS}

\section{Reactor Design}

All experiments were conducted in $500 \mathrm{~mL}$ round, flat bottom flasks custom made by Ace Glass (Vineland, NJ, USA). Each flask had three threaded necks, and the total volume of the flasks ranged from 505 to $560 \mathrm{~mL}$. The center neck of each flask was fitted with an adapter consisting of a 1.5-2.0 mm glass capillary tube and a threaded bushing with a $1.5 \mathrm{~mm}$ bored hole. The capillary tube and bushing adapter was attached to the reactor through threaded nylon bushing with a $7.5 \mathrm{~mm}$ center hole. The capillary tube portion of the adapter fit inside the hole of the bushing and was sealed with an o-ring. The bushing was also sealed to the reactor with an o-ring. An Agilent long-life septa was placed between the bushing and capillary tube to facilitate gas sampling.

One of the two larger side necks on the reactors was fitted with a solid threaded PTFE plug sealed with an o-ring. The second side neck was fitted with an Ashcroft test pressure gauge, used to monitor gas production and determine when gas and liquid sampling was necessary. Each Ashcroft pressure gauge (case type 1084) was 316 Stainless Steel and measured from 0-15 psig. Prior to conducting experiments, each of the reactors was pressure tested by injecting nitrogen into the reactor until the pressure gauge read approximately $10 \mathrm{psig}$. All of the fittings were checked for leaks, and pressurized reactors were incubated overnight to verify that the pressure could be maintained.

\section{Solution Preparation}

All of the acetic acid solutions were prepared using 97\% (by weight) glacial acetic acid (Fisher Scientific) and distilled water. The initial acetic acid concentration in all experiments was $2 \mathrm{~g} / \mathrm{L}$. Nutrients were added to the acetic acid solution as recommended by Speece [8]. The nutrient solution contained $\mathrm{NH}_{4} \mathrm{Cl}(12.608 \mathrm{~g} / \mathrm{L})$, $\mathrm{KH}_{2} \mathrm{PO}_{4}(0.429 \mathrm{~g} / \mathrm{L}), \mathrm{Na}_{2} \mathrm{~S}_{2} \mathrm{O}_{3} \cdot 5 \mathrm{H}_{2} \mathrm{O}(2.554 \mathrm{~g} / \mathrm{L}), \mathrm{CaCl}_{2} \cdot 2 \mathrm{H}_{2} \mathrm{O}(0.477 \mathrm{~g} / \mathrm{L}), \mathrm{MgCl}_{2} \cdot 6 \mathrm{H}_{2} \mathrm{O}$ $(0.151 \mathrm{~g} / \mathrm{L}), \mathrm{FeCl}_{2} \cdot 6 \mathrm{H}_{2} \mathrm{O}(0.0819 \mathrm{~g} / \mathrm{L}), \mathrm{NiCl}_{2} \cdot 6 \mathrm{H}_{2} \mathrm{O}(0.0162 \mathrm{~g} / \mathrm{L}), \mathrm{CoCl}_{2} \cdot 6 \mathrm{H}_{2} \mathrm{O}(0.0121$ 
$\mathrm{g} / \mathrm{L})$, and $\mathrm{ZnCl}_{2}(0.0417 \mathrm{~g} / \mathrm{L})$, and one $\mathrm{mL}$ of nutrient solution was added for every $\mathrm{g}$ of acetic acid per $\mathrm{L}$ of solution. The $\mathrm{pH}$ of the combined acetic acid and nutrient solution was controlled using a solution of approximately $20 \%$ sodium hydroxide. Once the acetic acid and nutrient solution was prepared, the initial $\mathrm{pH}$ of the solution was adjusted to 7 (using the sodium hydroxide solution). All of the chemicals used to prepare the solutions were purchased from Fisher Scientific.

\section{Experiment Preparation}

To maintain anaerobic conditions in the reactor, all experiments were prepared in an anaerobic flexible vinyl chamber (Coy Products, Grass Lake, MI, USA). The vinyl chamber atmosphere contained a $95 \%$ nitrogen-5\% hydrogen gas mixture. To ensure that the solutions were anaerobic, the acetic acid nutrient solution was placed in the reactor in an open container and allowed to sit in the chamber for several hours. The open reactors were also placed in the chamber along with the necessary amount of digested sewage sludge required to inoculate the reactors. The digested sewage sludge was obtained from the Tuscaloosa, AL and the Vicksburg, MS sewage treatment plants. After equilibrating in the chamber, $20 \mathrm{~mL}$ of digester sludge and $250 \mathrm{~mL}$ of acetic acid nutrient solution were added to the reactors. The reactors were then sealed, removed from the anaerobic chamber, the pressure gauges were aligned to zero, and the reactors were incubated at $35^{\circ}$.

\section{Reactor Operation}

The reactors were incubated at $35^{\circ} \mathrm{C}$ for the entire length of each experiment. The pressure in the reactors was monitored, and experiments were deemed complete when the reactor pressure no longer increased, indicating cessation of gas production. Each time the pressure in a reactor reached 10-15 psig, gas samples were collected using a $250 \mu \mathrm{L}$ gas-tight Luer Lok® syringe fitted with a 23 gauge needle. A $115 \mathrm{~mm}$ long needle was inserted through the bored hole in the bushing in the center sample port of the reactors. The needle length ensured that samples were collected from the headspace of the reactor and not from the capillary tube. After analyzing the gas, reactors were opened in the anaerobic chamber to release the pressure and collect 
liquid samples for $\mathrm{pH}$, redox potential, chemical oxygen demand (COD), and high pressure liquid chromatography (HPLC) analysis.

\section{Sample Analysis}

Gas and liquid samples from the reactors were analyzed for several different parameters. Gas samples were analyzed for methane and carbon dioxide using an Agilent $6890 \mathrm{~N}$ gas chromatograph (GC) with a thermal conductivity detector (TCD). A volume of $100 \mu \mathrm{L}$ of gas was injected into the GC. The column used to separate the methane and carbon dioxide was a Supelco 80/100 Porapak column. The column was 6 ' long by 1/8" ID and made of Stainless Steel. The standard curves for methane and carbon dioxide were prepared by injecting fixed amounts $(10,50$, and $100 \mu \mathrm{L})$ of pure gas and plotting the resulting response. The operating parameters of the GC were as follows: initial oven temperature of $100^{\circ} \mathrm{C}$ for 3 minutes, ramped to $150^{\circ} \mathrm{C}$ at $25^{\circ} \mathrm{C}$ per minute; front inlet temperature of $200^{\circ} \mathrm{C}$; front detector temperature of $250^{\circ} \mathrm{C}$; heater temperature of $250^{\circ} \mathrm{C}$; reference flow rate of $20 \mathrm{~mL}$ per minute; helium make-up flow rate of $5 \mathrm{~mL}$ per minute; column flow rate of $20 \mathrm{~mL}$ per minute; and total flow rate of $20.8 \mathrm{~mL}$ per minute.

Liquid samples were analyzed for Chemical Oxygen Demand (COD), acetic acid concentration, $\mathrm{pH}$, and oxidation/reduction potential (to verify that the reactor systems were anaerobic). COD analysis was completed according to Hach Method 8000 for the $0-15,000 \mathrm{mg} / \mathrm{L}$ range. A volume of $200 \mu \mathrm{L}$ of liquid sample was added to COD High Range Plus Digestion Reagent Vials, and the vials were incubated in a Hach COD Reactor at $150^{\circ} \mathrm{C}$ for 2 hours. Once the samples were completely digested and cooled, the COD concentration in $\mathrm{mg} / \mathrm{L}$ was measured using a Hach $\mathrm{DR} / 2010$ Spectrophotometer. Every time a reactor was opened and sampled, enough liquid was collected to perform the COD analysis in duplicate.

The acetic acid concentration in the liquid samples was measured using a Waters HPLC. Before sample analysis, each $1 \mathrm{~mL}$ volume of liquid sample was acidified with 4-5 drops of HPLC grade $85 \%$ o-phosphoric acid. Liquid samples that were not immediately analyzed were stored in the freezer at $0^{\circ} \mathrm{C}$. Just prior to analysis, liquid samples were filtered using a $1.0 \mathrm{~mL}$ Luer Lok® syringe and a $25 \mathrm{~mm}$ syringe 
filter containing 0.2-0.6 $\mu \mathrm{m}$ pore size filter paper. The HPLC was fitted with a $4 \times 23 \mathrm{~mm}$ guard column followed by a Waters YMC ODS-AQ column specific for carboxylic acids. The operating parameters of the HPLC were as follows: Mobile phase of $20 \mathrm{mM} \mathrm{PO}_{4}$ at $\mathrm{pH} 3.5$; temperature of $30^{\circ} \mathrm{C}$; flow rate of $0.7 \mathrm{~mL} /$ minute; injection volume of $10 \mu$; and a $220 \mathrm{~nm}$ UV and 0.08 AUFS detector/sensor.

An Accumet AP62 portable $\mathrm{pH} / \mathrm{mV}$ meter fitted with the appropriate probes was used to collect $\mathrm{pH}$ and oxidation/reduction potential data. The $\mathrm{pH}$ was determined using an Accu-TupH rugged bulb combination electrode, and the oxidation/reduction potential was determined using an Accumet platinum/ $\mathrm{Ag} / \mathrm{AgCl}$ combination electrode.

Data was statistically evaluated using a t-test to compare two means at $90 \%$ and 95\% confidence intervals.

\section{RESULTS}

\section{Nutrient Solution Composition}

Experiments were conducted to evaluate the effect of the composition of the nutrient solution on acetic acid degradation by a mixed culture of methanogens obtained from a municipal anaerobic digester. These experiments were conducted with both 2 $\mathrm{g} / \mathrm{L}$ and $8 \mathrm{~g} / \mathrm{L}$ acetic acid solution adjusted to an initial $\mathrm{pH}$ of 7.0. The four nutrient solution compositions evaluated were 1) the addition of no additional nutrients, 2) the addition of nitrogen and phosphorus only; 3) the addition of nitrogen, phosphorus, cobalt, and iron only; and 4) the addition of the complete nutrient solution, containing all of the previous ingredients plus sulfur, calcium, magnesium, nickel, and zinc. The amount of each component added was based on recommendations by Speece [8]. Due to equipment constraints, one experiment for each of the four conditions condition was conducted, and the 4 experimental conditions were evaluated over two independent repetitions.

The effect of the composition of the nutrient solution on the degradation of acetic acid is illustrated by comparing the estimated experimental yield for each of the experiments. Figure 1 shows the ratio of moles of methane and total gas produced (methane and carbon dioxide) per mole of acid consumed in these experiments. As the nutrient additions increased from none to the complete solution, the methane yield 
increased from 0.8 to about 0.96 moles produced per mole of acid consumed. In calculating the total yield, it was assumed that all of the acetic acid was consumed. This approximation was deemed valid because the final COD of the liquid was less than $1000 \mathrm{mg} / \mathrm{L}$, a similar COD value for a control experiment that contained biomass but no acetic acid. Approximately $97 \%$ of the total yield was due to the methane produced, while only $3 \%$ was from the carbon dioxide. The amount of methane produced increased by over $22 \%$ once nitrogen and phosphorus and trace metals were added.

The COD reduction (accuracy of $\pm 100 \mathrm{mg} / \mathrm{L}$ ) was also affected by the nutrients present in the solution, as shown in Figure 2. The average initial COD for the $2 \mathrm{~g} / \mathrm{L}$ experiments was $2910 \mathrm{mg} / \mathrm{L}$. At the conclusion of the experiment days, the average COD values of the solution containing no additional nutrients and nitrogen and phosphorus only were 1050 and $1025 \mathrm{mg} / \mathrm{L}$, respectively. The experiments containing trace metals in addition to nitrogen and phosphorus had average COD values of 545 and $670 \mathrm{mg} / \mathrm{L}$, respectively. The final COD of the experiments with $8 \mathrm{~g} / \mathrm{L}$ acetic acid improved from an average of $1180 \mathrm{mg} / \mathrm{L}$ with no added nutrients to an average of 725 $\mathrm{mg} / \mathrm{L}$ with the addition of the complete nutrient solution. Experiments containing no nutrients and or trace metals were able to degrade the acetic acid likely because microand macronutrients were present in the digested sludge used as the source of biomass. However, the addition of nitrogen and phosphorus as well as trace metals increased the capability of the system to degrade acetic acid and improved the methane and total biogas yield.

\section{Nutrient Solution Amounts}

The next series of experiments were designed to evaluate the impact of increasing the amount of the complete nutrient solution added to the experiments. Initially, experiments were conducted using $8 \mathrm{~g} / \mathrm{L}$ acetic acid solution that contained 1 , 2 , and 4 times the recommended amount of nutrients and trace metals as suggested by Speece [8]. The higher acid concentration was targeted for these experiments due to the extended time (average of 67 days) required to complete the methanogenic conversion of an $8 \mathrm{~g} / \mathrm{L}$ acid solution. Due to the excessive length required to complete the experiments, only the 4-fold increase in nutrients was tested multiple times, and the data reflects results from 11 experiments. Experiments were conducted in duplicate; 
however, some experiments were not completed due to gas leaks from the reactor (as reflected in the odd number of data points).

The addition of 2 times and 4 times the recommended amount of nutrients most notably affected the gas production rate and time required to complete the experiments. Figure 3 compares the COD reduction for each (1X,2X, and $4 X)$ of the nutrient doses. The additional nutrients reduced the time to complete the experiments by over half, from 95 days to 42 days. Ultimately, the final COD values for each of the experiments were comparable at $725(1 \mathrm{X}), 650(2 \mathrm{X})$, and $675(4 \mathrm{X})$; however, the time required to achieve the COD reduction decreased significantly as nutrient dosing increased.

To further evaluate the impact of nutrient dosing, the amount of nutrient solution added was increased to 10 times the recommended amount. The 10-fold increase in nutrients was also evaluated using duplicate experiments; however, not all of the experiments proceeded to completion resulting in the collection of 13 data points. Figure 4 shows how the four different dosing schemes compared in terms of the increase in reactor pressure per day. Averaging the results of multiple experiment replications showed that increasing the nutrients ten-fold does not improve reactor performance over the four-fold increase, but actually may somewhat impede reactor performance. Further comparison of the estimated gas yields from the 2, 4, and 10 times increase in nutrients in Figure 5 shows that the 10-fold increase reduces the methane and total gas yields as compared to those obtained with the 2-fold and 4-fold increase in nutrients. The average results from the 4 -fold increase in nutrients do show a small advantage over the 2 -fold increase of about 0.09 psig per day.

In addition to COD, the liquid from these experiments was also analyzed using HPLC to determine the actual acetic acid concentration and this data is shown in Figures $6 \mathrm{a}$ and $6 \mathrm{~b}$. COD reduction was comparable for the 4-fold and 10-fold doses of nutrients and trace metals, with final values average $826 \mathrm{mg} / \mathrm{L}$ and $850 \mathrm{mg} / \mathrm{L}$, respectively. The HPLC data verified the results from COD analysis, as the average acid concentration at the end of the experiments had decreased to $0.1925 \mathrm{~g} / \mathrm{L}$ for the $4 \mathrm{X}$ experiments and $0.165 \mathrm{~g} / \mathrm{L}$ for the $10 X$ experiments. This data shows that significant acetic acid degradation was achieved in the systems. It also shows that the 10 -fold increase in nutrients decreased the time required to degrade the acid by an average of 
13 days as compared to the experiments with the 4-fold increase; however, the final COD and acetic acid concentrations were the same.

\section{DISCUSSION}

\section{Nutrient Solution Composition}

It is well documented that pure and enriched cultures of methanogens require specific amounts of nitrogen, phosphorus, iron, cobalt, and other trace metals $[3,8]$. The purpose in varying the nutrient solution compositions was to determine if the secondary anaerobic digester sludge used as the source of methanogens for these experiments contained sufficient quantities of nutrients and trace metals to sustain methane production. The increase in methane yield observed upon the addition of nutrients and trace metals as compared to the yield produced with no additional nutrients or trace metals added is significant at the $90 \%$ confidence interval but not at the $95 \%$ confidence interval. Differences in the biological systems from experiment to experiment resulted in large values for standard deviation. However, evaluation of both the methane yield data and the COD data supports the conclusion that the addition of additional nutrients and trace metals improves system performance. On average, the addition of additional nitrogen, phosphorus, and trace metals increased methane production by about $22 \%$. As shown by the results, secondary digester sludge alone does not contain enough nutrients and trace metals to sustain maximum gas production under these experimental conditions. However, sufficient amounts of nutrients and trace metals did remain in the source inoculum to support methanogenesis

Literature also reports that the theoretical gas yield for methanogenesis should be one mole of carbon dioxide and one mole of methane produced for every mole of acetic acid consumed [1]. These experiments produced the expected yield of methane but not carbon dioxide. The reduced carbon dioxide yield was likely a result of the high $\mathrm{pH}$, which increased from 7 to about 8 during the length of the experiments. At higher $\mathrm{pH}$ values, carbon dioxide will form carbonates that dissolve in water [7]. GC analysis of the gas did not detect the carbon dioxide dissolved in solution, and as a result, the amount of carbon dioxide produced and the total gas yield were lower than predicted by stoichiometry. 


\section{Nutrient Solution Amounts}

It can be seen from the data that the addition of 2 times, 4 times, and 10 times the recommended amount of nutrients and trace metals improves reactor performance in terms of reducing the length of time required to complete experiments with a high initial acetic acid concentration. While the final COD values are comparable, the amount of time required to achieve this COD reduction can be reduced by as much as half by quadrupling the amount of nutrients and trace metals present. Further comparison of the data reveals that, while the four-fold increase produced the highest yield, there is no significant difference when compared to the yield resulting from a twofold increase in nutrients and trace metals. The only noticeable advantage of a two to four-fold increase is increase in the gas production.

Increasing the total nutrient addition by ten-fold may be detrimental to biogas production, as shown by the decrease in yield. The decrease in gas production rate and methane yield resulting from the ten-fold increase in nutrients was not unexpected, as earlier research has shown that, if present in high enough concentrations, potassium and ammonium can inhibit the conversion of acetic acid to methane [10].

The results from these experiments suggest that doubling or quadrupling the nutrient and trace metal additions could help optimize the production of biogas when the source inoculum is a mixed culture obtained from an existing anaerobic digester. The increased gas production rates would minimize the hydraulic residence time required to degrade higher concentrations of acetic acid, such as those expected in the wastewater resulting from the production of ethanol from synthesis gas. The costs of additional nutrient and trace metal additions would need to be compared with the operating costs associated with longer reactor operation in order to determine which of the two options (doubling or quadrupling) is most cost effective. 


\section{REFERENCES}

1. Ferry, James G (1992) Methane from acetate. J Bacteriol: 174.17: 5489-5495.

2. Gonzalez-Gil G, Kleerebezem R, Lettinga G (1999) Effects of nickel and cobalt on kinetics of methanol conversion by methanogenic sludge as assessed by on-line $\mathrm{ch}_{4}$ monitoring. Appl Environ Microb: 65.4: 1789-1793.

3. Jarrell K, Kalmokoff M (1988) Nutritional requirements of the methanogenic archaebacteria. Can J Microbiol: 34: 557-576.

4. Jetten M, Alfons S, Stams J, Zehnder A (1992) methanogenesis from acetate: a comparison of the acetate metabolism in methanothrix soehngenii and methanosarcina spp. FEMS Microbiol Rev: 88: 181-198.

5. Kato M, Rebac S, Lettinga G (1999) Anaerobic treatment of low-strength brewery wastewater in expanded granular sludge bed reactor. Appl Biochem and Biotech: 76: $15-32$.

6. Klasson K, Ackerson M, Clausen E, Gaddy J (1991) Fuel: 70.5.

7. Snoeyink V, Jenkins D (1980) Water Chemistry. New York: John Wiley and Sons, Inc.

8. Speece R (1996) Anaerobic Biotechnology for Industrial Wastewaters. Nashville, TN: Archae Press.

9. Takashima M, Speece R (1990) Mineral Requirements for Methane Fermentation. Crit Rev Env Contr: 19.5: 465-479.

10. Van den Berg L, Patel G, Clark D, Lentz C (1976) Factors affecting rate of methane formation from acetic acid by enriched methanogenic cultures. Can J Microbiol:

22: $1312-1319$. 


\section{FIGURES}

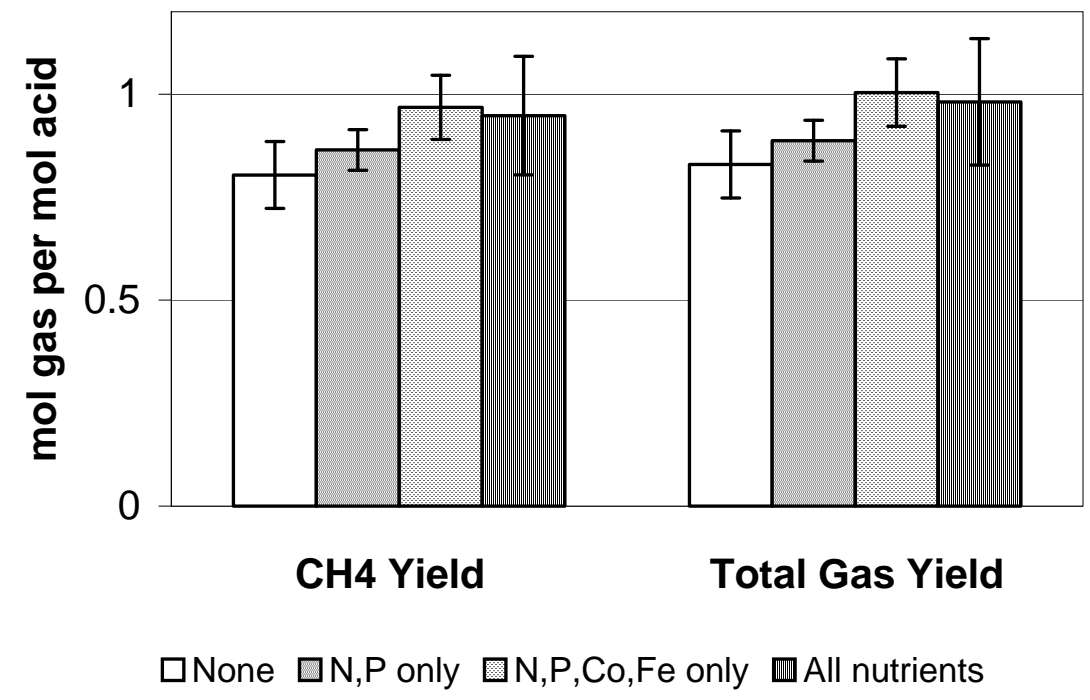

Figure 1: Experimental Yield, Various Nutrient Solution Compositions

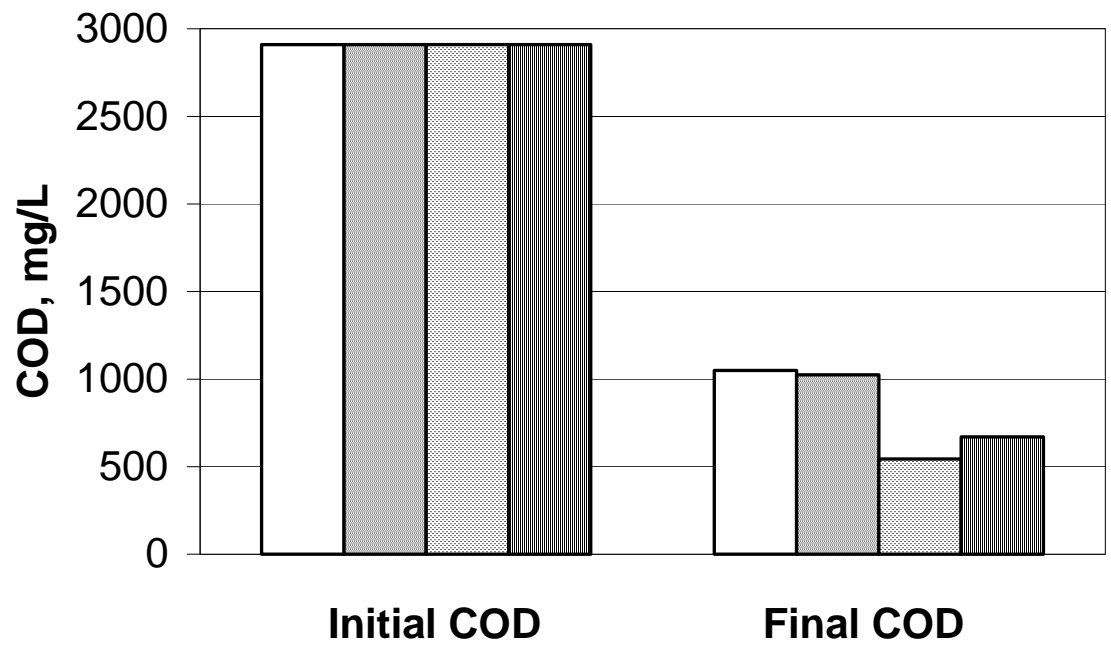

$\square$ None $\quad \square$ N,P only $\quad \square N, P, C o, F e$ only $\quad$ m All Nutrients

Figure 2: COD Reduction, 2 g/L Acetic Acid, Various Nutrient Solution Compositions 


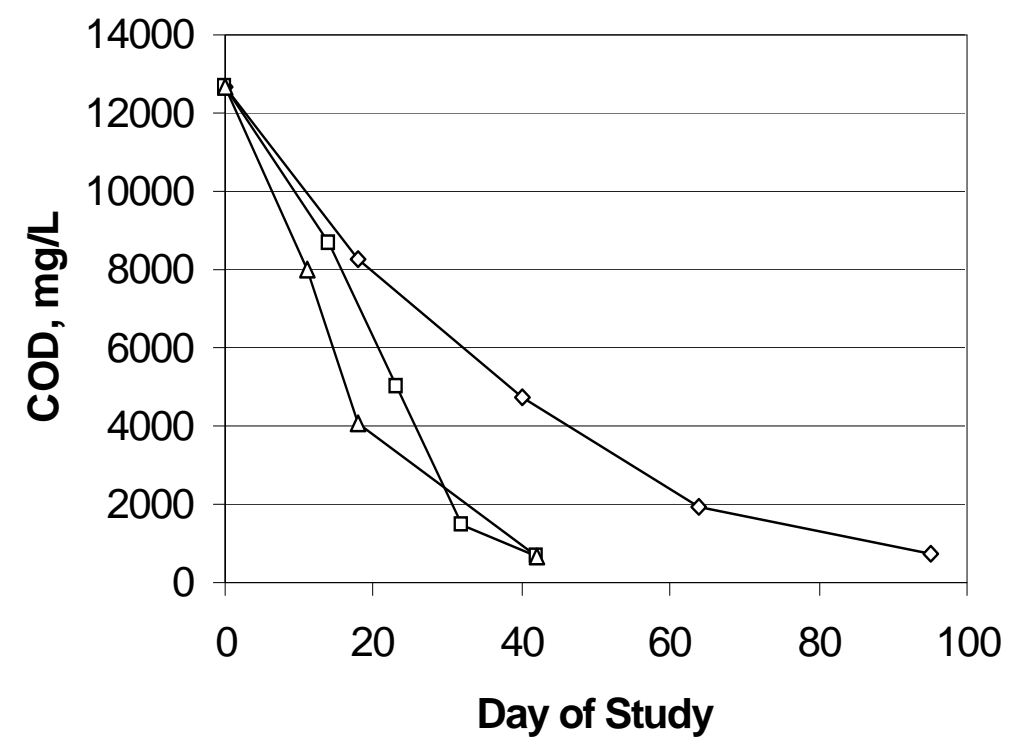

$\diamond-1 X$ all nutrients $\rightarrow-2 X$ all nutrients $\rightarrow-4 X$ all nutrients

Figure 3: COD Reduction, 8 g/L Acetic Acid, Various Nutrient Doses

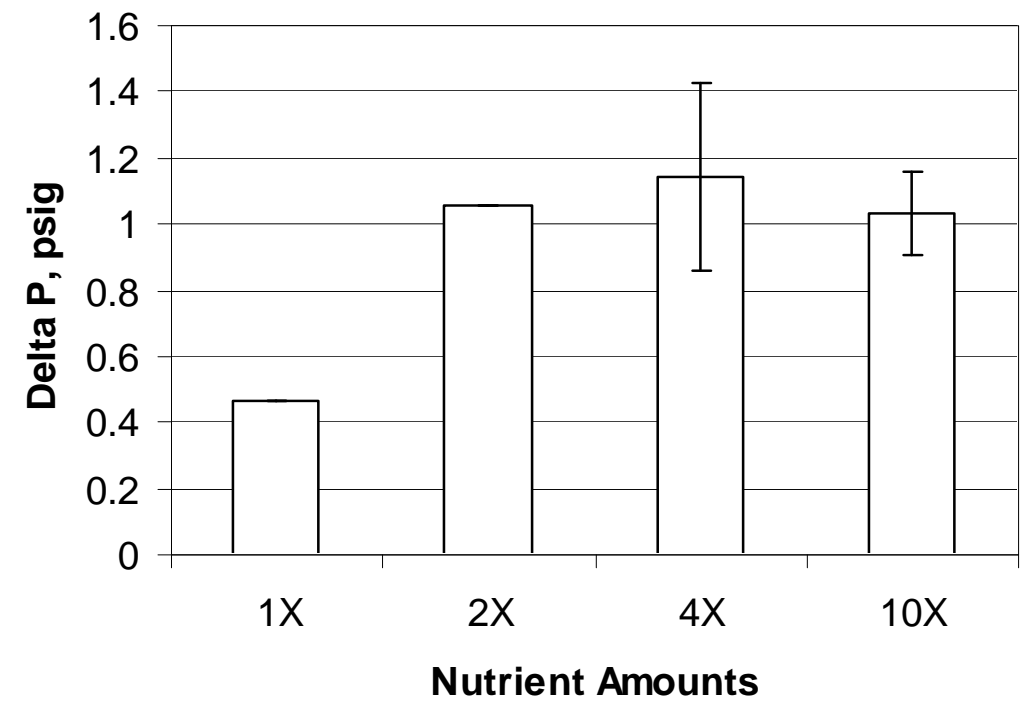

Figure 4: Average Reactor Pressure Increase per Day, Various Nutrient Doses 


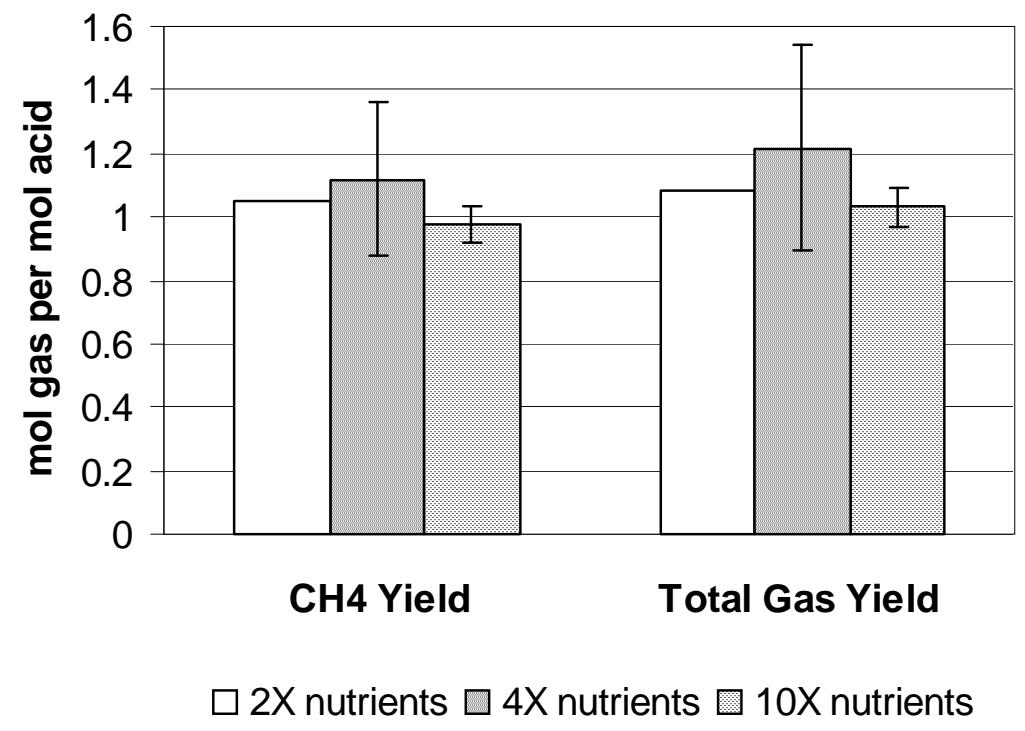

Figure 5: Experimental Yield, 8 g/L Acetic Acid, Various Nutrient Doses 


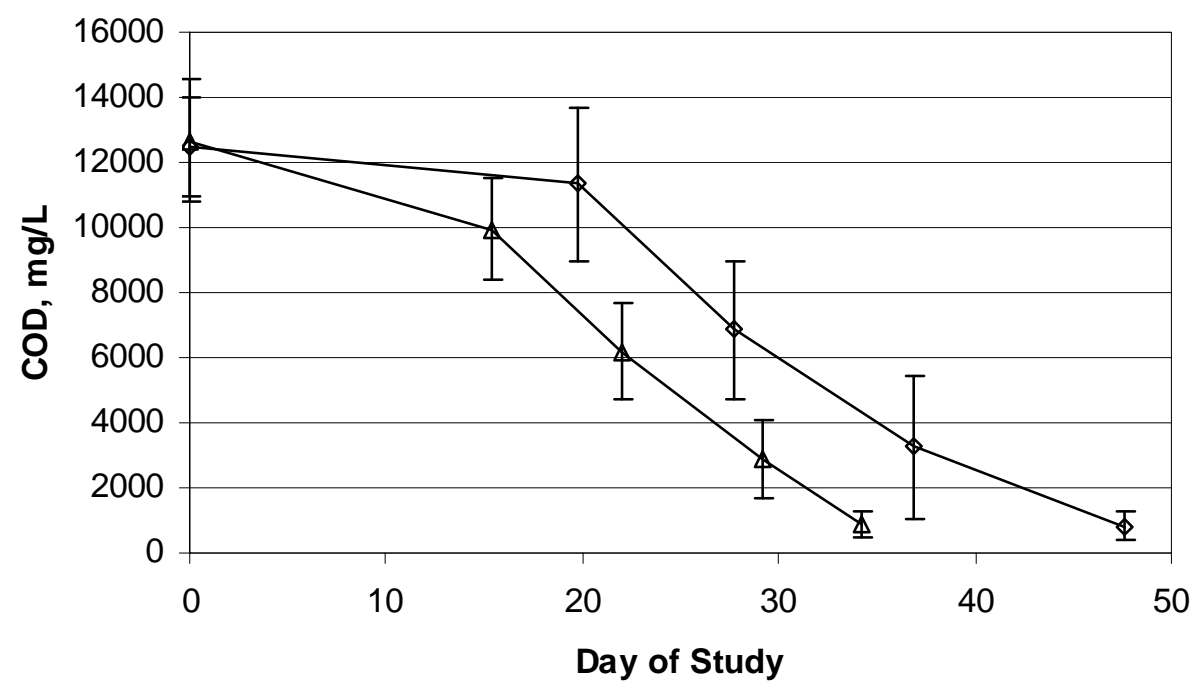

$\multimap 4 X \multimap 10 X$

Figure 6a: COD Reduction, $8 \mathrm{~g} / \mathrm{L}$ Acetic Acid, 4X and 10X Recommended Nutrients/Trace Metals

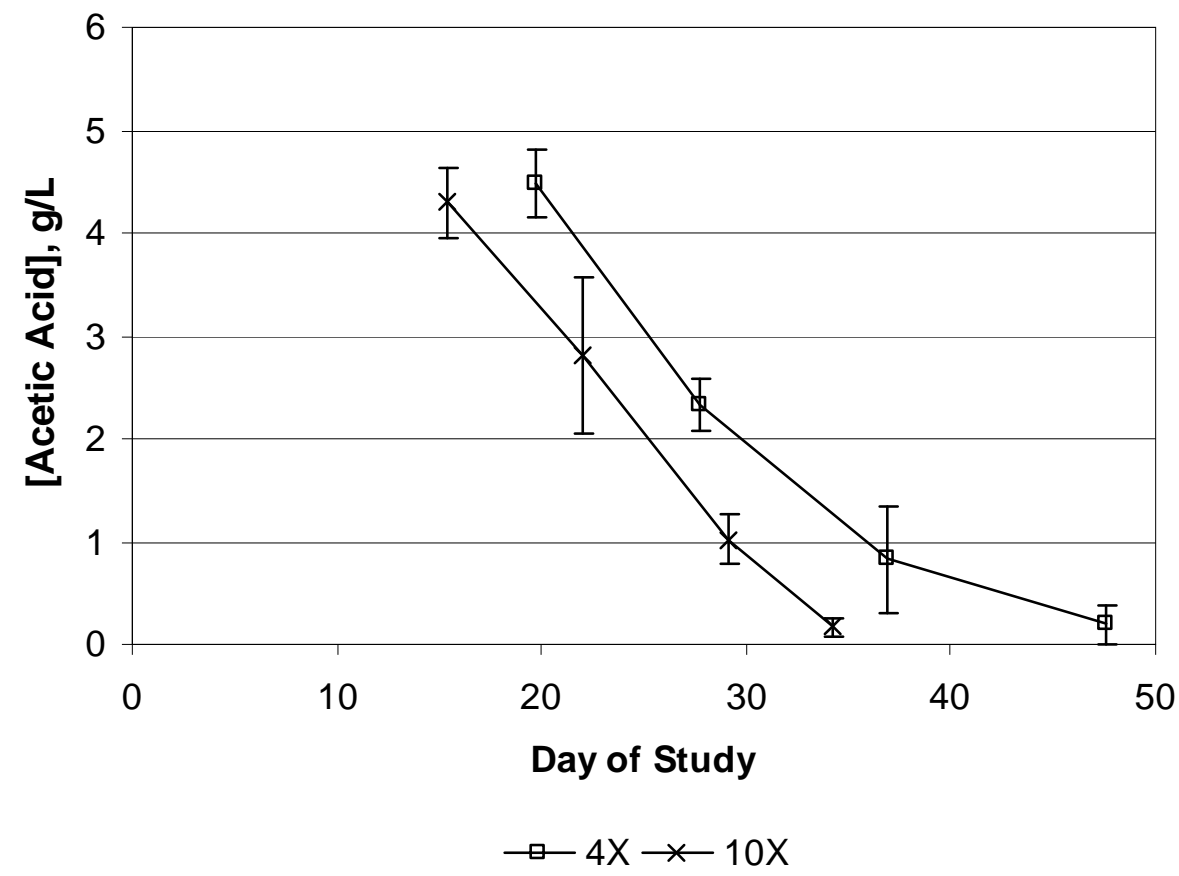

Figure 6b: Acetic Acid Degradation, $8 \mathrm{~g} / \mathrm{L}$ Acetic Acid, 4X and 10X Recommended Nutrients/Trace Metals 


\section{EVALUATION OF THE USE OF METHANOGENESIS TO CONVERT THE WASTE ACETIC ACID TO METHANE Performer: University of Mississippi}

\section{PROBLEM STATEMENT}

Production of ethanol is accompanied by formation of acetic acid in the distillate bottoms as a by-product. Currently these bottoms are treated as process water. Treating an acetic acid laden wastewater (approximately $5000 \mathrm{mg} / \mathrm{L}$ ) containing this high BOD is an expensive option and loss of valuable carbon source.

\section{BACKGROUND}

Major car companies are manufacturing flexible fuel vehicles (FFV) that work on E85 gas (85\% ethanol and 15\% gasoline). In contrast to reformulated gasoline that is used presently, ethanol helps maintain clean air, has low toxicity, is highly miscible in water and is easily biodegradable. Biomass fermentation to produce ethanol, major component of E85 [18] is exhibiting the potential to be an extremely environment friendly and economical option. Studies indicate that fermentations optimized for ethanol production from biomass result in significant acetic acid concentrations in the distillate bottoms. Currently, most facilities plan to treat the bottoms as a process wastewater stream. Treating an acetic acid laden wastewater $(5 \mathrm{gm} / \mathrm{L}$ of acetic acid per $25 \mathrm{gm} / \mathrm{L}$ of ethanol produced) with such high BOD is an expensive option. Treatment also eliminates a valuable carbon source that can be used for the production of a potentially beneficial product. A better option uses anaerobic digestion of the acetic acid laden wastewaters to produce methane (methanogenesis). Methane can then be used as a fuel within the facility.

In methanogenesis a large number of Euryarchaeota produce methane as an integral part of their catabolism. Most methanogens characterized to date utilize hydrogen and carbon dioxide but only two genera, Methanosarcina and Methanothrix (also called as Methanosaeta) [13] have been reported to produce methane from acetate. Methanosarcina utilizes other substrates such as hydrogen and carbon dioxide, methanol and methylamines; whereas Methanosaeta bacteria use only acetate as substrates. Production of methane from acetic acid by pure cultures is described as acetotrophic [2]. Three major physiological groups produce acetate - fermentors, 
syntrophs and acetogens. Though much work has been done on methanogenesis, little research has compared the inhibition of acetoclastic methanogens in a consortium due to competition with other bacteria. Most of the work on acetate in oxygen-limited environment is conducted with isolates and there are few available process details (components of the media and residence time) or nutritional studies involving mixed consortia, which impede design efforts.

It has been proven that ammonia, nitrates, and the reduced forms of nitrates, inhibit methanogenesis, but there is some doubt about the effect of ammonium salts. Some papers cite a positive effect [7]. Inhibition was observed especially for carbon dioxide utilizing bacteria [8]. At low concentrations $\mathrm{NH}_{4}{ }^{+}$ions seemed to increase the production of methane, while at higher concentrations, they inhibited the process [16]. The salt concentrations that mark the change over from enhancement to inhibition also vary depending on the type of ecosystem. This may result from a conversion of the higher concentration salts to ammonia [2]. There has been a lack of a systematic study involving ammonium chloride salts to effectively analyze its effect on mixed consortia.

Methane formation depends on sodium and is accompanied by the generation of a secondary sodium ion gradient [15] [14]. An analysis with different sodium acetate (50 and $250 \mathrm{mM}$ ) concentrations would help in correlating the carbon content and ammonium chloride required. It would be very beneficial to obtain a trend of methane production with respect to the concentration of salt added and explain the behavior. We utilized $0,10,20,56,100$ and $150 \mathrm{mM}$ concentrations of ammonium chloride to analyze their effect on methane production. Carbon dioxide production and nitrogen (headspace) consumption were also ascertained to reveal the reaction mechanism of the process. Secondary transfers were done to see the effect on residence time, carbon dioxide production and nitrogen consumption. Statistical analysis and modeling were also performed. 


\section{SUMMARY}

The potential production of methane from a methanogenic bioreactor was evaluated for its ability to convert the acetic acid within distillation bottoms into methane, which is recovered as an energy source. A secondary aspect of this project was to determine acetate treatment efficiencies for treatment of wastewaters containing high organic contents. The main objectives behind carrying out these experiments were to evaluate key design parameters such as cell resident time, volumetric methane production, and nutrient feed composition.

\section{APPROACH}

The initial sets of experiments were conducted in 150 or $30 \mathrm{ml}$ serum bottles sealed with rubber stoppers and aluminum crimps. Syringes $(3 \mathrm{ml})$ were utilized for inoculation. The solid inoculum was diluted with DI water to a slurry.

Subsequent gas production was evaluated after incubation $\left(32^{\circ} \mathrm{C}\right.$ and $\left.45^{\circ} \mathrm{C}\right)$, to study the effect of temperature on methanogenesis. Samples for analysis were then withdrawn when the pressure of the gas produced pushed up the plunger of the syringe. Inoculates were obtained from various sources (e.g. creek sediments, horse, deer and cow dung, rumen, etc). These sources were screened, based on the residence time and methane produced to select the most effective methanogenic source. The miscellaneous inoculate source in Table 1 stands for the sediments which were obtained from various parts of Oxford (MS 38655), including the waste water treatment plant.

The batch incubators were then scaled-up to custom-built $500 \mathrm{~mL}$, round, flatbottom flasks (Ace Glass Inc., Vineland, New Jersey). The flasks had a screw type fitting on top to mount a pressure gauge. At the side was another opening with a valve and septum arrangement shown in Figure 1 to allow gas sample removal frequently without allowing exposure to atmospheric oxygen.

As soon as positive pressure developed, gas samples were withdrawn at regular intervals (every 2-3 days) using $5 \mathrm{ml}$ gas tight syringes. Gas composition was analyzed in a Carle Gas Chromatograph (Model: 2158) with a Thermocouple Detector (TCD), coupled with a Hewlett Packard Integrator (Model: 3396C). 
Each $500 \mathrm{~mL}$ reactor, containing $250 \mathrm{~mL}$ media, was then sterilized by autoclaving at $120^{\circ} \mathrm{C}$ for 45 minutes. Each liter of media consisted $1 \mathrm{~mL}$ Cohen-Bazire's modified Hunters stock solution. Then $272.2 \mathrm{mg} / \mathrm{L}$ of $\mathrm{KH}_{2} \mathrm{PO}_{4}$ was added to the media. The estimated acetic acid concentration in the distillation bottoms, following fermentation of synthesis gas is $5 \mathrm{~g} / \mathrm{L}$ [4] [9]. In our work, we used sodium acetate at $6.805 \mathrm{~g} / \mathrm{L}(50 \mathrm{mM}, \mathrm{Ac} 1 \mathrm{x})$ and $34.025 \mathrm{~g} / \mathrm{L}(250 \mathrm{mM}, \mathrm{Ac5x})$ as the feed in place of acetic acid.

The valves were closed as soon as the reactors were removed from the autoclave. The sterilized media was allowed to cool to ambient conditions for one day. Inoculate, containing cow dung and cow rumen was diluted to give a slurry and then 50 $\mathrm{ml}$ of this slurry was added to each reactor. This ensured that the cell mass and the carbon content in the inoculum was distributed equally to all reactors. Considering the future scale-up of this project to an industrial level where it is not feasible to maintain strict anaerobic condition, strict anaerobic techniques were not followed during the inoculation. Rather oxygen contamination was minimized. The media was inoculated under nitrogen flushing. The initial gas analysis indicated the residual oxygen in the reactors to be from 3 to $5 \%$ molar. The oxygen level fell below $1 \%$ within 4 to 5 days. The reactors had a valve septum arrangement to facilitate gas sample removal at regular intervals with a gas tight syringe. Pressure gauges $\left(200\right.$ in $\left.\mathrm{H}_{2} \mathrm{O}\right)$ were mounted on each reactor to monitor the rate of production of gas inside the reactor. The reactors were incubated at $32^{\circ} \mathrm{C}$ in Precision economy static incubators from Fisher Scientific (Model: 13-255-7).

Secondary transfer experiments were also carried out using bacteria from the primary run with addition of nutrients. The procedure for media preparation for the secondary transfer runs was similar to that of the primary runs. The same experimental matrix was followed. Transfers were accomplished after allowing the primary incubation to continue for 75 days. At this point, samples for $\mathrm{pH}$ determination and fluorescence microscopy were collected. Inoculate was collected from the bottom of all the reactors and mixed. An oxygen limited environment was created by covering a styrofoam box (2' $\left.x 3^{\prime}\right)$ with a glove bag. Inoculate was distributed under nitrogen purging to ensure an oxygen limited environment. 


\section{OBSERVATIONS AND RESULTS}

Data obtained from the primary run shows that the yield was more than $100 \%$. This can be explained on the basis that two types of methanogens, namely the acetate utilizing and the hydrogen utilizing methanogens, produced methane simultaneously. The acetate utilizing methanogens give $1 \mathrm{~mol}$ of $\mathrm{CO}_{2}$ for every $1 \mathrm{~mol}$ of methane produced, while the hydrogen utilizing methanogens use $\mathrm{CO}_{2}$ along with hydrogen to give methane. Moreover, the lag time in the primary run was less for the low feed concentration as compared to the high feed concentration. Results of the secondary transfer run show almost a $70 \%$ reduction in lag time as compared to the primary runs. Also, there is difference between the trends of $\mathrm{CO}_{2}$ production. In the primary run the trend of $\mathrm{CO}_{2}$ production is exponential but in the transfer run it is not. The trend of $\mathrm{CO}_{2}$ production in the transfer run has negative slopes in indicating utilization of $\mathrm{CO}_{2}$ to produce methane. These results suggest that there is interaction between two different species of methanogens for production of methane, which ultimately leads to high yields of methane. Also methanogens were able to fix atmospheric nitrogen along with the ammonium chloride that was supplied as nutrient. The observation that ammonium chloride played an important role led to a more detailed study.

Production of methane from acetic acid with varying ratios of $\mathrm{N}_{2}: \mathrm{NH}_{4}{ }^{+}$was evaluated. Batch reactors $(500 \mathrm{ml})$ fitted with pressure gauges and a valve and septum arrangement were used for inoculation and incubation through the course of the experiment. Purging the reactors with nitrogen provided oxygen limited environment. At pre-determined incubation times, pressure change was determined and gas composition analyzed using TCD gas chromatography. The data was then used to calculate the methane production rate. Fluorescence microscopy was used to determine bacterial density in the reactor fluid. For the two feed (acetate) concentrations of $50 \mathrm{mM}$ (Ac1x) and $250 \mathrm{mM}$ (Ac5x), production profiles of methane (mol/L) vs. ammonium addition $\left(\mathrm{NH}_{4} \mathrm{Cl}\right)$ show reverse trends. Production profiles of carbon dioxide could be used to explain this opposing effect. Nitrogen fixation by the consortia was seen in cases of ammonium limitation. $\mathrm{NH}_{4} \mathrm{Cl}$ concentration appeared to benefit acetate-utilizing methanogens, but seemed to slightly inhibit $\mathrm{CO}_{2}$ utilizing methanogens. High carbon content reversed this inhibition. Also, both trends were reversed upon doubling the 
incubation time ( 27 vs. 56 days). Methane production was dependent on the $\mathrm{NH}_{4} \mathrm{Cl}$ concentration, carbon content, and residence time. Figures 2 and 3 show the effect of ammonium chloride concentration on the production of methane for both feed compositions of acetate (Ac1x and Ac5x). As can be seen for Ac1x, $150 \mathrm{mM}$ of $\mathrm{NH}_{4} \mathrm{Cl}$ gave the best yield. For the higher $\mathrm{NH}_{4} \mathrm{Cl}$ contents $(100$ and $150 \mathrm{mM})$, the methane production not only started earlier, but also retained the accelerated rate and gave very high yields. For secondary transfers, residence time was greatly reduced and the carbon dioxide trends obtained further verified the above observations.

For the case with Ac5x, high carbon content, $56 \mathrm{mM}$ gave the highest yield of methane, while the lowest yield was observed for $150 \mathrm{mM}$. The methanogens need a source of nitrogen. Comparing the 20 and $56 \mathrm{mM}$ samples with the 0 and $10 \mathrm{mM}$ samples, it was seen that if the nitrogen is provided through small quantities of ammonium chloride, the residence time could be considerably reduced. Nitrogen gas in the headspace can also be an effective source to fulfill the nitrogen requirement, but it appears that some ammonium chloride was responsible for quick initial methanogenic activity. Once the ammonium chloride is consumed, the reaction will progress if the acetate (carbon) content present is high enough to induce the methanogens to work. This is an ideal condition, since the energy required to maintain the biomass is supplemented by more electron transfer and thus more methane production. The rate remains accelerated, resulting in very high yields.

Very high amounts of ammonium chloride can also prove to be inhibitory, especially to the hydrogenotrophic methanogens. This could be the reason why for the 100 and the $150 \mathrm{mM}$ media samples, methane yield was not increased. It can also be seen that as the ammonium chloride concentration was raised beyond $20 \mathrm{mM}$, the residence time also increased, giving a further indication of inhibition.

For Ac1x (low carbon content), the highest yield was obtained at $150 \mathrm{mM}$, and lowest, at $56 \mathrm{mM}$. The trend of methane production versus ammonium chloride concentration was completely reversed at this feed concentration, compared to Ac5x. Reduced yield at $56 \mathrm{mM}$ could be due to the decreased carbon content. Even though, for 27 days, the case with $56 \mathrm{mM}$ gave a high yield, as seen before, after the ammonium chloride was consumed, the carbon content (acetate) was very low (50\% 
conversion already completed). Due to energy constraints, the methanogens were not forced to fix nitrogen from the headspace gas as seen for Ac1x.

Due to absence of ammonium chloride, samples run with 0 and $10 \mathrm{mM} \mathrm{NH}_{4} \mathrm{Cl}$ showed a lag before methane production started, but accelerated rapidly once the methanogens started fixing the nitrogen from the headspace. The final yield, though considerable, still remained lower than that for the 100 and $150 \mathrm{mM}$ cases. The tests with 100 and $150 \mathrm{mM} \mathrm{NH}_{4} \mathrm{Cl}$ also had a similar lag time, probably due to ammonium chloride inhibition. However, once the reaction started it accelerated very rapidly. The ammonium chloride content probably never became limiting. Thus the reaction did not really slow down until the carbon source was almost completely consumed.

The feed concentration also had an effect on the lag time of the reaction as well as the final percent conversion. The residence time was considerably increased, and the final percent conversion was lower for the higher carbon content. The carbon dioxide production shows clearly the inhibition of the methanogens due to high ammonium chloride concentration, and also suggest that the inhibition could be dependent on a $\mathrm{C}: \mathrm{N}$ ratio. Fluorescence microscopy experiments showed that the methanogenesis could be directly linked to the bacterial cell growth. The higher the cell growth, the higher will be the production of methane. 


\section{REFERENCES}

1. Ahring, B. K., Westermann, P., and Mah, R. A. 1991. Hydrogen inhibition of acetate metabolism and kinetics of hydrogen consumption and Methanosarcina thermophila TM-1. Arch. Microbiol. 157: p. 38-52.

2. Archer, D. B. 1984. Biochemistry of methanogenesis by mixed cultures. Biochemical Society transactions. 12: p. 1144-1146

3. Archer, D. B., and Kirsop, B.H. 1990. The microbiology and control of anaerobic digestion. Anaerobic Digestion: A Waste Treatment Technology, eds. Elsevier Applied Science, London and New York, p. 43-93.

4. Arora, D. et al.1997. Production of ethanol from refinery waste gas phase. DOE final report (DOE-AL-98770-3).

5. Baresi, L., Mah, R. A., Ward, D. M., and Issac, R. K. 1978. Methanogenesis from acetate: enrichment studies. Applied and Environmental Microbiology. 36(1): p. 186-197.

6. Barker, H. A. 1943. Studies on the methane fermentation VI. The influence of carbon dioxide concentration on the rate of carbon dioxide reduction by molecular hydrogen. Proc. Natl. Acad. Sci. U.S.A. 29: p. 184-190.

7. Belay, N., Sparling, R., and Daniels, L. 1984. Dinitrogen fixation by thermophilic methanogenic bacterium. Nature. 312: p. 286-288.

8. Jarell, K. F., and Saulnier, M. 1987. Inhibition of methanogenesis in pure cultures by ammonia, fatty acids, and heavy metals, and protection against heavy metal toxicity by sewage sludge. Can. J. Microbiol. 33(6): p. 551-4.

9. Klasson, K., Ackerson, M., Clausen, E., and Gaddy, J. 1993. Biological conversion of coal and coal-derived synthesis gas. Fuel. 72(12): p. 1673.

10. Krzycki, J. A., Morgan, J. B., Conrad, R., and Zeikus, J. G. 1987. Hydrogen metabolism during methanogenesis from acetate by Methanosarcina barkeri. FEMS Microbiol. 40: p. 193-198.

11. Lovely, D.R., and Ferry, J.G. 1985. Production and consumption of $\mathrm{H}_{2}$ during growth of Methanosarcina spp. on acetate. Applied and Environmental Microbiology. 48, 81-87.

12. Murray, A. P., and Zinder, S. H. 1984. Nitrogen fixation by a methanogenic archaebacterium. Nature. 312: p. 284-286.

13. Patel, G. B., and Sprott, G. D. 1990. Mathanosaetta concilii gen. nov., sp. nov. ("Methanothrix concilii") and Mathanosaetta thermoacetophila nom. rev., comb. nov. Int. J. Syst. Bacteriol. 40: p. 79-82.

14. Patel, G. B., and Sprott, G. D. 1991. Cobalt and sodium requirements for methanogenesis in washed cells of Mathanosaetta concilii. Can. J. Microbiol. 37: p. 110-115. 
15. Pienemann, S., Muller, V., Blaut, M., and Gottshalk, G. 1988. Bioenergetics of methanogenesis from actate by Mathanosarcina barkeri. J. Bacteriol. 170: p. 13691372.

16. Soubes, M. et al. 1994. Inhibition of methanogenesis from acetate by $\mathrm{Cr}^{+3}$ and ammonia. Urug. Biotechnol. Lett. 16(2): p. 195-200.

17. Van Den berg, L., and Saulnier, M. 1987. Inhibition of methanogenesis in pure cultures by ammonia, fatty acids, and heavy metals, and protection against heavy metal toxicity by sewage sludge. Can. J. Microbiol. 33(6): p. 551-4.

18. Wang, M., Saricks, C, and Wu, M. 1999. Fuel ethanol produced from midwest U.S. corn: help or hindrance to the vision of Kyoto? Journal of the Air \& Waste Management Association. 49(7): p. 756-772.

19. Zehnder, A. J. B., Huser, B. A., Brock, T. D. and Wuhrmann, K. 1980. Characterization of an acetate-decarboxylating, non-hydrogen-oxidizing methane bacterium. Archives of Microbiology. 124: p. 1-11.

20. Zinder, S. H., and Anguish, T. 1992. Carbon, monoxide, hydrogen and formate metabolism during methanogenesis from acetate by thermophilic culture of Methanosarcina and Methanothrix strains. Applied and Environmental Microbiology. 58(10): p. 3323-3329. 


\section{FIGURES}

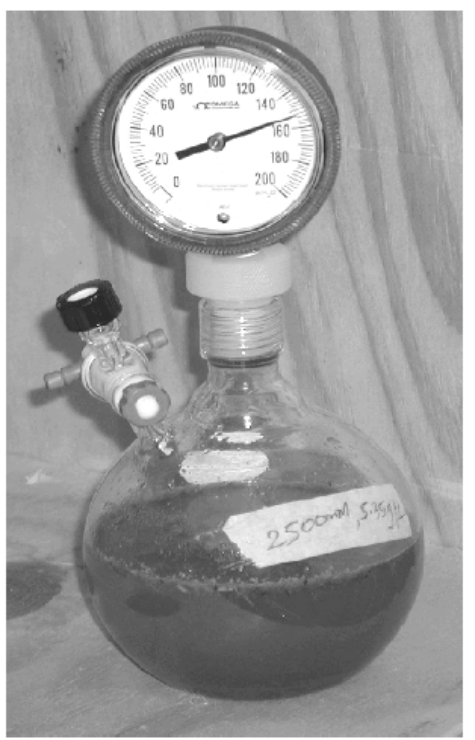

Figure $1.500 \mathrm{ml}$ custom built reactor.

\section{$\mathrm{CH}_{4}$ production for $\mathrm{Ac} 1 \mathrm{x}$}

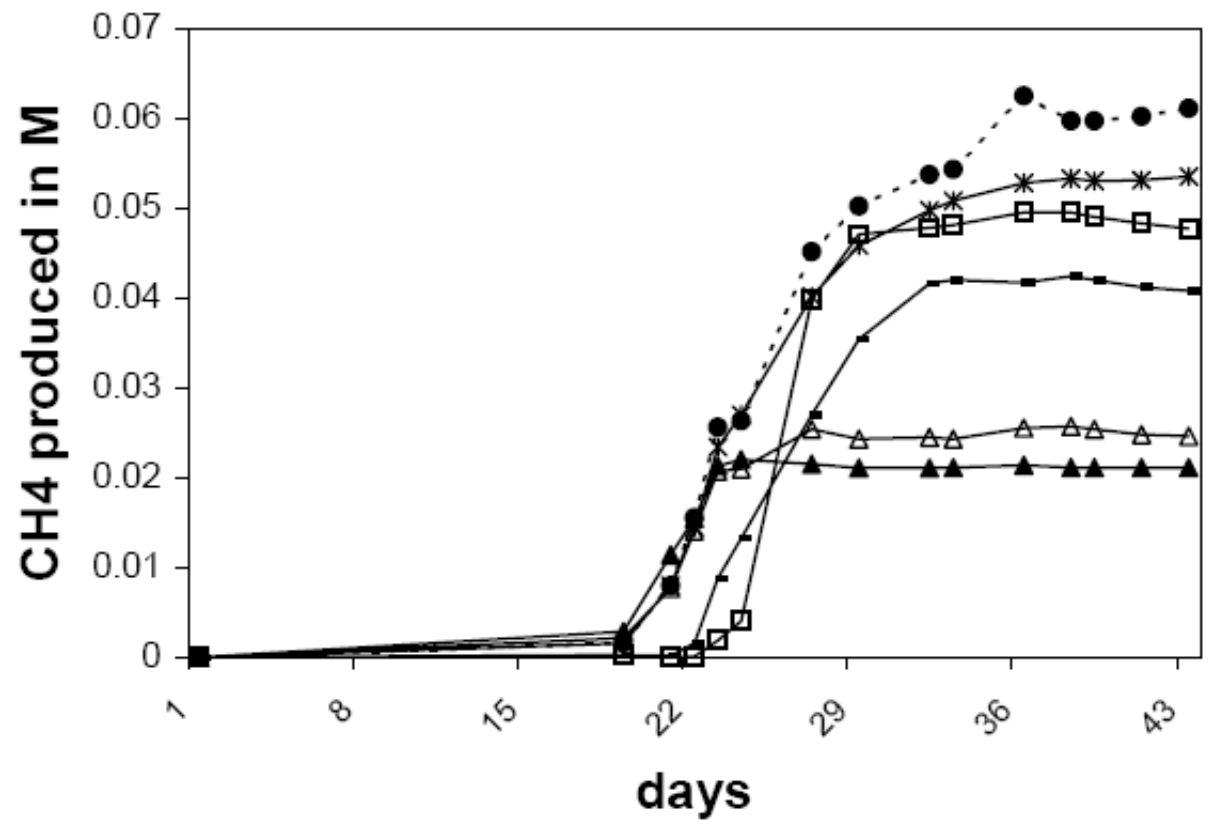

Figure 2. Effect of $\mathrm{NH}_{4} \mathrm{Cl}$ concentration on the production of methane for Ac1x.

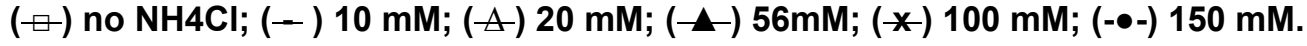




\section{$\mathrm{CH}_{4}$ production for $\mathrm{Ac5x}$}

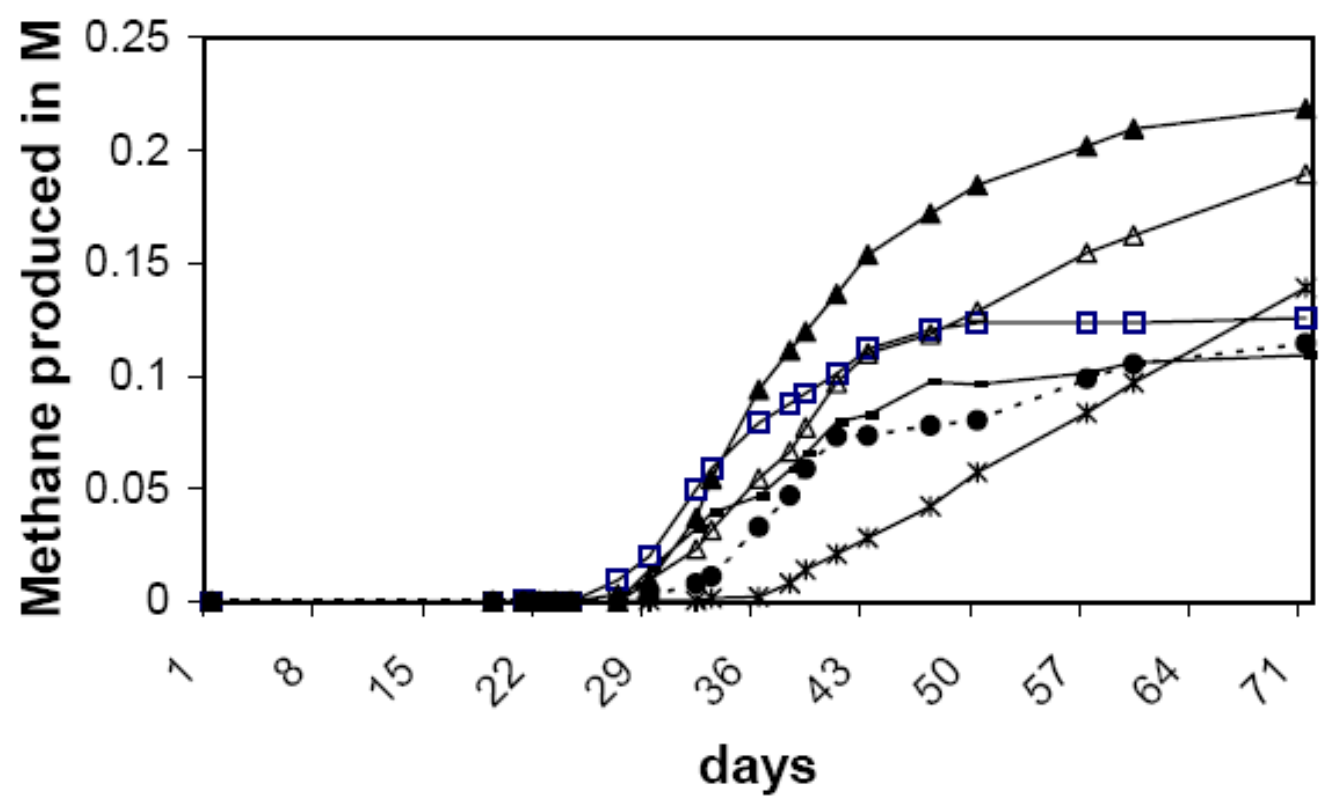

Figure 3. Effect of $\mathrm{NH}_{4} \mathrm{Cl}$ concentration on the production of methane for Ac5x.

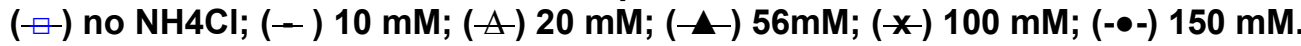




\section{TABLE}

Table 1. Screening of inoculates for methane production in serum bottles

\begin{tabular}{|c|c|c|c|c|c|}
\hline Inoculate source & $\begin{array}{c}\text { Residence time } \\
\text { average (day) }\end{array}$ & Trials & $\begin{array}{l}\text { Positive } \\
\text { results }\end{array}$ & Media/inoculate ${ }^{b}$ & $\%$ Inoculate \\
\hline Miscellaneous $^{a}$ & - & 8 & 1 & $38(115 / 3)$ & $2.6 \%$ \\
\hline $\begin{array}{l}\text { New York } \\
\text { sediment }\end{array}$ & 70 & 5 & 4 & $38(115 / 3)$ & $2.6 \%$ \\
\hline $\begin{array}{l}\text { New York } \\
\text { sediment }\end{array}$ & 48 & 5 & 4 & $15(15 / 1)$ & $6.67 \%$ \\
\hline Sardis sediment & - & 15 & 4 & $15(15 / 1)$ & $6.67 \%$ \\
\hline Deer dung & - & 5 & - & $15(15 / 1)$ & $6.67 \%$ \\
\hline Horse dung & 48 & 5 & 3 & $15(15 / 1)$ & $6.67 \%$ \\
\hline Cow dung & 27 & 5 & 3 & $15(15 / 1)$ & $6.67 \%$ \\
\hline Cow dung & $<27$ & 6 & 3 & $5(15 / 3)$ & $20 \%$ \\
\hline Cow rumen fluid & $<20$ & 9 & 6 & $5(15 / 3)$ & $20 \%$ \\
\hline
\end{tabular}




\section{METHANOGENESIS AT LOW pH IN LABORATORY SCALE BATCH FERMENTORS Performer: Mississippi State University}

\section{INTRODUCTION}

Methanogenesis is the process by which organic and inorganic compounds are converted to methane and carbon dioxide by anaerobic organisms known as methanogens. Autotrophic methanogens utilize $\mathrm{CO}_{2}$ and $\mathrm{H}_{2}$, while heterotrophic methanogens convert acetate, formate, and other compounds to support metabolic functions. Acetate is the most important substrate resulting in methane generation, accounting for about two-thirds of the methane produced in nature. The other third is produced from the reduction of $\mathrm{CO}_{2}$ with electrons derived from the oxidation of $\mathrm{H}_{2}$ or formate. Methane is produced from acetate via a fermentation process in which the acetate molecule is cleaved and the methyl group is reduced to methane with electrons derived from oxidation of the carbonyl group to $\mathrm{CO}_{2}$. This process is illustrated in the following reaction.

$$
\mathrm{CH}_{3} \mathrm{COO}^{-}+\mathrm{H}^{+} \rightarrow \mathrm{CH}_{4}+\mathrm{CO}_{2}
$$

Reaction 2 shows the process by which methanogens convert hydrogen and carbon dioxide to methane.

$$
4 \mathrm{H}_{2}+\mathrm{CO}_{2} \rightarrow \mathrm{CH}_{4}+2 \mathrm{H}_{2} \mathrm{O}
$$

Since 1974, new isolates of methanogens continuously have been obtained and described. Currently, Methanosarcina sp. and Methanosaeta sp. (formerly known as Methanothrix sp.) are identified as the only two known types of methanogens capable of metabolizing acetate [1]. However, it has been very difficult to isolate pure cultures of aceticlastic methanogens, likely due to close symbiotic relationships with other bacteria [2]. Methanosarcina sp. is a much more versatile genus of methanogens that includes species capable of growing on several different substrates including $\mathrm{H}_{2} / \mathrm{CO}_{2}$, methanol, methylamines, and acetate, while Methanosaeta sp. can only use acetate as an energy source [3].

One of the most important environmental factors associated with optimizing the methanogenic production of biogas is the system $\mathrm{pH}$. There is significant variation in the values reported in literature for the optimum $\mathrm{pH}$ required for methanogenesis. 
Methanosaeta sp. has been shown to produce methane in the range of $\mathrm{pH}$ 6.8-8.2. One study suggested an optimum range of $\mathrm{pH}$ 7.4-7.8 and reported no activity below $\mathrm{pH}$ 6.8 or above $\mathrm{pH} 8.2$ [3], while another reported the optimum pH for Methanosaeta sp. to be 7.8 with no activity below 6.8 [4]. Methanosarcina sp. has been shown to grow in a much wider range of $\mathrm{pH}$ 6.0-8.0 with the optimum $\mathrm{pH}$ around 7.0 [5]. Brummeler et al. [4] suggested that it can grow at a pH as low as 5.0. An enriched culture of methanogens was shown to produce methane in the range of $\mathrm{pH}$ 5.8-7.1; however, at $\mathrm{pH} 7.6$ there was no methane production [2]. Industrial and municipal anaerobic digesters have typically operated in the $6.0-7.0 \mathrm{pH}$ range and have noted significant problems with biomass retention and decreases in methane production at $\mathrm{pH}$ values less than about 6 [4] [6]. Speece recommends that the $\mathrm{pH}$ of the wastewater be maintained in the range of 6.8-7.0 by addition of alkalinity in the form of sodium carbonate, soda ash, caustic soda, and sodium bicarbonate [6]. As a result of these operational constraints, application of methanogenic digestion to low $\mathrm{pH}$ wastestreams has often been problematic and costly due to higher alkalinity supplementation requirements [16].

More recent research has begun to investigate and exploit the ability of some methanogens to tolerate and even thrive in acidic environments [7-10] [14-15]. The most notable example of acidophilic methanogenesis occurs in bogs and peatlands, where the bulk $\mathrm{pH}$ is often less than 5.0. It is estimated that about one-third of all methane produced in nature is derived from $\mathrm{H}_{2} / \mathrm{CO}_{2}$, with peatbogs and wetlands being the primary source of methane generated from these substrates [8]. While these environments are known to be a major source of atmospheric methane, contributing approximately $3-7 \%$ of the annual emissions of greenhouse methane, it has proven difficult to isolate methanogenic bacteria capable of growth at low $\mathrm{pH}[9]$. One early attempt to isolate an acidophilic methanogen failed; however, the research did show that the organism was capable of producing methane at pH 3.0-4.0 [14]. Further efforts to isolate acidophilic methanogens reported that two strains of Methanosarcina barkeri grew most rapidly at a $\mathrm{pH}$ of 5.0 when hydrogen and methanol were used as substrates [10]. Patel et al. successfully isolated a species of Methanobacterium able to grow and 
produce methane at a $\mathrm{pH}$ value as low 4.68 , with optimum growth occurring in the moderately acidophilic $\mathrm{pH}$ range of 5.6-6.2 [15].

One published hypothesis explaining the ability of methanogens to survive in acidic environments involves the ability of the organisms to control the $\mathrm{pH}$ of the surrounding microenvironment. It is possible that the methane produced in bogs and peatlands occurs in localized, higher $\mathrm{pH}$ microniches and within the neutral $\mathrm{pH}$ niche inside the microorganism itself [7]. Other research suggests that the ability of methanogens to function at low $\mathrm{pH}$ is dependent upon what substrate is being catabolized. Some strains of Methanosarcina have been shown to prefer acidic $\mathrm{pH}$ conditions when grown using $\mathrm{H}_{2}$ /methanol and $\mathrm{H}_{2} / \mathrm{CO}_{2}$ as substrates rather than acetate. However, when the catabolic substrate was changed to acetate, the growth was significantly slower at pH values below 7 [10]. Kim et al. also reported observing activity by hydrogen utilizing methanogens at $\mathrm{pH}$ values below 5.0 in batch systems designed for hydrogen production. However, in these same systems, aceticlastic methanogenic activity was inhibited [11].

While comparatively little is know about methanogenesis at low $\mathrm{pH}$, the published research suggests that there could be advantages to operating below the widely accepted optimal range of $\mathrm{pH} 6.0$ to 7.0 , including reducing or eliminating the need for alkalinity supplementation to buffer the digester $\mathrm{pH}$ and the potential increased production of methane over carbon dioxide in the resulting gas (aka biogas), which will increase the energetic value of the biogas. The goal of this research was to compare the methane production from a synthetic acetic acid wastewater stream in a batch reactor system with an initially low pH (4.5) to the results of methanogenesis using a neutral $\mathrm{pH}$ (7.0) solution without the addition of any supplemental alkalinity.

\section{METHODS AND MATERIALS}

\section{Reactor Design}

All experiments were conducted in $500 \mathrm{~mL}$ round, flat bottom flasks custom made by Ace Glass (Vineland, NJ, USA). Each flask had three threaded necks, and the total volume of the flasks ranged from 505 to $560 \mathrm{~mL}$. The center neck of each flask was fitted with an adapter consisting of a 1.5-2.0 mm glass capillary tube and a threaded bushing with a $1.5 \mathrm{~mm}$ bored hole. The capillary tube and bushing adapter 
was attached to the reactor through threaded nylon bushing with a $7.5 \mathrm{~mm}$ center hole. The capillary tube portion of the adapter fit inside the hole of the bushing and was sealed with an o-ring. The bushing was also sealed to the reactor with an o-ring. An Agilent long-life septa was placed between the bushing and capillary tube to facilitate gas sampling. A complete diagram of the reactors is shown in Figure 1.

One of the two larger side necks on the reactors was fitted with a solid threaded PTFE plug sealed with an o-ring. The second side neck was fitted with an Ashcroft test pressure gauge, used to monitor gas production and determine when gas and liquid sampling was necessary. Each Ashcroft pressure gauge (case type 1084) was 316 Stainless Steel and measured from 0-15 psig. Prior to conducting experiments, each of the reactors was pressure tested by injecting nitrogen into the reactor until the pressure gauge read approximately 10 psig. All of the fittings were checked for leaks, and pressurized reactors were incubated overnight to verify that the pressure could be maintained.

\section{Solution Preparation}

All of the acetic acid solutions were prepared using $97 \%$ (by weight) glacial acetic acid and distilled water. The initial acetic acid concentration in all experiments was 2 g/L. Nutrients were added to the acetic acid solution as recommended by Speece [6]. The nutrient solution contained $\mathrm{NH}_{4} \mathrm{Cl}(12.608 \mathrm{~g} / \mathrm{L}), \mathrm{KH}_{2} \mathrm{PO}_{4}(0.429 \mathrm{~g} / \mathrm{L}), \mathrm{Na}_{2} \mathrm{~S}_{2} \mathrm{O}_{3} \cdot 5 \mathrm{H}_{2} \mathrm{O}$ (2.554 g/L), $\mathrm{CaCl}_{2} \cdot 2 \mathrm{H}_{2} \mathrm{O}(0.477 \mathrm{~g} / \mathrm{L}), \mathrm{MgCl}_{2} \cdot 6 \mathrm{H}_{2} \mathrm{O}(0.151 \mathrm{~g} / \mathrm{L}), \mathrm{FeCl}_{2} \cdot 6 \mathrm{H}_{2} \mathrm{O}(0.0819 \mathrm{~g} / \mathrm{L})$, $\mathrm{NiCl}_{2} \cdot 6 \mathrm{H}_{2} \mathrm{O}(0.0162 \mathrm{~g} / \mathrm{L}), \mathrm{CoCl}_{2} \cdot 6 \mathrm{H}_{2} \mathrm{O}(0.0121 \mathrm{~g} / \mathrm{L})$, and $\mathrm{ZnCl}_{2}(0.0417 \mathrm{~g} / \mathrm{L})$, and one $\mathrm{mL}$ of nutrient solution was added for every gram of acetic acid per liter of solution. The $\mathrm{pH}$ of the combined acetic acid and nutrient solution was controlled using a solution of approximately $20 \%$ sodium hydroxide. Once the acetic acid and nutrient solution was prepared, the initial $\mathrm{pH}$ of the solution was adjusted to 4.5, 7.0, or 9.0 using the sodium hydroxide solution, a method similar to that used by Visser et al.[12], and Williams and Crawford $[13,14]$. All of the chemicals used to prepare the solutions were purchased from Fisher Scientific. 


\section{Experiment Preparation}

To maintain anaerobic conditions in the reactor, all experiments were prepared in an anaerobic flexible vinyl chamber (Coy Products, Grass Lake, MI, USA). The vinyl chamber atmosphere contained a 95\% nitrogen-5\% hydrogen gas mixture. To ensure that the solutions were anaerobic, the acetic acid nutrient solution was placed in the reactor in an open container and allowed to sit in the chamber for several hours. The open reactors were also placed in the chamber along with the necessary amount of digested sewage sludge required to inoculate the reactors. The digested sewage sludge was obtained from the Tuscaloosa, AL and the Vicksburg, MS sewage treatment plants. After equilibrating in the chamber, $20 \mathrm{~mL}$ of digester sludge and $250 \mathrm{~mL}$ of acetic acid nutrient solution were added to the reactors. The reactors were then sealed, removed from the anaerobic chamber, the pressure gauges were aligned to zero, and the reactors were incubated at $35^{\circ} \mathrm{C}$.

\section{Reactor Operation}

The reactors were incubated at $35^{\circ} \mathrm{C}$ for the entire length of each experiment. The pressure in the reactors was monitored, and experiments were deemed complete when the reactor pressure no longer increased, indicating cessation of gas production. When the pressure reached 10-15 psig, gas samples were collected using a $250 \mu \mathrm{L}$ gas-tight Luer Lok® syringe fitted with a 23 gauge needle. A $115 \mathrm{~mm}$ long needle was inserted through the bored hole in the bushing in the center sample port of the reactors. The needle length ensured that samples were collected from the headspace of the reactor and not from the capillary tube. After analyzing the gas, reactors were opened in the anaerobic chamber to release the pressure and collect liquid samples for $\mathrm{pH}$, redox potential, chemical oxygen demand (COD), and high pressure liquid chromatography (HPLC) analysis. Regarding the experiments with an initial $\mathrm{pH}$ of 4.5 , all of the experiments continued producing gas after the initial set of gas and liquid samples were collected. As a result, a second set of gas and liquid samples were collected for these experiments upon cessation of gas production. 


\section{Sample Analysis}

Gas and liquid samples from the reactors were analyzed for several different parameters. Gas samples were analyzed for methane and carbon dioxide using an Agilent $6890 \mathrm{~N}$ gas chromatograph (GC) with a thermal conductivity detector (TCD). A volume of $100 \mu \mathrm{L}$ of gas was injected into the GC. The column used to separate the methane and carbon dioxide was a Supelco 80/100 Porapak column. The column was 6 ' long by 1/8" ID and made of Stainless Steel. The standard curves for methane and carbon dioxide were prepared by injecting fixed amounts $(10,50$, and $100 \mu \mathrm{L})$ of pure gas and plotting the resulting response. The operating parameters of the GC were as follows: initial oven temperature of $100^{\circ} \mathrm{C}$ for 3 minutes, ramped to $150^{\circ} \mathrm{C}$ at $25^{\circ} \mathrm{C}$ per minute; front inlet temperature of $200^{\circ} \mathrm{C}$; front detector temperature of $250^{\circ} \mathrm{C}$; heater temperature of $250^{\circ} \mathrm{C}$; reference flow rate of $20 \mathrm{~mL}$ per minute; helium make-up flow rate of $5 \mathrm{~mL}$ per minute; column flow rate of $20 \mathrm{~mL}$ per minute; and total flow rate of $20.8 \mathrm{~mL}$ per minute.

Liquid samples were analyzed for Chemical Oxygen Demand (COD), acetic acid concentration, $\mathrm{pH}$ and redox potential. COD analysis was completed according to Hach Method 8000 for the $0-15,000 \mathrm{mg} / \mathrm{L}$ range (accuracy $\pm 100 \mathrm{mg} / \mathrm{L}$ ). A volume of $200 \mu \mathrm{L}$ of liquid sample was added to COD High Range Plus Digestion Reagent Vials, and the vials were incubated in a Hach COD Reactor at $150^{\circ} \mathrm{C}$ for 2 hours. Once the samples were completely digested and cooled, the COD concentration in $\mathrm{mg} / \mathrm{L}$ was measured using a Hach DR/2010 Spectrophotometer.

The acetic acid concentration in the liquid samples was measured using a Waters HPLC. Before sample analysis, each $1 \mathrm{~mL}$ volume of liquid sample was acidified with 4-5 drops of HPLC grade $85 \%$ o-phosphoric acid. Liquid samples that were not immediately analyzed were stored in the freezer at $0^{\circ} \mathrm{C}$. Just prior to analysis, liquid samples were filtered using a $1.0 \mathrm{~mL}$ Luer Lok® syringe and a $25 \mathrm{~mm}$ syringe filter containing 0.2-0.6 $\mu \mathrm{m}$ pore size filter paper. The HPLC was fitted with a $4 \times 23 \mathrm{~mm}$ guard column followed by a Waters YMC ODS-AQ column specific for carboxylic acids. The operating parameters of the HPLC were as follows: Mobile phase of $20 \mathrm{mM} \mathrm{PO}_{4}$ at 
$\mathrm{pH} 3.5$; temperature of $30^{\circ} \mathrm{C}$; flow rate of $0.7 \mathrm{~mL} / \mathrm{min}$; injection volume of $10 \mu \mathrm{L}$; and a $220 \mathrm{~nm}$ UV and 0.08 AUFS detector/sensor,

An Accumet portable $\mathrm{pH} / \mathrm{mV}$ meter and $\mathrm{pH}$ probe were used to measure the $\mathrm{pH}$ of the liquid. The unit was calibrated using a two-point calibration procedure (Fisher Scientific brand $\mathrm{pH}$ buffers).

\section{RESULTS}

The goal of these experiments was to evaluate how the initial $\mathrm{pH}$ of the reactor system affects biogas production; therefore, this data reflects the results of a series of experiments containing a $2 \mathrm{~g} / \mathrm{L}$ acetic acid and nutrient solution with initial $\mathrm{pH}$ values of 4.5 and 7.0. The acid solution was not buffered; therefore, the degradation of acetic acid was accompanied by a rise in $\mathrm{pH}$. Not buffering the test solution allowed this research to study the chemodynamics associated with biogas production versus cell adaptation to low $\mathrm{pH}$ environments and resulting $\mathrm{pH}$ shift as acetic acid is degraded.

\section{Gas Production}

Reactor pressure was monitored continuously during the length of the experiment, and the experiment was deemed complete when the pressure in the reactor no longer increased over a period of at least one day. Once gas production commenced, the experiments with an initial $\mathrm{pH}$ of 4.5 and 7.0 performed comparably, gaining an average of 0.966 and 1.025 psig per day, respectively. On average, the $\mathrm{pH}$ 7.0 experiments required 15 days to complete, ceasing gas production at about 10 psig. The $\mathrm{pH} 4.5$ experiments required 33 days to complete. The additional time required to complete the $\mathrm{pH} 4.5$ experiments is partly due to a longer lag period observed prior to the onset of gas production, which averaged 13 days but ranged from about 3 to 29 days. In comparison, the $\mathrm{pH} 7.0$ experiments had an average lag period of less than 2 days. The extended lag period was likely the result of the cells requiring an acclimation period to adjust to the low $\mathrm{pH}$ environment. Additionally, experiments with an initial $\mathrm{pH}$ of 4.5 continued to produce varying amounts of gas for an additional 2-10 days after the initial set of gas and liquid samples were collected. The additional pressure increase ranged from 1.3-10.9 psig, and second set of gas and liquid samples were collected when reactor pressure remained constant. The pH 4.5 experiments produced a total 
amount of gas equivalent to an average of 16.1 psig. Even though gas production in the $\mathrm{pH} 4.5$ experiments continued, analysis of the GC data revealed that an average of $70 \%$ of the total methane production occurred before the first set of samples was collected, corresponding to an average time span of 23 days.

\section{System pH}

The initial $\mathrm{pH}$ of the acid solution had a noticeable effect on the final $\mathrm{pH}$ of the system, as shown in Figure 2. Methanogenesis in experiments with an initial pH of 7.0 resulted in an average final $\mathrm{pH}$ of 8.14. The experiments with an initial $\mathrm{pH}$ of 4.5 exhibited an average $\mathrm{pH}$ of 6.79 following production of approximately $70 \%$ of the total methane yield. The remaining $30 \%$ of methane production resulted in a final $\mathrm{pH}$ of 7.24. Since these experiments were not buffered, the increase in $\mathrm{pH}$ was expected as conversion of acetic acid to methane proceeded. The system $\mathrm{pH}$ has the most impact on the composition of the biogas. Figure 3 shows the distribution of carbonic acid, carbonate, and bicarbonate as a function of the system $\mathrm{pH}$. At $\mathrm{pH}$ values less than about 5 , carbon dioxide exists in solution as a mixture of primarily gas with some carbonic acid. As the $\mathrm{pH}$ increases to neutral and above, nearly all of the carbon dioxide/carbonic acid is converted to carbonate and/or bicarbonate. Since autotrophic methanogensis uses hydrogen and carbon dioxide and not carbonates, any increase in the amount of soluble carbon dioxide as carbonic acid results in more methane produced. In these experiments, a lower system $\mathrm{pH}$ inhibited the production of carbonates and allowed for more carbon dioxide to remain detectable via GC in the gas headspace.

\section{Gas Yield}

The most noticeable impact of lowering the initial $\mathrm{pH}$ of the system is evident in the gas yield. Figure 4 shows the methane yield and total gas yield for each of the three initial $\mathrm{pH}$ conditions. When the initial $\mathrm{pH}$ was 4.5 , the average total gas yield increased by about $80 \%$. As mentioned above, this increase is due to the fact that more $\mathrm{CO}_{2}$ remained in the headspace at lower system $\mathrm{pH}$ values, whereas the $\mathrm{CO}_{2}$ was converted to bicarbonates in the higher $\mathrm{pH}$ experiments. Even more important, however, is the increase in the methane yield during the initial period when the $\mathrm{pH}$ was 4.5. The lower initial $\mathrm{pH}$ increased the methane yield by an average of $30 \%$. The stoichiometric yield 
for methanogenesis from acetate is 1 mole each of methane and carbon dioxide per mole of acetic acid. On average, the experiments with an initial pH of 4.5 produced 1.3 moles of methane per mole of acetic acid consumed. The increase in methane yield is probably due to the increase in aqueous carbon dioxide at lower $\mathrm{pH}$ values. This carbon dioxide was then converted to methane by unicarbonic $\mathrm{CO}_{2}$ and $\mathrm{H}_{2}$ utilizing methanogens present in the system.

\section{Acetic Acid Degradation}

The acid concentration in the liquid phase was monitored using both COD and HPLC analysis. The experiments with initial $\mathrm{pH}$ values of 4.5 and 7.0 performed comparably, reducing the overall COD to about $500 \mathrm{mg} / \mathrm{L}$. The final COD concentrations were comparable to concentrations measured in control experiments that contained inoculum but no acetic acid. The COD results suggest that in these experiments, nearly all of the acetic acid was converted to methane, and the remaining COD can be attributed to cell material in the samples. The COD results were confirmed using HPLC analysis, which reported an acetic acid concentration of less than 200 ppm for both initial $\mathrm{pH}$ values. Table 1 summarizes the complete results of these experiments as discussed above.

\section{DISCUSSION AND CONCLUSIONS}

The data collected from these batch system experiments show that reducing the initial $\mathrm{pH}$ of the acetic acid substrate from 7.0 to 4.5 increases the methane yield and total gas yield by $30 \%$ and $80 \%$, respectively. The difference in methane yield between the experiments at an initial $\mathrm{pH}$ of 4.5 and 7.0 is statistically significant at the $99 \%$ confidence interval. Additionally, the methane yield at the low initial $\mathrm{pH}$ is $30 \%$ above the stoichiometric yield for this process. These findings seem to contradict the widely accepted condition that methanogenesis requires a $\mathrm{pH}$ range of 6.0-7.0 for methane production to occur [3-4] [6] [2]. However, they do coincide with limited research findings suggesting that some methanogens may acclimate to or even prefer a more acidic $\mathrm{pH}$, including work published by Jain and Mattiasson [16] and Kim et al. [11].

The lag period observed in these low pH experiments is likely attributable to the fact that the digester from which the source organisms were obtained operated at a 
neutral $\mathrm{pH}$. As a result, the organisms required a longer acclimation period before beginning gas production. These observations are also comparable to results published by Jain and Mattiasson [16]. Using activated sludge as seed, they reported methane production at $\mathrm{pH}$ values of $6.0,5.5,5.0$, and 4.5 , and 4.0. To achieve acidic conditions, the $\mathrm{pH}$ of their system was lowered step-wise from $\mathrm{pH} 7.0$ to $\mathrm{pH} 4.0$ in 0.5 increments. Initially, decreases in methane production were observed, but after some acclimation, methane production resumed to levels observed at higher $\mathrm{pH}$ values. However, they also reported much longer periods of methane production inhibition when the system $\mathrm{pH}$ was directly changed from 7.0 to 4.5 or 4.0 rather than decreased incrementally [16].

The $30 \%$ increase in methane yield above what is stoichiometrically expected from the methanogenic degradation of acetic acid is likely due both to an increase in the aqueous carbon dioxide present in the system and to the combined effects of the autotrophic and the heterotrophic methanogens present in the consortium. As reported in Table 1, when the initial $\mathrm{pH}$ was 4.5 , methanogenic conversion of the acetic acid substrate to produce $70 \%$ of the total methane yield increased the $\mathrm{pH}$ to an average of 6.79. In comparison, the system with an initial $\mathrm{pH}$ of 7.0 had a final $\mathrm{pH}$ of 8.14. The difference in the system $\mathrm{pH}$ range has a significant effect on the distribution of the carbonate system. This is especially true in the $\mathrm{pH}$ range of about 5.0-7.0, where just a 0.5 unit increase in $\mathrm{pH}$ can decrease the amount of aqueous carbon dioxide by over $40 \%$. At $\mathrm{pH}$ values of 7.0 and above, less than $10 \%$ aqueous carbon is present, whereas at a pH of 6.5 the fraction is about $40 \%$, The data obtained from experiments conducted at an initial $\mathrm{pH}$ of 4.5 suggest that the lower initial $\mathrm{pH}$ reduced the amount of carbon dioxide dissolved as bicarbonates in the liquid phase. As a result, the carbon dioxide gas distributed between the aqueous and gas phases, producing a two-fold effect on biogas production: 1) more carbon dioxide remained in the gas phase, increasing the total gas yield; and 2) the aqueous carbon dioxide in the liquid phase was converted to methane by the autotrophic methanogens. Bhadra, et al., noted the importance of dissolved $\mathrm{CO}_{2}$ to methane production and postulated a new mechanism predicting that the reduction of $\mathrm{CO}_{2}$ to form methane is an intracellular occurrence that contributes up to $28 \%$ of the total methane produced [17]. 
When considering the theoretical yield of this process, each mole of acetic acid added to the system should produce one mole of methane. The increase to about 1.3 moles of methane produced per mole of acetic acid in these experiments can likely be attributed to the autotrophic conversion of carbon dioxide (in the presence of $5 \%$ hydrogen from the anaerobic chamber) to methane. Evaluating these results and considering previous work with pure and mixed methanogenic cultures at low $\mathrm{pH}$, it can also be concluded that in this system, the autotrophic methanogens prefer a more acidic $\mathrm{pH}$ range, while the heterotrophic methanogens dominate gas production at neutral $\mathrm{pH}$ [16] [11] [10]. This conclusion is also supported by research indicating that the conversion of $\mathrm{H}_{2} / \mathrm{CO}_{2}$ is the predominate mechanism for the production of methane in naturally occurring acidic environments such as peat bogs [8]. It is important to note that these conclusions are with respect to measurements of the bulk $\mathrm{pH}$. The extent to which methanogens adjust the $\mathrm{pH}$ of their localized microenvironments to offset the effect of an acidic bulk pH is unknown; however, maintaining even a moderately acidic bulk $\mathrm{pH}$ is advantageous to maximizing the amount of carbon dioxide available for the autotrophic production of methane.

The results of these experiments show that a mixed culture of methanogens has the ability to acclimate to a lower $\mathrm{pH}$ environment, as the experiments with an initial $\mathrm{pH}$ of 4.5 produced $70 \%$ of the total methane yield in an environment ranging from acidic to moderately acidic. Most notably, however, a reduction in the initial pH (from 7.0 to 4.5) of a batch system supporting methanogenesis increased the methane yield by $30 \%$. These findings show that in addition to being an effective method for wastewater treatment, methanogenic digestion has the potential to be an even more significant source of methane, particularly for streams with a low influent $\mathrm{pH}$. Implementation of these results could improve the economic sustainability and broaden the use of methanogenic digesters for the conversion of municipal, industrial, and agricultural wastes to fuel. Along with increasing overall methane production, operating at a lower $\mathrm{pH}$ could lower or possibly eliminate the need for alkalinity supplementation, which can be a large expense, especially when treating dilute or acidic wastewaters [6]. Future work, critical to further understanding and optimizing this process, will investigate the feasibility of maintaining a semi-continuous laboratory scale methanogenic digester at 
low $\mathrm{pH}$. In particular, future experiments will include continuous monitoring of system $\mathrm{pH}$ in order to more precisely relate methane production to system $\mathrm{pH}$. 


\section{REFERENCES}

1. Ferry, J. (1992). Methane from acetate. J. Bacteriol., 174, 17, 5489-5494.

2. Van den Berg, L., Patel, G., Clark, D., Lentz, C. (1976). Factors affecting rate of methane formation from acetic acid by enriched methanogenic cultures. Can. J. Microbiol., 22, 1312-1319.

3. Huser, B., Wuhrmann, K., Zehnder, A. (1982). Methanothrix soehngenii gen. nov. sp. nov., a new acetotrophic non-hydrogen-oxidizing methane bacterium. Arch. Microbiol., 132, 1-9.

4. Brummeler, E., Hulshoff Pol, L., Dolfing, J., Lettinga, G., Zehnder, A. (1985). Methanogenesis in an upflow anaerobic sludge blanket reactor_at ph 6 on an acetate-propionate mixture. Appl. Environ. Microb., 49, 6, 1472-147̄7.

5. Yang, S., Okos, M. (1987). Kinetic study and mathematical modeling of methanogenesis of acetate using pure cultures of methanogens. Biotechnol. Bioeng., 30, 661-667.

6. Speece, R. (1996). Anaerobic biotechnology for industrial wastewaters. Nashville, TN: Archae Press.

7. Duval, B., Goodwin, S. (2000). Methane production and release from two New England peatlands. Internatl. Microbiol., 3, 89-95.

8. Goodwin, S., Zeikus, G. (1987). Ecophysiological adaptations of anaerobic bacteria to low ph: analysis of anaerobic digestion in acidic bog sediments. Appl. Environ. Microb., 53, 1, 57-64.

9. Horn, M., Matthies, C., Kusel, K., Schramm, A., Drake, H. (2003). Hydrogenotrophic methanogenesis by moderatly acid-tolerant methanogens of a methane-emitting acidic peat. Appl. Environ. Microb., 69, 1, 74-83.

10. Maestrojuane, G., Boone, D. (1991). Characterization of methanosarcina barkeri $\mathrm{ms}^{\mathrm{t}}$ and 227 , methanosarcina mazei $\mathrm{s}-6^{\mathrm{t}}$, and methanosarcina vacuolata $\mathrm{Z}-761^{\top}$. Int. J. Syst. Bacteriol., 41, 2, 267-274.

11. Kim, I., Hwang, M., Jang, N., Hyun, S., Lee, S. (2004). Effect of low ph on the activity of hydrogen utilizing methanogen in bio-hydrogen process. Int. J. Hydrogen Energ., 29, 1133-1140.

12. Visser, A., Gao, Y., Lettinga, G. (1993). Effects of ph on methanogenesis and sulphate reduction in thermophilic $\left(55^{\circ} \mathrm{C}\right)$ UASB reactors. Bioresource Technol., 44, 113-121.

13. Williams, R., Crawford, R. (1984). Methane production in minnesota peatlands. Appl. Environ. Microb., 47, 6, 1266-1271.

14. Williams, R., Crawford, R. (1985). Methanogenic bacteria, including an acidtolerant strain, from peatlands. Appl. Environ. Microb., 50, 6, 1542-1544. 
15. Patel, G., Sprott, G., Fein, J. (1990). Isolation and characterization of methanobacterium espanolae sp. nov., a mesophilic, moderately acidiphilic methanogen. Int. J. Syst. Bacteriol., 40, 1, 12-18.

16. Jain S., Mattiasson B. (1998). Acclimatization of methanogenic consortia for low ph biomethanation process. Biotechnol. Lett., 20, 8, 771-775.

17. Bhadra, A., Mukhopadhyay, S., Ghose. T. (1984). A kinetic model for methanogenesis of acetic acid in a multireactor system. Biotechnol. Bioeng., 26, 257-264. 


\section{FIGURES}

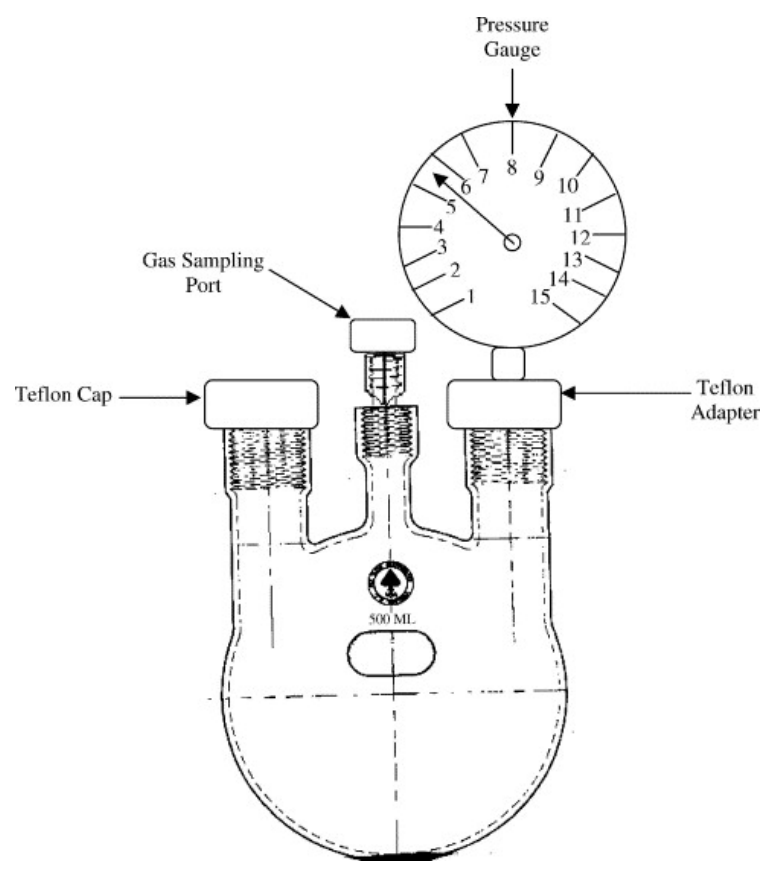

Figure 1. Diagram of Reactor.

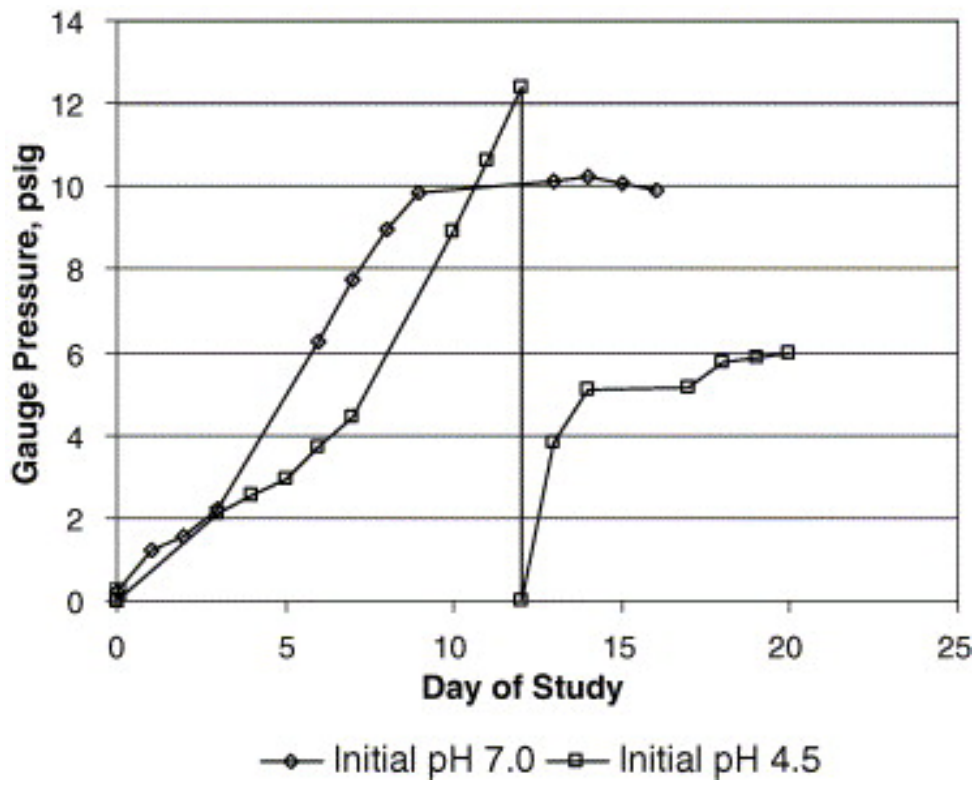

Figure 2. Effect of acid solution on the final pH of the system. 


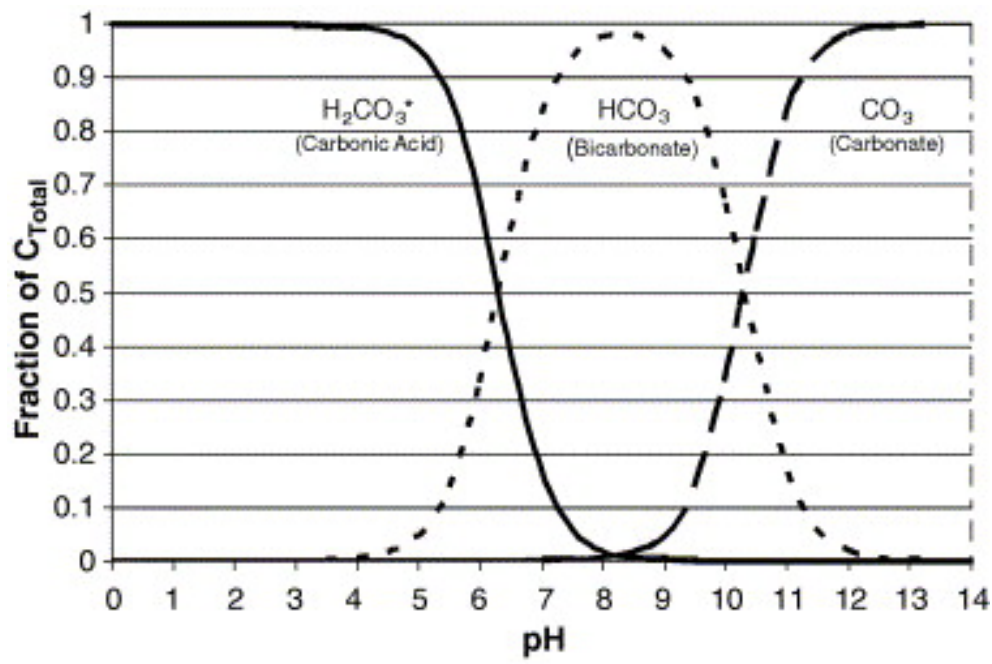

$\mathrm{C}_{\text {Toldal }}=\mathrm{H}_{2} \mathrm{CO}_{3}{ }^{*}+\mathrm{HCO}_{3}{ }^{-}+\mathrm{CO}_{3}^{2}$

At $\mathrm{pH}<5$, solution is approx. $0.16 \% \mathrm{H}_{2} \mathrm{CO}_{3}{ }^{*}$, the remainder is $\mathrm{CO}_{2}$

Figure 3. Distribution of carbonic acid, carbonate, and bicarbonate as a function of the system $\mathrm{pH}$

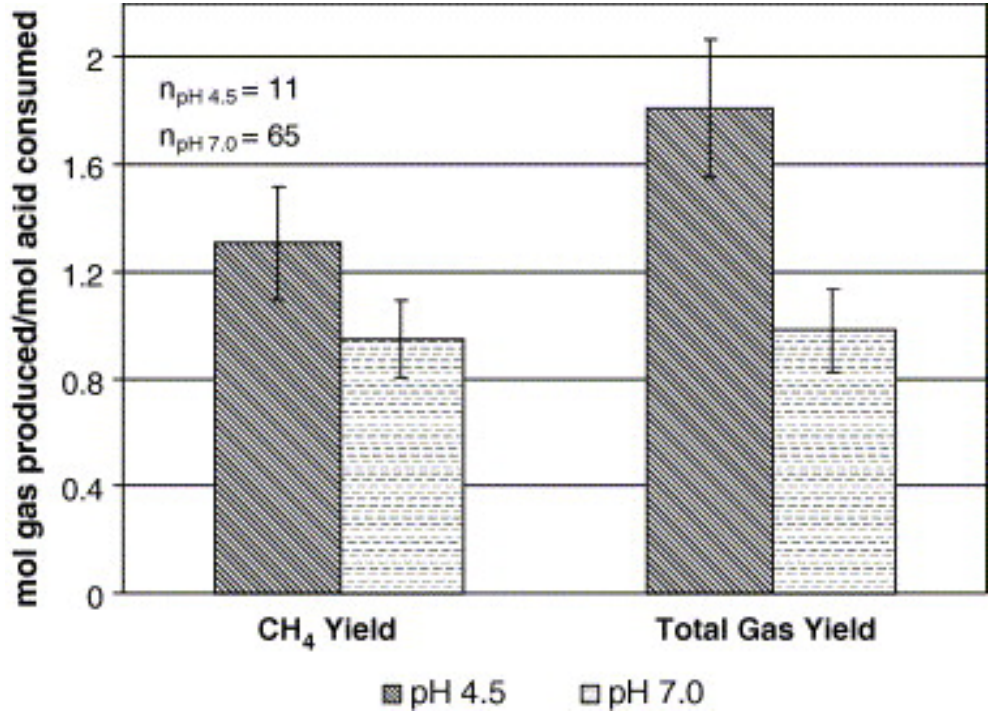

Figure 4. Methane yield and total gas yield for each of the three initial pH conditions 


\section{TABLE}

Table 1. Summary of results.

\begin{tabular}{|c|c|c|c|}
\hline & \multicolumn{2}{|c|}{ Initial pH $4.5( \pm 0.02)$} & Initial pH $7.0( \pm 0.11)$ \\
\hline \multirow[t]{2}{*}{ Lag period } & \multicolumn{2}{|l|}{ 3-29 days } & $<2$ days \\
\hline & \multicolumn{2}{|c|}{ Avg $=13$ days } & \\
\hline Average length of experiment & \multicolumn{2}{|l|}{33 days } & 15 days \\
\hline$\%$ Total $\mathrm{CH}_{4}$ production & $70 \%$ & $100 \%$ & $100 \%$ \\
\hline Final $\mathrm{pH}$ & $6.79 \pm 0.15$ & $7.24 \pm 0.39$ & $8.14 \pm 0.22$ \\
\hline Average initial COD (mg/L) & \multicolumn{2}{|l|}{3280} & 2990 \\
\hline Average final COD (mg/L) & \multicolumn{2}{|l|}{610} & 510 \\
\hline Average final (acid) & \multicolumn{2}{|l|}{$<200$ ppm } & $<200$ ppm \\
\hline Total $\mathrm{CH}_{4}$ yield (per mol acid) & \multicolumn{2}{|l|}{1.3} & 0.95 \\
\hline Total gas yield (per mol acid) & \multicolumn{2}{|l|}{1.8} & 0.98 \\
\hline
\end{tabular}




\section{EFFECTS OF SELECTED BY-PRODUCTS OF AN ACID HYDROLYZATE ON CELL GROWTH AND ETHANOL FERMENTATION BY SACCHAROMYCES CEREVISIAE Performer: Jackson State University}

\section{INTRODUCTION}

Acid Hydrolysis of lignocellulosic biomass results in the release of various sugars as well as inhibitory compounds such as furfural, 5-hydroxymethylfurfural (HMF), vanillin, and vanillic acid that could inhibit microbial growth and ethanol fermentation. During the process of acid hydrolysis of pinewood sawdust conducted in this study, heavy metals such as chromium $(\mathrm{Cr})$ were released from the extruder of the processing reactor. The purpose of this research was to study the effect of selected by-products in an acid hydrolyzate on cell growth and ethanol fermentation of Saccharomyces cerevisiae. Furfural, HMF, vanillin, vanillic acid, and $\mathrm{Cr}$ were added at different concentrations to fermentation media. S. cerevisiae growth and ethanol yield were measured after 18 hours of incubation. The results showed that individual presence of furfural, HMF, vanillin, and vanillic acid at concentrations as low as 1.0, 2.0, 2.0, and 0.5 $\mathrm{mg} / \mathrm{ml}$, respectively, significantly inhibited ethanol production, while $\mathrm{Cr}$ did not show any inhibitory effect at concentrations up to $20 \mathrm{ppm}$. Effects of the mixture of furfural, HMF, vanillin, vanillic acid, and $\mathrm{Cr}$ on ethanol yield and cell growth were also studied. Results showed that at low concentration such as $0.5 \mathrm{mg} / \mathrm{ml}$, the by-products stimulated not only the $S$. cerevisiae growth but also the ethanol production. At $1.0 \mathrm{mg} / \mathrm{ml}$, ethanol production and cell growth were significantly inhibited. Co-existence of $\mathrm{Cr}$ neither enhanced nor reduced the inhibitory/ stimulating effect.

There is a growing demand for the already depleting energy resources for fuel. One of the alternative solutions to the problem is ethanol production, which is a cost effective and environmentally friendly process of producing fuel (Luo et al., 2002). Processing method and feedstock are the determining factors in ethanol production costs. Currently in the U.S., corn is the primary feedstock for ethanol production and accounts for $60 \%$ of the total production costs [12]. The use of feedstock, such as lignocellulosic biomass, that has no or low commercial value could significantly reduce ethanol production costs. The processing of lignocellulosic biomass to obtain the monomeric sugars needed for fermentation is one of the major factors contributing to 
the non-competitive costs of lignocellulose derived ethanol. The conversion of the polysaccharides in lignocellulose to monosaccharides can be achieved by dilute or concentrated acid hydrolysis. The drawback of acid hydrolysis is that it produces a product (acid hydrolyzate) that contains not only the sugars needed for fermentation but also furan and phenolic compounds as well as weak acids that could inhibit fermentation and microbial growth.

The known by-product compounds found in acid hydrolyzate include furfural and 5-hydroxymethyl furfural (HMF) formed from high temperature degradation of pentose and hexose sugars respectively [13] [18] [21-22]. Others include phenolics formed from the partial breakdown of lignin [1] [11] [13] and weak organic acids such as acetic acid as well as formic and levulinic acids that are formed from the breakdown of HMF and furfural [13] [15] [16]. The presence of furfural at $1 \mathrm{mg} / \mathrm{ml}$ was reported to decrease the $\mathrm{CO}_{2}$ evolution [20], the cell multiplication [3], and the total viable cell [7] in the early phase of fermentation by yeast. HMF was an inhibitor to the growth of $S$. cerevisiae, but not as strong as furfural [20]. An early study showed that HMF at the concentration of $1 \mathrm{mg} / \mathrm{ml}$ had an inhibitory effect on baker's yeast [10]. A more recent study reported that only at higher concentration $(2 \mathrm{mg} / \mathrm{ml})$, HMF decreased bacterial biomass yield by $23 \%$ [6]. Vanillin was one of the strongest inhibitors among the hydrolysis by-products [9]. It significantly inhibited ethanol production by Saccharomyces carlsbergensis W34 at $5 \mathrm{mg} / \mathrm{ml}$ [19]. When the concentration was $<1 \mathrm{mg} / \mathrm{ml}$, vanillic acid had no effect on ethanol fermentation by S. cerevisiae [1].

To extract sugars from lignocellulose of pinewood sawdust in this study, a twinscrew extruder reactor was used for continuous impregnation with sulfuric acid. In our previous study [24], we reported that the growth and fermentation ability of $S$. cerevisiae were severely inhibited by undiluted and slightly diluted (eg., $100 \mathrm{X}$ ) acid hydrolyzates. Extreme acidity was speculated to be a major reason for the inhibition. Recently we found out that besides various organic by-products, certain heavy metals such as chromium ( $\mathrm{Cr}$ ) were also released into the hydrolyzate due to acid corrosion of stainless steel of the extruder. Indeed, we found that there was up to $16 \mathrm{ppm}$ of $\mathrm{Cr}$ present in the hydrolyzate. 
Although studying the inhibitory effect of individual by-products is important for formulating hydrolyzate production, synergism could occur due to the presence of multiple by-products in most hydrolyzates. Therefore, it is also important to study the effect of the presence of multiple by-products on cell growth and ethanol fermentation by S. cerevisiae. The objectives of this study were: (1) to determine the effect of individual organic by-products and chromium on cell growth and ethanol fermentation of S. cerevisiae; and (2) to determine the effect of the by-products mixture on cell growth and ethanol fermentation of $S$. cerevisiae.

\section{MATERIALS AND METHODS}

\section{Chemicals and Microorganisms}

2-Furaldehyde (furfural), 5-hydroxymethylfurfural (HMF), vanillin, vanillic acid, and water (HPLC grade) were purchased from Sigma Chemical Co. (St. Louis, MO). Chromium nitrate $\left[\mathrm{Cr}\left(\mathrm{NO}_{3}\right)_{3} \mathrm{9H}_{2} \mathrm{O}\right]$ was purchased from Fisher Scientific (Houston, $\mathrm{TX}$ ). The fermentation medium (Atlas, 1995) was prepared and consisted of $1 \%$ trypticase, $0.1 \%$ beef extract, $0.5 \%$ dextrose and $0.5 \% \mathrm{NaCl}(\mathrm{pH} \mathrm{6.4)}$. To prepare the inoculum, $S$. cerevisiae (ATCC \# 765) cells were incubated in potato dextrose broth for $18 \mathrm{~h}$ at $28^{\circ} \mathrm{C}$, on a rotary shaker at $200 \mathrm{rpm}$.

\section{Effects of the By-products on Growth and Ethanol Production}

Furfural, HMF, vanillin, vanillic acid, and chromium were detected in our hydrozylates (unpublished data). To study the effects of these individual organic byproducts on the growth and ethanol fermentation, $10 \mathrm{ml}$ of $\mathrm{S}$. cerevisiae cell suspension containing about $10^{7}$ cells were transferred to a 250-ml flask containing $100 \mathrm{ml}$ fermentation medium. Furfural, HMF, vanillin, and vanillic acid were added separately to achieve the final furfural concentrations of $0,1,2$, and $4 \mathrm{mg} / \mathrm{ml}$, plus final $\mathrm{HMF}$, vanillin, and vanillic acid concentrations of $0,0.5,1$, and $2 \mathrm{mg} / \mathrm{ml}$. Chromium nitrate was added to achieve the final chromium concentrations of $0,5,10$, and $20 \mathrm{ppm}$. The flasks were incubated at $30^{\circ} \mathrm{C}$ for 18 hours before samples were withdrawn for cell count and ethanol concentration analysis. To study the effects of the organic by-products mixture on the growth and ethanol fermentation, different amounts of furfural, HMF, vanillin, and vanillic acid were added together to achieve the final concentrations of $0,0.5$, or 1 
$\mathrm{mg} / \mathrm{ml}$ each of the organic by-products in the mixture solution. The chromium nitrate was also added to achieve the final concentrations of 0,8 , and $16 \mathrm{ppm}$. In this mixture, S. cerevisiae was inoculated and samples were incubated as described in the aforementioned procedure.

The colony count was carried out on potato dextrose agar plates (Benson, 1994). The ethanol concentration was determined by gas chromatography (Shimadzu GC-14A, Shimadzu Corp., Kyoto, Japan) equipped with a flame ionization detector. The oven temperature was $125^{\circ} \mathrm{C}$ and injector temperature was $230^{\circ} \mathrm{C}$.

\section{Chromium Analysis}

The paste from the extruder was treated with water in a 1:1 weight ratio and filtered using a glass Buchner funnel having 10-20 $\mu \mathrm{m}$ porocity. The chromium concentration in the filtrate was analyzed by atomic absorption spectroscopy (Shimadzu AA-6701F Atomic Absorption Flame Emission Spectrophotometer). Several standard solutions of various concentrations were prepared for quantitative measurement of chromium concentrations.

\section{Statistical Analysis}

Samples were run in triplicate. All results are expressed as mean \pm SD. Differences between treatment groups were tested by one-way analysis of variance (ANOVA) using the SAS System for Windows (V8, SAS Institute Inc., Gray, NC). Differences at $p<0.05$ are considered significant.

\section{RESULTS AND DISCUSSION}

To maximize ethanol production from fermenting sugars in the acid hydrolyzate by $S$. cerevisiae, the by-products from acid hydrolysis of lignocellulose must be characterized to assure that they are not toxic to microbial growth and ethanol production. In this study, the effects of the selected by-products which were commonly present in our acid hydrolyzate were tested.

The results indicated that furfural at concentrations as low as $1 \mathrm{mg} / \mathrm{ml}$ resulted in a significant reduction of ethanol production $(p<0.05)$ (Figure 1$)$ compared to the control $(0 \mathrm{mg} / \mathrm{ml}$ ). Ethanol production decreased from $0.273 \%$ (control) to $0.188 \%$ (1 mg/ml 
furfural), indicating that ethanol production was inhibited $31 \%$. This result was in agreement with the report by Modig [17] who showed that furfural could inhibit glycolytic enzymes at $>1 \mathrm{~g} / \mathrm{l}$. At $2 \mathrm{mg} / \mathrm{ml}$, furfural also inhibited the growth of $S$. cerevisiae. For example, with the addition of $2 \mathrm{mg} / \mathrm{ml}$ furfural, only $18 \%$ of the yeast cells survived. It is also interesting that at lower concentrations such as $1 \mathrm{mg} / \mathrm{ml}$, furfural stimulated the growth of S. cerevisiae. Specifically, cell numbers increased significantly from $8.4 \times 10^{6}$ $\mathrm{CFU} / \mathrm{ml}$ (control) to $1.17 \times 10^{7} \mathrm{CFU} / \mathrm{ml}(1 \mathrm{mg} / \mathrm{ml}$ group; $\mathrm{p}<0.05)$. We speculated that at a lower concentration furfural could be used as a growth substrate for $S$. cerevisiae.

The HMF was less inhibitory than the furfural. At $2 \mathrm{mg} / \mathrm{ml}$, it significantly inhibited the ethanol production $(p<0.05)$, while there was no significant effect on cell growth at the test concentration ( $p>0.05)$ (Figure 2). The results also showed that vanillin and vanillic acid at 2 and $0.5 \mathrm{mg} / \mathrm{ml}$, respectively, significantly inhibited ethanol production $(p<0.05)$ (Fig. 3 and 4). The inhibitory concentrations were lower in our experiment as compared to some other studies which showed that vanillin inhibited ethanol production by Saccharomyces carlsbergensis W34 at $5 \mathrm{mg} / \mathrm{ml}$ [19] and vanillic acid had no effect on ethanol fermentation by $\mathrm{S}$. cerevisiae when the concentration was $<1 \mathrm{mg} / \mathrm{ml}[1]$. It was also shown that at lower vanillic acid concentrations such as $0.5 \mathrm{mg} / \mathrm{ml}$ and 1 $\mathrm{mg} / \mathrm{ml}$, vanillic acid stimulated the growth of $S$. cerevisiae. Such stimulating effect was not detected with lower concentrations of vanillin. We speculated that lower concentrations of vanillic acid could also be used as a growth substrate for $S$. cerevisiae.

It was reported that some microelements including $\mathrm{Zn}, \mathrm{Cu}, \mathrm{Cr}$, and $\mathrm{Fe}$ might influence the stability of cell membranes, as well as the synthesis of nucleic acids and stability of the double helix of DNA while forming hydrogen bonds [5] [8] [23]. It was also reported that the addition of $200 \mathrm{mg} / \mathrm{CrCl}_{3}$ (66 ppm chromium) into the medium stimulated both the yeast growth and the ethanol production [23]. In this study, the results showed that chromium at a concentration as high as $20 \mathrm{ppm}$ had no effect on either ethanol production or microbial growth (Table 1). Chromium found in our acid hydrolyzate sample was under $20 \mathrm{ppm}$. Therefore, chromium released from the extruder reactor did not have any adverse effect on ethanol fermentation activity and cell growth or S. cerevisiae. 
Effect of the by-products mixture including chromium on ethanol production and colony-forming units was also performed in this study. Results showed that at the concentration of $1.0 \mathrm{mg} / \mathrm{ml}$, ethanol production and cell growth were significantly inhibited $(p<0.05)$ (Figure 5). Ethanol production decreased from $0.277 \%$ (control) to $0.01 \%(1.0 \mathrm{mg} / \mathrm{ml}$ by-products mixture), reflecting a $96.4 \%$ inhibition on ethanol production. At the same time, with the addition of the $1.0 \mathrm{mg} / \mathrm{ml}$ inhibitors mixture, only $23.4 \%$ cells survived the inhibition. While at lower concentrations such as $0.5 \mathrm{mg} / \mathrm{ml}$, the by-products stimulated not only the $S$. cerevisiae growth but also the ethanol production. Cells increased from $4.67 \times 10^{6} \mathrm{CFU} / \mathrm{ml}$ (control) to $1.37 \times 10^{7} \mathrm{CFU} / \mathrm{ml}(0.5$ $\mathrm{mg} / \mathrm{ml}$ by-products mixture). Ethanol production increased from $0.277 \%$ (control) to $0.294 \%(0.5 \mathrm{mg} / \mathrm{ml}$ by-products mixture), reflecting a $6.5 \%$ enhancement of ethanol production. Although the addition of $0.5 \mathrm{mg} / \mathrm{ml}$ and $1.0 \mathrm{mg} / \mathrm{ml}$ of the by-products decreased the $\mathrm{pH}$ of the medium from 6.4 to 5.0 and 4.5 , respectively, the $\mathrm{pH}$ change should not have contributed to the difference in ethanol production and cell growth. The result of a separate experiment indicated that growth and ethanol production were the same in the control medium with $\mathrm{pH} 4.5,5.0$ and 6.4 (data not shown). Therefore, the changes of ethanol production and cell growth were caused by the by-products instead of $\mathrm{pH}$. Results also showed that no effect on ethanol production and cell growth was caused by the co-existence of chromium. With the final chromium concentration of 0,8 , and $16 \mathrm{ppm}$, no change in the stimulating/inhibition pattern in ethanol production and cell growth was observed (Figure 5). This indicates that chromium did not enhance nor reduce the inhibitory effect under the test condition.

Processing method and feedstock are considered as the determining factors in ethanol production costs. Use of sawdust, a lignocellulosic biomass of no or low commercial value, could significantly reduce ethanol production costs and provide an environmentally friendly renewable energy source. However, production of various inhibitors by acid hydrolysis indicates the need for treatment before ethanol fermentation. The costs for the treatment must be reasonably low to make this route of ethanol production price-competitive. Treatment with resins is being studied in our laboratory and will be reported in the near future. 


\section{CONCLUSIONS}

Our results indicated that individual presence of furfural, HMF, vanillin, and vanillic acid at as low as $1.0,2.0,2.0$, and $0.5 \mathrm{mg} / \mathrm{ml}$, respectively, significantly inhibited ethanol production, while $\mathrm{Cr}$ did not show any inhibitory effect at concentrations up to 20 ppm. At low by-products concentrations such as $0.5 \mathrm{mg} / \mathrm{ml}$, the mixture of furfural, HMF, vanillin, and vanillic acid stimulated not only the S. cerevisiae growth but also the ethanol production, while at the concentration of $1.0 \mathrm{mg} / \mathrm{ml}$, the ethanol production and cell growth were significantly inhibited. Co-existence of chromium did neither enhance nor reduce the inhibitory/stimulating effect of the organic by-products.

\section{ACKNOWLEDGEMENTS}

This research was supported by: (1) the U.S. Department of Energy \#DE-FG02O0ER45830 with subcontract to JSU through Mississippi State University; (2) NIH-Rise \# GM 067122 (to JSU); (3) NIH-SCORE \# S06GM 08047 (to JSU); and (4) NSF-HBCUUP \# HRD-0411559. 


\section{REFERENCES}

1. Ando, S., I. Arai, K. Kiyoto, and S. Hanai. 1986. Identification of aromatic monomers in steam-exploded poplar and their influences on ethanol fermentation by Saccharomyces cerevisiae. J. Ferment. Technol. 64:567-570.

2. Atlas, R.M. 1995. Handbook of microbiological media for the examination of food. CRC Press, Boca Raton, FL. 310 pp.

3. Azhar, A.F., M.K. Bery, A.R. Colcord, R.S. Roberts, and G.V. Corbitt. 1981. Factors affecting alcohol fermentation of wood acid hydrolyzate. Biotechnol. Bioeng. Symp. 11: 293-300.

4. Benson, H.J. 1994. Microbiological applications. Wm.C. Brown communications, Inc. Dubuque, IA. 79-84 pp.

5. Beran, M., R. Stahl, and M. Beran Jr. 1995. Glycaemic activity of chromium (III)nicotinamide adenine dinucleotide phosphate complex and its presence in yeast extracts. Analyst. 120:979-981.

6. Boopathy, R., H. Bokang, and L. Daniels. 1993. Biotransformation of furfural and 5-hydroxymethyl furfural by enteric bacteria. J. Ind. Microbiol. 11:147-150.

7. Chung, I.S., and Y.Y. Lee. 1985. Ethanol fermentation of crude acid hydrolyzate of cellulose using high- level yeast inocula. Biotechnol. Bioeng. 27: 308-315.

8. Davis, C.M., and J.B. Vincent. 1997. Chromium in carbohydrate and lipid metabolism. J. Biol. Inorg. Chem. 2:675-679.

9. Delgenes, J.P., R. Moletta, and J.M. Navarro. 1996. Effect of lignocellulose degradation products on ethanol fermentation of glucose and xylose by Saccharomyces cerevisiae, Zymomonas mobilis, Pichia stipitis, and Candida shehatae. Enzyme. Microb. Technol. 19:220-225.

10. Ingram, M., D.A.A. Mossel, and P. Lange. 1955. Factors, produced in sugar-acid browning reaction, which inhibit fermentation. Chemistry and Industry. Jan.:63-64.

11. Jönsson, L.J., E. Palmqvist, N.O. Nilvebrant, and B. Hahn-Hägerdal. 1998. Detoxification of wood hydrolyzates with laccase and peroxidase from the white rot fungus Trametes versicolor. Appl. Microbiol. Biotechnol. 49:691-697.

12. Kim S., and B.E. Dale. 2002. Allocation procedure in ethanol production system from corn grain. 7 LCA (4):237-243.

13. Larsson, S., E. Palmqvist, B. Hahn-Hägerdal, C. Tengborg, K. Stenberg, G. Zacchi, and N.O. Nilvebrant. 1999. The generation of fermentation inhibitors during dilute acid hydrolysis of softwood. Enzyme Microb. Technol. 24:151-159.

14. Luo, C., D.L. Brink, and H.W. Blanch. 2002. Identification of potential fermentation inhibitors in conversion of hybrid poplar hydrolyzate to ethanol. Biomass Bioenergy 22:125-138. 
15. Martin, C., and L. Jonsson. 2002. Comparison of resistance of industrial and laboratory strains of Saccharomyces and Zygosaccharomyces to lignocellulosederived fermentation inhibitors. Enzyme. Microb. Technol. 32:386-395.

16. Martinez, A., M.E. Rodriguez, M.L. Wells, S.W. York, J.F. Preston, and L.O. Ingram. Detoxification of dilute acid hydrolyzates of lignocellulose with lime. Biotech. Prog. 17:287-293.

17. Modig, T., G. Lidén, and M.J. Taherzadeh. 2002. Inhibition effects of furfural on alcohol dehydrogenase, aldehyde dehydrogenase, and pyruvate dehydrogenase. Biochem. J. 363:769-776.

18. Palmqvist, E., J.S. Almeida, and B. Hahn-Hägerdal. 1999. Influence of furfural on anaerobic glycolytic kinetics of Saccharomyces cerevisiae in batch culture. Biotechnol. Bioeng. 62:447-454.

19. Pfeiter, P.A., G. Bonn, and O. Bobleter. 1984. Influence of biomass degradation products on the fermentation of glucose to ethanol by Saccharomyces carlsbergensis W34. Biotechnol. Lett. 6:541-546.

20. Sanchez, B., and J. Bautista. 1988. Effects of furfural and 5-hydroxymethylfurfural on the fermentation of Saccharomyces cerevisiae and biomass production from Candida guilliermondii. Enzyme Microb. Technol. 10:315-318.

21. Taherzadeh, M.J., R. Eklund, L. Gustafsson, C. Niklasson, and G. Lidén. 1997. Characterization and fermentation of dilute-acid hydrolyzates from wood. Ind. Eng. Chem. Res. 36:4659-4665.

22. Taherzadeh, M.J., L. Gustafsson, C. Niklasson, and G. Lidén. 2000. Physiological effects of 5-hydroxymethylfurfural on Saccharomyces cerevisiae. Appl. Microbiol. Biotechnol. 53:701-708.

23. Zetic, V.G., V. Stehlik-Tomas, S. Grba, L. Lutilsky, and D. Kozlek. 2001. Chromium uptake by Saccharomyces cerevisiae and isolation of glucose tolerance factor from yeast biomass. J. Biosci. 26:217-223.

24. Zhang, Y., H.-M. Hwang, M.F.T. Begonia, K.Lee, and K. Zeng. 2005. Effect of an acid hydrolyzate of southern pine softwood on the growth and fermentation ability of yeast Saccaromyces cerevisiae. J. Mississippi Aca. Sci. 50:138-143. 


\section{FIGURES}

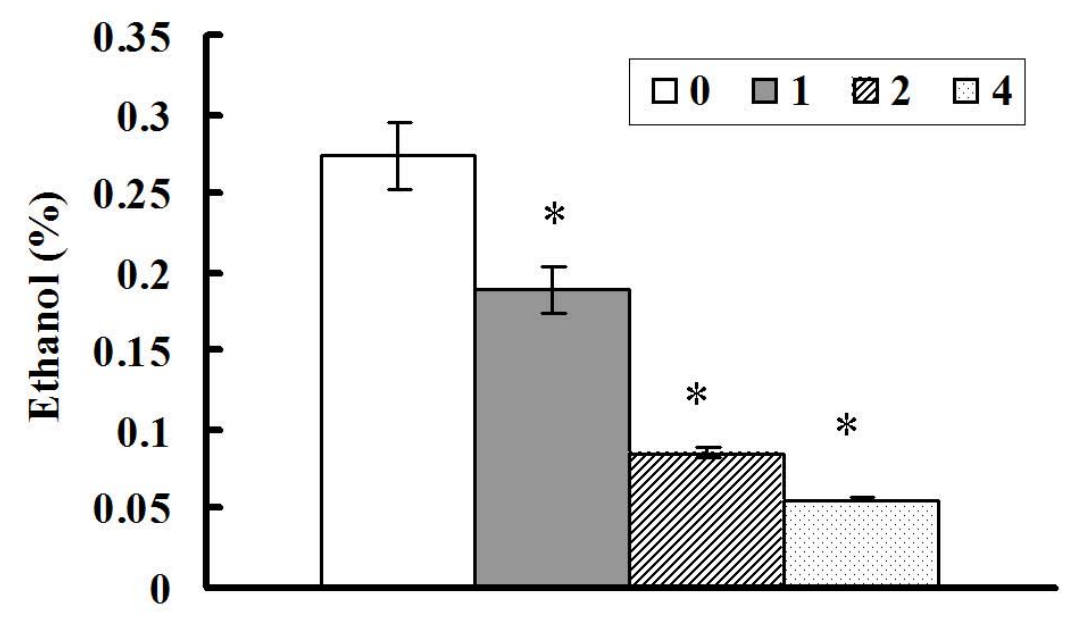

Furfural (mg/ml)

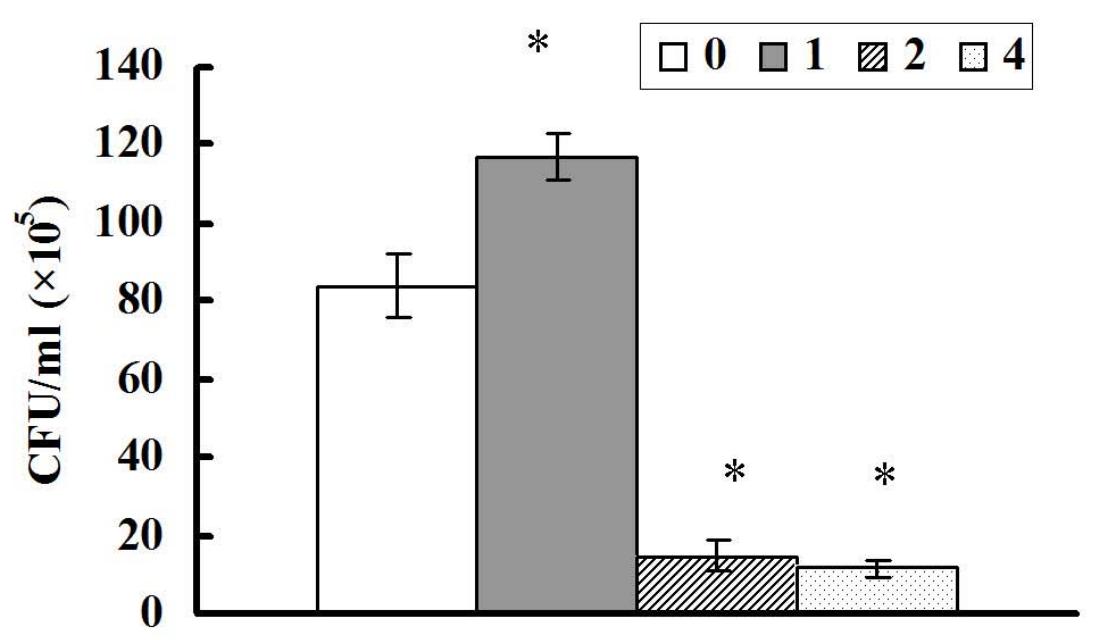

Furfural (mg/ml)

Figure 1. Effect of furfural on ethanol production and colony-forming units (CFU). S. cerevisiae grown for 2 days in fermentation medium was amended with different amounts of furfural. Samples were triplicated. Data were expressed as mean \pm SD. *denotes the significant difference from control $(0 \mathrm{mg} / \mathrm{ml}, \mathrm{p}<0.05)$. 

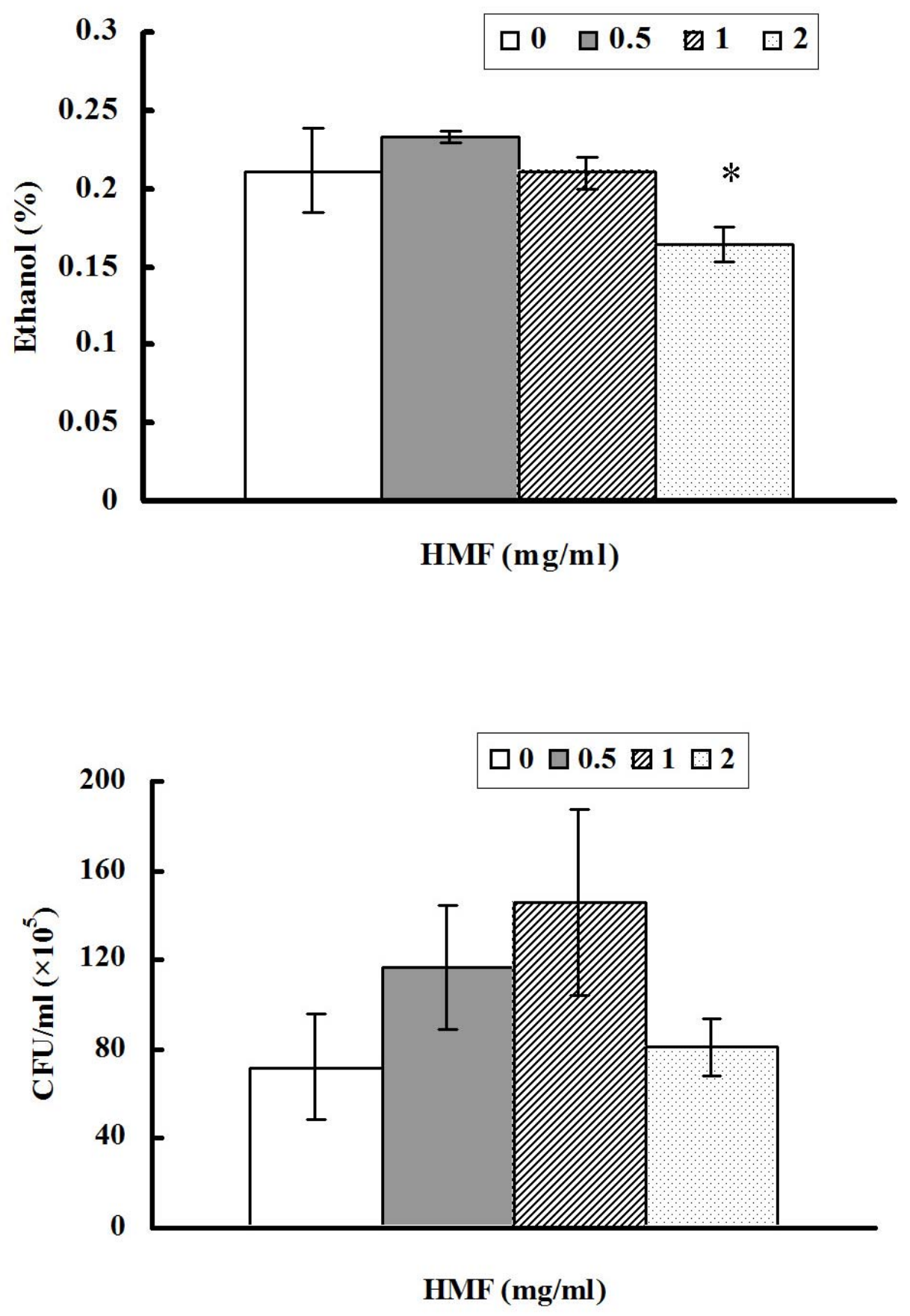

Figure 2. Effect of HMF on ethanol production and colony-forming units (CFU). S. cerevisiae grown for 2 days in fermentation medium was amended with different amounts of HMF. Samples were triplicated. Data were expressed as mean \pm SD. *denotes the significant difference from control $(0 \mathrm{mg} / \mathrm{ml}, \mathrm{p}<0.05)$. 

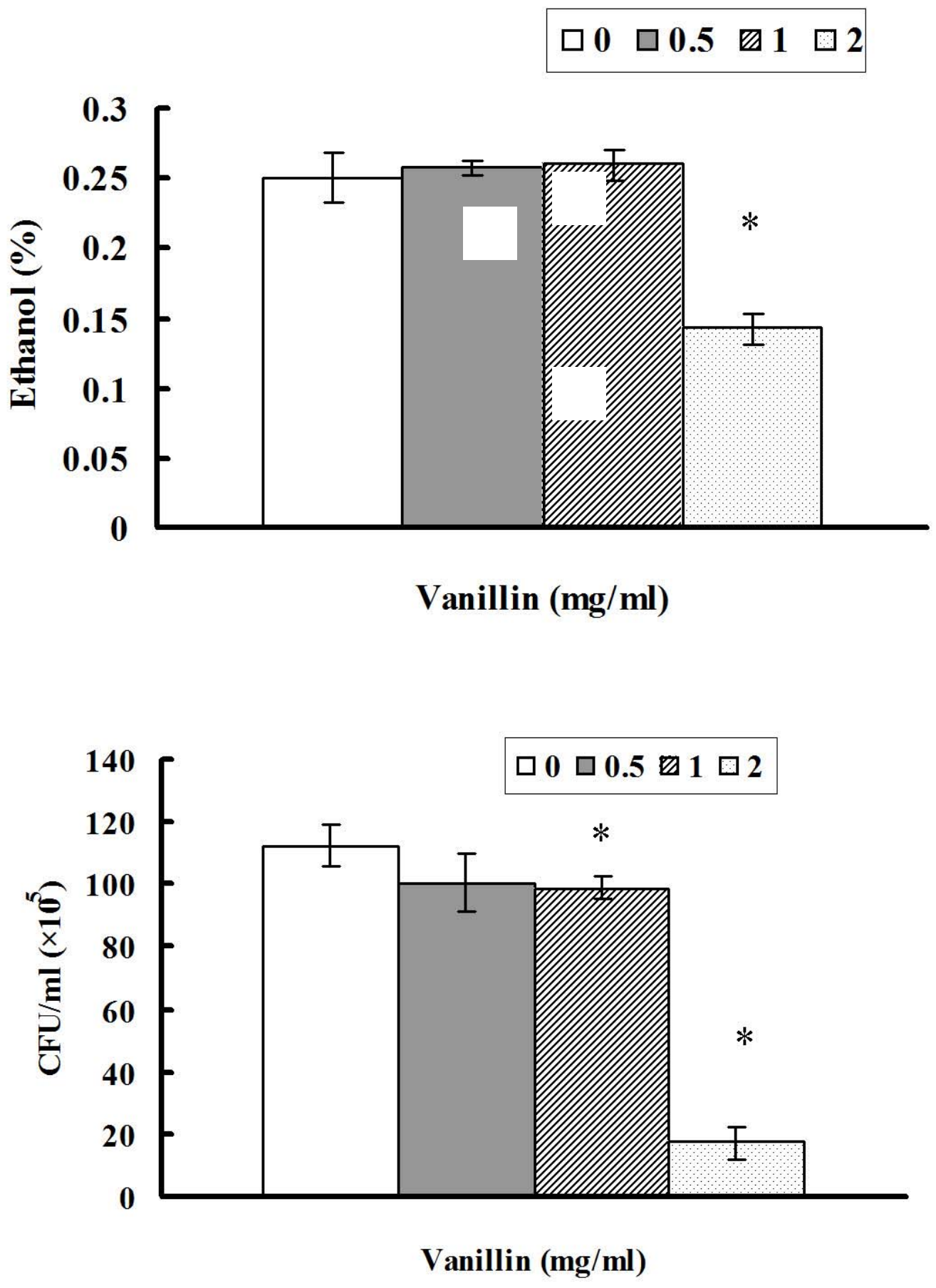

Figure 3. Effect of vanillin on ethanol production and colony-forming units (CFU). S. cerevisiae grown for 2 days in fermentation medium was amended with different amounts of vanillin. Samples were triplicated. Data were expressed as mean \pm SD. *denotes the significant difference from control $(0 \mathrm{mg} / \mathrm{ml}, \mathrm{p}<0.05)$. 

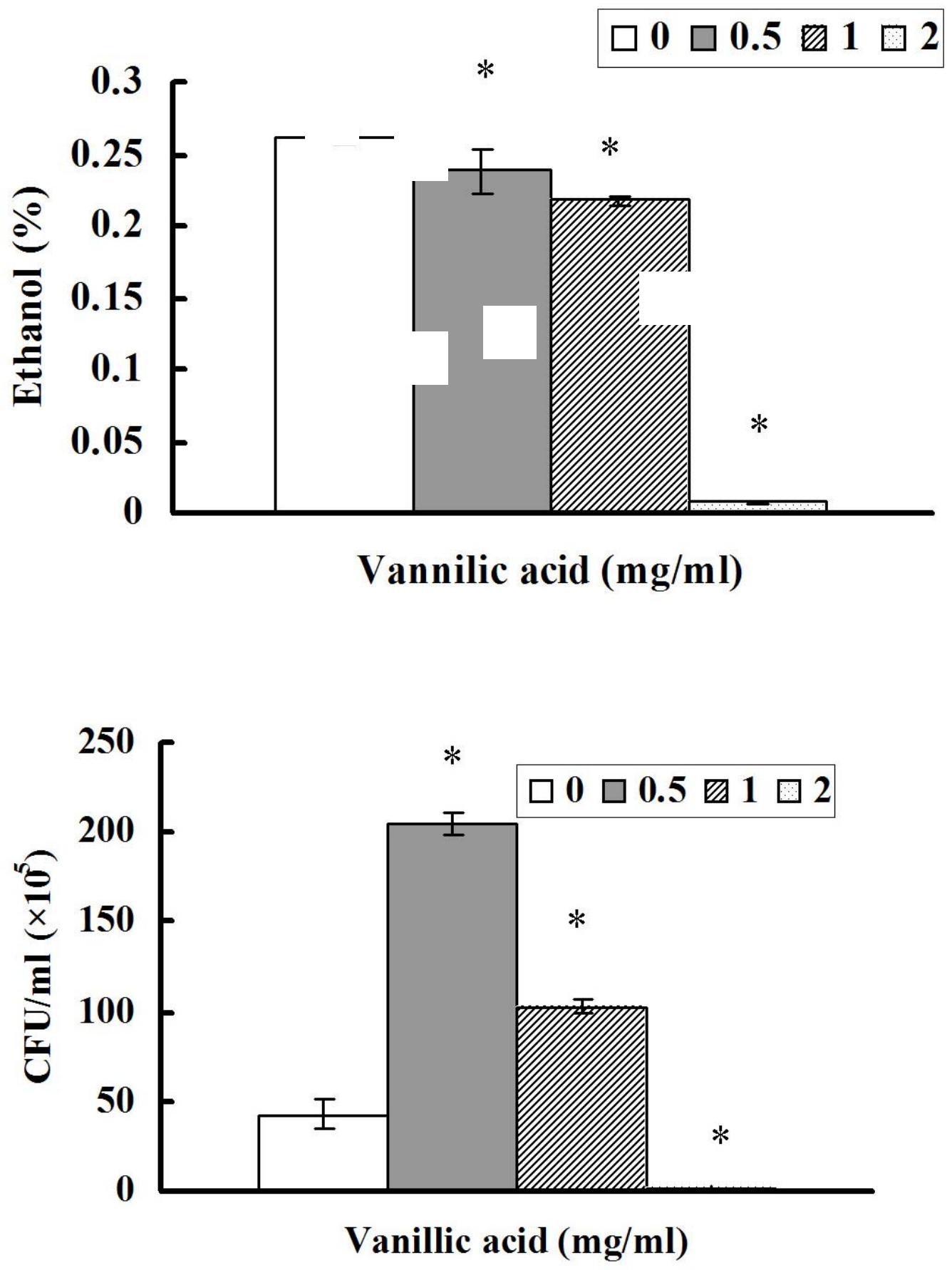

Figure 4. Effect of vanillic acid on ethanol production and colony-forming units (CFU). $S$. cerevisiae grown for 2 days in fermentation medium was amended with different amounts of vanillinic acid. Samples were triplicated. Data were expressed as mean \pm SD. *denotes the significant difference from control $(0 \mathrm{mg} / \mathrm{ml}, \mathrm{p}<0.05)$. 


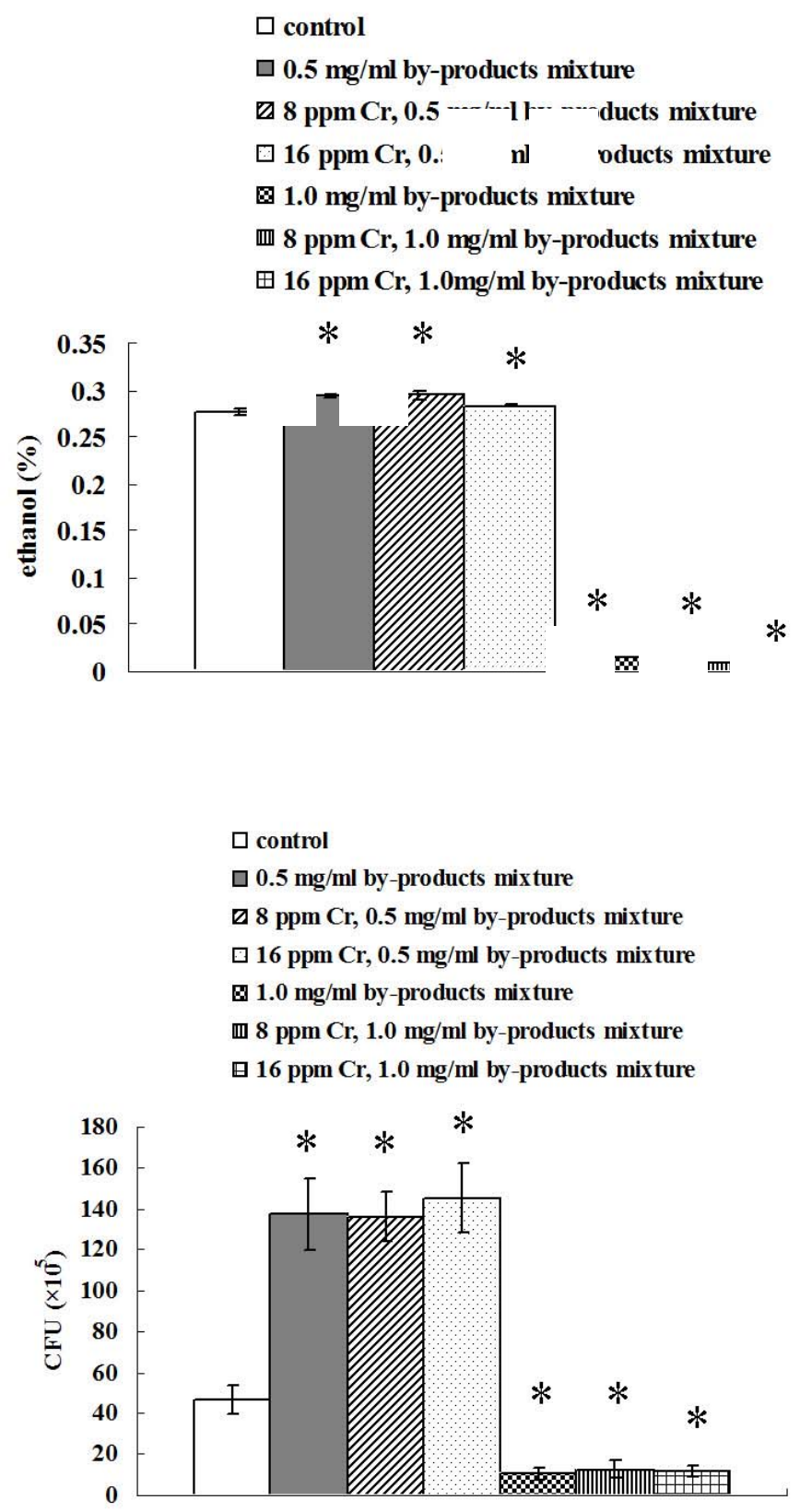

Figure 5. Effect of organic by-products mixture and organic by-products mixture plus chromium on ethanol production and colony-forming units (CFU). Fermentation medium was inoculated with S. cerevisiae. Different amounts of furfural, HMF, vanillin, and vanillic acid were added to achieve the final concentration of $0,0.5$ or $1 \mathrm{mg} / \mathrm{ml}$ each of the byproducts. Chromium nitrate was added to achieve the final concentrations of 0,8 and $16 \mathrm{ppm}$. Samples were triplicated. Data were expressed as mean \pm SD. *denotes the significant difference from control $(0 \mathrm{mg} / \mathrm{ml}, \mathrm{p}<0.05)$. 


\section{TABLE}

Table 1. Effect of chromium on ethanol production and colony-forming units (CFU) of $S$. cerevisiae grown in potato dextrose broth amended with different amounts of chromium. Samples were triplicated.

\begin{tabular}{|c|c|c|}
\hline Chromium (ppm) & CFU $\left(\times 10^{6} / \mathbf{m l}\right)$ & Ethanol (\%) \\
\hline 0 & $21.2 \pm 1.7$ & $0.36 \pm 0.006$ \\
\hline 5 & $20.9 \pm 5.7$ & $0.35 \pm 0$ \\
\hline 10 & $22.8 \pm 2.8$ & $0.35 \pm 0$ \\
\hline 20 & $26.6 \pm 4.8$ & $0.34 \pm 0$ \\
\hline
\end{tabular}




\title{
ENHANCED ETHANOL PRODUCTION FROM THE FERMENTATION OF AN ARTIFICIAL ACID HYDROLYZATE Performer: Jackson State University
}

\begin{abstract}
An alternative energy source could come from microbial fermentation of the sugars present in acid hydrolyzates derived from agricultural biomass. The fermenting microbes must be tolerant of acidic conditions and able to ferment sugars present in the hydrolyzates. Saccharomyces cerevisiae has been shown as an ideal microorganism that can ferment hexose sugars present in lignocellulose-derived hydrolyzates. The objective of this study was to assess the effect of the acid hydrolyzate of southern pine softwood sawdust on the viability and fermentation capability of $S$. cerevisiae. Acid hydrolyzate of the sawdust $(\mathrm{pH}-0.63$ ) was diluted with fermentation media at 100,300 , 400 and 800 fold. Four-day old cultures of S. cerevisiae (ATCC \#765) were then added. During the 96 -hour incubation $\left(30^{\circ} \mathrm{C}\right)$, turbidity of the medium, colony count and ethanol production were monitored. Viable number of cells and ethanol production in $300 \mathrm{X}$ and 400X- diluted groups were significantly higher than other groups in the first $24 \mathrm{hr}$ and reached the maximum after $24 \mathrm{hr}$ and $48 \mathrm{hr}$ of incubation, respectively. In a separate experiment $\mathrm{pH}$ of the fermentation medium was adjusted with $\mathrm{H}_{2} \mathrm{SO}_{4}(\mathrm{SA})$ to simulate those of the 300X and 400X diluted acid hydrolyzate (AH) groups. The results indicated that the colony counts of both $\mathrm{AH} 300$ and SA300 were significantly higher than the other groups after 48 hours, with $\mathrm{AH} 300$ being the highest among all groups. However, this pattern was not paralleled by ethanol production activity. Thus ethanol fermentability of $S$. cerevisiae exists in the extreme environment such as diluted acid hydrolyzate.
\end{abstract}

Keywords: acid hydrolyzate, ethanol, fermentation, Saccharomyces cerevisiae 


\section{INTRODUCTION}

Fossil fuels have been the major resource to meet the demanding need for energy. With the constant increase of energy consumption and the constant use of nonrenewable fuel chemicals, there is a need to find a more economical, environmentally friendly and renewable energy source [17]. Research has proven that ethanol meets these qualifications. The fact that ethanol is a byproduct of microbial fermentation makes it a renewable energy source. As an oxygenated fuel, ethanol is environmentally friendly. The major challenge is how to produce ethanol economically. Ethanol production costs have been the limiting factor in the ability of gasohol and ethanol blended fuels to compete with current gasoline cost. Processing methods and feedstock are the major factors driving ethanol cost and of the two, feedstock contributes over $50 \%$ of the total cost. Production costs can significantly be reduced with use of low cost feedstock [11] [21]. Lignocellulosic biomass, such as sawdust and wood chips, is of low commercial value but can provide inexpensive sources for ethanol production. Lignocellulosic biomass is usually composed of three primary constituents including cellulose, hemicellulose and lignin [5-7] [14]. Although wood residues such as sawdust and woodchips are readily available, the sugars suitable for fermentation processes and alcohol yield are only found in cellulose and hemicellulose constituents. Cellulose contains hexose sugars (mainly glucose) and hemicellulose contains pentose sugars (mainly xylose). In order to obtain the sugars needed for fermentation pretreatment of lignocellulosic biomass is necessary.

The most widely used pre-treatment method is acid hydrolysis. Concentrated acid hydrolysis, one of the acid hydrolysis methods, involves use of concentrated acid (sulfuric acid, $\mathrm{H}_{2} \mathrm{SO}_{4}$ or hydrochloric acid, $\mathrm{HCl}$ ) in the presence of high temperature $\left(190^{\circ} \mathrm{C}-215^{\circ} \mathrm{C}\right)$ to split cellulose and hemicellulose into simple sugars [16]. The concentration of sugars tends to be higher if the lignocellulosic biomass is treated with extreme acidity and high temperature; however, the products of acid hydrolysis (acid hydrolyzate) become very acidic and may contain a variety of inhibitory compounds such as furfural, 5-hydroxymethylfurfural (5-HMF), acetate and other phenolic compounds [10[ [13] [8]. In order to maximize recycling of the acid present in the 
hydrolyzate, it is desirable to use acid-tolerant microorganisms to conduct ethanol fermentation process. Saccharomyces cerevisiae has been shown as the yeast that can best ferment hexose sugars present in lignocellulose-derived hydrolyzates due to its ethanol-producing capacity and high inhibitor tolerance [8] [12]. Even though $S$. cerevisiae grows optimally around pH 5 (ATCC 2001 Product Information Sheet for ATCC 765), one study showed that the yeast is able to grow at a pH as low as 2.5 [19]. The objective of this study was to assess the effect of the acid hydrolyzate of a southern pine softwood sawdust on the viability and potential fermentation capability of $S$. cerevisiae. The ultimate goal is to develop a specific acid hydrolysis procedure to minimize the amount of inhibitory hydrolyzate constituent for $S$. cerevisiae and to maximize ethanol fermentation within the most tolerable acidity level. Consequently, the cost for recycling the sulfuric acid and the associated impact on environmental health would be reduced.

\section{MATERIALS AND METHODS}

A 4 day old culture of Saccharomyces cerevisiae (ATCC\#765) was used in the experiment. The 4 day old culture was prepared by rehydrating the freeze-dried culture in $5 \mathrm{~mL}$ sterile distilled water overnight and then transferred to potato dextrose broth (24 g/L, pH 4.2) for enrichment (ATCC 2001 Product Information Sheet for ATCC 765). According to chemical analysis with the method developed by National Renewable Energy Laboratory (NREL) at Golden, Colorado [15], pre-treatment of southern pine softwood sawdust by acid hydrolysis $\left(\mathrm{H}_{2} \mathrm{SO}_{4}\right)$ yielded an acid hydrolyzate $(\mathrm{pH}-0.63)$ that contained the following sugars and other organic compounds: $26.65 \mathrm{mg} / \mathrm{mL}$ glucose, $6.7 \mathrm{mg} / \mathrm{mL}$ mannose, $4.48 \mathrm{mg} / \mathrm{mL}$ xylose, 2.78 $\mathrm{mg} / \mathrm{mL}$ galactose, $1.4 \mathrm{mg} / \mathrm{mL}$ arabinose, and $1.64 \mathrm{mg} / \mathrm{mL}$ acetic acid. Xylitol, lactic acid, glycerol, ethanol, HMF and furfurals were not detected. In Pyrex flasks containing $90 \mathrm{~mL}$ fermentation media (Atlas 1995) (1\% trypticase, $0.1 \%$ beef extract, $0.5 \%$ dextrose and $0.5 \% \mathrm{NaCl}, \mathrm{pH} 6.4$ ), $10 \mathrm{~mL}$ of $\mathrm{S}$. cerevisiae (in PDB, cell density $15 \times 10^{5} / \mathrm{mL}$ ) was added. A different amount of acid hydrolyzate was added to the flasks to reach a final concentration of $100,300,400$ and 800 fold dilution of the acid hydrolyzate. The flask containing only fermentation media and the cells served as the control. Fermentation flasks were incubated at $30^{\circ} \mathrm{C}$ and sampled at time points 0,24 , 
48, 72 and 96 hours. The following assays were conducted: (1) turbidity of the growth medium [4]; (2) colony count on potato dextrose agar plate [4]; and (3) ethanol production.

Briefly, turbidity (Klett unit) was measured with the photoelectric colorimeter at spectral range 520-580 nm (Tube Model 800-3, Scienceware, Bel-Art Products, Pequannock, NJ). Concurrently $\mathrm{pH}$ and colony count $(\mathrm{CFU} / \mathrm{mL})$ on potato dextrose agar were recorded. The samples were then filtered through a $0.2 \mu \mathrm{m}$ HT Tufftyn ${ }^{\circledR}$ membrane filter (Acrodisc syringe filter, Pall Corp., Ann Arbor, MI) and alcohol production was measured by gas chromatograph (Shimadzu GC-14A, Shimadzu Corp., Kyoto, Japan) with a flame ionization detector (FID). Samples were separated on a $30 \mathrm{~m} \times 0.53 \mathrm{~mm}$ fused-silica capillary column with $1.0 \mu \mathrm{m}$ thickness film. The oven temperature was programmed as follows: $40^{\circ} \mathrm{C}(0.3 \mathrm{~min}), 40-85^{\circ} \mathrm{C}\left(20^{\circ} \mathrm{C} / \mathrm{min}\right)$ and $85-$ $125^{\circ} \mathrm{C}\left(40^{\circ} \mathrm{C} / \mathrm{min}\right)$. Injector temperature was $230^{\circ} \mathrm{C}$. Injection volume was $0.1 \mathrm{~mL}$.

In a separate experiment the $\mathrm{pH}$ values of the 300- and 400-fold diluted acid hydrolyzate were simulated by adding 0.6 and $1 \mathrm{~mL}$ of $2 \mathrm{~N} \mathrm{H}_{2} \mathrm{SO}_{4}$ to the Pyrex flasks containing the mixture of $90 \mathrm{~mL}$ fermentation media and $10 \mathrm{~mL}$ S. cerevisiae, respectively. Various sugars present in the diluted acid hydrolyzate were added at the corresponding concentrations. The same assays were conducted following the aforementioned procedures. Difference in the experimental data between the treatment groups or between a treatment group and its relevant control group is determined with either SAS or Student's t-test $(p<0.05)$.

\section{RESULTS AND DISCUSSION}

One of the requisites for efficient growth and ethanol production is the tolerance of the fermenting microbe ( $S$. cerevisiae) to the wood hydrolyzate. However, our previous experiments showed that the undiluted wood hydrolyzate $(\mathrm{pH}-0.63)$ inhibited the growth and the fermentation ability of $S$. cerevisiae in the media containing undiluted hydrolyzate (data not shown). Due to the toxicity of the undiluted acid hydrolyzate, the hydrolyzate was diluted with fermentation medium up to 800 fold. After 1, 2, 3, and 4 days of incubation, the mean $\mathrm{pH}$, growth in terms of Klett unit and number of colony forming units per $\mathrm{mL}$ of medium, and percent ethanol production for each treatment (or 
dilution) and for the control were determined. As evidenced by the $\mathrm{pH}$ data of the control group (yeast in fermentation medium without acid hydrolyzate), fermentation process decreased $\mathrm{pH}$ from the initial value of 5.8 to 4.6 and stabilized it around 4.6 within the 4day incubation period. Consistent $\mathrm{pH}$ values were also observed for the other groups, with the $100 \times$ group maintaining the lowest $\mathrm{pH}$ number (Figure 1a). Concurrent measurements of viability, biomass and ethanol production levels are indicated in units of Klett turbidity unit (Figure 1b), cfu $\times 10^{5} / \mathrm{mL}$ (Figure 1c) and \% ethanol production (Figure 1d), respectively. All numbers of the 100x group remained low throughout the incubation period, indicating that cellular growth and fermentation activity of the yeast culture were inhibited by the 100 X-diluted acid hydrolyzate ( $\mathrm{pH} 1.8$ - 2.0). At extracellular $\mathrm{pH}$ values below the pKa value of acetic acid (4.75), the undissociated acid can diffuse through the cellular membrane and dissociate intracellularly. ATP consumption rate will increase and hence the growth rate will decrease when the proton pump process is activated to maintain a constant intracellular $\mathrm{pH}$ [18].

Viable number of cells (expressed as colony forming units $\times 105 / \mathrm{mL}$ of medium) and ethanol production of the test yeast in $300 \times$ and $400 X$ - diluted groups were significantly higher than other groups in the first $24 \mathrm{hr}$ and reached the maximum after $24 \mathrm{hr}$ and $48 \mathrm{hr}$ of incubation, respectively (Figures $1 \mathrm{c}$ and $1 \mathrm{~d}$ ). Figure $1 \mathrm{~b}$ indicates that Klett units of the 300X and 400X-diluted groups are the highest among all groups; however, the turbidity units of all groups continued to increase during the incubation period with the exception of 100X group. Although clinically Klett colorimetry has been extensively used to monitor the growth curve of microorganisms, the turbidity scale does not necessarily correlate with active or living biomass of the test microorganisms, except during the exponential growth phase. The experiment was repeated several times and the yeast populations consistently grew and fermented better in the 300-400X groups. Yeasts exhibit a considerable tolerance to extremes of $\mathrm{pH}$. They are capable of maintaining active fermentation in glucose solution between a $\mathrm{pH}$ range of 2.4 to 7.4 [9].

For optimum production of ethanol, routine practice dictates that the $\mathrm{pH}$ of the fermenting medium is maintained within the range of 4.0 to 6.0. The explanation to the test yeast's ability to grow and ferment in 300X, 400X and 800X-diluted groups (pH 3.04.2; Figures $1 \mathrm{a}$ and $1 \mathrm{~d}$ ) is that the intracellular $\mathrm{pH}$ of the yeast cells remains quite 
constant at about $\mathrm{pH} 5.8$, regardless of the wide $\mathrm{pH}$ variations in the surrounding environment. The enzymes involved in fermentation thus operate in an optimum $\mathrm{pH}$ environment within the yeast cells. Addition of acetate to a growth medium during anaerobic continuous and batch cultivations was shown to lower the biomass yield while enhancing the ethanol yield by S. cerevisiae [20] [18]. The increase in ethanol yield was speculated to occur due to the uncoupling effect of acetic acid on ATP reductions, provided that byproduct formation does not increase during the sugar to ethanol conversion process [19]. Our results partially contradict those findings. In the case of 300X and 400X-diluted groups in our batch study, both biomass and ethanol production rates were significantly enhanced in 24 hours (SAS; $p<0.05$ ). The net effect of acetate on biomass yield or ethanol formation rate may vary, depending on the growth conditions or whether the specific growth rate is affected [18]. Further studies are needed to confirm the effect of acetic acid on biomass yield and ethanol production.

In a separate experiment the $\mathrm{pH}$ of the fermentation medium was adjusted with $\mathrm{H}_{2} \mathrm{SO}_{4}(\mathrm{SA})$ to simulate those of the $300 \mathrm{X}$ and $400 \mathrm{X}$ diluted acid hydrolyzate (AH) groups (Figure 2a). Colony count and ethanol production of the SA and $\mathrm{AH}$ groups were compared (Figures $2 \mathrm{~b}$ and 2c). Colony counts of both $\mathrm{AH} 300$ and SA300 were significantly higher than the other groups at 48 hours (Figure 2b), with AH300 being the highest among all groups ( $p<0.05$; Student's t-test). However, this pattern was not paralleled by the ethanol production (Figure 2c) in which AH300 and SA300 were indistinguishable from other treatments in the first $48 \mathrm{hr}$. Except for acetic acid, all sugars in the diluted acid hydrolyzate were added to the 300X and 400X SA groups. Since various sugars and acetic acid were the only prevalent organic species detected in the acid hydrolyzate, the significant increase in the number of viable cells of the $300 \mathrm{X}$ groups could not be attributed to the presence of acetic acid. Apparently more study is needed to understand the complicated growth energetics of $S$. cerevisiae in the presence of acid hydrolyzate. Results of this study indicate that the ethanol fermentation capability of the yeast exists in the extreme environment such as diluted acid hydrolyzate. However, low sugar concentrations in the diluted acid hydrolyzate could not support the test yeast's fermentation activity. Other pre-treatment methods, such as overliming and ion exchange, were reported to result in higher ethanol 
productivity [13]. Comparison studies on the effects of the aforementioned pre-treatment methods on sugar concentrations and ethanol production will be conducted in the near future.

\section{ACKNOWLEDGEMENTS}

This research was supported by: (1) the U.S. Department of Energy \#DE-FG02O0ER45830 with subcontract to JSU through Mississippi State University and (2) U.S. Department of the Army \# DAAD 19-01-1-0733 (to JSU). We thank Dr. Roger Hester of the Department of Polymer Science, University of Southern Mississippi for providing acid hydrolyzate and Dr. Bonnie R. Hames of the National Renewable Energy Lab, Golden, Colorado for chemical analysis of acid hydrolyzate. We also thank Ms. Ana L. Balarezo for providing technical assistance in this study. 


\section{REFERENCES}

1. American Type Culture Collection. 2001. Product Information Sheet for ATCC ${ }^{\circledR} 765$, P.O. Box 1549, Manassas, VA 20108.

2. Atlas, R.M. 1995. Alphabetical listing of media. 9-515. in L.C. Parks LC, ed.

3. Handbook of media for environmental microbiology. CRC Press, Boca Raton, FL. 365 pp.

4. Benson, H.J. 1994. Microbiological applications. Wm. C. Brown Communications, Inc, Dubuque, IA. 79-84 pp.

5. Boopathy, R. 1998. Biological treatment of swine waste using anaerobic baffled reactors. Bioresor Technol 64:1-6.

6. Cheung, S.W. and Anderson, B.C. 1997. Laboratory investigation of ethanol production from municipal primary wastewater. Bioresor Technol 59:81-96.

7. Dewes, T. and Hünsche, E. 1998. Composition and microbial degradability in the soil of farmyard manure from ecologically-managed farms. Biol Agric Hortic 16:251268.

8. Hahn-Hägerdal, B.; Linden, T.; Senac, T.; and Skoog, K. 1991. Ethanolic fermentation of pentoses in lignocellulosic hydrolysates. Appl Biochem Biotechnol 28/29: 131-144.

9. Http://www.home.earthlink.net/ ggda/factors effecting fermentation.htm. August, 2004

10. Luo, C.; Brink, D.L., and Blanch, H.W. 2002. Identification of potential fermentation inhibitors in conversion of hybrid polar hydrolyzate to ethanol. Biomass Bioenergy 22: $125-138$.

11. Mani, S., Tabil, L.G.; and Opoku, A. 2002. Ethanol from agricultural crop residues. An overview. American Society of Agricultural Engineers North Central Intersectional Conference. Paper No. MBSK 02-217.

12. Olsson L., and Hahn-Hägerdal, B. 1993. Fermentative performance of bacteria and yeasts in lignocellulosic hydrolysates. Process Biochem 28: 249-257.

13. Palmqvist, E., and Hahn-Hägerdal, B.2000. Fermentation of lignocellulosic hydrolysates. I: inhibition and detoxification. Bioresor Technol 74: 17-24.

14. Reshamwala, S.; Shawky, B.T., and Dalw, B.E.. 1995. Ethanol production from enzymatic hydrolysates of AFEX-treated coastal Bermuda grass and switchgrass. Appl Biochem Biotechnol 51/52: 43-45.

15. Ruiz, R., and Ehrman, T. 1996. Determination of carbohydrates in biomass by high performance liquid chromatography. Laboratory Analytical Procedure LAP-002. NREL, Golden, Colorado.

16. Springfield, R.M., and Hester, R.D. 1999. Continuous ion-exclusion chromatography system for acid/sugar separation. Separation Sci Tech 34 (6\&7): 1217-1241. 
17. Sun, Y., and Cheng, J. 2002. Hydrolysis of lignocellulosic materials for ethanol production: a review. Bioresor Technol 83: 1-11.

18. Taherzadeh, M.J.; Lidén, G.; Gustafsson, L., and Niklasson, C. 1997a. The effects of panthenate deficiency and acetate addition on anaerobic batch fermentation of glucose by Saccharomyces cerevisiae. Appl Microbiolo Biotechnol 46: 176-182.

19. Taherzadeh, M.J.; Niklasson, C., and Lidén, G. 1997b. Acetic acid-friend or foe in anaerobic batch conversion of glucose to ethanol by Saccharomyces cerevisiae. Chem Eng Sci 52: 2653-2659.

20. Verduyn, C.; Postma, E.; Scheffers, W.A., and vanDijken, J.P. 1990. Physiology of Saccharomyces cerevisiae in anaerobic glucose-limited chemostat cultures. J Gen Microbiol 136: 305-319.

21.Zerbe, J.I. 1992. Liquid fuels from wood - ethanol, methanol, and diesel. Wood Resource Review 3(4). Effect of an Acid Hydrolyzate of Southern Pine Softwood on the Growth and Fermentation Ability of Yeast Saccharomyces cerevisiae

22.Zhang, Yi; Hwang, Huey-Min; Begonia, Maria F.T.; Lee, Ken, and Zeng, Kui Department of Biology, Jackson State University, 1400 Lynch Street, Jackson, MS 39217 


\section{FIGURES}
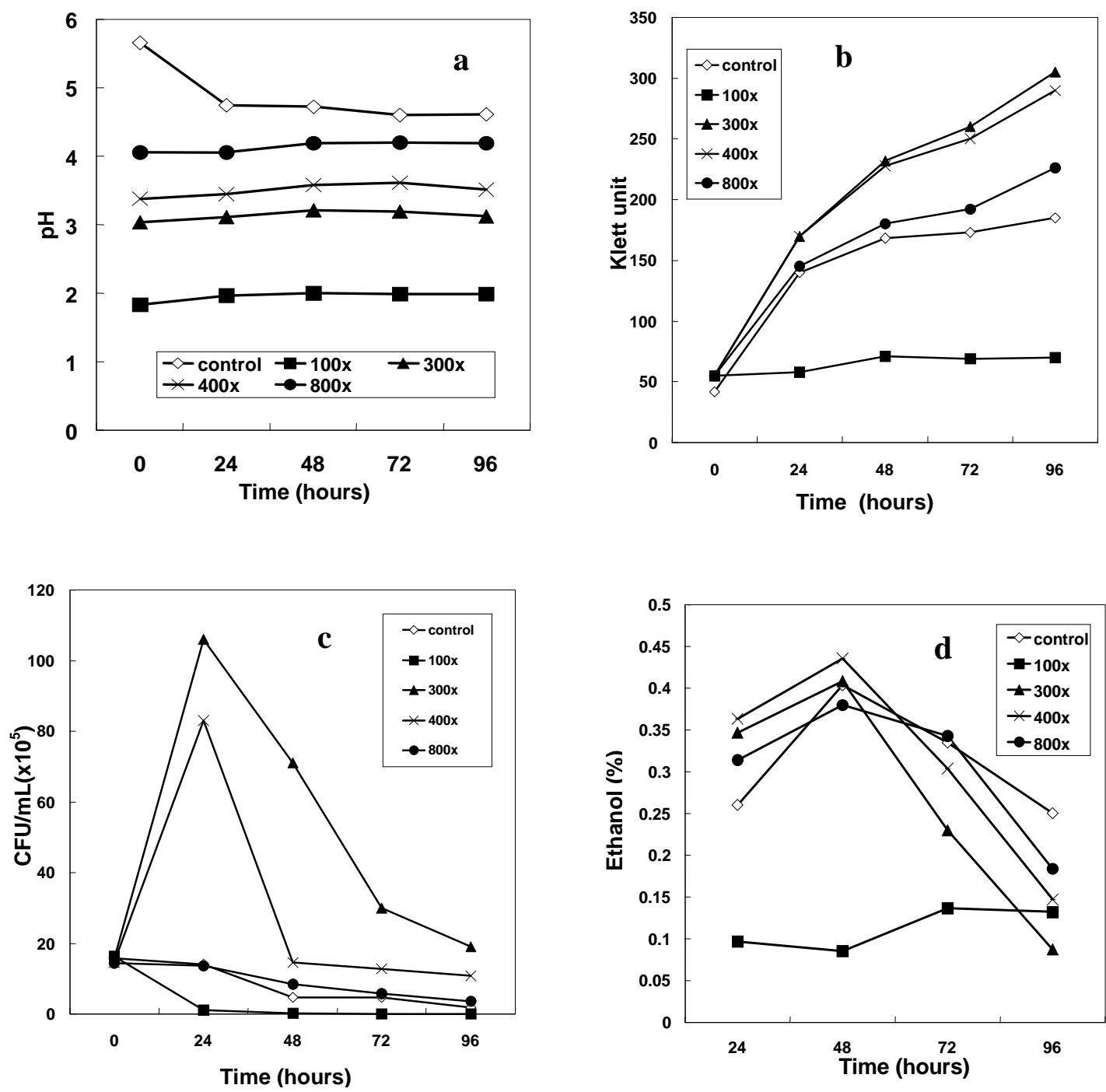

Figure 1. pH (a), Klett unit (b), cfu (c), and ethanol production (d) of Saccharomyces cerevisiae culture ( $\mathrm{pH} 4.06)$ mixed with diluted acid hydrolyzate at the ratio of $1: 10$. All samples were run in triplicate. Variations were $<10 \%$ among the replicate. 

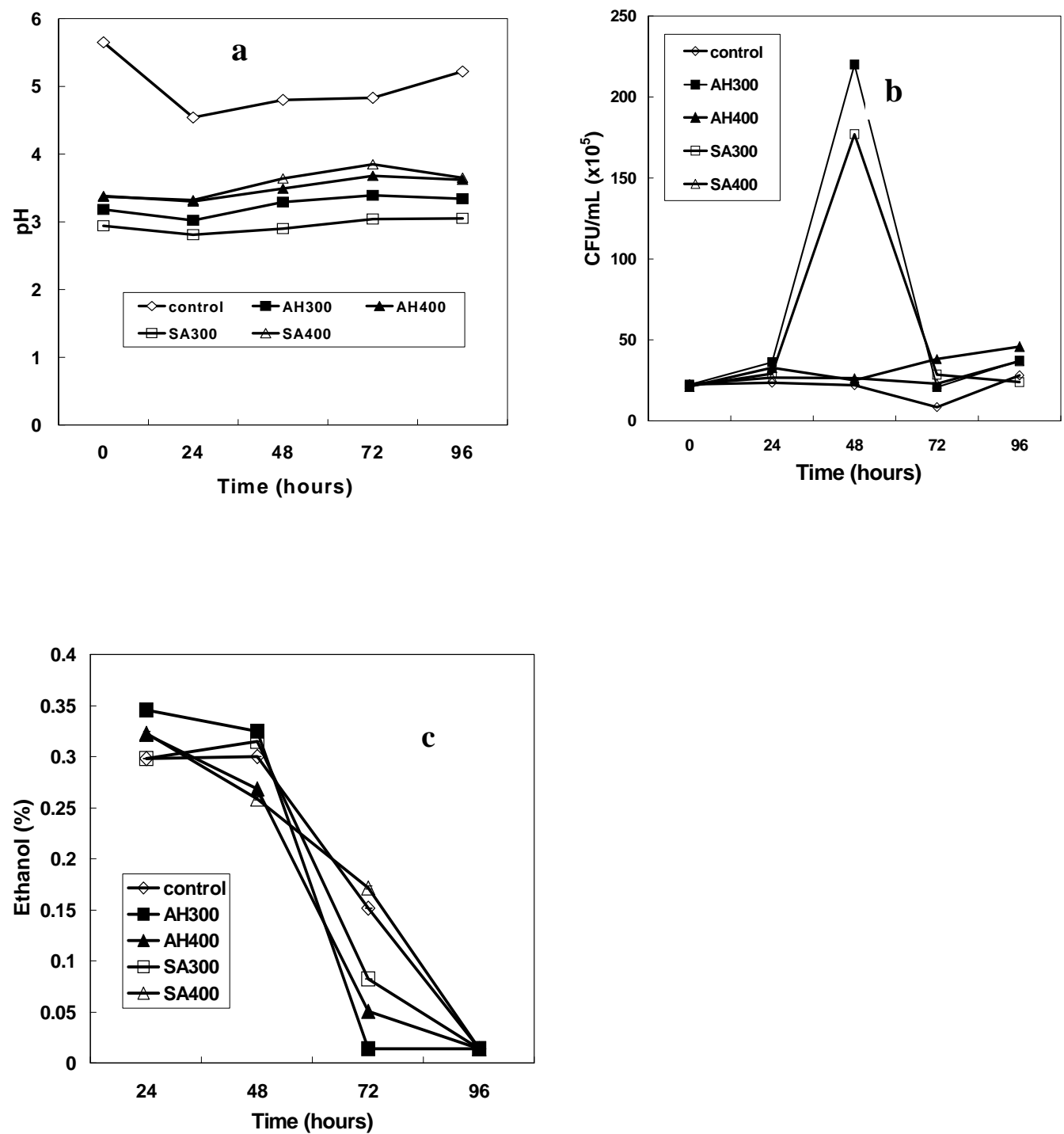

Figure 2. $\mathrm{pH}(\mathrm{a})$, cfu (b) and ethanol production (c) of samples. Saccharomyces cerevisiae ( $\mathrm{pH} 4.06)$ culture was added to 300 and 400 fold diluted $\mathrm{AH}$ (AH300 and $\mathrm{AH} 400$ ) or fermentation media with $\mathrm{pH}$ adjusted by $\mathrm{H}_{2} \mathrm{SO}_{4}$ to its corresponding diluted $A H$ (SA300 and SA400). All samples were run in triplicates. Variations were $<10 \%$ among the replicate. 


\section{ENHANCING ETHANOL FERMENTABILITY OF AN ARTIFICIAL ACID HYDROLYZATE WITH TREATMENT OF ANION EXCHANGE RESINS Performer: Jackson State University}

\section{INTRODUCTION}

With the constant increase of energy consumption and the constant use of nonrenewable fuel chemicals, there is a need to find a more economical, environmentally friendly and renewable energy source. Research has proven that ethanol meets these criteria [1]. Lignocellulosic biomass, such as sawdust and wood chips, is of low commercial value but can provide inexpensive sugar sources for ethanol production. The conversion of lignocellulosic biomass to simple sugars, which will be used for microbial fermentation, can be achieved by acid hydrolysis. However, the acid hydrolyzate (product of hydrolysis) usually is very acidic $(\mathrm{pH}<1)$ and contains not only the sugars but also furan and phenolic compounds as well as many other inhibitors of fermentation [2] [3]. The furan compounds found in wood hydrolyzates include 5hydroxymethylfurfural (HMF) and 2-furaldehyde (furfural) which are produced by high temperature degradation of hexose and pentose sugars, respectively. The phenolic compounds, mainly vanillin and vanillic acid, are formed by the partial breakdown of lignin [4] [5].

The effect of individual inhibitor on microbial fermentation has been studied. Furfural was considered to be an inhibitor in "batch" cultivation [6]. The presence of furfural at $1 \mathrm{mg} \mathrm{mL}^{-1}$ was reported to decrease the $\mathrm{CO}_{2}$ evolution [6], cell multiplication [7] and total viable cell [8] of the test microorganisms in the early phase of fermentation. HMF is an inhibitor of Saccharomyces cerevisiae growth, but not as strong as furfural [6]. An early study showed that HMF at concentration of $1 \mathrm{mg} \mathrm{mL}^{-1}$ had an inhibitory effect on baker's yeast [9]. A more recent study reported that only at higher concentration ( $\left.2 \mathrm{mg} \mathrm{mL}^{-1}\right)$, it decreased bacteria biomass yield by $23 \%$ [10]. Vanillin was one of the strongest inhibitors among the hydrolysis by-products [11]. It significantly inhibited ethanol production by Saccharomyces carlsbergensis $\mathrm{W} 34$ at $5 \mathrm{mg} \mathrm{mL}^{-1}$ [12]. When concentration was $<1 \mathrm{mg} \mathrm{mL}^{-1}$, vanillic acid had no effect on ethanol fermentation by S. cerevisiae [13]. 
Nevertheless, the aforementioned inhibitor compounds may coexist in acid hydrolyzates and act synergistically in inhibiting fermentation activity [3]. Therefore, pretreatment of the acidic and toxic hydrolyzate must be conducted before proceeding to the fermentation step. Overliming with $\mathrm{Ca}(\mathrm{OH})_{2}$ or $\mathrm{NaOH}$ is one of the methods commonly used for neutralizing and detoxifying acid hydrolyzate. However, significant loss of sugars, limited capability of removing organic inhibitors (e.g., phenolic compounds) [5] and production of fermentation-inhibiting salts were considered as the weakness of this method.

Anion exchange resin is a polymer with electrically charged sites at which one ion may replace another. Ion exchange technique was used for separating acids/sugars and treating hydrolyzate for ethanol fermentation [3] [14]. Dowex M43 and Dowex monosphere 66 are weak base anion exchange resins which act like an acid absorber. According to the specification information from the vendor, their exchange capacities are 1.55 and $1.6 \mathrm{eq} \mathrm{L}^{-1}$ respectively. These resins are widely used in industries for corrosion control, sweetener applications and water demineralization applications. They can be regenerated for additional operating cycles.

The objective of this research was to study the efficiency of neutralization and detoxification of an artificial inhibitor cocktail solution by using two different resins, Dowex M43 and Dowex monosphere 66, and compare their detoxification effectiveness.

\section{MATERIALS AND METHODS}

\section{Chemicals}

Chemicals including 2-furaldehyde (furfural), 5-hydroxymethylfurfural (HMF), vanillin, vanillic acid, water (HPLC grade) and the test resins (Dowex M43 and Dowex monosphere 66) were purchased from Sigma (St. Louis, MO). Sugars including glucose, mannose, xylose, galactose and arabinose were purchased from Fisher Scientific (Houston, TX).

\section{Detoxification of Inhibitors}

To assess the effectiveness of two resins in removing the inhibitors, $10 \mathrm{~mL}$ of inhibitor cocktail containing $2 \mathrm{mg} \mathrm{mL}^{-1}$ each of furfural, $\mathrm{HMF}$, vanillin and vanillic acid ( $\mathrm{pH}$ 0.63) was added to a beaker containing $10 \mathrm{~g}$ (wet weight) pre-soaked resin. The 
mixture was stirred consistently and sampled at $2.5,5,10$, and 20 min for analysis of inhibitor concentrations. The $\mathrm{pH}$ was monitored during the experimental period.

\section{Effectiveness of Resins in Detoxification of the Inhibitor Cocktail}

To determine the effectiveness of the resins in detoxification the inhibitor cocktail, $10 \mathrm{~mL}$ fermentation medium ( $1 \%$ trypticase, $0.1 \%$ beef extract, $0.5 \%$ dextrose, and $0.5 \%$ sodium chloride) containing $2 \mathrm{mg} \mathrm{mL}^{-1}$ each of furfural, HMF, vanillin and vanillic acid ( $\mathrm{pH}$ 0.63) was added to a beaker containing $10 \mathrm{~g}$ (wet weight) pre-soaked Dowex M43 or Dowex monosphere 66 resins. The mixture was stirred consistently and pH was monitored. When the $\mathrm{pH}$ value reached 3.5 or slightly higher values, $10 \mathrm{~mL}$ samples were taken and filtered through $0.2 \mu \mathrm{m}$ filter (syringe filter, Daigger, Vernon Hills, IL). Ten $\mathrm{mL}$ untreated fermentation medium was used as control. One $\mathrm{mL}\left(10^{6} \mathrm{~mL}^{-1}\right)$ of $\mathrm{S}$. cerevisiae was added. The ethanol carried over with the inoculum was less than $0.01 \%$. According to our previous study, ethanol production peaks between 18-24 $\mathrm{h}$, ethanol production and viable cell number were determined after 18 hours of incubation at $30^{\circ} \mathrm{C}$. The viable cell number count was carried out on potato dextrose agar plates [15].

\section{Sugar Recovery}

Two sugar cocktail solutions, which were comparable to the sugar concentrations in two different acid hydrolyzates used in our study, were made [16]. Cocktail \#1 contains lower total sugar concentration $\left(9 \mathrm{mg} \mathrm{mL}^{-1}\right.$ ), including $2.5 \mathrm{mg} \mathrm{mL}^{-1}$ glucose, 1 $\mathrm{mg} \mathrm{mL}^{-1}$ xylose, $1.5 \mathrm{mg} \mathrm{mL}^{-1}$ galactose and $4 \mathrm{mg} \mathrm{mL}^{-1}$ mannose ( $\mathrm{pH}$ 0.65). Cocktail \#2 contains higher total sugar concentration ( $26 \mathrm{mg} \mathrm{mL}^{-1}$ ), including $15 \mathrm{mg} \mathrm{mL}^{-1}$ glucose, 3 $\mathrm{mg} \mathrm{mL}^{-1}$ xylose, $2 \mathrm{mg} \mathrm{mL}^{-1}$ galactose, $1 \mathrm{mg} \mathrm{mL}^{-1}$ arabinose and $5 \mathrm{mg} \mathrm{mL}^{-1}$ mannose ( $\mathrm{pH}$ 0.65). Ten $\mathrm{mL}$ sugar solution was added to $15 \mathrm{~g}$ (wet weight) pre-soaked resin and stirred consistently for $10 \mathrm{~min}$. When the $\mathrm{pH}$ value reached 5.0, samples were removed for analysis of sugar concentration.

\section{Equipment and Analytical Methods}

Ethanol concentration was determined by gas chromatography (Shimadzu GC14A, Shimadzu Corp., Kyoto, Japan) equipped with a flame ionization detector (FID). The oven temperature was $125^{\circ} \mathrm{C}$ and the injector temperature was $230^{\circ} \mathrm{C}$. Viable cell 
number was determined by spread plate count. Furfural, HMF, vanillin and vanillic acid concentrations were determined by HPLC (Waters Model 996, Milford, MA) equipped with a photodiode array detector (at $254 \mathrm{~nm}$ ) and PRP X300 ion exclusion column (Hamilton) at the ambient temperature. The mobile phase used was $A=9: 1(1 \mathrm{mN}$ $\mathrm{H}_{2} \mathrm{SO}_{4}$ : acetonitrile) and $\mathrm{B}=5: 2\left(1 \mathrm{mN} \mathrm{H}_{2} \mathrm{SO}_{4}\right.$ : acetonitrile) at a flow rate of $1 \mathrm{~mL} \mathrm{~min}^{-1}$. The linear gradient was programmed $0-40 \% \mathrm{~B}$ in $0-3 \mathrm{~min}, 40-60 \% \mathrm{~B}$ in $3-23 \mathrm{~min}$ and $60-65 \%$ B in 23-33 min. Furfural, HMF, vanillin or vanillic acid standard solutions were made to serve as the reference for quantification. Sugar concentration was determined by another HPLC (Buck Scientific, East Norwalk, CT) equipped with a refractive index detector and Aminex HPX-87P column (Biored). The mobile phase was degased HPLC water at a flow rate of $0.6 \mathrm{~mL} \mathrm{~min}{ }^{-1}$ and the column temperature was $85^{\circ} \mathrm{C}$.

\section{Statistical Analysis}

Samples were run in triplicates. All results are expressed as mean \pm 1 SD. Differences between treatment groups were tested by one-way analysis of variance (ANOVA) using the SAS System for Windows (V8, SAS Institute Inc., Gary, NC). Differences at $p<0.05$ are considered significant.

\section{RESULTS AND DISCUSSION}

\section{Detoxification of Inhibitors by Ion Exchange Resins}

Since the inhibitor compounds may co-exist in acid hydrolyzates as a mixture and may act synergistically in inhibiting fermentation activity [3], an inhibitor cocktail containing $2 \mathrm{mg} \mathrm{mL}^{-1}$ each of furfural, HMF, vanillin and vanillic acid ( $\mathrm{pH} 0.63$ ) was treated with the resins. Added amount and the final $\mathrm{pH}$ value represent compromised numbers reported in the aforementioned literatures and what we detected in the study hydrolyzates. Both Dowex M43 and Monosphere 66 ion exchange resins were found to effectively neutralize inhibitor cocktail and significantly decrease the concentration of the individual furan and phenolic compounds. The $\mathrm{pH}$ of the solutions was raised from 0.63 to 3.5 and 4.0 after treatment with Dowex M43 and Monosphere 66 ion exchange resins for $20 \mathrm{~min}$, respectively. Fig. 1 shows that after $20 \mathrm{~min}$ of treatment, Dowex M43 and Monosphere 66 removed $67 \%$ and $66.5 \%$ of furfural, and $62 \%$ and $62 \%$ of $\mathrm{HMF}$, respectively. There was no statistical difference between the two resins in their capacity 
of removing the furans $(P>0.05)$. The resins were more effective in removing the phenolic compounds.than that for the furans. Both resins effectively removed more than $95 \%$ of vanillin (Figure 1) and $100 \%$ of vanillic acid (data not shown). Inhibitor concentrations in the samples were actually measured after 30 and $60 \mathrm{~min}$ of the treatment with the resins and the $\mathrm{pH}$ was stabilized at 4.5-5. Concentrations of both furans and phenolics were not significantly changed beyond $20 \mathrm{~min}(P>0.05)$.

\section{Effectiveness of Resins in Detoxification of the Inhibitor Cocktail}

To determine the effectiveness of the resins in detoxification, the inhibitorscontaining fermentation medium was inoculated with S. cerevisiae after detoxification. Results showed that the ethanol production as well as the viable cell count in the treated cocktail were not affected as compared with the control group (untreated fermentation medium, $\mathrm{pH}$ 6.7) (Figure 2), suggesting that after treatment the residual total furan and phenolic compounds in the cocktail were reduced to non-inhibitory concentrations. Therefore, the anion exchange technique detoxified the test inhibitors present in the cocktail used for this study.

\section{Sugar Recovery}

Maintaining a high sugar concentration in acid hydrolyzate is critical and beneficial to ethanol fermentation. To determine if detoxification by ion exchange would result in significant sugar loss, two sugar cocktails simulating the two acid hydrolyzates used in our study were tested with the resins. Table 1 shows that the treatment resulted in about $21-23 \%$ and $32-43 \%$ total sugar loss in sugar cocktails \#1 and \#2, respectively. We speculated the higher sugar loss in sugar cocktail \#2 was caused by the capability limit of the resins in handling ion exchange and retaining the sugars. There was no significant difference between the two resins in sugar retention capability $(P>0.05)$. Additional treatments with the resins by water-washing and filtering increased sugars retention rate to $95 \%$ (data not shown). However, concentration of the sugars in the filtrate could drop after repetitive treatments, pending on the volume of the water used for washing.

Processing method and feedstock are considered as the determining factors in ethanol production costs. Currently in the U.S., corn is the primary feedstock for 
ethanol production and accounts for $60 \%$ of the total production cost [17]. Use of sawdust, a lignocellulosic biomass of no or low commercial value, could significantly reduce ethanol production costs and provide an environmentally friendly renewable energy source. However, use of sulfuric acid for hydrolysis and the resins for treatment added significant costs to the presumable low-cost ethanol production method.

Theoretically, the resins can be washed, regenerated, and reused for unlimited repetitive treatments. Physically we managed reusing the resins for 20 times without reduction in detoxification efficiency. Purchasing the resins and sulfuric acid at bulk rate could further reduce our procurement cost by three- to five-fold (personal communication with the vendor). Based on our calculation, the actual ethanol production cost is about $\$ 0.20 \mathrm{~mL}^{-1}$ with one-step resin treatment. The theoretical cost can be lowered to $\$ 0.04 \mathrm{~mL}^{-1}$ if the resins can be reused for 100 times. Apparently this is not price-competitive compared to the current corn-based ethanol retail price $\left(\sim 0.0004 \mathrm{~mL}^{-1}\right)$ or Brazilian (sugarcane-based) ethanol retail price $\left(\sim 0.0002 \mathrm{~mL}^{-1}\right)$ [18].

Cheap energy, a major factor in the production of various goods from raw materials, has been heavily subsidized by the U.S. governments, and that hinders the public efforts in recycling natural resources and exploring renewable energy sources. In building a sustainable future, we must find strategies to better manage both renewable and nonrenewable resources. Recently, the average national retail price for regular unleaded gasoline has been skyrocketed to exceed $\$ 3.00$ per gallon in our neighborhood; a phenomenon signaling gradual depletion of fossil fuels. Therefore, the corn-based or the sugarcane-based ethanol is becoming price-competitive against gasoline nowadays. However, extensive transformation of rainforest areas by sugarcane farming is not sustainability friendly and corn-based ethanol production has driven up the corn price in the U.S. If governments can provide suitable tax breaks/incentives and/or subsidies, the waste lignocellulosic biomass-based ethanol may become cost-effective in the near future. Natural disasters, such as hurricanes (eg., Hurricane Katrina) and tornadoes, generate large amounts of debris, causing considerable disposal challenges for local public officials. The debris generated includes vegetative/green debris (e.g., trees, limbs, shrubs). The disaster-related debris removal 
strategies include landfilling, recycling, and/or burning. In the past, debris from disasters was buried or burned near the community. This option is deemed less desirable, because of the side effects of smoke and fire from open burning and potential water and soil contamination from uncontrolled burial of debris. The disposal in sanitary landfills often cannot be applied to disaster debris because of the large volume of wastes and reluctance to overburden the existing disposal capacity. Recycling or reuse of disaster debris is becoming more attractive for economy and sustainability reasons. Although currently lignocellulosic biomass-based ethanol production approach is not as price competitive as the corn-based technique; this approach could significantly reduce the debris disposal cost, generate a desirable fuel chemical, and help maintaining ecological sustainability.

To sum up the aforementioned discussion, the major disadvantage of resin treatment method is the cost-effectiveness compared to the current corn-based ethanol production method. However, it may become more appealing from now on since the proposed route significantly benefits humans in building a sustainable future.

\section{CONCLUSION}

In conclusion, our results indicate that the selected Dowex anion exchange resins, Dowex M43 or Dowex Monosphere 66, could effectively neutralize acidity of the acid hydrolyzate, significantly reduce the concentration of furan and phenolic compounds and improve fermentability of the acid hydrolyzate.

\section{ACKNOWLEDGEMENTS}

This research was supported by the U.S. Department of Energy \#DE-FG02O0ER45830 with subcontract to JSU through Mississippi State University. 


\section{REFERENCES}

1. Sun, Y.J.; Cheng, J. Hydrolysis of lignocellulosic materials for ethanol production: a review. Bioresor. Technol. 2002, 83, 1-11.

2. Palmqvist, E.; Hahn-Hägerdal, B. Fermentation of lignocellulosic hydrolysates. I: inhibition and detoxification. Bioresor. Technol. 2000, 74, 17-24.

3. Luo, C.; Brink, D.L.; Blanch, H.W. Identification of potential fermentation inhibitors in conversion of hybrid poplar hydrolyzate to ethanol. Biomass Bioenerg. 2002, 22, 125-138.

4. Martin, C.; Jonsson, L. Comparison of the resistance of industrial and laboratory strains of Saccharomyces and Zygosaccharomyces to lignocellulose-derived fermentation inhibitors. Enzyme Microb. Technol. 2002, 32, 386-395.

5. Martinez, A.; Rodriguez, M.E.; Wells, M.L.; York, S.W.; Preston, J.F.; Ingram, L.O. Detoxification of dilute acid hydrolysates of lignocellulose with lime. Biotech. Prog. 2001, 17, 287-293.

6. Sanchez, B.; Bautista, J. Effects of furfural and 5-hydroxymethylfurfural on the fermentation of Saccharomyces cerevisiae and biomass production from Candida guilliermondii. Enzyme Microb. Technol. 1988, 10, 315-318.

7. Azhar, A.F.; Bery, M.K.; Colcord, A.R.; et al. Factors affecting alcohol fermentation of wood acid hydrolyzate. Biotechnol. Bioeng. Symp. 1981, 11, 293-300.

8. Chung, I.S.; Lee, Y.Y. Ethanol fermentation of crude acid hydrolyzate of cellulose using high-level yeast inocula. Biotechnol. Bioeng. 1985, 27, 308-315.

9. Ingram, M.; Mossel, D.A.A.; de Lange, P. Factors, produced in sugar-acid browning reaction, which inhibit fermentation. Chem. Ind. Jan. 1955, 63-64.

10. Boopathy, R.; Bokang, H.; Daniels L Biotransformation of furfural and 5- hydroxymethyl furfural by enteric bacteria. J. Ind. Microbiol. 1993, 11, 147-150.

11. Delgenes, J.P.; Moletta, R.; Navarro, J.M. Effect of lignocellulose degradation products on ethanol fermentation of glucose and xylose by Saccharomyces cerevisiae, Zymomonas mobilis, Pichia stipitis, and Candida shehatae. Enzyme Microb. Technol. 1996, 19, 220225.

12. Pfeifer, P.A.; Bonn. G.; Bobleter, O. Influence of biomass degradation products on the fermentation of glucose to ethanol by Saccharomyces carlsbergensis W34. Biotechnol. Lett. 1984, 6, 541-546.

13. Ando, S.; Arai, I; Kiyoto, K. et al. Identification of aromatic monomers in steam-exploded poplar and their influences on ethanol fermentation by Saccharomyces cerevisiae. J. Ferment. Technol. 1986, 64, 567-570.

14. Springfield, R.M.; Hester, R.D. Continuous ion-exclusion chromatography system for acid/sugar separation. Sep. Sci. Technol. 1999, 34, 1217-1241.

15. Benson, H.J. Microbiologic applications. WmC. Brown communications, Inc. Dubuque, IA, 1994, pp, 79-84. 
16. Zhang, Y.; Hwang, H.; Begonia, M.F.T.; Lee, K.; Zeng, K. Effect of an acid hydrolyzate of southern pine softwood on the growth and fermentation ability of yeast Saccharomyces cerevisiae. J. Miss. Acad. Sci. 2005, 50, 138-143.

17. Kim, S.; Dale, B.E. Allocation procedure in ethanol production system from corn grain. 7 LCA 2002, 4, 237-243.

18. Severinghaus, J. Why we import Brazilian ethanol? http://www.iowafarmbureau.com/programs/commodity/information/pdf/Trade\%20M atters\%20column\%20050714\%20Brazilian\%20ethanol 2005. 


\section{FIGURES}
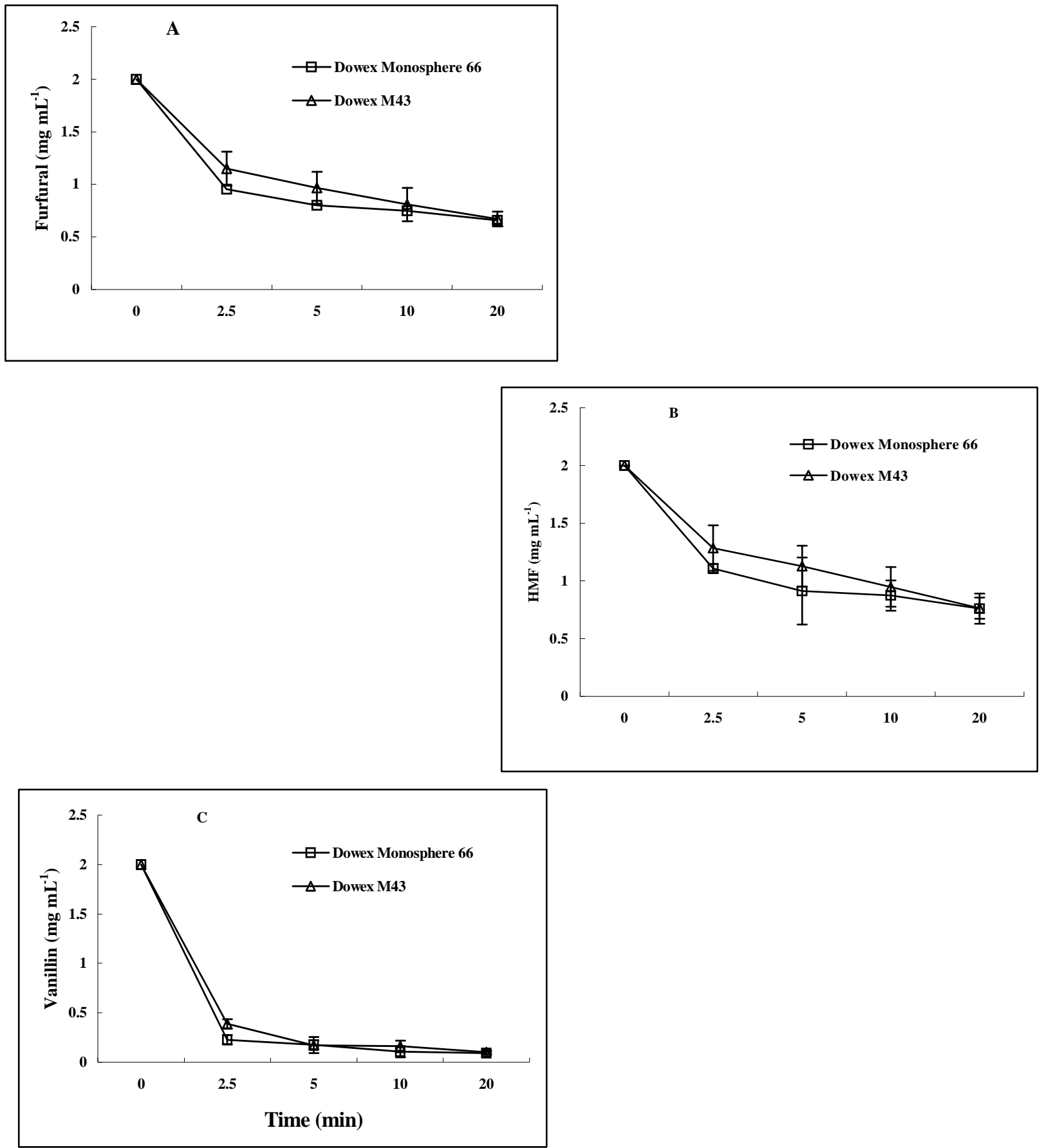

Figure 1. Detoxification of an inhibitor cocktail containing $2 \mathrm{mg} \mathrm{mL}^{-1}$ each of A) furfural, B) HMF, vanillin, and C) vanillic acid by Dowex M43 or Dowex Monosphere 66 ion exchange resins. Inhibitor solution:resin (wet weight) =1:1 (vol:wt). Samples were triplicated. Data were expressed as mean \pm 1 SD. 
A

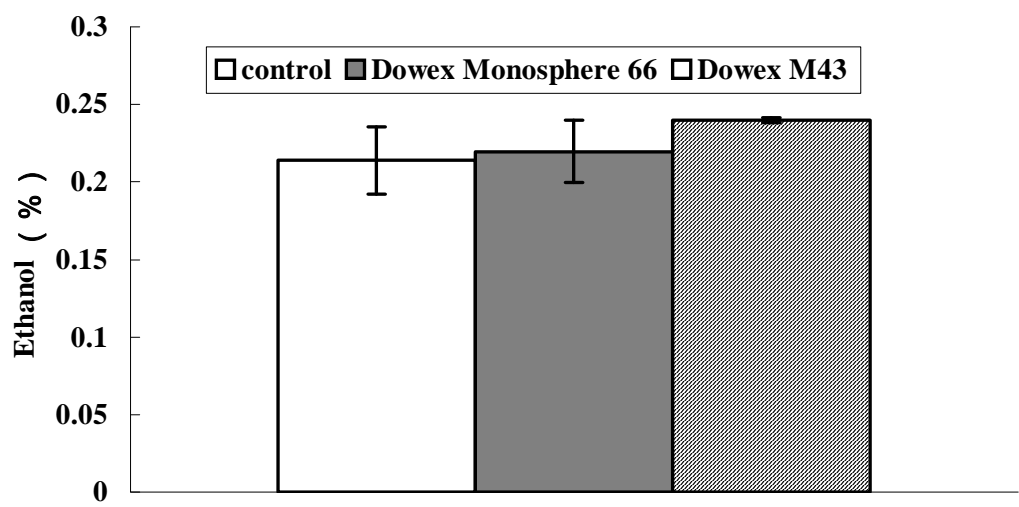

B

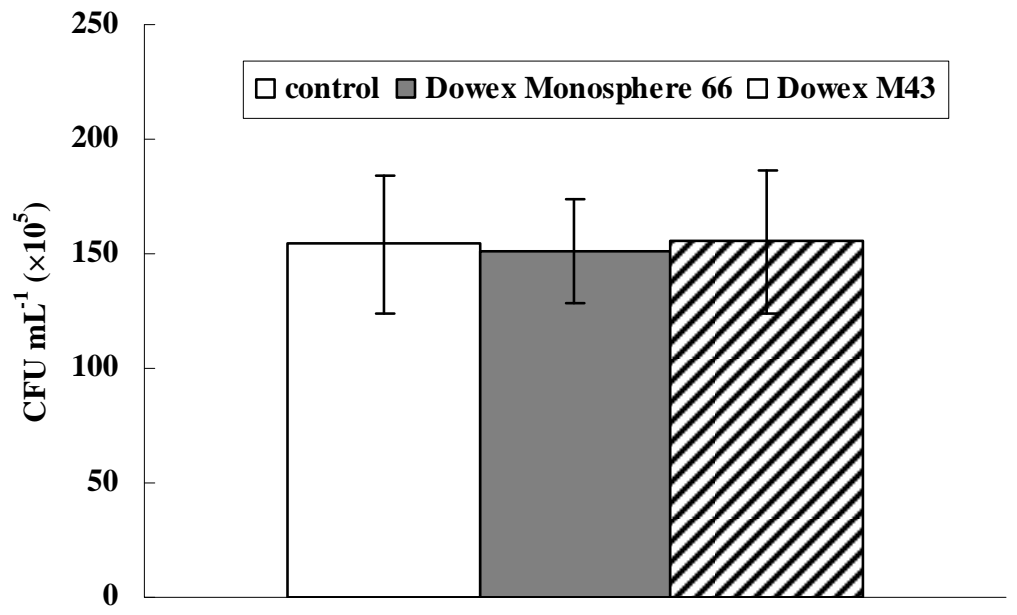

Figure 2. Effect of detoxification with ion exchange resins on A) ethanol production and B) colony forming units (cfu). The inhibitors containing fermentation medium was inoculated with S. cerevisiae after detoxification by Dowex M43 or Dowex monosphere 66 ion exchange resins. Samples were triplicated. Data were expressed as mean \pm 1 SD. 


\section{TABLE}

Table 1. Percentage of sugar recovery after treatment by Dowex M43 or Dowex Monosphere 66 ion exchange resins.

\begin{tabular}{|c|c|c|c|}
\hline \multicolumn{2}{|c|}{ Sugar $\left(\mathrm{mg} \mathrm{mL}^{-1}\right)$} & Dowex M43 (\%) & Dowex Monosphere 66 (\%) \\
\hline \multicolumn{4}{|c|}{ Cocktail \#1 } \\
\hline Glucose & 2.5 & $69.9 \pm 0.1$ & $69.0 \pm 1.1$ \\
\hline Xylose & 1.0 & $72.8 \pm 5.6$ & $75.5 \pm 3.0$ \\
\hline Galactose & 1.5 & $93.6 \pm 16.3$ & $100.0 \pm 7.1$ \\
\hline Mannose & 4.0 & $76.7 \pm 6.1$ & $80.0 \pm 4.4$ \\
\hline Total & 9.0 & $77.3 \pm 2.2$ & $78.7 \pm 1.7$ \\
\hline \multicolumn{4}{|c|}{ Cocktail \#2 } \\
\hline Glucose & 15.0 & $59.8 \pm 0.6$ & $66.5 \pm 10.4$ \\
\hline Xylose & 3.0 & $57.8 \pm 3.6$ & $70.7 \pm 14.6$ \\
\hline Galactose & 2.0 & $55.7 \pm 4.3$ & $72.7 \pm 15.1$ \\
\hline Arabinose & 1.0 & $49.4 \pm 8.7$ & $64.7 \pm 13.8$ \\
\hline Mannose & 5.0 & $61.0 \pm 1.3$ & $74.1 \pm 13.1$ \\
\hline Total & 26.0 & $59.0 \pm 1.0$ & $68.3 \pm 11.4$ \\
\hline
\end{tabular}




\section{MEMBRANE SEPARATIONS PROCESSES IN THE CONVERSION OF BIOMASS TO FUELS \\ Performer: University of Southern Mississippi}

\section{INTRODUCTION}

This phase of the effort focused on membrane separation processes developed for two critical components of the conversion of cellulosic biomass to fuel ethanol. Membrane separation of the acid-sugar mixture produced in concentrated acid hydrolysis was accomplished using an electrodialysis process, and separation of the ethanol-water mixture produced in fermentation was accomplished using a hybrid distillation-pervaporation process and/or perstraction techniques in conjunction with the fermentation reactors. Traditional distillation concentrated the mixture to the azeotrope, - 95\% ethanol/5\% water, with membrane pervaporation applied to further dehydrate to $>99 \%$ pure ethanol (Fuel grade).

Previous research at the University of Southern Mississippi focused on developing an understanding of ionomer materials and the specific impact of ionic functionalities on polymer properties [1]. The current research will use this foundation in ionomer morphology to tailor membrane morphology to achieve more efficient separations. To date work has focused on the production of potential membrane materials, most promising being sulfonated ethylene-vinyl alcohol copolymers. These polymers show classic ionomer behaviors, ionic aggregation and resultant microphase separation, as examined by Na NMR and SAXS. Differential scanning calorimetry has shown a $50^{\circ} \mathrm{C}$ increase in glass transition temperature over the parent copolymer indicating a significant impact on chain mobility with sulfonation [2].

\section{MEMBRANE PROCESSES FOR ACID-SUGAR SEPARATION OF A HYDROLYZATE FEED}

Published reports on acid-sugar separations have shown effective separation using ion exclusion chromatography (IEC) and electrodialysis. Using a pilot scale IEC system, Springfield showed effective separation using commercially available resins [3]; however, it has been realized that membrane processes offer a greater economic benefit. For example, Goldstein and coworkers demonstrated the use of electrodialysis with conventional ion-exchange membranes, an electrically driven membrane 
separation process, to separate a model hydrolyzate yielding a glucose isolation costing about $\$ 0.02$ per lb. [4]. For our next phase of research in this project, we will define this electrodialysis process as the state-of-the-art technological benchmark against which our future developments will be compared.

Research efforts in acid-sugar separations will focus on the development of novel cation exchange membranes, specifically sulfonated ethylene-vinyl alcohol copolymers. Membrane performance will be evaluated as a function of crosslink density [5], sulfonation level [6] and membrane preparation technique. Electrodialysis of a model hydrolyzate will be studied using the developed cation exchange membrane and a commercially available anion exchange membrane. Future developments will focus on enhanced anion exchange membranes. Using a commercially available electrodialysis unit, real-time separation efficiency will be monitored using standard $\mathrm{pH}$ measurements and Near-IR spectroscopy [7]. The mechanical integrity and lifetime evaluations of the membranes subjected to the harsh acid environments will be studied using tensile testing and dynamic mechanical analysis (DMA).

\section{MEMBRANE PROCESSES FOR ETHANOL ISOLATION AND DEHYDRATION}

\section{Ethanol Isolation}

Although many microorganisms are known to be useful in the fermentation process, when the fermentation product concentration reaches a maximum value the organisms lose their effectiveness and the fermentation rate declines to zero. The production period of the microorganisms may be extended by continuous extraction of ethanol from the fermentation reactor in order to reduce concentrations of the fermentation products. In doing so, the nutrients and other agents necessary for fermentation are conserved, the production capacity of the fermentation equipment is raised, and the overall profitability of the fermentation operation can be increased.

During this next research period, the separations group at MSU (headed by the George research group) will set up and operate a bench-scale fermentation system. The objective of the project will be to use membrane separation techniques to remove fermentation products as they are produced in the reactor, thus decreasing the inhibition of the microorganism. The membrane separation technique to be investigated is liquid 
membrane perstraction. Liquid membrane perstraction utilizes a membrane that is exposed to the fermentation reactor liquor on one side and a sweep solvent on the other side. The nature of the membrane is such that the ethanol or other fermentation product travels from the fermentation liquor through the membrane and into a sweep solvent. The fermentation products are then separated from the sweep solvent by other techniques such as distillation.

\section{Ethanol Dehydration}

While conventional distillation processes are effective in yielding concentrated ethanol streams (up to the ethanol-water azeotrope of ca. 95\% ethanol), fuel grade ethanol (e.g., for mixtures with gasoline) must be anhydrous. Membrane processes involving pervaporation have been demonstrated to be economically viable methods to reach these high ethanol concentrations (> 99\%) in an environmentally friendly manner. While literature reveals a multitude of potential membrane materials, only crosslinked poly (vinyl alcohol) membranes have been used extensively on the commercial scale. As a result of this literature study, the PERVAP ${ }^{\circledR}$ series of poly (vinyl alcohol) membranes from Sulzer Chemtech will be used as the performance benchmark for this on-going investigation.

Based on our recent findings of extraordinary differential swelling characteristics in ethanol and water, our novel sulfonated ethylene-vinyl alcohol materials will be explored in pervaporation applications. As above, the effect of varying crosslink density, sulfonation and membrane preparation techniques on pervaporation performance will be evaluated. Separations will be conducted on a $95 \%$ ethanol $/ 5 \%$ water mixture using an in-house constructed pervaporation system. Separation efficiency will be evaluated using both selectivity and flux; selectivity being determined though gas chromatography and flux through gravimetric techniques [8]. 


\section{REFERENCES}

1. Orler, E. B.; Calhoun, B. H.; and Moore, R. B. Macromolecules 1996, 29, 59655971.

2. Pan, B., and Moore, R.B., Unpublished results 2002.

3. Springfield, R. M. In Department of Polymer Science; University of Southern Mississippi: Hattiesburg, 1999; p 286.

4. Goldstein, I. S.; Bayat-Makooi, F.; Sabharwal, H. S.; and Singh, T. M. Applied Biochemistry and Biotechnology 1989, 20/21, 95-106.

5. Sheilbey, D. W.; Manzo, M. A.; and Gonzalez-Sanabria, O. D. J. Electrochemical Society 1983, 130, 255-259.

6. Goethals, E. J., and Natus, G., Die Makromolekulare Chemie 1968, 116, 152-157.

7. Chung, H.; Arnold, M. A.; Rhiel, M., and Murhammer, D. W. Applied Biochemistry and Biotechnology 1995, 50, 109-125.

8. Huang, R. Y. M., and Yeom, C. K. J. Membrane Science 1990, 51, 273-292. 